\title{
PROPERTIES AND APPLICATIONS OF THE AVERAGE INTERPARTICLE DISTAMCE.
}

JOSHUA W. HOLETT 


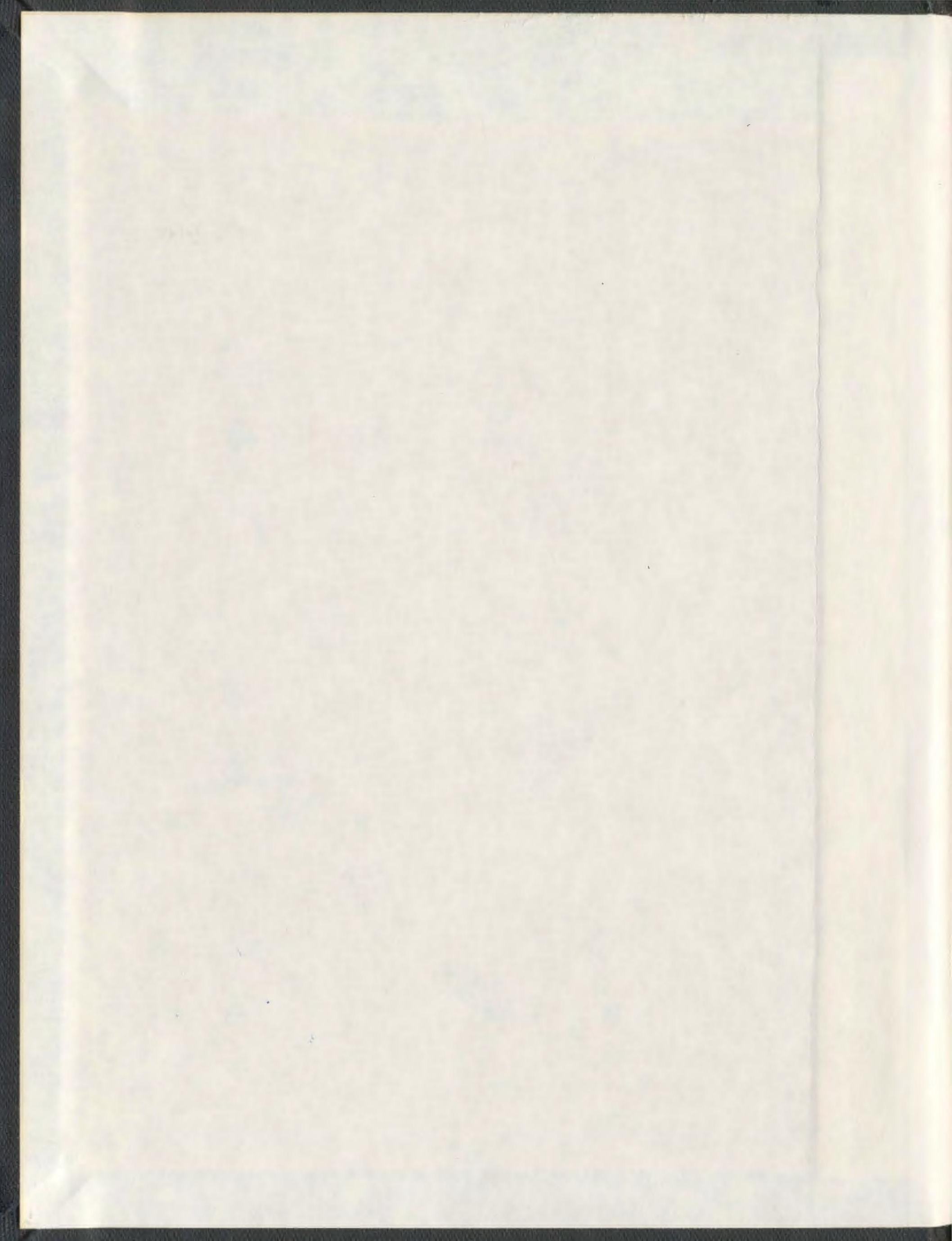


001311

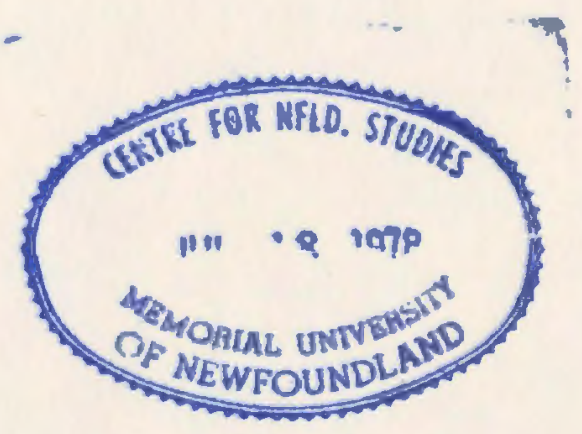





\title{
Properties and Applications of the Average Interparticle Distance
}

\author{
by \\ (c) Joshua W. Hollett

\begin{abstract}
A thesis submitted to the
School of Graduate Studies

in partial fulfilment of the

Doctor of Philosophy
\end{abstract} \\ requirements for the degree of \\ Department of Chemistry \\ Memorial University of Newfoundland
}

May 2009

St. John's

Newfoundland 


\begin{abstract}
The first and second moment operators are used to define the origin invariant shape and size of a molecule or functional group, as well as expressions for the distance between two electrons and the distance between an electron and a nucleus. The measure of molecular size correlates quite well with an existing theoretical measure of molecular volume calculated from isodensity contours. Also, the measure of size is effective in predicting steric effects of substituents which have been measured experimentally. The electron-electron and electron-nuclear distances are related to components of the Hartree-Fock energy. The average distance between two electrons models the Coulomb energy quite well, especially in the case of localized molecular orbitals. The average distance between an electron and a nucleus is closely related to the electron-nuclear attraction energy of a molecule.

The relationships discovered between the average interparticle distances and molecular energy components have led to the development of a new empirical approach to modelling the electronic structure of molecules. The general energy expression for a simulated electronic structure theory is defined, along with the functional form of the interatomic distance dependent energy functions. The theory is used to model the hydrogen molecule, the first-row hydrides, and ethane. The models, which have the correct RHF/6-31G(d) optimized geometries, also fit the RHF/6-31G(d) energy at equilibrium and the UHF/6-31G(d) energy at the bond dissociation limit, as well as some vibrational frequencies.
\end{abstract}

Also directly related to the interelectronic distance, is the issue of electron correlation. Several new approaches to the electron correlation problem have emerged 
in recent years. Among the new methods is orbital functional theory, in which the correlation energy is a functional of the molecular orbitals. The correlation energy of different isoelectronic series as a function of nuclear charge is investigated in an effort to design a correlation operator. Insight is also gained by examining the explicit CISD energy expression. 


\section{Acknowledgements}

I would like to thank:

Dr. R. A. Poirier, not just the best supervisor a student could have, but a great role model. Who knows what I would be doing if our paths had not crossed;

my parents, Harvey and Barbara, who always believed I can do anything, but will support me in whatever I do;

my wife, Natasha, for love and support, and helping me be myself. I could not have done it without you. 


\section{Contents}

Abstract

Acknowledgements $\quad$ iv

List of Tables $\quad$ ix

List of Figures $\quad$ x

1 Introduction 1

1 Hartree-Fock Theory ..................... 3

2 Electron Correlation ..................... 9

3 Post Hartree-Fock Methods . . . . . . . . . . . . . . . . . 10

4 Density Functional Theory . . . . . . . . . . . . 15

5 Molecular Mechanics ................. 20 20

References .............................. 24

2 Quantum mechanical size and steric hindrance $\quad 27$

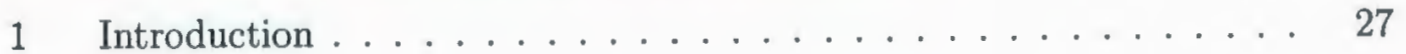

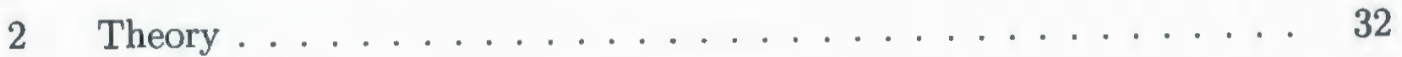


2.1 The origin invariant electronic second moment tensor of a molecule 32

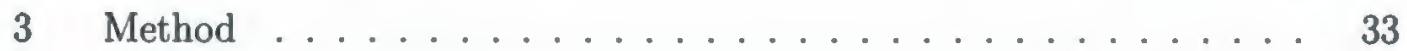

4 Results and Discussion ................... 34

4.1 Molecular Shape and Volume ............ 34

$4.2 \quad$ Steric Effects . . . . . . . . . . . . . . . 39

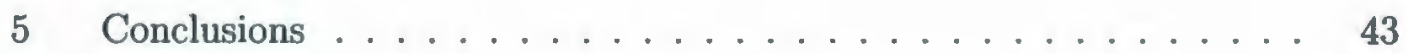

References ............................. 44

3 An interesting relationship between interelectronic distance and the $\begin{array}{ll}\text { corresponding Coulomb integral } & 47\end{array}$

1 Introduction ........................ 47

2 Theory ................................ 50

2.1 The Distance Between Two Electrons, $\left(\delta r_{12}\right)_{a b} \ldots \ldots 50$

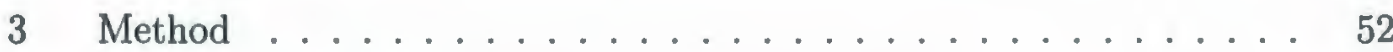

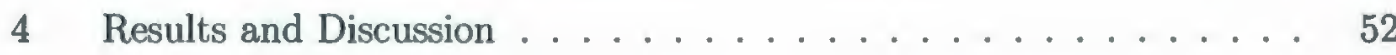

4.1 Behaviour of $\left(\delta r_{12}\right)_{a b} \ldots \ldots \ldots . \ldots \ldots 2$

4.2 Relationship with the two-electron Coulomb integral, $J_{a b} \ldots \quad 55$

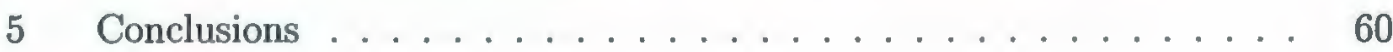

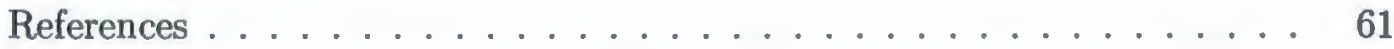

4 Properties and applications of the average interparticle distance $\quad 64$

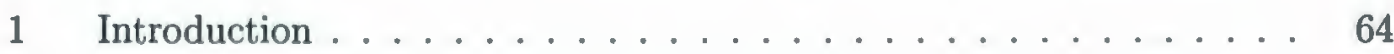

2 Theoretical definition of the shape and size of a molecule or functional

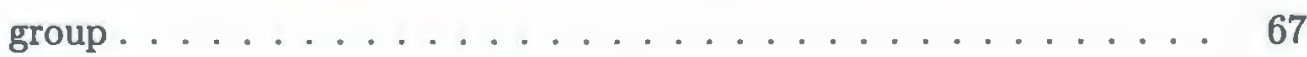

3 The average interparticle distance .............. 73 
3.1 The average interelectronic distance ......... 73

3.2 $\left(\delta r_{12}\right)_{a b}$ and the corresponding Coulomb and exchange integrals 75

3.3 The average electron-nucleus distance and the electron-nucleus potential energy . . . . . . . . . . . . . . 80

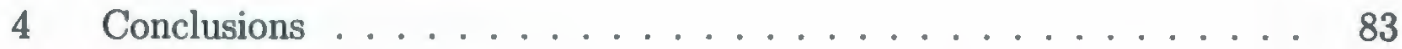

5 Acknowledgements ....................... 84

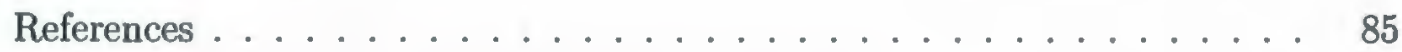

5 SEST: Simulated Electronic Structure Theory 88

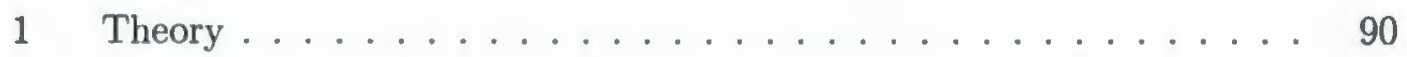

1.1 Simulated Electronic Structure Theory (SEST) ...... 90

1.2 The average interparticle distance and modelling molecular orbital energy components ................. 93

1.3 Functional Form ................. 100

1.4 Determining the Parameters .............. 103

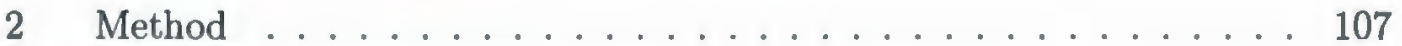

3 Results and Discussion .................... 108

3.1 Hydrogen molecule and the first-row hydrides . . . . . 108

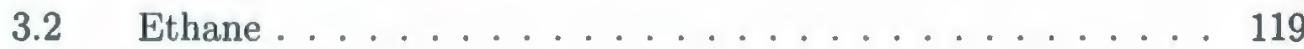

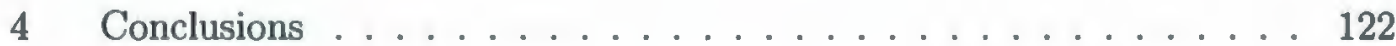

5 Acknowledgements ....................... 123

References ............................. 124

6 Development of a new electron correlation theory 127

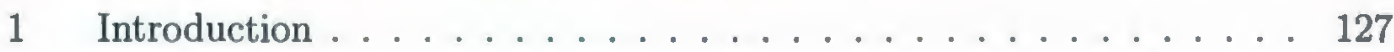


1.1 Electron correlation . . . . . . . . . . . . 127

1.2 Explicitly correlated wave functions and exact energies of atomic

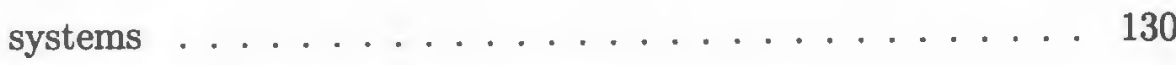

1.3 Traditional wave function methods . . . . . . . . . . 134

1.4 Density Functional Theory . . . . . . . . . . . . . 137

$1.5 \quad$ New approaches . . . . . . . . . . . . . 142

2 A molecular orbital correlation functional . . . . . . . . . . 146

3 The explicit CISD energy expression ................ 158

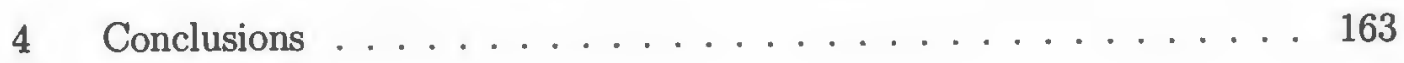

5 Acknowledgements .......................... 163

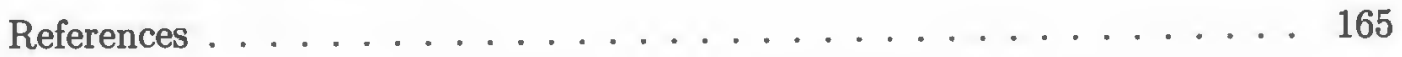

$\begin{array}{llr}7 & \text { Summary } & 170\end{array}$

$\begin{array}{lc}\text { A Density Functionals } & 173\end{array}$

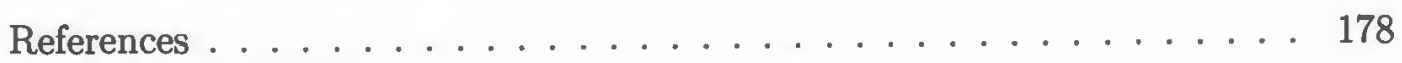




\section{List of Tables}

2.1 Molecular shape and volume from origin invariant electronic second moment $(\mathrm{HF} / 6-31 \mathrm{G}(\mathrm{d}) / / \mathrm{HF} / 6-31 \mathrm{G}(\mathrm{d})) \ldots \ldots 38$

5.1 Derivation of SEST electron-nucleus potential energy parameters for ammonia from RHF/6-31G(d) LMO energy components. ${ }^{a}$. . . . . 105

5.2 Derivation of SEST electron-electron potential energy parameters for ammonia from UHF/6-31G(d) LMO energy components. ${ }^{a}$. . . . . 106

5.3 SEST and RHF/6-31G(d) vibrational frequencies for the first-row hydrides ................................. 117 


\section{List of Figures}

2.1 Topomerization of phane system with intra-annular substituent, $X$, used to determine $n$-values. ${ }^{7} \ldots \ldots \ldots 29$

2.2 Comparison of average radii of hydrides to van der Waals and BraggSlater radii ......................... 34

2.3 Comparison of electronic second moment average radius, $\widetilde{R}$, to Bader isodensity contour molecular volume, ${ }^{12} V_{\rho}(\rho=0.002$ a.u. $) . \ldots 36$

2.4 Relationship between the electronic second moment average radius, $\widetilde{R}$, and the modified Taft steric constant, $E_{S}^{e}{ }^{3} \ldots \ldots \ldots 39$

2.5 Relationship between the electronic second moment average radius, $\widetilde{R}$,

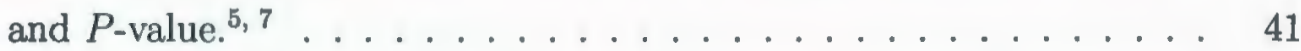

2.6 Relationship between the electronic second moment average radius, $\widetilde{R}$,

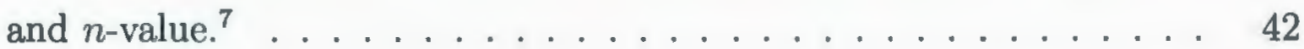

$3.1\left(\delta r_{12}\right)_{a b}$ of two-electron singlet systems with varying nuclear charge. . $\quad 53$

$3.2\left(\delta r_{12}\right)_{a b}$ of two-electron triplet systems with varying nuclear charge. . $\quad 54$

3.3 Relationship between $\left(\delta r_{12}\right)_{a a}$ and Coulomb integral, $J_{a a}$, for the singlet state of $\mathrm{He}$ to $\mathrm{Ne}^{8+} \ldots \ldots \ldots \ldots \ldots$ 
3.4 Relationship between $\left(\delta r_{12}\right)_{a b}$ and Coulomb integral, $J_{a b}$, for the triplet state of $\mathrm{He}$ to $\mathrm{Ne}^{8+} \ldots \ldots \ldots \ldots \ldots \ldots$

3.5 Relationship between $\left(\delta r_{12}\right)_{a b}$ and corresponding Coulomb integral, $J_{a b}$, for systems of four and ten electrons, calculated at HF/6-31G(d) using CMOs and LMOs. .......................... 58

3.6 Relationship between $\left(\delta r_{12}\right)_{a b}$ and corresponding Coulomb integral, $J_{a b}$, for all systems, calculated at $\mathrm{HF} / 6-31 \mathrm{G}(\mathrm{d}) \ldots \ldots \ldots \ldots$

4.1 The shape and size of some small molecules $\left(\mathrm{H}_{2}, \mathrm{H}_{2} \mathrm{O}, \mathrm{CH}_{3} \mathrm{OH}\right.$, and $\mathrm{CHOOH}$ ) calculated at $\mathrm{HF} / 6-31 \mathrm{G}(\mathrm{d})$. Molecules are slightly rotated off the indicated principal axes to show all atoms. . . . . . . . .

4.2 Comparison of electronic second moment average radius, $\widetilde{R}$ (HF/6$31 \mathrm{G}(\mathrm{d}))$, to Bader isodensity contour molecular volume, ${ }^{13} V_{\rho}(\rho=0.002$

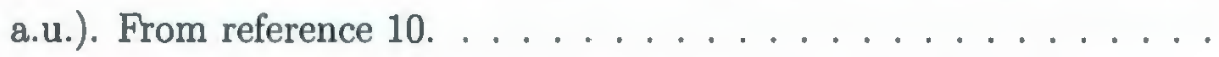

$4.3\left(\delta r_{12}\right)_{a b}$ of two-electron singlet and triplet systems with varying nuclear charge $\left(\mathrm{He}\right.$ to $\left.\mathrm{Mg}^{10+}\right)$. Note that some points overlap. . . . . . . . . .

4.4 Relationship between Coulomb energy, $J_{a b}$, and the average distance between two electrons, $\left(\delta r_{12}\right)_{a b}$, for two-electron ions to molecules with up to 58 electrons $(\mathrm{HF} / 6-31 \mathrm{G}(\mathrm{d}), \mathrm{CMO}) \ldots \ldots \ldots \ldots$

4.5 Relationship between Coulomb energy, $J_{a b}$, and the average distance between two electrons, $\left(\delta r_{12}\right)_{a b}$, for two-electron ions to molecules with up to 58 electrons $(\mathrm{HF} / 6-31 \mathrm{G}(\mathrm{d}), \mathrm{LMO}) \ldots \ldots \ldots$ 
4.6 Relationship between exchange energy, $K_{a b}$, and the average distance between two electrons, $\left(\delta r_{12}\right)_{a b}$, for three and four-electron systems with varying nuclear charge $(\mathrm{ROHF} / 6-31 \mathrm{G}(\mathrm{d})) \ldots \ldots \ldots$

4.7 Relationship between electron-nucleus potential energy, $V_{a a}$, and the average distance between an electron and a nucleus, $\delta r_{a A}$, for twoelectron ions to molecules with up to 58 electrons (HF/6-31G(d), CMO). 81

4.8 Relationship between electron-nucleus potential energy, $V_{a a}$, and the average distance between an electron and a nucleus, $\delta r_{a A}$, for twoelectron ions to molecules with up to 58 electrons (HF/6-31G(d), LMO). 82

5.1 Relationship between Coulomb energy, $J_{a b}$, and the average distance between two electrons, $\left(\delta r_{12}\right)_{a b}$, for two electron ions to molecules with up to 58 electrons $(\mathrm{HF} / 6-31 \mathrm{G}(\mathrm{d}), \mathrm{LMO})$. From reference $7 . . .$.

5.2 Relationship between electron-nuclear attraction energy, $V_{a A}$, and the average distance between an electron and a nucleus, $\delta r_{a A}$, for two electron ions to molecules with up to 58 electrons (HF/6-31G(d), LMO).

5.3 Relationship between the average distance between two electrons, $\left(\delta r_{12}\right)_{a b}$, and the fluorine-fluorine distance, $R_{\mathrm{FF}}$, in $\mathrm{F}_{2}$ (HF/6-31G(d), LMO). Includes $a=b=$ bond, $a=$ bond $b=$ core, $a=$ core $b=$ core, and $a=$ lone pair $b=$ lone pair(from separate nuclei). . . . . . . . 98

5.4 Relationship between the average distance an electron and a nucleus, $\delta r_{a A}$, and the fluorine-fluorine distance, $R_{\mathrm{FF}}$, in $\mathrm{F}_{2}(\mathrm{HF} / 6-31 \mathrm{G}(\mathrm{d})$, LMO). Includes $a=$ bond, core, and lone pair. . . . . . . . . 99

5.5 Dissociation curve for $\mathrm{H}_{2}$ (SEST and RHF/6-31G(d)). . . . . 108 
5.6 Dissociation curve for $\mathrm{BeH}$ bond of $\mathrm{BeH}_{2}$ (SEST and RHF/6-31G(d)). 110

5.7 Dissociation curve for $\mathrm{BH}$ bond of $\mathrm{BH}_{3}$ (SEST and RHF/6-31G(d)). . 111

5.8 Dissociation curve for $\mathrm{CH}$ bond of $\mathrm{CH}_{4}$ (SEST and RHF/6-31G(d)). . 112

5.9 Dissociation curve for $\mathrm{NH}$ bond of $\mathrm{NH}_{3}$ (SEST and RHF/6-31G(d)). . 113

5.10 Dissociation curve for $\mathrm{OH}$ bond of $\mathrm{H}_{2} \mathrm{O}$ (SEST and RHF/6-31G(d)). . 114

5.11 Dissociation curve for HF (SEST and RHF/6-31G(d)) . . . . . 115

5.12 Dissociation curve for CC bond of ethane (SEST and RHF/6-31G(d). 119

5.13 Rigid rotation about CC bond of ethane (SEST and RHF/6-31G(d)). 121

6.1 Correlation energy as a function of nuclear charge, $Z$, for isoelectronic series of 2 to 18 electrons $(N=2-12) \ldots \ldots \ldots \ldots \ldots \ldots$

6.2 Error in energies of two electron helium-like ions, $Z=2-28$. (Other than B3LYP/6-31G(d) data, all calculations performed with a scaled aug-cc-pVQZ helium basis set.) $\ldots \ldots \ldots \ldots \ldots \ldots$

6.3 Exact correlation energy of two-electron singlet systems compared to exchange energy of two-electron triplet systems, over a range of nuclear charge, $Z=2-28$. Exchange energy calculated at UHF using a scaled aug-cc-pVQZ helium basis set. . . . . . . . . . . . . . 154

6.4 HOMO-LUMO gap of two and four-electron systems as a function of nuclear charge, $Z .(H F / 6-31 G(d)) \ldots \ldots \ldots \ldots \ldots$

6.5 Function of HOMO-LUMO energies for two and four-electron systems as a function of nuclear charge, $Z$. $(\mathrm{HF} / 6-31 \mathrm{G}(\mathrm{d})) \ldots \ldots \ldots$ 


\section{Chapter 1}

\section{Introduction}

Today, computational chemistry is used to study molecular structure and properties, and chemical kinetics and dynamics in such areas as molecular biology, protein engineering, ${ }^{1}$ surface chemistry and nanotechnology. Computational methods are becoming an increasingly important tool for explaining and predicting experimental results as well as designing and discovering new chemistry. Due to advancements of computer technology and the theory behind computational methods, computational chemistry calculations can be performed on increasingly larger systems with more accuracy. Development of $a b$ initio theories and the algorithms used for their implementation ${ }^{2}$ has allowed for the determination of highly accurate molecular wave functions. Furthermore, increased computational power along with lower level $a b$ initio, semi-empirical, or empirical methods has enabled the study of the structure and dynamics of large systems such as interacting proteins. The theory behind computational chemistry methods is vast and ranges from $a b$ initio electronic structure theory derived directly from quantum mechanics to entirely empirical, classically based, molecular mechanics. 
Each end of the spectrum has its own advantages and disadvantages.

Wave function electronic structure theory involves finding solutions to the electronic Schrödinger equation. The electronic Schrödinger equation is derived by applying the Born-Oppenheimer approximation ${ }^{3}$ to the time-independent Schrödinger equation for a molecular system (Equation 1.1).

$$
\widehat{H} \Psi\left(\left\{\mathbf{r}_{i}\right\},\left\{\mathbf{R}_{A}\right\}\right)=E \Psi\left(\left\{\mathbf{r}_{i}\right\},\left\{\mathbf{R}_{A}\right\}\right)
$$

where $\Psi\left(\left\{\mathbf{r}_{i}\right\},\left\{\mathbf{R}_{A}\right\}\right)$ is the molecular wave function, which is a function of the electronic, $\left\{\mathbf{r}_{i}\right\}$, and nuclear, $\left\{\mathbf{R}_{A}\right\}$, coordinates, and $E$ is the total non-relativistic energy of the system. The total non-relativistic Hamiltonian, $\hat{H}$, is given by

$$
\begin{aligned}
\widehat{H}= & -\sum_{i=1}^{N} \frac{1}{2} \nabla_{i}^{2}-\sum_{i=1}^{N} \sum_{A=1}^{M} \frac{Z_{A}}{r_{i A}}+\sum_{i=1}^{N} \sum_{j<i} \frac{1}{r_{i j}} \\
& +\sum_{A=1}^{M} \sum_{B<A} \frac{Z_{A} Z_{B}}{R_{A B}}-\sum_{A=1}^{M} \frac{1}{2 M_{A}} \nabla_{A}^{2}
\end{aligned}
$$

where $N$ is the total number of electrons, and $M$ is the total number of nuclei. The energy may also be expressed as an expectation value of the wave function,

$$
E=\frac{\langle\Psi|\widehat{H}| \Psi\rangle}{\langle\Psi \mid \Psi\rangle}
$$

The Born-Oppenheimer approximation states that, due to relative masses of the electrons and nuclei, the molecular wave function may be approximated by the product of the electronic and nuclear wave functions, $\Psi\left(\left\{\mathbf{r}_{i}\right\},\left\{\mathbf{R}_{A}\right\}\right)=\Psi_{\text {elec }}\left(\left\{\mathbf{r}_{i}\right\}\right) \Psi_{\text {Nuc }}\left(\left\{\mathbf{R}_{A}\right\}\right)$. Physically speaking, the electrons are said to be moving in a field of fixed nuclei. The electronic Schrödinger equation is written

$$
\widehat{H}_{\text {elec }} \Psi_{\text {elec }}\left(\left\{\mathbf{r}_{i}\right\}\right)=E_{\text {elec }} \Psi_{\text {elec }}\left(\left\{\mathbf{r}_{i}\right\}\right)
$$


where,

$$
\widehat{H}_{\text {elec }}=-\sum_{i=1}^{N} \frac{1}{2} \nabla_{i}^{2}-\sum_{i=1}^{N} \sum_{A=1}^{M} \frac{Z_{A}}{r_{i A}}+\sum_{i=1}^{N} \sum_{j<i} \frac{1}{r_{i j}}
$$

is the electronic Hamiltonian. The total energy of a given nuclear configuration is the sum of the electronic energy, $E_{\text {elec}}$, and the nuclear repulsion energy, $E_{\mathrm{NN}}$ (Equation 1.6).

$$
E_{\mathrm{NN}}=\sum_{A=1}^{M} \sum_{B<A} \frac{Z_{A} Z_{B}}{R_{A B}}
$$

The kinetic energy of the fixed nuclei is zero. The wave function, and hence the total energy, depend parametrically on the nuclear coordinates. The energy as a function of the nuclear coordinates is often referred to as the Born-Oppenheimer, or potential energy, surface. A significant part of computational chemistry is dedicated to the exploration of potential energy surfaces (PESs) of various molecular systems. While many of these investigations involve solving the electronic Schrödinger equation, there are several theories which accomplish this task to varying degrees.

\section{Hartree-Fock Theory}

The basis of most wave function electronic structure theories is the Hartree-Fock approximation. Hartree-Fock theory involves approximating the wave function by the simplest function that satisfies the Pauli antisymmetry principle, a Slater determinant (Equation 1.7). A wave function is antisymmetric if the interchange of the coordinates of any two electrons results in the change in sign of the wave function, $\Psi\left(\mathbf{x}_{1}, \mathbf{x}_{2}\right)=$ 
$-\Psi\left(x_{2}, x_{1}\right)$.

$$
\Psi\left(\mathbf{x}_{1}, \mathbf{x}_{2}, \ldots, \mathbf{x}_{N}\right)=\frac{1}{\sqrt{N !}}\left|\begin{array}{cccc}
\chi_{1}\left(\mathbf{x}_{1}\right) & \chi_{2}\left(\mathbf{x}_{1}\right) & \ldots & \chi_{N}\left(\mathbf{x}_{1}\right) \\
\chi_{1}\left(\mathbf{x}_{2}\right) & \chi_{2}\left(\mathbf{x}_{2}\right) & \ldots & \chi_{N}\left(\mathbf{x}_{2}\right) \\
\vdots & \vdots & & \vdots \\
\chi_{1}\left(\mathbf{x}_{N}\right) & \chi_{2}\left(\mathbf{x}_{N}\right) & \ldots & \chi_{N}\left(\mathbf{x}_{N}\right)
\end{array}\right|
$$

where the $\chi_{i}$ are spin orbitals and the $\mathbf{x}_{i}$ are the spatial and spin coordinates of each electron, $\mathbf{x}_{i}=\left(\mathbf{r}_{i}, \omega_{i}\right)$. The spin orbitals are products of spatial orbitals, $\phi(\mathbf{r})$, and spin functions, $\alpha(\omega)$ or $\beta(\omega)$.

$$
\chi(\mathbf{x})=\left\{\begin{array}{c}
\phi(\mathbf{r}) \alpha(\omega) \\
\text { or } \\
\phi(\mathbf{r}) \beta(\omega)
\end{array}\right.
$$

As a result of satisfying the antisymmetry principle, the electrons of the wave function are also indistinguishable, all possible permutations of electrons in spin orbitals are included.

The Hartree-Fock energy is the expectation value of the electronic Hamiltonian, $\widehat{H}$ (elec subscript is subsequently dropped), and the Hartree-Fock wave function, $E_{\mathrm{HF}}=\left\langle\Psi_{\mathrm{HF}}|\widehat{H}| \Psi_{\mathrm{HF}}\right\rangle$.

$$
E_{\mathrm{HF}}=\sum_{i=1}^{N}\left\langle\chi_{i}|\hat{h}| \chi_{i}\right\rangle+\frac{1}{2} \sum_{i=1}^{N} \sum_{j=1}^{N}\left\langle\chi_{i} \chi_{j}|| \chi_{i} \chi_{j}\right\rangle
$$

The operator $\hat{h}$ is the one-electron part of the Hamiltonian,

$$
\hat{h}=-\frac{1}{2} \nabla_{1}^{2}-\sum_{A=1}^{M} \frac{Z_{A}}{r_{1 A}}
$$

and $\left\langle\chi_{i} \chi_{j} \| \chi_{i} \chi_{j}\right\rangle$ is the antisymmetrized two-electron integral,

$$
\left\langle\chi_{i} \chi_{j}|| \chi_{i} \chi_{j}\right\rangle=\left\langle\chi_{i} \chi_{j} \mid \chi_{i} \chi_{j}\right\rangle-\left\langle\chi_{i} \chi_{j} \mid \chi_{j} \chi_{i}\right\rangle
$$


where

$$
\left\langle\chi_{i} \chi_{j} \mid \chi_{i} \chi_{j}\right\rangle=\iint \chi_{i}^{*}\left(\mathbf{x}_{1}\right) \chi_{j}^{*}\left(\mathbf{x}_{2}\right) \frac{1}{r_{12}} \chi_{i}\left(\mathbf{x}_{1}\right) \chi_{j}\left(\mathbf{x}_{2}\right) d \mathbf{x}_{1} d \mathbf{x}_{2}
$$

The Hartree-Fock equations are derived by minimizing the energy (Equation 1.9) with respect to the spin orbitals under the constraint that the spin orbitals remain orthonormal $\left(\left\langle\chi_{i} \mid \chi_{j}\right\rangle=\delta_{i j}, \forall i, j\right)$. An elegant derivation is possible using Lagrange's method of undetermined multipliers. ${ }^{4}$ The general result is also referred to as orbital Euler-Lagrange (OEL) equations. ${ }^{5}$ In the Hartree-Fock case, the equations are given by

$$
\hat{f} \chi_{i}\left(\mathbf{x}_{1}\right)=\epsilon_{i} \chi_{i}\left(\mathbf{x}_{1}\right)
$$

where $\epsilon_{i}$ is the orbital energy and $\hat{f}$ is the Fock operator.

$$
\hat{f}=\hat{h}+\sum_{j=1}^{N} \hat{J}_{j}-\hat{K}_{j}
$$

The operators $\hat{J}_{j}$ and $\hat{K}_{j}$ are the Coulomb and exchange operators respectively.

The Hartree-Fock equations may also be expressed over spatial orbitals, along with the Hartree-Fock energy. Assuming that all molecular orbitals (MOs) are doubly occupied, which is referred to as a closed-shell system, the Hartree-Fock energy expression is

$$
E_{\mathrm{HF}}=\sum_{a=1}^{N / 2} h_{a a}+\sum_{a=1}^{N / 2} \sum_{b=1}^{N / 2} 2 J_{a b}-K_{a b}
$$

The one-electron energy is given by $h_{a a}=\left\langle\phi_{a}|\hat{h}| \phi_{a}\right\rangle$, and the two-electron energy is given by the Coulomb, $J_{a b}$, and exchange, $K_{a b}$, energies.

$$
\begin{aligned}
J_{a b} & =\iint \phi_{a}^{*}\left(\mathbf{r}_{1}\right) \phi_{b}^{*}\left(\mathbf{r}_{2}\right) \frac{1}{r_{12}} \phi_{a}\left(\mathbf{r}_{1}\right) \phi_{b}\left(\mathbf{r}_{2}\right) d \mathbf{r}_{1} d \mathbf{r}_{2}=\langle a b \mid a b\rangle \\
K_{a b} & =\iint \phi_{a}^{*}\left(\mathbf{r}_{1}\right) \phi_{b}^{*}\left(\mathbf{r}_{2}\right) \frac{1}{r_{12}} \phi_{b}\left(\mathbf{r}_{1}\right) \phi_{a}\left(\mathbf{r}_{2}\right) d \mathbf{r}_{1} d \mathbf{r}_{2}=\langle a b \mid b a\rangle
\end{aligned}
$$


The closed-shell Hartree-Fock equations have the same form as the spin orbital case,

$$
\hat{f} \phi_{a}\left(\mathbf{r}_{1}\right)=\epsilon_{a} \phi_{a}\left(\mathbf{r}_{1}\right)
$$

however, the closed-shell Fock operator is given by

$$
\hat{f}=\hat{h}+\sum_{b=1}^{N / 2} 2 \hat{J}_{b}-\hat{K}_{b}
$$

The closed-shell Hartree-Fock equations can be solved by expanding the molecular orbitals as a linear combination of basis functions.

$$
\phi_{a}=\sum_{\mu=1}^{K} C_{\mu a} \psi_{\mu}
$$

Substitution of the expansion into the Hartree-Fock equations leads to,

$$
\sum_{\nu=1} C_{\nu a} \hat{f} \psi_{\nu}\left(\mathbf{r}_{1}\right)=\epsilon_{a} \sum_{\nu=1} C_{\nu a} \psi_{\nu}\left(\mathbf{r}_{1}\right)
$$

If both sides are multiplied by the complex conjugate of another basis function, $\psi_{\mu}^{*}\left(\mathbf{r}_{1}\right)$, and integrated with respect to $\mathbf{r}_{1}$, the result,

$$
\sum_{\nu=1} C_{\nu a} \int \psi_{\mu}^{*}\left(\mathbf{r}_{1}\right) \hat{f} \psi_{\nu}\left(\mathbf{r}_{1}\right) d \mathbf{r}_{1}=\epsilon_{a} \sum_{\nu=1} C_{\nu a} \int \psi_{\mu}^{*}\left(\mathbf{r}_{1}\right) \psi_{\nu}\left(\mathbf{r}_{1}\right) d \mathbf{r}_{1}
$$

is a matrix equation known as Roothaan's equation. Roothaan's equation in matrix form is expressed as

$$
F C=S C \epsilon
$$

where $C$ is the molecular orbital coefficient matrix, $F$ is the Fock matrix, $S$ is the overlap matrix and $\epsilon$ is a diagonal matrix of orbital energies. Each element of the overlap matrix, $S_{\mu \nu}$, is given by

$$
S_{\mu \nu}=\int \psi_{\mu}^{*}\left(\mathbf{r}_{1}\right) \psi_{\nu}\left(\mathbf{r}_{1}\right) d \mathbf{r}_{1}
$$


The elements of the Fock matrix, $F_{\mu \nu}$, are

$$
F_{\mu \nu}=\int \psi_{\mu}^{*}\left(\mathbf{r}_{1}\right) \hat{f} \psi_{\nu}\left(\mathbf{r}_{1}\right) d \mathbf{r}_{1}
$$

The Fock matrix cannot be constructed without prior knowledge of the molecular orbital coefficients, as the two-electron part of the Fock operator is dependent on the molecular orbitals. Therefore, the solution to Roothaan's equation first begins with a guess coefficient matrix. The matrix equation is converted to a standard eigenvalue equation by defining a new coefficient matrix, $C^{\prime}=X^{-1} C$, where the matrix $X$ transforms the basis to one in which the overlap matrix is the identity, $X^{+} S X=S^{\prime}=I$

$$
F X C^{\prime}=S X C^{\prime} \epsilon
$$

The above equation is multiplied on the left by $X^{+}$,

$$
X^{+} F X C^{\prime}=X^{+} S X C^{\prime} \epsilon
$$

which results in a standard eigenvalue equation which can be solved to give a new $C^{\prime}$ matrix.

$$
F^{\prime} C^{\prime}=C_{\epsilon}^{\prime}
$$

The matrix $C^{\prime}$, can be back transformed to give the coefficient matrix over the original basis and it may be used to construct a new Fock matrix. This procedure is continued until some convergence criteria on the energy or coefficients is achieved. Due to the iterative nature of the solution, this method is often referred to as the self-consistent field (SCF) approach.

A common value used to test convergence in a SCF approach is the density matrix, 
$P$. The density matrix is defined as the matrix product

$$
P=2 C C^{+}
$$

and is closely related to the electron density, $\rho(\mathbf{r})$, which is calculated as a sum over the products of MOs,

$$
\rho(\mathbf{r})=2 \sum_{a=1}^{N / 2} \phi_{a}^{*}(\mathbf{r}) \phi_{a}(\mathbf{r})
$$

The MOs can be expanded in terms of basis functions to give

$$
\begin{gathered}
\rho(\mathbf{r})=2 \sum_{a=1}^{N / 2} \sum_{\mu=1}^{K} \sum_{\nu=1}^{K} C_{\mu a}^{*} C_{\nu a} \psi_{\mu}^{*}(\mathbf{r}) \psi_{\nu}(\mathbf{r}) \\
\rho(\mathbf{r})=\sum_{\mu \nu} P_{\mu \nu} \psi_{\mu}^{*}(\mathbf{r}) \psi_{\nu}(\mathbf{r})
\end{gathered}
$$

where

$$
P_{\mu \nu}=2 \sum_{a=1}^{N / 2} C_{\mu a}^{*} C_{\nu a}
$$

is a density matrix element. The density matrix contains all the information necessary to calculate one-electron properties of the wave function of a molecule, corresponding to the specified basis set.

Experimentally observed properties, such as dipole moment and polarizabilities, are calculated as expectation values of the wave function, where the one-electron operator, $\widehat{\mathcal{O}}_{1}$, has the general form

$$
\widehat{\mathcal{O}}_{1}=\sum_{i=1}^{N} \hat{o}_{i}
$$

The expectation value of such an operator can be expressed in terms of integrals over basis functions and the density matrix,

$$
\left\langle\Psi_{\mathrm{HF}}\left|\widehat{\mathcal{O}}_{1}\right| \Psi_{\mathrm{HF}}\right\rangle=\sum_{\mu \nu} P_{\mu \nu}\left\langle\mu\left|\hat{o}_{1}\right| \nu\right\rangle
$$


In the case of electronic dipole moment, the one-electron operator, $\widehat{\mathcal{O}}_{1}$, is $\hat{\mathbf{r}}_{1}$, where $\hat{\mathbf{r}}_{1}=\left(\hat{x}_{1}, \hat{y}_{1}, \hat{z}_{1}\right)$. The electronic second moment is calculated using $\hat{\mathbf{r}}_{1}^{2}$. The components of electronic first and second moments are used to define a theoretical measure of shape, size and steric hindrance in Chapter 2. As a definition of molecular size and shape, expectation values of the Hartree-Fock wave function are quite sufficient, however, there is an inherent flaw in the Hartree-Fock wave function which leads to inexact one and two-electron expectation values and the incorrect energy.

\section{Electron Correlation}

The Hartree-Fock wave function is a Slater determinant of one-electron functions (Equation 1.7), and, as a consequence, it does not correctly describe the interaction between electrons. The Fock operator is an effective one-electron potential and hence each electron experiences the average field of the other electrons. The motion of electrons with parallel spins is correlated and accounted for by the exchange interaction. However, the motion of electrons with opposite spin is not correlated, and therefore, the probability of finding two electrons with opposite spins at the same point in space is not zero. ${ }^{4}$ The missing electron correlation results in an erroneous wave function in which the discrepancies from exact values depend on the system and property of interest. The difference in energy is referred to as the correlation energy, $E_{\text {corr }}$, and may be defined as

$$
E_{\text {corr }}=\mathcal{E}_{0}-E_{\mathrm{HFlim}}
$$

where $\mathcal{E}_{0}$ is the exact non-relativistic energy and $E_{\mathrm{HFlim}}$ is the Hartree-Fock limit energy. The Hartree-Fock limit is Hartree-Fock theory in the limit of a complete basis set (i.e. $K \rightarrow \infty$ ), and the energy at the Hartree-Fock limit is a lower bound 
to any finite basis Hartree-Fock energy. Generally, for light atoms, the correlation energy is less than $1 \%$ of the total electronic energy. However, this small percentage can be larger than bond energies, even for the smallest organic molecules. ${ }^{6}$ Such errors can lead to incorrect reaction energetics and kinetics and render Hartree-Fock theory useless for systems in which the correlation energy does not remain constant. There are several $a b$ initio electronic structure theories which exist to account for the missing electron correlation; some of them are discussed in the following two sections (Sections 3 and 4). In Chapter 6, possible new approaches to the correlation problem are introduced.

\section{Post Hartree-Fock Methods}

Many of the theories that account for the missing electron correlation, correlated theories, use the Hartree-Fock approximation as their basis. These theories are referred to as post Hartree-Fock methods. Conceptually, the simplest post Hartree-Fock method is configuration interaction(CI) theory. The basic idea behind $\mathrm{CI}$ is the use of a wave function which is a linear combination of Slater determinants describing different electronic configurations of the system (Equation 6.9).

$$
\left|\Psi_{\mathrm{CI}}\right\rangle=c_{0}\left|\Psi_{0}\right\rangle+\sum_{a r} c_{a}^{r}\left|\Psi_{a}^{r}\right\rangle+\sum_{\substack{a<b \\ r<s}} c_{a b}^{r s}\left|\Psi_{a b}^{r s}\right\rangle+\ldots
$$

where $a$ and $b$ denote occupied orbitals and $r$ and $s$ denote virtual orbitals. The Hartree-Fock wave function is $\left|\Psi_{0}\right\rangle$ and the following terms are the determinants generated by single, $\left|\Psi_{a}^{r}\right\rangle$, double, $\left|\Psi_{a b}^{r s}\right\rangle$, triple and higher excitations of the HartreeFock wave function, multiplied by the corresponding CI expansion coefficient, $c_{0}, c_{a}^{5}$, $c_{a b}^{r s}$, etc. 
An attractive property of $\mathrm{CI}$ is that it is variational, which means it obeys the variational principle. The variational principle is a fundamental theorem of quantum mechanics. The theorem states that for any well-behaved wave function, $|\Psi\rangle$, which satisfies the specified boundary conditions, the expectation value of the Hamiltonian is bounded from below by the lowest energy eigenvalue.

$$
\frac{\langle\Psi|\widehat{H}| \Psi\rangle}{\langle\Psi \mid \Psi\rangle} \geq \mathcal{E}_{0}
$$

The theorem implies that as the expectation value approaches $\mathcal{E}_{0},|\Psi\rangle$ approaches the exact ground state wave function $\left|\Phi_{0}\right\rangle$, where $\widehat{H}\left|\Phi_{0}\right\rangle=\mathcal{E}_{0}\left|\Phi_{0}\right\rangle$. Therefore, in the ground state, the energy of an approximate wave function is a measure of its quality. Hartree-Fock theory is variational. The quality of the Hartree-Fock wave function is improved, and the energy approaches the Hartree-Fock limit from above, as the size of the basis set is increased. In the case of CI, if the number of configurations in the CI wave function expansion is increased, the energy approaches the exact non-relativistic energy monotonically, or at least to the limit of the basis set.

The CI problem is the determination of the expansion coefficients, $c_{0}, c_{a}^{r}, c_{a b}^{r s}, \ldots$ If the CI wave function is substituted into the Schrödinger equation, where $\left\{\left|\Psi_{I}\right\rangle\right\}$ and $\left\{c_{I}\right\}$ represent the sets of all determinants and coefficients, the result is

$$
\mathrm{Hc}=\mathbf{E c}
$$

where $\mathbf{c}$ is the $\mathrm{CI}$ coefficient vector, $\mathbf{E}$ is a diagonal matrix of energies, and $\mathbf{H}$ is the CI matrix. Each element of the CI matrix is given by

$$
H_{I J}=\left\langle\Psi_{I}|\widehat{H}| \Psi_{J}\right\rangle
$$

The CI coefficients and energy of each configuration can be obtained by diagonalizing 
the CI matrix. When all possible configurations of a given system are included in the CI expansion, it is referred to as Full CI. For a given basis set, the difference between the Hartree-Fock energy and the Full CI energy is the basis set correlation energy. If the basis set is complete(i.e. infinite), which is called complete CI, the CI energy is the exact non-relativistic energy. However, this would mean constructing and diagonalizing a CI matrix of infinite dimension. In practice CI is truncated at different $n$-tuple excitations, such as CI with single and double excitations, CISD.

One problem with truncated CI methods, is that they are not size consistent. For a method to be size consistent, the energy of a collection of non-interacting molecules has to be equal to the sum of their individual energies. Consider a CI method, truncated at $n$-tuple excitations, applied to a system of two non-interacting molecules $(A \underset{\infty}{\longleftrightarrow} B)$ and the two molecules individually $(A+B)$. The energy of the individual molecules involves contributions from $n$-tuple excitations of each molecule, which actually corresponds to $2 n$-tuple excitations of the non-interacting system. Full $\mathrm{CI}$ is the only CI method that is size consistent. However, an approximation to Full CI that is size consistent is coupled-cluster theory.

The coupled-cluster approximation (CCA) involves expressing the wave function as a cluster expansion of the Hartree-Fock wave function, $\Psi_{0}$.

$$
\left|\Psi_{\mathrm{CC}}\right\rangle=e^{\hat{\mathcal{T}}}\left|\Psi_{0}\right\rangle
$$

The cluster operator, $\hat{\mathcal{T}}$, is a sum of operators, $\hat{\mathcal{T}}_{\boldsymbol{i}}$, which produce excited determinants from the Hartree-Fock wave function.

$$
\hat{\mathcal{T}}=\sum_{i=1}^{N} \hat{\mathcal{T}}_{i}
$$


For example, the doubles cluster operator, $\hat{\mathcal{T}}_{2}$, produces the different possible doubly excited determinants when it operates upon the Hartree-Fock wave function.

$$
\hat{\mathcal{T}}_{2}\left|\Psi_{0}\right\rangle=\frac{1}{4} \sum_{a b r s} c_{a b}^{r s}\left|\Psi_{a b}^{r s}\right\rangle
$$

Due to the exponential form of the cluster expansion of the wave function $\left(e^{\hat{T}}=\right.$ $\left.1+\hat{T}+\frac{\hat{T}^{2}}{2 l}+\ldots\right)$, the wave function also contains products of single excitations, double excitations, etc. The coupled-cluster approximation is based on the truncation of the cluster expansion of the wave function, normally at the doubles operator (CCSD). In CCSD, the quadruple excitations are approximated by the product of double excitations, $c_{a b c d}^{r s t u} \approx c_{a b}^{r s} * c_{c d}^{t u}$, where $c_{a b}^{r s} * c_{c d}^{t u}$ denotes the sum of all possible double excitation products which give the same quadruple excitation, while accounting for antisymmetry. The same approach can be extended to account for hextuple excitations as well. The most common form of coupled-cluster theory is the coupledcluster singles and doubles with approximate triples, $\operatorname{CCSD}(\mathrm{T}) \cdot{ }^{7}$ An approximation to the triples is used as opposed to including the triples operator, $\hat{\mathcal{T}}_{3}$, in the cluster expansion, CCSDT, due to the significant increase in scaling upon adding triple excitations. While CCA is size consistent, the method is not variational, as it is possible to obtain an energy lower than the exact non-relativistic energy using CCA.

A somewhat different post Hartree-Fock method is Møller-Plesset perturbation theory. Møller-Plesset theory applies Rayleigh-Schrödinger perturbation theory to the Hartree-Fock Hamiltonian, $\widehat{H}_{0}$, and its eigenfunctions, which are the HartreeFock wave function and the different possible excited determinants. A perturbation, $\hat{\mathcal{V}}$, which is the correction to the Hartree-Fock effective potential, $\hat{v}_{\mathrm{HF}}(i)$, is applied 
to the Hartree-Fock Hamiltonian to give a perturbed Hamiltonian, $\widehat{H}$.

$$
\widehat{H}=\widehat{H}_{0}+\hat{\mathcal{V}}
$$

where

$$
\hat{\mathcal{V}}=\sum_{i=1}^{N} \sum_{j<i} \frac{1}{r_{i j}}-\sum_{i=1}^{N} \hat{v}_{\mathrm{HF}}(i)
$$

and

$$
\hat{v}_{\mathrm{HF}}(i)=\sum_{j=1}^{N} \hat{J}_{j}(i)-\hat{K}_{j}(i)
$$

Expressions for the $n^{\text {th }}$ order energies are derived through use of an ordering parameter, $\lambda$. The ordering parameter, which is later set to unity, multiplies the perturbation, $\lambda \hat{\mathcal{V}}$, and the energy, $\mathcal{E}$, and the wave function, $|\Phi\rangle$, are expanded in a Taylor series in terms of the eigenvalues and eigenfunctions of the Hartree-Fock Hamiltonian, respectively.

$$
\begin{gathered}
\mathcal{E}=E^{(0)}+\lambda E^{(1)}+\lambda^{2} E^{(2)}+\ldots \\
|\Phi\rangle=\left|\Psi^{(0)}\right\rangle+\lambda\left|\Psi^{(1)}\right\rangle+\lambda^{2}\left|\Psi^{(2)}\right\rangle+\ldots
\end{gathered}
$$

Substitution of the expanded energy (Equation 1.47) and wave function (Equation 1.48) into the Schrödinger equation, along with $\widehat{H}=\widehat{H}_{0}+\lambda \hat{\mathcal{V}}$, and collection of terms of the same order $\lambda^{n}$ result in the $n^{\text {th }}$ order energy corrections, $E^{(n)}$, to the HartreeFock energy. However, it should be noted that the zeroth-order energy of MøllerPlesset theory is the sum of the Hartree-Fock orbital energies, not the Hartree-Fock energy. The Hartree-Fock energy is given by the sum of the zeroth and first order energies,

$$
E_{\mathrm{HF}}=E^{(0)}+E^{(1)}
$$


The first correction to the Hartree-Fock energy (i.e. correlation energy) is at second order, where the energy expression is

$$
E^{(2)}=\sum_{\substack{a<b \\ r<s}} \frac{|\langle a b \| r s\rangle|^{2}}{\epsilon_{a}+\epsilon_{b}-\epsilon_{\tau}-\epsilon_{s}}
$$

The energy expressions for higher order corrections are increasingly complex, involving higher order products of two-electron integrals over products of orbital energies. The order of energy correction, $n$, calculated using Møller-Plesset perturbation theory corresponds to the commonly used notation, MPn. Like CCA, the MPn theories are size consistent but not variational.

The post Hartree-Fock methods each have their own advantages and disadvantages with respect to each other; size consistency, variational, etc.. However, they all have a common drawback, and that is their computational cost. Each method requires the calculation of two-electron integrals over molecular orbitals, $\langle a b \mid r s\rangle$, which increases the scaling with respect to the number of basis functions $K$. Hartree-Fock theory formally scales as $K^{4}$, which corresponds to calculation of two-electron integrals over basis functions. Post Hartree-Fock methods such as CID and MP2 scale as $K^{5}$, whereas CISDT and MP5 scale as $K^{8}{ }^{7}$ Such increases in computational cost make systems which can be studied using Hartree-Fock quickly become very expensive or impossible to study at the post Hartree-Fock level.

\section{Density Functional Theory}

An alternative approach to wave function theories, such as the Hartree-Fock and post Hartree-Fock approaches, is density functional theory (DFT). The formulation of DFT comes from expressing the energy of a system of electrons, $E$, as a functional 
of the electron density, $\rho(\mathbf{r})$.

$$
E[\rho(\mathbf{r})]=V_{\text {ext }}[\rho(\mathbf{r})]+F[\rho(\mathbf{r})]
$$

The energy functional is divided into two parts, the external potential, $\hat{V}_{\text {ext }}$, and the universal functional, $F[\rho(\mathbf{r})]$. In the case of a molecule in the absence of an external field, the external potential is the attraction of nuclei, $\hat{V}_{\text {ext }}=\hat{V}_{\mathrm{Ne}}$. The universal functional consists of all interactions independent of the external field, the kinetic energy of the electrons, $T[\rho(\mathbf{r})]$, and the electron-electron potential energy, $V_{\circledast}[\rho(\mathbf{r})]$.

$$
F[\rho(\mathbf{r})]=T[\rho(\mathbf{r})]+V_{\mathrm{ee}}[\rho(\mathbf{r})]
$$

The foundation of DFT was laid by two theorems proven by Hohenberg and Kohn ${ }^{8}$ Both theorems apply to the electron density of a non-degenerate ground state. The first theorem concerns the existence of an external potential which is uniquely determined by the ground state electron density, $\rho_{0}(\mathbf{r})$, and consequently determines the ground state wave function, $\Psi_{0}$, and energy, $E_{0}$, as well as other properties. The second theorem is the density functional analogue to the wave function variational principle, which states that only the true ground state density, $\rho_{0}(r)$, minimizes the energy functional, $E[\rho(\mathbf{r})]$. The second theorem has the conditions that the density is non-negative, $\rho(\mathbf{r}) \geq 0$, and integrates to the number of electrons, $\int \rho(\mathbf{r}) d \mathbf{r}=$ $N$. Both theorems contain the more complex condition that the density must be $v$-representable. For a given density to be $v$-representable, it must correspond to a external potential that is associated with an antisymmetric wave function. ${ }^{9}$ However, Levy ${ }^{10}$ proved both theorems using a constrained search approach which no longer requires the ground state to be non-degenerate. Also, Levy's reformulation of the 
Hohenberg-Kohn theorems relaxes the constraint of $v$-representability to requiring the density be $N$-representable. If the density is $N$-representable, it is associated with an antisymmetric wave function, but there is no requirement that there must be a corresponding external potential. While these theorems and their modifications provide a sound basis for DFT, there still remains the practical issue of minimizing the energy functional.

The energy functional of DFT is normally minimized using the Kohn-Sham approach. The Kohn-Sham approach involves defining a system of non-interacting electrons and their corresponding orbitals, which have the same electron density as the exact electron density, $\rho^{\mathrm{KS}}(\mathbf{r})=\rho_{0}(\mathbf{r})$. A system of non-interacting electrons is exactly described by a Slater determinant(Equation 1.7) of Kohn-Sham orbitals, $\left\{\chi_{i}^{\mathrm{KS}}\right\}$, and the electron density is given by

$$
\rho_{0}(\mathbf{r})=\sum_{i=1}^{N} \chi_{i}^{\mathrm{KS} *}(\mathbf{r}) \chi_{i}^{\mathrm{KS}}(\mathbf{r})
$$

The energy functional, using the Kohn-Sham approach, is

$$
\begin{aligned}
E_{0}=- & \sum_{A=1}^{M} Z_{A} \int \frac{\rho\left(\mathbf{r}_{1}\right)}{r_{1 A}} d \mathbf{r}_{1}-\frac{1}{2} \sum_{i=1}^{N}\left\langle\chi_{i}^{\mathrm{KS}}(1)\left|\nabla_{1}^{2}\right| \chi_{i}^{\mathrm{KS}}(1)\right\rangle \\
& +\frac{1}{2} \iint \frac{\rho\left(\mathbf{r}_{1}\right) \rho\left(\mathbf{r}_{2}\right)}{r_{12}} d \mathbf{r}_{1} d \mathbf{r}_{2}+E_{x c}[\rho(\mathbf{r})]
\end{aligned}
$$

The energy due to the external potential, or the nucleus-electron attraction potential energy, is given by

$$
V_{\mathrm{Ne}}[\rho(\mathbf{r})]=-\sum_{A=1}^{M} Z_{A} \int \frac{\rho\left(\mathbf{r}_{1}\right)}{r_{1 A}} d \mathbf{r}_{1}
$$

and the kinetic energy is written in terms of the Kohn-Sham orbitals,

$$
T[\rho(\mathbf{r})]=-\frac{1}{2} \sum_{i=1}^{N}\left\langle\chi_{i}^{\mathrm{KS}}(1)\left|\nabla_{1}^{2}\right| \chi_{i}^{\mathrm{KS}}(1)\right\rangle
$$


Part of the electron-electron potential energy is given by the classical Coulomb energy,

$$
J[\rho(\mathbf{r})]=\frac{1}{2} \iint \frac{\rho\left(\mathbf{r}_{1}\right) \rho\left(\mathbf{r}_{2}\right)}{r_{12}} d \mathbf{r}_{1} d \mathbf{r}_{2}
$$

The remainder of the electron-electron potential energy, which is the exchange and correlation, and the corrections to the kinetic and Coulomb energies are contained in the exchange-correlation energy term, $E_{x c}[\rho(\mathbf{r})]$.

$$
E_{x c}[\rho(\mathbf{r})]=\Delta T[\rho(\mathbf{r})]+\Delta V_{e e}[\rho(\mathbf{r})]
$$

The kinetic energy correction, $\Delta T[\rho(\mathbf{r})]$, accounts for the fact that the kinetic energy of the Kohn-Sham orbitals is for the non-interacting system, which is not equivalent to the kinetic energy of the true system. ${ }^{9}$ The correction to the electron-electron potential energy, $\Delta V_{e e}[\rho(\mathbf{r})]$, includes the exchange energy, the correlation energy, and the correction for the self-interaction present in the Coulomb energy. The selfinteraction error is demonstrated by the fact that a one electron system would have a Coulomb energy (Equation 1.57) not equal to zero. In Hartree-Fock theory, there is no self-interaction due to the cancellation of the Coulomb integral between a spin orbital and itself by the corresponding exchange integral, $J_{i i}-K_{i i}=0$ (Equation $1.9)$.

Similar to Hartree-Fock theory, if the Kohn-Sham energy expression (Equation 1.54 ) is minimized with respect to the Kohn-Sham orbitals, which define the density, under the constraint that they remain orthonormal, the resulting OEL equations are referred to as the Kohn-Sham equations.

$$
\hat{k} \chi_{i}^{\mathrm{KS}}\left(\mathbf{r}_{1}\right)=\epsilon_{i} \chi_{i}^{\mathrm{KS}}\left(\mathbf{r}_{1}\right)
$$


where $\hat{k}$ is the Kohn-Sham one-electron operator.

$$
\hat{k}=-\frac{1}{2} \nabla_{1}^{2}-\sum_{A=1}^{M} \frac{Z_{A}}{r_{1 A}}+\int \frac{\rho\left(\mathbf{r}_{2}\right)}{r_{12}} d \mathbf{r}_{2}+\hat{v}_{x c}\left(\mathbf{r}_{1}\right)
$$

The exchange correlation operator, $\hat{v}_{x c}(\mathbf{r})$, is defined as the functional derivative of the exchange-correlation energy with respect to the density.

$$
\hat{v}_{x c}(\mathbf{r})=\frac{\delta E_{x c}[\rho(\mathbf{r})]}{\delta \rho(\mathbf{r})}
$$

Also like Hartree-Fock, the Kohn-Sham one-electron operator, $\hat{k}$, depends on the electron density and therefore the Kohn-Sham equations have to be solved iteratively. Generally, the Kohn-Sham equations are solved in the same manner as the HartreeFock equations. The Kohn-Sham orbitals are expanded over basis functions and a matrix of Kohn-Sham operator elements, $K_{\mu \nu}$, is calculated and diagonalized. The electron-nucleus attraction, kinetic, and Coulomb elements are calculated as integrals over basis functions as in Hartree-Fock theory. However, the integrals involving the exchange-correlation operator are often complex and do not have an analytic expression. In such cases, the exchange-correlation contribution is calculated using numerical integration. The method of implementation of DFT through use of Kohn-Sham orbitals suggests an approach to calculating the electronic structure of a molecule that scales on the same order as Hartree-Fock and accounts for electron correlation. However, this is not entirely true.

The Kohn-Sham equations themselves are exact, and their solution, the KohnSham orbitals give the exact ground state density. However, the form of the exact exchange-correlation operator is unknown. As a consequence, while the general formulation of DFT is variational, the method is not variational when an approximate 
exchange-correlation operator is employed. There are several forms of the operator that are used in applications of DFT today. Conventionally the name of the exchangecorrelation functional is written in the form exchange functional name-correlation functional name. For example, the B3LYP functional is comprised of the Becke three parameter exchange functional, B3, and the Lee-Yang-Parr correlation functional, LYP (see Appendix A). Research continues into developing new exchange-correlation functionals, with some success. ${ }^{11}$

\section{Molecular Mechanics}

Molecular mechanics is a drastically different approach to modelling molecular systems than the wave function and density functional electronic structure theories discussed in the previous sections. Molecular mechanics empirically models bonding and non-bonding interactions between molecules using a variety of functions of distances, angles, and torsions. The theoretical foundation of molecular mechanics is rooted in the Born-Oppenheimer approximation. It is assumed that for each configuration of nuclei, or molecular geometry, the electrons find their optimum distribution and the energy is then a function of the geometry. However, molecular mechanics was first developed by fitting and attempting to predict and explain experimental observations.

The idea of molecular mechanics was first introduced by D.H. Andrews in $1930,{ }^{12}$ with five postulates on "The Nature of the Mechanical System in the Molecule", which he used to qualitatively predict Raman spectra. It was not until 1946 that attempts were made to use such ideas for quantitative purposes. ${ }^{13}$ Hill presented an equation for the "steric energy" of a molecule which had a van der Waals term and bond 
stretching and bending terms. ${ }^{14}$ He suggested that such an approach could be used to study polar and steric effects in molecules and that calculations could be correlated with experimental values of gas-phase reaction rates. Around the same time, Westheimer and Mayer were using similar equations to study the rates of racemization of optically active derivatives of biphenyl, including deriving equations for the vibrational frequencies of the planar forms of these molecules. ${ }^{15-17}$ Also at the same time, Dostrovsky et al. derived similar equations while studying steric and polar effects on $\mathrm{S}_{\mathrm{N}} 2$ reactions. ${ }^{18,} 19$ It was all these studies that led to the development of modern molecular mechanics.

In conventional molecular mechanics, the total energy of a molecule is divided into different components; bond stretching, $E_{\mathrm{s}}$, angle bending, $E_{\mathrm{b}}$, torsional interactions, $E_{\text {tor }}$, van der Waals interactions, $E_{\mathrm{vdW}}$, and electrostatic interactions, $E_{\text {elec }}{ }^{20}$

$$
E_{\mathrm{tot}}(\mathbf{R})=E_{\mathrm{s}}+E_{\mathrm{b}}+E_{\mathrm{tor}}+E_{\mathrm{vdW}}+E_{\text {elec }}
$$

Some formulations may use other energy terms to describe phenomena, such as hydrogen bonding, that are not described well by the existing terms. The functional form of these energy terms and the empirical parameters that occur in the functions are known as a force field. In the case of bond stretching, the functional form is often a quadratic.

$$
E_{\mathrm{s}}=\frac{1}{2} k_{\mathrm{s}}\left(l-l_{0}\right)^{2}
$$

where $l$ is the actual bond length, $l_{0}$ is the natural bond length, and $k_{\mathrm{s}}$ is the empirical force constant. The behaviour of a chemical bond is modelled quite well with a quadratic potential for bond lengths very near the natural bond length. However, once a bond is lengthened significantly it is known to exhibit anharmonic behaviour. 
In several molecular mechanics methods, this anharmonicity is treated by adding cubic and even quartic terms. Bond angles are treated in a similar manner. For small changes in angles, a harmonic potential works well, but for large angle changes higher order terms are required. For torsional interactions, bond rotation, functions with cosine terms are used to describe the interactions.

$$
E_{\text {tor }}=k_{\phi}(1+\cos (\phi))
$$

where $\phi$ is the torsional angle and $k_{\phi}$ is the force constant. The functional form of torsional interactions often has several terms, which in accordance with spectroscopy, follows the form of a Fourier series. ${ }^{20}$ In the case of van der Waals interactions, a modified form of the Lennard-Jones potential ${ }^{21}$ is normally used.

$$
E_{\mathrm{vdW}}=4 \epsilon\left[\left(\frac{\sigma}{R}\right)^{12}-\left(\frac{\sigma}{R}\right)^{6}\right]
$$

where $R$ is the interatomic distance, $\sigma$ is the finite distance of zero potential, and $\epsilon$ is the depth of the well. Electrostatic interactions are often modelled simply using point charges and Coulomb's law, ${ }^{20}$

$$
E_{\text {elec }}=\frac{q_{A} q_{B}}{D R_{A B}}
$$

where $q_{A}$ and $q_{B}$ are atomic charges, $R_{A B}$ is the interatomic distance, and $D$ is the effective dielectric constant of the solvent. However, the function may be modified to include dipole-dipole and point charge-dipole interactions. Once the functional form of a molecular mechanics force field is defined, the empirical parameters can be defined through fitting of experimental or $a b$ initio data.

Molecular mechanics methods are significantly less computationally expensive than Hartree-Fock theory, even when the most elaborate force fields are used. Such 
computational speed allows for the study of extremely large systems, such as proteins and nanoclusters, and their dynamics. These models and simulations allow for the extraction of data which is currently impossible to obtain for such systems using electronic structure methods. The development of force fields for specific systems leads to molecular mechanics models that agree quite well with experiment. However, when force fields are applied to systems for which they were not trained, the results are normally unreliable. A new approach to empirically modelling molecular systems is introduced in Chapter 5, which includes the electrons in the energy expression.

The development of Simulated Electronic Structure Theory, which is discussed in Chapter 5, is a result of deriving new properties of the Hartree-Fock wave function. These new properties are the average interparticle distances; the average distance between two electrons and the average distance between an electron and a nucleus. Such properties are derived from first and second moment operators (Section 1) and are found to be related to the molecular orbital energy components of the Hartree-Fock wave function, Chapters 3 and 4 . These relationships then lead to the development of Simulated Electronic Structure Theory. However, the first and second moment operators are first used to define a theoretical measure of the size and shape of a molecule or substituent, Chapter 2. Yet, the average distance between two electrons was first derived in an effort to create a new approach to the correlation problem which is discussed in Chapter 6. 


\section{References}

(1) D. Röthlisberger, O. Khersonsky, A. M. Wollacott, L. Jiang, J. DeChancie, J. Betker, J. L. Gallaher, E. A. Althoff, A. Zanghellini, O. Dym, S. Albeck, K. N. Houk, D. S. Tawfik, and D. Baker. Nature, 453:190-197, 2008.

(2) Z. Rolik, A. Szabados, and P. R. Surján. J. Chem. Phys., 128:144101, 2008.

(3) M. Born and R. Oppenheimer. Ann. Physik, 84:457-484, 1927.

(4) A. Szabo and N. S. Ostlund. Modern Quantum Chemisty: Introduction to Advanced Electronic Structure Theory. Dover Publications, Inc., first edition, 1996.

(5) R. K. Nesbet. Phys. Rev. A, 62:040701, 2000.

(6) I. N. Levine. Quantum Chemistry. Prentice-Hall, Inc., fifth edition, 2001.

(7) C. J. Cramer. Essentials of Computational Chemistry: Theories and Models. Jon Wiley \& Sons, Ltd., second edition, 2004.

(8) P. Hohenberg and W. Kohn. Phys. Rev., 136:B864-B871, 1964.

(9) W. Koch and M. C. Holthausen. A Chemist's Guide to Density Functional Theory. Wiley-VCH, second edition, 2001.

(10) M. Levy. Proc. Natl. Acad. Sci. USA, 76:6062-6065, 1979.

(11) Y. Zhao and D. G. Truhlar. Theo. Chem. Acc., 120:215-241, 2008.

(12) D. H. Andrews. Phys. Rev., 36:544-554, 1930. 
(13) U. Burkert and N. L. Allinger. Molecular Mechanics. American Chemical Society, first edition, 1982.

(14) T. L. Hill. J. Chem. Phys., 14:465, 1946.

(15) F. H. Westheimer and J. E. Mayer. J. Chem. Phys., 14:733-738, 1946.

(16) F. H. Westheimer. J. Chem. Phys., 15:252-260, 1947.

(17) M. Rieger and F. H. Westheimer. J. Am. Chem. Soc., 72:19-28, 1950.

(18) I. Dostrovsky, E. D. Hughes, and C. K. Ingold. J. Chem. Soc., pages 173-194, 1946.

(19) P. B. D. de la Mare, L. Fowden, E. D. Hughes, C. K. Ingold, and J. D. H. Mackie. J. Chem. Soc., pages 3200-3236, 1955.

(20) J. P. Bowen and N. L. Allinger. Molecular mechanics: The art and science of parameterization. Reviews in Computational Chemistry, 2:81-97, 1991.

(21) L. E. Lennard-Jones. Proc. Phys. Soc., 43:461-482, 1931. 
The following Chapter, Chapter 2, is published as an article in the Journal of Physical Chemistry A.

J. W. Hollett, A. Kelly, and R. A. Poirier, J. Phys. Chem. A, 110:13884-13888, 2006.

J. W. Hollett is the principal author and performed the majority of the research under the supervision of R. A. Poirier. 


\section{Chapter 2}

\section{Quantum mechanical size and steric hindrance}

\section{Introduction}

The shape and size of a molecule or substituent can be very influential on its physical and chemical properties. A predominant topic concerning substituent shape and size is steric hindrance. There are various methods that quantify steric effects, however the result often varies with the method. While geometry provides important insight into how a substituent may interact sterically, it does not provide a quantitative measure of steric interaction. Therefore, most methods quantify the steric effects of substituents in terms of energy. The first steric constant, $E_{S}$, was defined by Taft ${ }^{1}$ from a modified version of the Hammett equation. ${ }^{2}$

$$
E_{S}=\log \left(\frac{k_{X}}{k_{0}}\right)
$$


Taft's $E_{S}$ values are determined from the reaction rates, $k_{X}$, of acid hydrolysis of substituted aliphatic esters, $X \mathrm{COOR}$, and the reaction rate, $k_{0}$, of acid hydrolysis of the ester $\mathrm{CH}_{3} \mathrm{COOR}$ averaged over four different reaction conditions. ${ }^{3}$ However, soon after the introduction of $E_{S}$, it was noticed that electronic effects, such as polar and resonance effects, were included in the steric constant. Various modifications have been made to Taft's $E_{S}$ in effort to eliminate electronic effects and determine steric constants which are more generally applicable. ${ }^{3}$ Dubois defined the Taft-Dubois steric parameter $E_{S}^{\prime}$ in the same manner as $E_{S}$, however the rates were measured using only one standard reaction, the acid catalyzed esterfication of carboxylic acids at $40^{\circ} \mathrm{C}$ in methanol. ${ }^{4}$ Hancock et al. modified the definition of $E_{S}$ to account for the effect of hyperconjugation, ${ }^{3}$

$$
E_{s}^{c}=E_{s}+0.306(N-3),
$$

where $N$ is the number of $\alpha$-hydrogens. Another modification of $E_{S}$ was presented by Unger and Hansch. They define $E_{S}^{e}(X)=E_{S}\left(\mathrm{CH}_{2} X\right)$, which corrects for electronic contributions. ${ }^{3}$ While these steric parameters are widely used, they are defined from a single type of reaction and hence are only moderately successful at predicting steric effects in general.

An alternative method to determining the steric effect of a substituent is through the energetics of conformational processes. The advantage of such an approach is that it avoids many other effects of a substituent that may be encountered in a chemical reaction, which are impossible to exclude. The simplest example is Anderson's $P$ values which involve the rotation around the C-C bond in ethanes. ${ }^{5}$ However, bond length and geometry can have a large influence on the lateral interaction between 
the substituent of interest and the substituents on the neighbouring carbon atom, which leads to unreliable steric information. Similar disadvantages are shared with $A$-values. ${ }^{6}$ The $A$-value for a substituent $X$ is defined as

$$
A \text {-value }\left(\mathrm{kcal} \mathrm{mol}^{-1}\right)=-\Delta G^{\circ}=\frac{R T \ln K}{1000},
$$

where $K$ is the equilibrium constant for the equatorial and axial isomers of the monosubstituted cyclohexane. Like $P$-values, factors such as bond length and the shape of the substituent lead to $A$-values that do not always correlate well with the spatial requirement of the substituent. A solution to such a problem may be the design of a conformational process which involves a more multi-dimensional interaction with the substituent, such as the topomerization process shown in Figure 2.1. ${ }^{7}$ The ring

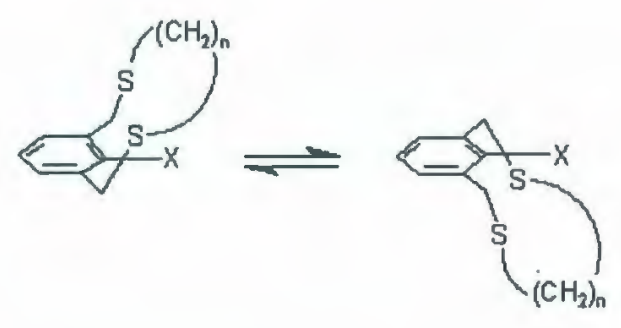

Figure 2.1. Topomerization of phane system with intra-annular substituent, $X$, used to determine $n$-values. ${ }^{7}$

inversion of this system is used to determine the steric parameter, $n$-value ${ }^{7}$ of $X$. The $n$-value is defined as the value of $n$ such that $\Delta \mathrm{G}_{c}^{\ddagger}=15 \mathrm{kcal} \mathrm{mol}^{-1}$. While the idea behind the $n$-value as a measure of the size of a substituent is promising, the application does have flaws. The determination of the $n$-values involves interpolation or extrapolation to $f(n)=\Delta \mathrm{G}_{c}^{\ddagger}=15 \mathrm{kcal} \mathrm{mol}^{-1}$, approximating $f(n)$ as linear. Yet, despite the flaws and small number of data points, relatively good results can be 
obtained. ${ }^{7}$ While there are various methods to quantify the spatial requirement of a substituent, steric hindrance does not necessarily correlate perfectly with the shape, and size or volume of a substituent.

Perhaps the oldest and most popular method to determine the volume of a molecule is through the atoms in molecules approach. By using the average radii of atoms in different environments, ${ }^{8,9}$ determined by kinetic gas theory or X-ray crystallography, the shape and volume of molecules can be approximated. ${ }^{8}$ Since the idea was introduced in the 1960 s, there have been several algorithms developed for the calculation of the molecular volumes of many different types of compounds. One of the more recent algorithms, by Gavezzotti, ${ }^{10}$ is capable of dealing with complicated structures such as cage compounds and inclusion compounds in crystalline matrices. These empirical calculations are widely used. However, the use of quantum mechanics to calculate molecular volumes is an alternative. In 1967 Bader et al. determined the size and shape of first row homonuclear diatomic molecules from outer contours of the Hartree-Fock electron density distribution. ${ }^{11}$ Once the theory of atoms in molecules had advanced, such as the aforementioned volume algorithms, along with computational methods, determining molecular shapes and sizes was once again investigated by Bader et al. ${ }^{12}$ The molecular shapes and volumes agreed well with the corresponding van der Waals volumes using isodensity contours of 0.001 a.u. and 0.002 a.u.. It was also found that the properties of various atoms and functional groups were transferable from molecule to molecule, especially in the case of normal hydrocarbons. ${ }^{12}$ Another method of calculating size using quantum mechanics was introduced by Csizmadia et al. ${ }^{13}$ when studying localized molecular orbitals (LMOs). A theoretical 
definition of the size of an electron pair, $\left\langle r_{1}^{2}\right\rangle_{R_{a}}$, is given as

$$
\left\langle r_{1}^{2}\right\rangle_{R_{a}}=\left|\left\langle\psi_{a}\left|r_{1}^{2}\right| \psi_{a}\right\rangle_{0}-R_{a}^{2}\right|
$$

where $R_{a}$ is the centroid of charge of the LMO,

$$
\left|R_{a}\right|=\sqrt{\left\langle\psi_{a}\left|x_{1}\right| \psi_{a}\right\rangle_{0}^{2}+\left\langle\psi_{a}\left|y_{1}\right| \psi_{a}\right\rangle_{0}^{2}+\left\langle\psi_{a}\left|z_{1}\right| \psi_{a}\right\rangle_{0}^{2}}
$$

calculated at some point $R_{0}$. While this definition cannot describe entire substituents, it can be successfully applied to bonds and lone pairs. ${ }^{13}$ Later Csizmadia focused on the components of $\left\langle r^{2}\right\rangle,\left\langle x^{2}\right\rangle,\left\langle y^{2}\right\rangle$, and $\left\langle z^{2}\right\rangle$, to define the shape of an electron pair. ${ }^{14}$ The second moment tensor of an LMO with respect to its own centroid of charge was defined as,

$$
\Omega=\left(\begin{array}{ccc}
{\left[\left\langle x^{2}\right\rangle_{0}-\langle x\rangle_{0}^{2}\right]} & {\left[\langle x y\rangle_{0}-\langle x\rangle_{0}\langle y\rangle_{0}\right]} & {\left[\langle x z\rangle_{0}-\langle x\rangle_{0}\langle z\rangle_{0}\right]} \\
& {\left[\left\langle y^{2}\right\rangle_{0}-\langle y\rangle_{0}^{2}\right]} & {\left[\langle y z\rangle_{0}-\langle y\rangle_{0}\langle z\rangle_{0}\right]} \\
& {\left[\left\langle z^{2}\right\rangle_{0}-\langle z\rangle_{0}^{2}\right]}
\end{array}\right)
$$

It is suggested that if the tensor is diagonalized,

$$
U^{+} \Omega U=\left(\begin{array}{ccc}
\left\langle x^{\prime 2}\right\rangle & 0 & 0 \\
& \left\langle y^{\prime 2}\right\rangle & 0 \\
& & \left\langle z^{\prime 2}\right\rangle
\end{array}\right)
$$

then the diagonal values, $\left\langle x^{2}\right\rangle,\left\langle y^{2}\right\rangle$, and $\left\langle z^{2}\right\rangle$ are aligned with the axes of an ellipsoid which describes the shape of the LMO. A similar calculation could be applied to an entire molecule. Analogous to the definition of the second moment tensor of an electron pair, the second moment tensor of a molecule can be defined so that when diagonalized, the diagonal values are the major and minor axes of an ellipsoid in which the molecule is inscribed. The process can then be taken a step further, by 
calculating the average radius of the ellipsoid and its volume. Such an approximation to molecular volume is calculated from expectation values of the Hartree-Fock wave function and is much less time consuming than tracing a three-dimensional contour around a molecule. The radii and volumes determined for different substituents can then be used to predict steric effects.

\section{Theory}

\subsection{The origin invariant electronic second moment tensor of a molecule}

The second moment tensor of a molecule calculated at some point $r_{0}=\left(x_{0}, y_{0}, z_{0}\right)$, is given as

$$
\mathcal{S}=\left(\begin{array}{ccc}
\left\langle x^{2}\right\rangle_{r_{0}} & \langle x y\rangle_{r_{0}} & \langle x z\rangle_{r_{0}} \\
& \left\langle y^{2}\right\rangle_{r_{0}} & \langle y z\rangle_{r_{0}} \\
& & \left\langle z^{2}\right\rangle_{r_{0}}
\end{array}\right)
$$

Expectation values of the form $\langle i j\rangle, i=x, y$, or $z$ and $j=x, y$, or $z$, are defined as follows,

$$
\langle i j\rangle=\int \Psi^{*}(r) i j \Psi(r) d r
$$

where $\Psi$ is the Hartree-Fock wave function. It is seen that the second moment tensor depends on the origin and hence a standard origin should be chosen, such as the center of nuclear charge. However, the second moment tensor can become origin invariant if it is defined in the following manner,

$$
\tilde{\mathcal{S}}=\left(\begin{array}{ccc}
\left\langle\widetilde{x^{2}}\right\rangle & \langle\widetilde{x y}\rangle & \langle\widetilde{x z}\rangle \\
& \left\langle\widetilde{y^{2}}\right\rangle & \langle\widetilde{y z}\rangle \\
& & \left\langle\widetilde{z^{2}}\right\rangle
\end{array}\right)
$$


where

$$
\langle\tilde{i j}\rangle=\langle i j\rangle_{r_{a}}-\frac{\langle i\rangle_{r_{a}}\langle j\rangle_{r_{a}}}{N} \quad i=x, y \text {, or } z \text { and } j=x, y \text { or } z
$$

$N$ is the number of electrons and $r_{a}$ is an arbitrary origin. The tensor $\widetilde{\mathcal{S}}$ is a real symmetric matrix and therefore can be diagonalized yielding a principal coordinate system.

$$
\begin{aligned}
& Q^{+} \widetilde{\mathcal{S}} Q=\tilde{\mathcal{S}}^{\prime} \\
& \widetilde{\mathcal{S}}^{\prime}=\left(\begin{array}{ccc}
\left.\widetilde{x^{2}}\right\rangle & 0 & 0 \\
& \left.\widetilde{y^{\prime 2}}\right\rangle & 0 \\
& & \left.\widetilde{z^{\prime 2}}\right\rangle
\end{array}\right)
\end{aligned}
$$

The eigenvalues, $\left.\left.\widetilde{\left\langle x^{\prime 2}\right.}\right\rangle, \widetilde{\left\langle y^{\prime 2}\right.}\right\rangle$ and $\left.\widetilde{z^{\prime 2}}\right\rangle$, correspond to the principal axes of the electronic second moment of the molecule, $Q$, and can be associated with the major and minor axes of an ellipsoid. The geometric average, $\widetilde{R}$, of these axes is given by

$$
\left.\left.\widetilde{R}=\left(\widetilde{\left\langle x^{\prime 2}\right.}\right\rangle\left\langle\widetilde{y^{\prime 2}}\right\rangle \widetilde{z^{2}}\right\rangle\right)^{1 / 6}
$$

and the volume of the ellipsoid, $\widetilde{V}$, is

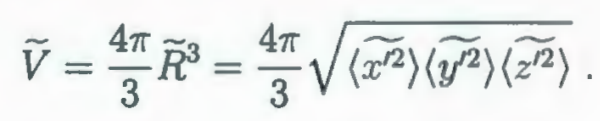

\section{Method}

Calculation of the origin invariant second moment tensor, and diagonalization were performed using the MUNgauss program. ${ }^{15}$ All calculations, with the exception of $\mathrm{HI}$, were performed at $\mathrm{HF} / 6-31 \mathrm{G}(\mathrm{d}) / / \mathrm{HF} / 6-31 \mathrm{G}(\mathrm{d})$. The double zeta Huzinaga basis set for iodine ${ }^{16}$ was used for calculations on HI. Geometries were optimized using Gaussian03. ${ }^{17}$ 


\section{Results and Discussion}

\subsection{Molecular Shape and Volume}

The eigenvalues of the second moment tensor, $\widetilde{\mathcal{S}}$, the average radius, $\widetilde{R}$, and the molecular volume, $\widetilde{V}$, for each molecule studied are given in Table 2.1.

In an effort to investigate the validity of this method, the radii of the hydrides were compared to the corresponding van der Waals ${ }^{8}$ and Bragg-Slater ${ }^{9}$ atomic radii (Figure 2.2). The average radii of the hydrides correlate very well with both the

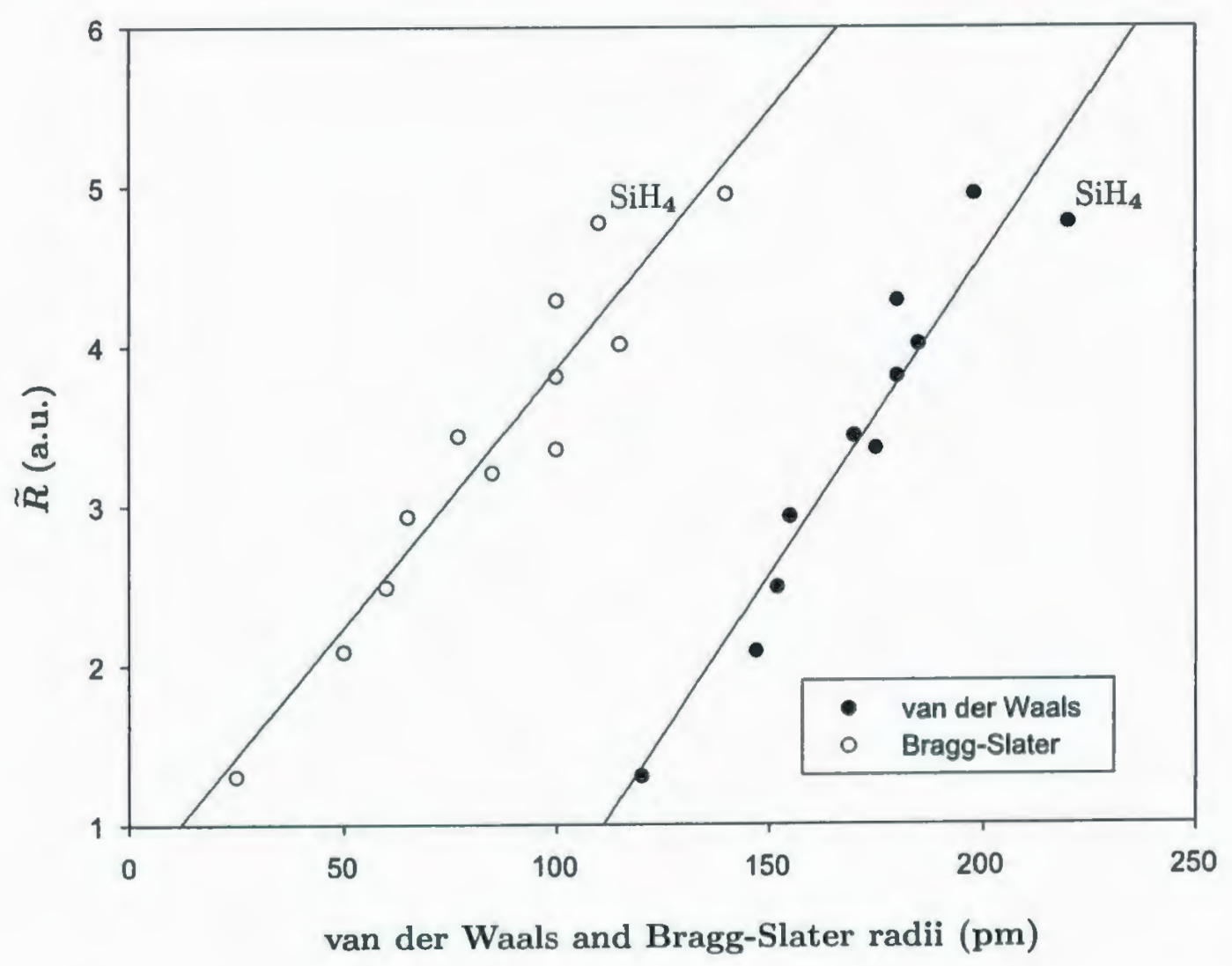

Figure 2.2. Comparison of average radii of hydrides to van der Waals and Bragg-Slater radii 
van der Waals $\left(R^{2}=0.916\right)$ and Bragg-Slater $\left(R^{2}=0.910\right)$ atomic radii. Most of the observed scatter is due to the number of hydrogens bonded to each atom. Negative deviations are seen with $\mathrm{HF}, \mathrm{HCl}$ and $\mathrm{HBr}$, whereas $\mathrm{NH}_{3}, \mathrm{CH}_{4}$, and $\mathrm{PH}_{3}$ deviate positively. An exception to this trend is the van der Waals radius of $\mathrm{Si}$, which is underestimated by $\widetilde{R}$ of $\mathrm{SiH}_{4}$, although, when comparing $\widetilde{R}$ to the Bragg-Slater radius, the expected positive deviation is seen. While the radii predicted from the electronic second moment have been compared to experimentally determined values, the molecular volumes will be compared to an alternative computational method.

The molecular volume approximated by the second moment ellipsoid, $\widetilde{V}$, and the molecular volumes enclosed by isodensity contours $(\rho=0.001$ and $\rho=0.002$ a.u. $),{ }^{12}$ $V_{\rho}$, were compared and there is certainly a relationship between the two measures of molecular volume. However, for small molecules $\left(V_{\rho}<35 \mathrm{~cm}^{3} \mathrm{~mol}^{-1}, \rho=0.002\right.$ a.u. $)$, $\widetilde{V}$ underestimates $V_{\rho}$, and as molecules become larger $\widetilde{V}$ increasingly overestimates $V_{\rho}$. The growth of $\widetilde{V}$ relative to $V_{\rho}$ is mainly due to the nature of the second moment operator, $\hat{r}^{2}$. As the dimensions of a molecule increase, second moment expectation values increase in a quadratic fashion. Despite the differences in the behaviour of the two measures of molecular volume, good agreement was found through the equation

$$
V_{\rho}=a\left(\frac{\tilde{V}}{1 \mathrm{~cm}^{3} \mathrm{~mol}^{-1}}\right)^{b}
$$

The values of $a$ and $b$, when an isodensity contour of 0.001 a.u. is used to determine $V_{\rho}$, are $a=9.4 \pm 0.7 \mathrm{~cm}^{3} \mathrm{~mol}^{-1}$ and $b=0.331 \pm 0.012$ with $R^{2}=0.977$. For $\rho=0.002$ a.u., the values are $a=7.3 \pm 0.5 \mathrm{~cm}^{3} \mathrm{~mol}^{-1}$ and $b=0.338 \pm 0.011$ with $R^{2}=0.982$. The values of $b, b \approx \frac{1}{3}$, suggest a simpler relationship exists between $V_{\rho}$ and $\widetilde{R}$, which is the case (Figure 4.2). 


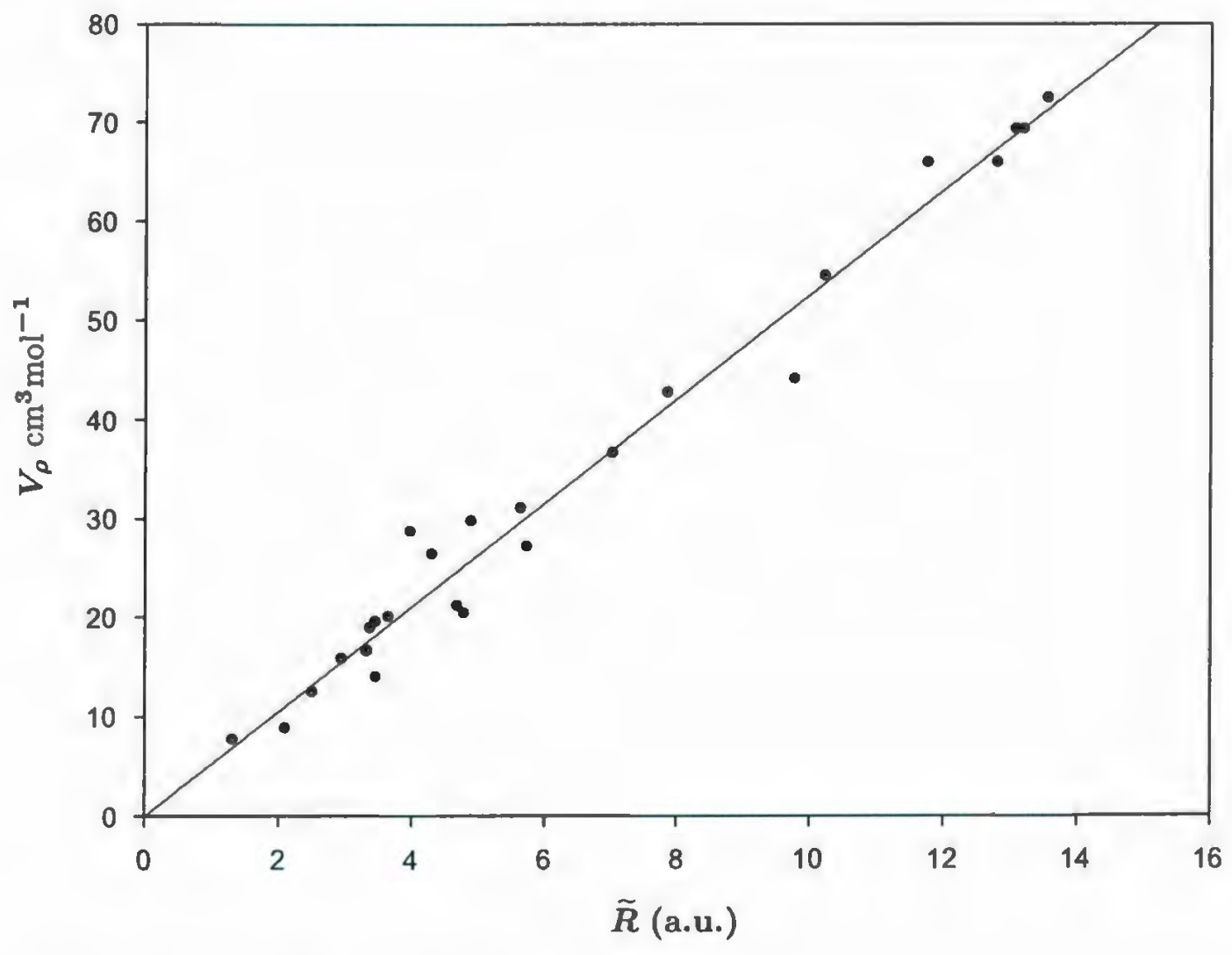

Figure 2.3. Comparison of electronic second moment average radius, $\widetilde{R}$, to Bader isodensity contour molecular volume, ${ }^{12} V_{\rho}(\rho=0.002$ a.u. $)$. 
The Bader volume of a molecule, $V_{\rho}$, is proportional to the average radius of the origin invariant electronic second moment.

$$
V_{\rho}=\alpha \widetilde{R}
$$

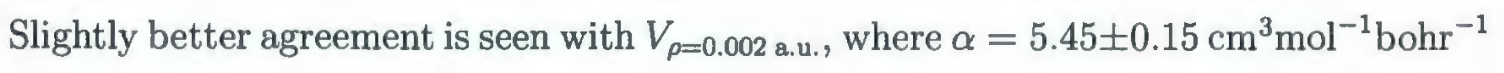

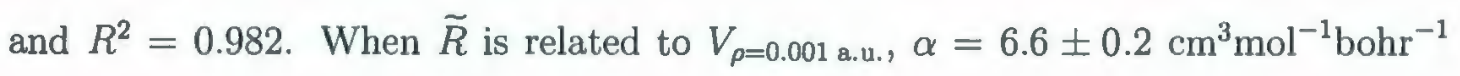
and $R^{2}=0.977$. The largest deviation from this relationship (Equation 2.17), for $\rho=0.002$ a.u. and $\rho=0.001$ a.u., is the Bader volume of ethyne. Using isodensity contours, the following relationship is observed; $V_{\rho}\left(\mathrm{CH}_{2} \mathrm{CH}_{2}\right)<V_{\rho}(\mathrm{CHCH})<$ $V_{\rho}\left(\mathrm{CH}_{3} \mathrm{CH}_{3}\right)$. However, the electronic second moment predicts $\widetilde{V}(\mathrm{CHCH})<\widetilde{V}\left(\mathrm{CH}_{2} \mathrm{CH}_{2}\right)<$ $\widetilde{V}\left(\mathrm{CH}_{3} \mathrm{CH}_{3}\right)$. Hence it appears that the two methods apply different weights to diffuse $\pi$-bond density and the density surrounding terminal hydrogens. Yet, while the two methods differ greatly in complexity of calculation, they correlate quite well via Equation 2.17. 
TABLE 2.1: Molecular shape and volume from origin invariant electronic second moment (HF/6-31G(d)//HF/6-31G(d))

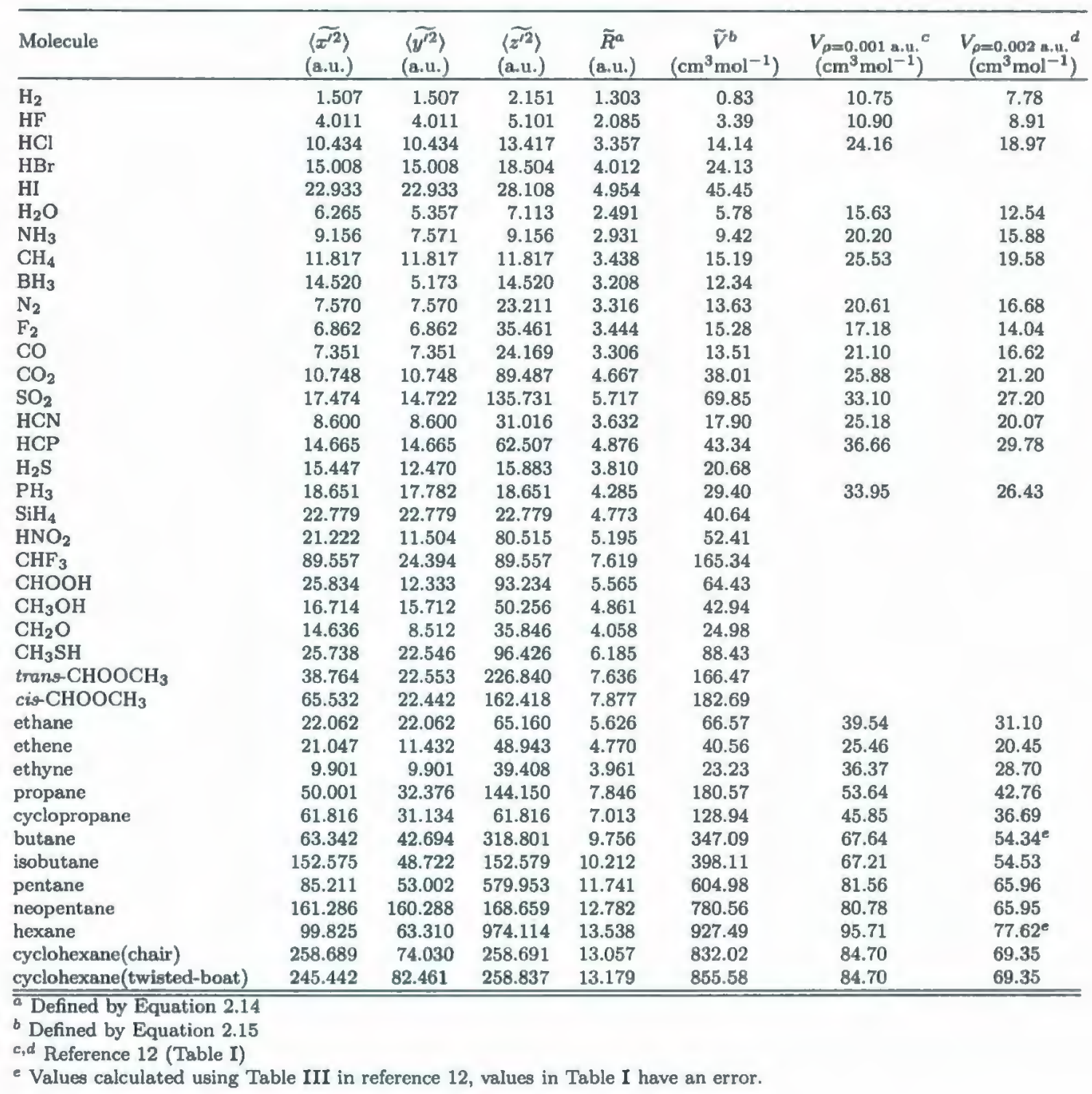




\subsection{Steric Effects}

It is evident that the origin invariant electronic second moment tensor, $\widetilde{\mathcal{S}}$, is a valid property for determining the size of a molecule. The question is now asked, can $\widetilde{\mathcal{S}}$ be used to predict the steric effects of substituents? For a substituent $X$, the electronic second moment of the corresponding $\mathrm{H} X$ molecule is used. The relationship between $\widetilde{R}$ and the Unger and Hansch modified Taft steric constant, ${ }^{3} E_{S}^{e}$, is presented in Figure 2.4. There is a relationship between the modified Taft constant, $E_{S}^{e}$, and

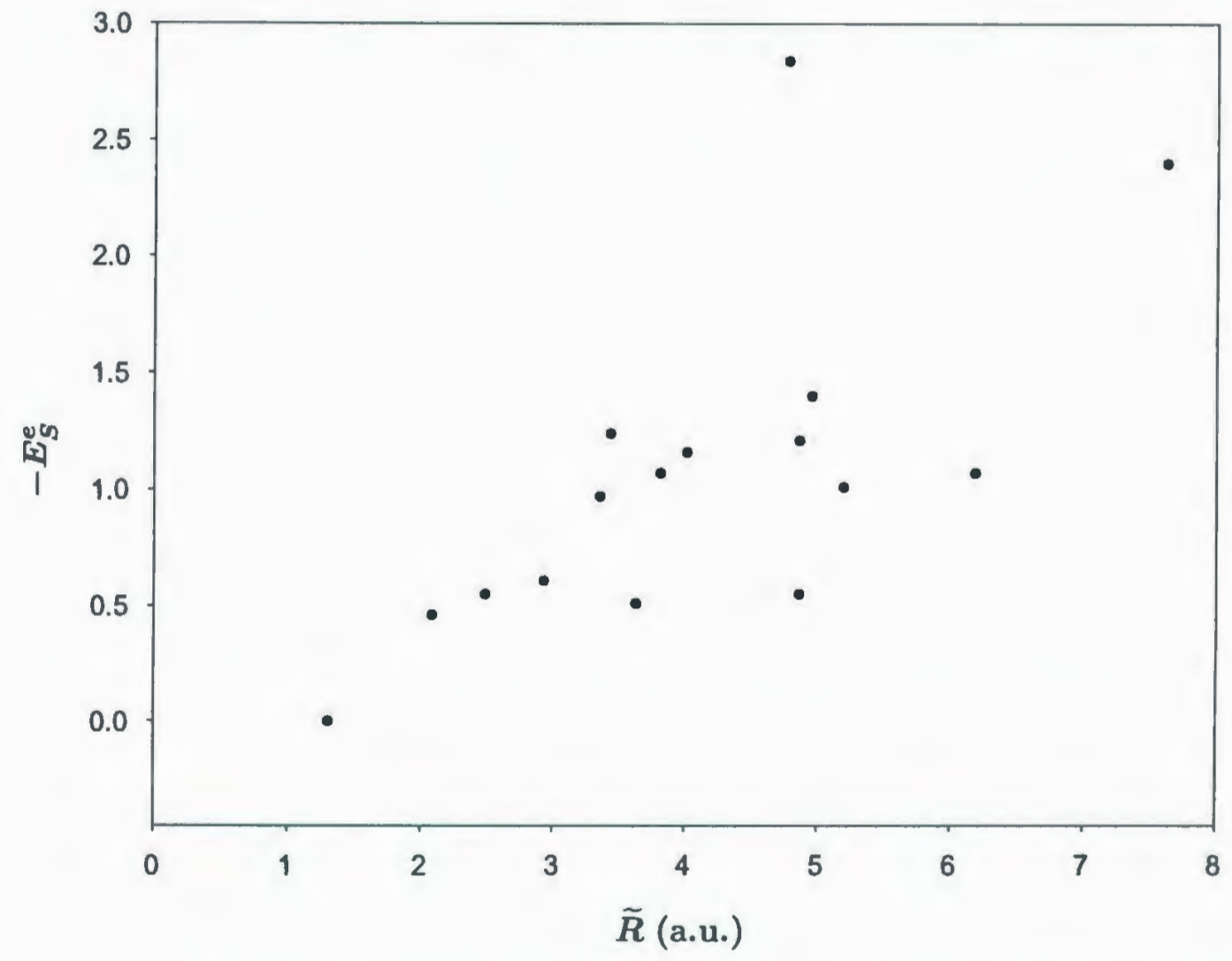

Figure 2.4. Relationship between the electronic second moment average radius, $\widetilde{R}$, and the modified Taft steric constant, $E_{S}^{e}{ }^{3}$ 
$\widetilde{R}$, but there are some significant deviations. For halogens, and first row hydrides the linear relationship is followed quite closely. However, when a substituent is of the form, $X=Y-Z$, such as $X=\mathrm{C} \equiv \mathrm{N}, \mathrm{O}-\mathrm{CH}_{3}, \mathrm{O}-\mathrm{N}-\mathrm{O}$, and $\mathrm{S}-\mathrm{CH}_{3}, \widetilde{R}$ overestimates $E_{S}^{e}$. The one significant positive deviation involves ethene, where $\widetilde{R}$ predicts a much smaller steric effect. When steric factors are measured via a chemical reaction there are several variables involved, including the conformation of the substituent during the rate determining step. It is noticed that the $E_{S}^{e}$ values for $X=\mathrm{OCH}_{3}$ and $\mathrm{OH}$ are both -0.55 , and the values for $X=\mathrm{SCH}_{3}$ and $\mathrm{SH}$ are both -1.07 . In those cases it is evident that the reaction is affected by the $\mathrm{O}$ and the $\mathrm{S}$ and not the entire substituent as measured by $\widetilde{R}$. In the case of ethene, the conformation of the vinyl substituent may directly interfere with the rate determining step resulting in a very large $E_{S}^{e}$ value. It appears that while $\widetilde{R}$ correlates moderately well with $E_{S}^{e}$, it is necessary to consider the type of substituent and how it may affect the hydrolysis reaction.

In the case of halogens, there are $P$-values available for comparison. ${ }^{7} \mathrm{~A}$ plot of $\widetilde{R}$ against the $P$-values of the halogens and hydrogen is given in Figure 2.5. As expected, $\widetilde{R}$ predicts the same trend among these substituents. However, the relationship is not quite linear and difficult to determine with few data points. There is more data available for the more extensive measure of the spatial requirement of substituents, $n$-values.

The $n$-values for various substituents, $X$, were also plotted against the average radius $\widetilde{R}$ of molecules $\mathrm{H} X$ (Figure 2.6). The $n$-values for the substituents studied correlate quite well with $\widetilde{R}, R^{2}=0.849$. The only significant deviation involves $\mathrm{NO}_{2}$, where $\widetilde{R}$ predicts a larger $n$-value than what is observed experimentally. For all other substituents, $\widetilde{R}$ predicts the same order of spatial requirement. During 


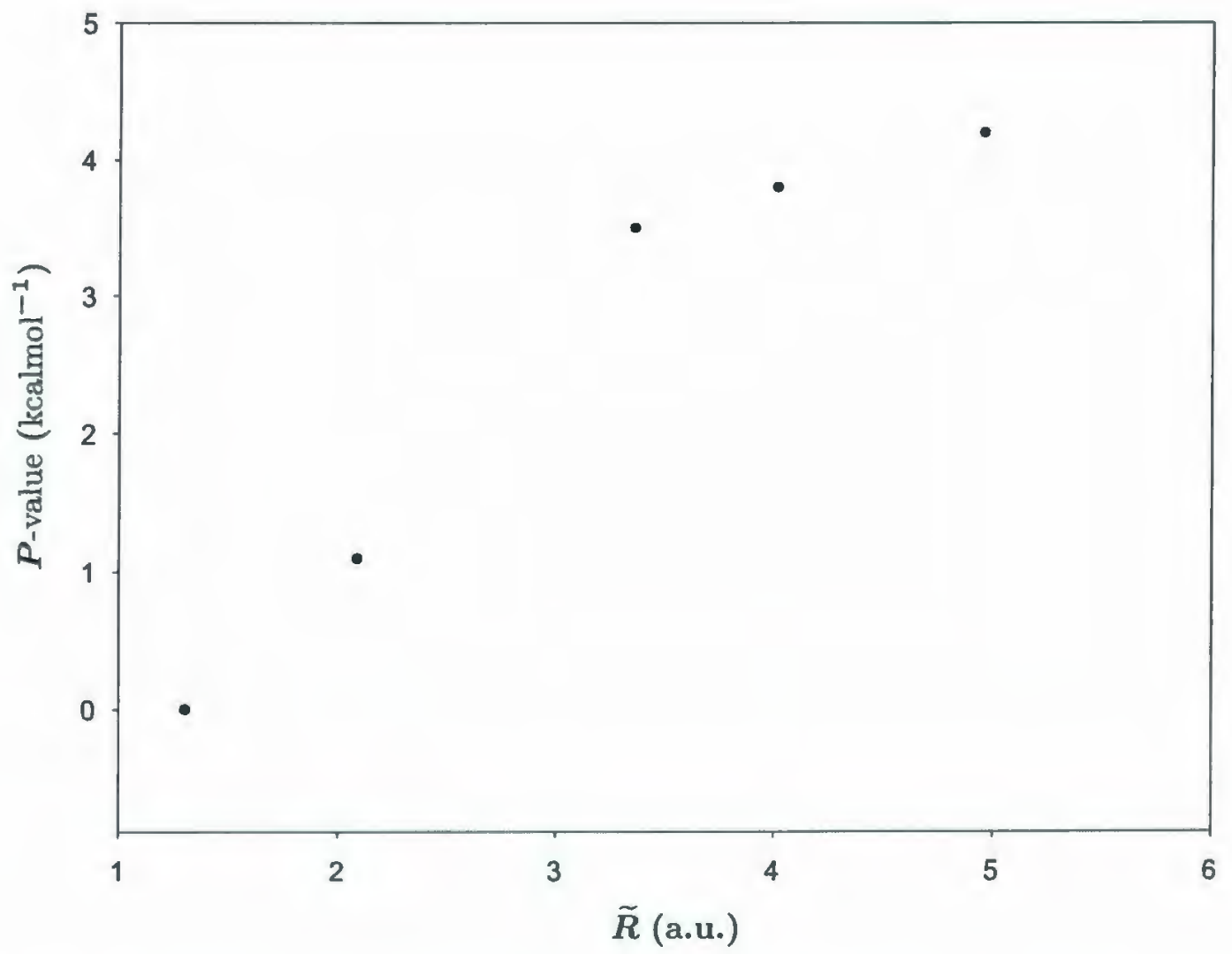

Figure 2.5. Relationship between the electronic second moment average radius, $\widetilde{R}$, and $P$-value. ${ }^{5,7}$ 


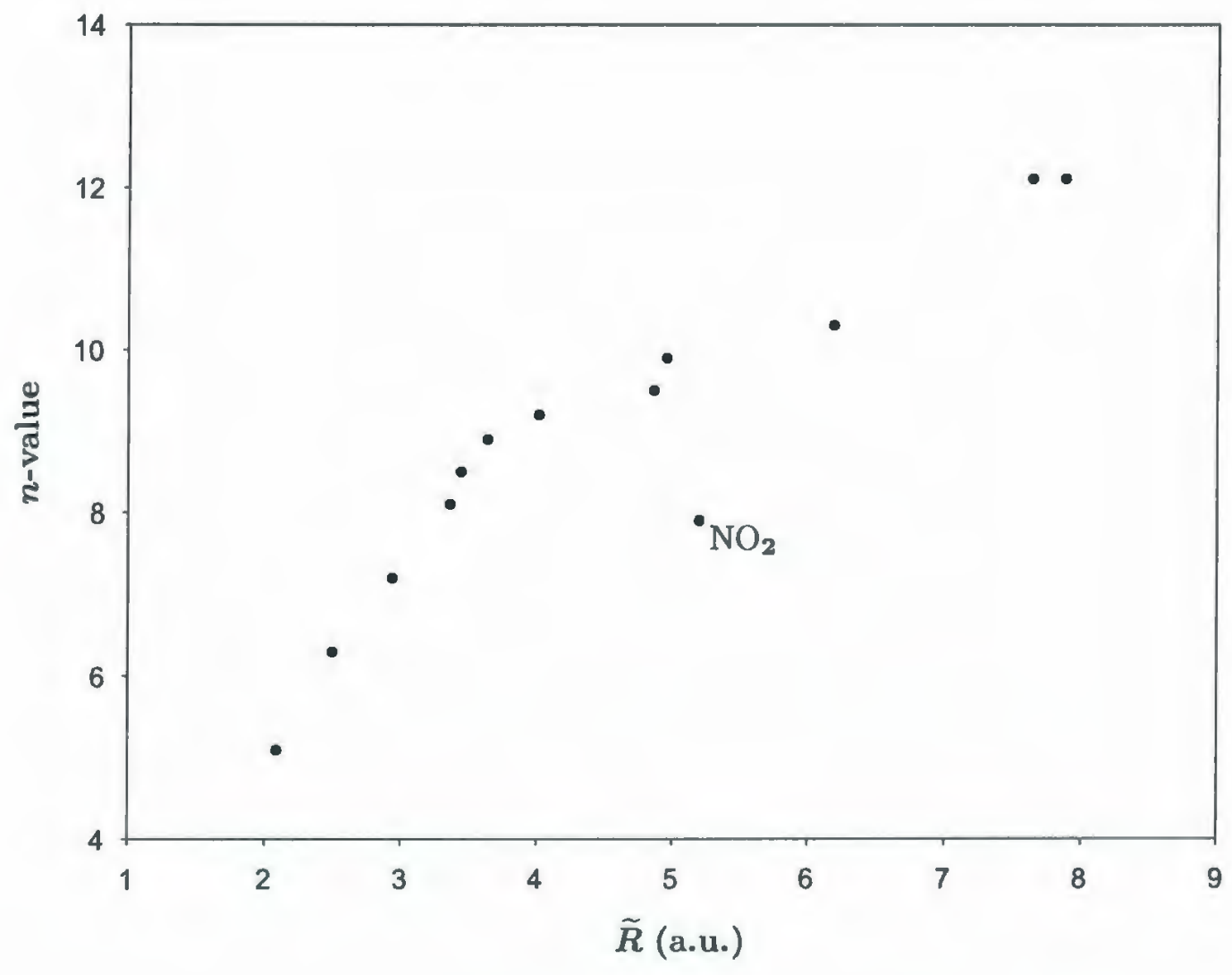

Figure 2.6. Relationship between the electronic second moment average radius, $\widetilde{R}$, and $n$-value. ${ }^{\top}$ 
a conformational process as the one described in Figure 2.1, other effects, such as electronic effects, are minimized but are still present and these effects vary among substituents. While the determination of $n$-values is quite successful with few data points and linear interpolation of $\Delta G_{c}^{\ddagger}=15 \mathrm{kcal} \mathrm{mol}^{-1}$, it is possible that $\mathrm{NO}_{2}$ interacts favourably with the aliphatic chain, resulting in a relatively lower $n$-value.

The tensor $\widetilde{S}$, and the value $\widetilde{R}$ provide purely steric information, therefore when compared with experimental measures, deviations will be observed when other effects become significant.

\section{Conclusions}

The origin invariant electronic second moment tensor, $\widetilde{\mathcal{S}}$, is a valid measure of molecular size and consequently the spatial requirement of a substituent. There are several pre-existing methods for determining such values, both experimentally and computationally. The shape and size of a molecule as defined here correlates well with many of these pre-existing methods and is relatively easy to compute. The tensor consists of one electron expectation values which require far less complex and extensive calculations than existing quantum mechanical approaches. In most cases, the square root of the geometric average of the eigenvalues of $\widetilde{\mathcal{S}}, \widetilde{R}$, can predict the steric effects of substituents as described by the modified Taft $E_{S}^{e}, P$-values, and $n$-values. While there are some deviations, consideration of the substituents being compared may lead to better estimates. Also, the shape as described by the principal axes of the second moment tensor, $\left.\left\langle\widetilde{x^{\prime 2}}\right\rangle, \widetilde{\left\langle y^{\prime 2}\right.}\right\rangle$ and $\left\langle\widetilde{z^{\prime 2}}\right\rangle$, could be applied specifically when certain interactions are expected in a chemical reaction or conformational process. 


\section{References}

(1) R. W. Taft. J. Am. Chem. Soc., 74:3120-3128, 1952.

(2) M. S. Newman. Steric Effects in Organic Chemistry. Wiley, first edition, 1956.

(3) S. H. Unger and C. Hansch. Prog. Phys. Org. Chem., 12:91-118, 1976.

(4) J. A. MacPhee, A. Panaye, and J.-E. Dubois. Tetrahedron Lett., pages 32933296, 1978.

(5) J. E. Anderson and H. Pearson. J. Chem. Soc. Chem. Comm., pages 871-872, 1971.

(6) S. Winstein and N. J. Holness. J. Am. Chem. Soc., 77:5562-5578, 1955.

(7) H. Förster and F. Vögtle. Angew. Chem. Int. Ed. Engl., 16:429-441, 1977.

(8) A. Bondi. J. Phys. Chem., 68:441-451, 1964.

(9) J. C. Slater. J. Chem. Phys., 41:3199-3204, 1964.

(10) A. Gavezzotti. J. Am. Chem. Soc., 105:5220-5225, 1983.

(11) R. F. W. Bader, W. H. Henneker, and P. E. Cade. J. Chem. Phys., 46:3341-3363, 1967.

(12) R. F. W. Bader, M. T. Carrol, J. R. Cheeseman, and C. Chang. J. Am. Chem. Soc., 109:7968-7979, 1987.

(13) M. A. Robb, W. J. Haines, and I. G. Csizmadia. J. Am. Chem. Soc., 95:42-48, 1973. 
(14) I. G. Csizmadia. Localization and Delocalization in Quantum Chemistry, 1:349353, 1975.

(15) R. A. Poirier and J. W. Hollett. MUNgauss (Fortran 90 version). Chemistry Department, Memorial University of Newfoundland, St. John's, NL, A1B 3X7. With contributions from S. D. Bungay, A. El-Sherbiny, T. Gosse, D. Keefe, A. Kelly, C. C. Pye, D. Reid, K. Saputantri, M. Shaw, M.S. Staveley, Y. Wang and J. Xidos.

(16) J. Andzelm, M. Klobukowski, E. Radzio-Andzelm, Y. Sakai, and H. Tatewaki. Physical Sciences Data 16 Gaussian Basis Sets for Molecular Calculations. Elsevier Science Publishers, 1984.

(17) Gaussian 03, revision a.9. Gaussian Inc.; Pittsburgh, PA, 2003. 
The following Chapter, Chapter 3, is published as an article in the Journal of Theoretical and Computational Chemistry.

J. W. Hollett and R. A. Poirier, J. Theoretical Computational Chem., 6:13-22, 2007.

J. W. Hollett is the principal author and performed all the research under the supervision of R. A. Poirier. 


\section{Chapter 3}

\section{An interesting relationship}

\section{between interelectronic distance}

and the corresponding Coulomb

\section{integral}

1 Introduction

In wave function theory, the electronic energy, $E$, is given as an expectation value (3.1),

$$
E=\langle\Psi|\hat{H}| \Psi\rangle
$$

where $\Psi$ is the wave function, with $\langle\Psi \mid \Psi\rangle=1$, and $\hat{H}$ is the electronic Hamiltonian, in atomic units (3.2).

$$
\hat{H}=-\sum_{i=1}^{N} \frac{1}{2} \nabla_{i}^{2}-\sum_{i=1}^{N} \sum_{A=1}^{M} \frac{Z_{A}}{r_{i A}}+\sum_{i=1}^{N} \sum_{j>i}^{N} \frac{1}{r_{i j}}
$$


The electronic Hamiltonian consists of a kinetic energy operator, a nuclear attraction potential energy operator, and an electron-electron potential energy operator, $\sum_{i=1}^{N} \sum_{j>i}^{N} \frac{1}{r_{i j}}$. For the Hartree-Fock wave function, a single Slater determinant, the energy, $E_{H F}$, of the system is given as,

$$
E_{H F}=2 \sum_{a=1}^{N / 2} h_{a a}+\sum_{a=1}^{N / 2} \sum_{b=1}^{N / 2} 2 J_{a b}-K_{a b}
$$

The above expression is for a closed shell system, $N$ electrons occupying $N / 2$ molecular orbitals. The $h_{a a}$ terms are one-electron integrals representing the kinetic and nuclear attraction potential energy. The $J_{a b}$ and $K_{a b}$ terms are the two-electron Coulomb and exchange integrals, respectively, for an electron in molecular orbital $a$, and an electron in molecular orbital $b$. The Coulomb integral, $J_{a b}$, is of particular importance to this discussion and it is given as,

$$
J_{a b}=\iint \psi_{a}^{*}\left(r_{1}\right) \psi_{b}^{*}\left(r_{2}\right) \frac{1}{r_{12}} \psi_{a}\left(r_{1}\right) \psi_{b}\left(r_{2}\right) d r_{1} d r_{2}=\langle a b \mid a b\rangle
$$

where $\psi_{a}$ and $\psi_{b}$ are molecular orbitals (spatial orbitals), $r_{1}$ and $r_{2}$ are the position vectors of the two electrons, and $r_{12}$ is the distance between the two electrons. The distance between two electrons is a quantity that does not appear in the wave function itself. This leads to the drawback of Hartree-Fock theory, the failure to account for electron correlation. There are several theories that attempt to account for electron correlation such as configuration interaction and perturbation theory, which begin with the Hartree-Fock wave function. However, an alternative approach is the use of a wave function constructed from two-electron functions.

In most theories, two-electron wave functions are created by the addition, or multiplication, of a single $r_{12}$ term. In 1929, Hylleraas constructed a correlated wave 
function, with an $r_{12}$ term, for the calculation of the energy of the helium atom. ${ }^{1}$ In 1948 , Frost et al. used a binomial term of the form $\left(1+p r_{12}\right)$, where $p$ is a constant, to construct correlated molecular orbitals. ${ }^{2}$ There is also a form of MP2 theory, MP2R12, which incorporates an $r_{12}$ term into the unperturbed wave function which is expanded in partial waves. ${ }^{3}$ Unfortunately, the disadvantage to using two-electron functions is the complications they cause in the calculation of energies and other properties of molecules. At this time, a practical implementation of these "correlated methods" has not been developed despite various attempts.

Accounting for electron correlation is the main focus of most applications of the "interelectronic" ${ }^{2}$ distance. However, construction of a two-electron wave function may not be the only route. The application of an "interelectronic" distance, calculated from one-electron expectation values, to the electron correlation problem may also be a possibility.

Relating one-electron properties of a wave function to the energy of the system is not a new idea. There have been various investigations into the relationship between the second moment and different energy components of atoms and molecules. ${ }^{4-8}$ The second moment, $\left\langle r^{2}\right\rangle$, of a system is related to its "size". ${ }^{9}$ A theoretical definition of the "size" of an electron pair associated with a Localized MO (LMO), $\left\langle r_{1}^{2}\right\rangle_{R_{a}}$, is given as $(3.5),{ }^{9}$

$$
\left\langle r_{1}^{2}\right\rangle_{R_{a}}=\left|\left\langle\psi_{a}\left|r_{1}^{2}\right| \psi_{a}\right\rangle_{0}-R_{a}^{2}\right|
$$

where $R_{a}$ is the centroid of charge of the LMO,

$$
\left|R_{a}\right|=\sqrt{\left\langle\psi_{a}\left|x_{1}\right| \psi_{a}\right\rangle_{0}^{2}+\left\langle\psi_{a}\left|y_{1}\right| \psi_{a}\right\rangle_{0}^{2}+\left\langle\psi_{a}\left|z_{1}\right| \psi_{a}\right\rangle_{0}^{2}}
$$

calculated at some point $R_{0}$. 
Through various ${ }^{4-6}$ studies, the following relationship was found between the orbital energy, $\epsilon_{i}$, of a LMO and its size,

$$
\left(\frac{1}{\epsilon_{i}}\right)=m\left\langle r_{i}^{2}\right\rangle+\left(\frac{1}{\epsilon_{i}}\right)_{0}
$$

where $m$ is a fitted constant. ${ }^{6}$ Although core LMOs followed the above relationship, there are significant deviations among the bonding and valence LMOs. ${ }^{6}$ The correlation energy, $E_{\text {corr }}$, for atoms and ions was found to be related to the total second moment by the equation

$$
\left(\left|E_{c o r r}\right|\left\langle r^{2}\right\rangle\right)^{r}=k(Z-\sigma)
$$

where $Z$ is the nuclear charge and $\gamma, \sigma$, and $k$ are constants optimized for each isoelectronic series of ions. ${ }^{7}$ Another relationship involving the size of individual LMOs, $\left\langle r^{2}\right\rangle_{i}$, and the correlation energy was also found, $(3.9){ }^{8}$

$$
E_{\text {orr }}=-0.06593 \sum_{i=1}^{M}\left\langle r^{2}\right\rangle_{i}^{-0.1958}
$$

The concept of size applies to MOs and entire systems, yet it is well known that correlation occurs between electrons occupying different MOs. This is an instance in which the concept of size has no meaning. However, distance applies to all pairs of electrons. This is, in part, why we propose the idea of the distance between two electrons, and also because of the simplicity of its calculation.

\section{Theory}

\subsection{The Distance Between Two Electrons, $\left(\delta r_{12}\right)_{a b}$}

Given the Cartesian coordinates of two electrons, $r_{1}=\left(x_{1}, y_{1}, z_{1}\right)$ and $r_{2}=$ $\left(x_{2}, y_{2}, z_{2}\right)$, the distance between the two, $r_{12}$, is given as

$$
r_{12}=\sqrt{\left(x_{1}-x_{2}\right)^{2}+\left(y_{1}-y_{2}\right)^{2}+\left(z_{1}-z_{2}\right)^{2}}
$$


The above expression can then be expanded to give

$$
r_{12}=\sqrt{x_{1}^{2}+x_{2}^{2}-2 x_{1} x_{2}+y_{1}^{2}+y_{2}^{2}-2 y_{1} y_{2}+z_{1}^{2}+z_{2}^{2}-2 z_{1} z_{2}}
$$

Now consider two electrons, one occupying molecular orbital $a$, and another occupying molecular orbital $b$. We define the distance between these two electrons by substituting expectation values of molecular orbitals $a$ and $b$ for the corresponding Cartesian coordinate values in equation (3.11). These expectation values have the form;

$$
\int \psi_{a}^{*}\left(r_{1}\right) i^{k} \psi_{a}\left(r_{1}\right) d r_{1}=\left\langle a\left|i^{k}\right| a\right\rangle=\left\langle i^{k}\right\rangle_{a}
$$

where $i=x, y$, or $z$, and $k=1$ or 2 , and the distance between an electron in molecular orbital $a$ and an electron in molecular orbital $b,\left(\delta r_{12}\right)_{a b}$, is given as,

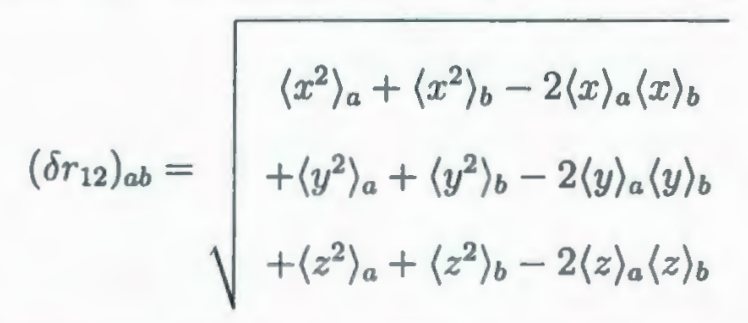

For two electrons occupying the same molecular orbital $(a=b)$, equation (3.13) reduces to

$$
\left(\delta r_{12}\right)_{a b}=\sqrt{\begin{array}{c}
2\left\langle x^{2}\right\rangle_{a}-2\langle x\rangle_{a}^{2} \\
+2\left\langle y^{2}\right\rangle_{a}-2\langle y\rangle_{a}^{2} \\
+2\left\langle z^{2}\right\rangle_{a}-2\langle z\rangle_{a}^{2}
\end{array}}=\sqrt{2} \sqrt{\left\langle r^{2}\right\rangle_{a}-\langle r\rangle_{a}^{2}},
$$

which is the root mean square deviation of the position of an electron in molecular orbital $a$, multiplied by $\sqrt{2}$. It should also be noted that the distance between two electrons, as we have defined, is invariant with respect to the selected origin. Consider the origin to be some point $r_{0}=\left(x_{0}, y_{0}, z_{0}\right)$, then $\left(\delta r_{12}\right)_{a b}$ becomes

$$
\left(\delta r_{12}\right)_{a b}=\sqrt{\left\langle\left(x+x_{0}\right)^{2}\right\rangle_{a}+\left\langle\left(x+x_{0}\right)^{2}\right\rangle_{b}-2\left\langle\left(x+x_{0}\right)\right\rangle_{a}\left\langle\left(x+x_{0}\right)\right\rangle_{b}+\ldots}
$$




$$
\begin{gathered}
\left(\delta r_{12}\right)_{a b}=\sqrt{\left\langle x^{2}+x_{0}^{2}+2 x x_{0}\right\rangle_{a}+\left\langle x^{2}+x_{0}^{2}+2 x x_{0}\right\rangle_{b}-2\left(\langle x\rangle_{a}+x_{0}\right)\left(\langle x\rangle_{b}+x_{0}\right)+\ldots} \\
\left(\delta r_{12}\right)_{a b}=\sqrt{\begin{array}{l}
\left\langle x^{2}\right\rangle_{a}+\left\langle x^{2}\right\rangle_{b}+2 x_{0}\langle x\rangle_{a}+2 x_{0}\langle x\rangle_{b}+2 x_{0}^{2} \\
-2\langle x\rangle_{a}\langle x\rangle_{b}-2 x_{0}\langle x\rangle_{a}-2 x_{0}\langle x\rangle_{b}-2 x_{0}^{2}+\ldots
\end{array}} \\
\left(\delta r_{12}\right)_{a b}=\sqrt{\left\langle x^{2}\right\rangle_{a}+\left\langle x^{2}\right\rangle_{b}-2\langle x\rangle_{a}\langle x\rangle_{b}+\ldots}
\end{gathered}
$$

\section{Method}

All calculations were performed using the MUNgauss program. ${ }^{10}$ For the twoelectron systems studied, singlet calculations were performed at the restricted HartreeFock(RHF) level of theory, while unrestricted Hartree-Fock(UHF) theory was used for the triplet calculations. Both the triplet and singlet calculations, for $\mathrm{He}$ to $\mathrm{Ne}^{8+}$ were performed using the 6-31G basis set and a 10s uncontracted Huzinaga basis set(H10s), ${ }^{11}$ which is optimized for $\mathrm{He}$. For He, singlet and triplet calculations were also performed with the 6-311G basis set. For all the systems with more than two electrons, calculations were performed at RHF/6-31G(d)//RHF/6-31G(d). In the case of the four and ten electron systems, calculations were also performed using LMOs, which were obtained via the Boys localization method. ${ }^{12}$

\section{Results and Discussion}

\subsection{Behaviour of $\left(\delta r_{12}\right)_{a b}$}

The behaviour of $\left(\delta r_{12}\right)_{a b}$ is demonstrated by the values calculated for a series of two-electron species, from $\mathrm{He}$ to $\mathrm{Ne}^{8+}$, both in the singlet and triplet states. Plots of the singlet and triplet data are given in Figure 3.1 and Figure 3.2 respectively. For the singlet systems the distance between the two electrons decreases smoothly with 
increasing atomic charge, which is what would be expected intuitively. Also, there is no significant difference between the $\left(\delta r_{12}\right)_{a b}$ values calculated with different basis sets. Although it should be noted that there is a slight decrease in all $\left(\delta r_{12}\right)_{a b}$ values when the larger H10s basis set is used. It should also be noted that in the case of two electrons occupying the same $\mathrm{MO},\left(\delta r_{12}\right)_{a a}$ is equivalent to the square root of the size, defined by Csizmadia et. al., ${ }^{9}$ multiplied by $\sqrt{2}$. For the triplet systems, where the

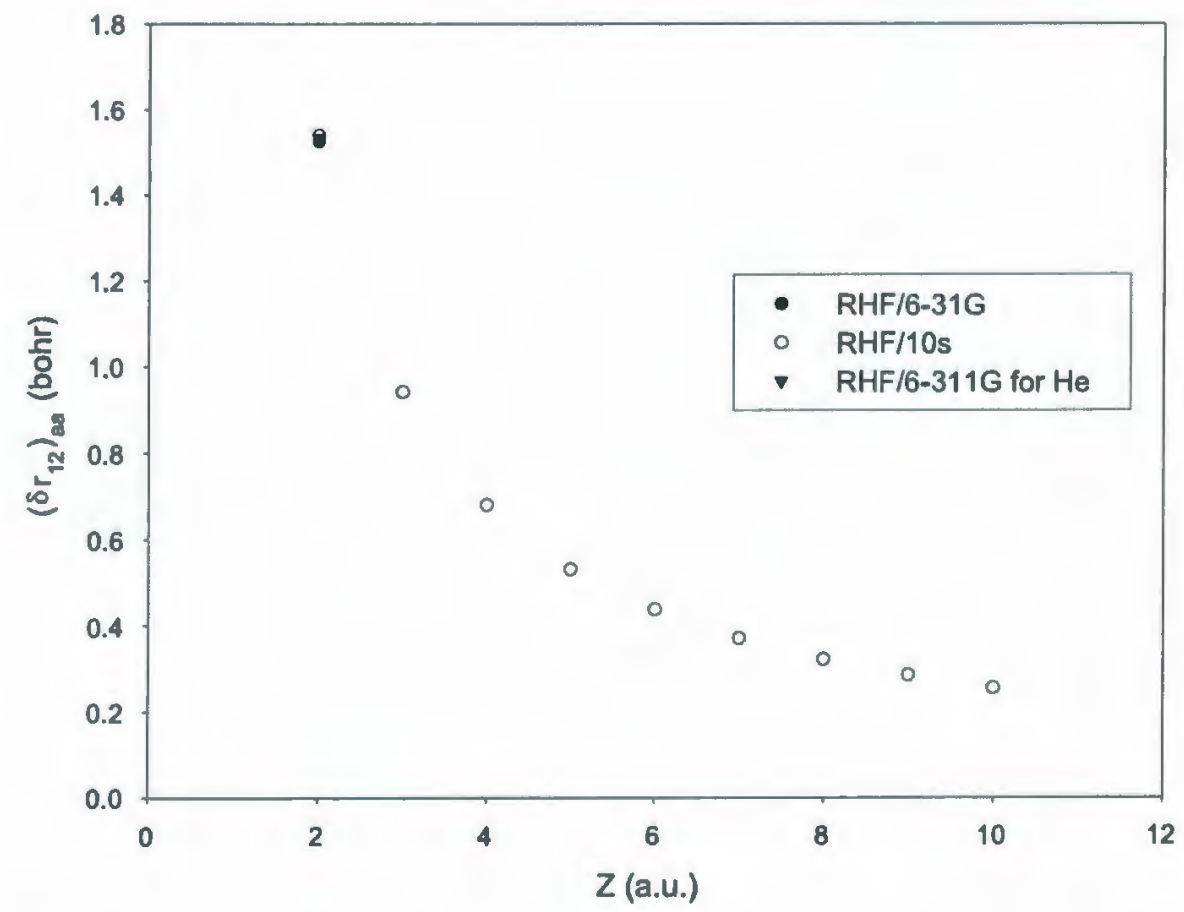

Figure 3.1. $\left(\delta r_{12}\right)_{a b}$ of two-electron singlet systems with varying nuclear charge.

electrons now occupy two different orbitals, the $\left(\delta r_{12}\right)_{a b}$ values behave similarly to the singlet systems (Figure 3.2), but are much more sensitive to basis set. At UHF/6-31G triplet He has an $\left(\delta r_{12}\right)_{a b}$ value of $1.942 \mathrm{bohr}$, which is much lower than the trend predicts (Figure 3.2). This is most likely due to the inadequacy of the 6-31G basis set 


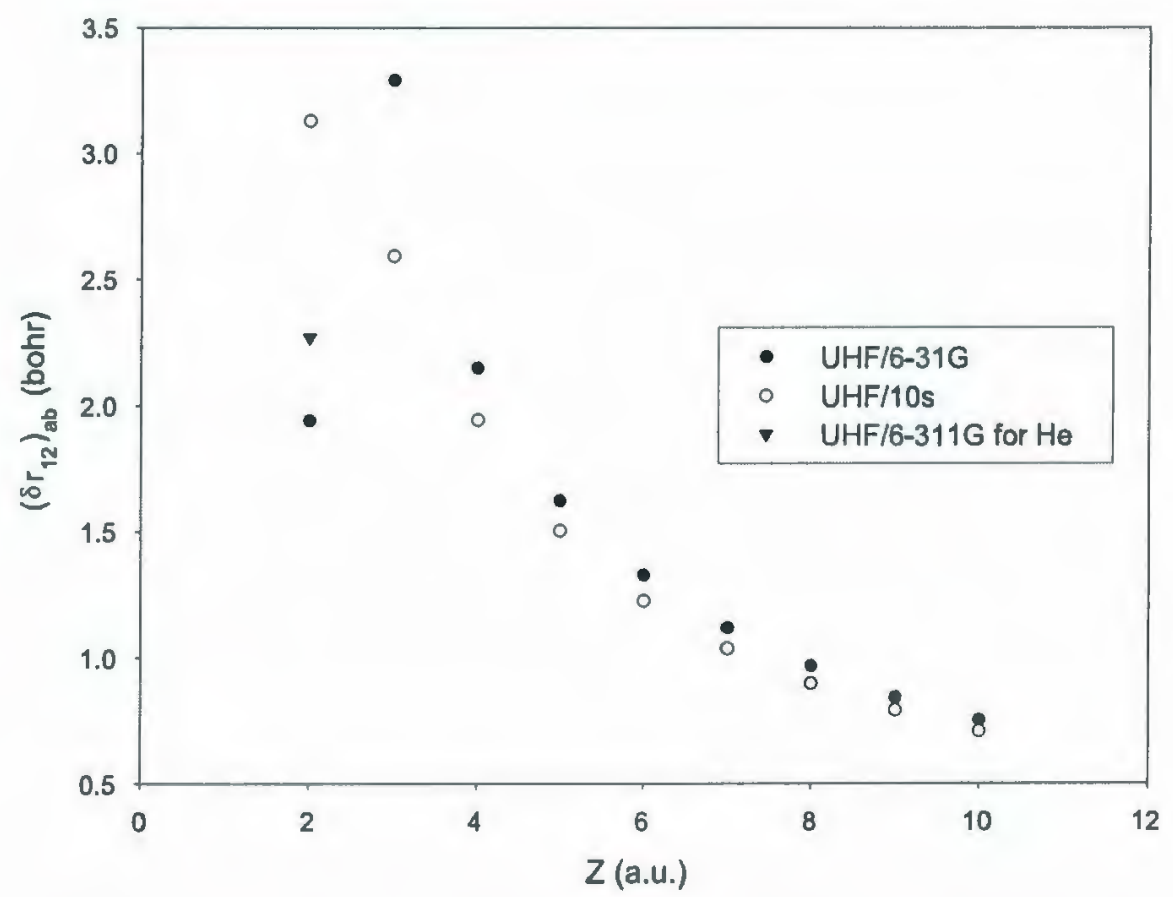

Figure 3.2. $\left(\delta r_{12}\right)_{a b}$ of two-electron triplet systems with varying nuclear charge. 
for He, which has only two s-type functions, as compared to the first row elements, which have three s-type functions. The He $\left(\delta r_{12}\right)_{a b}$ value at UHF/6-311G, $2.269 \mathrm{bohr}$, is significantly larger than the UHF/6-31G value albeit still quite far from the trend. For the large 10s basis set, the $\left(\delta r_{12}\right)_{a b}$ values are consistently lower than the 6-31G values, with the exception of He. The $\left(\delta r_{12}\right)_{a b}$ value for He with the H10s basis set is only slightly lower than the trend predicts, which is likely due to the fact that the basis set is optimized for singlet He.

The general behaviour of the distance between two electrons, as given by $\left(\delta r_{12}\right)_{a b}$, is what would be expected from an intuitive standpoint. It should also be noted that

as expected $\left(\delta r_{12}\right)_{a b}^{\text {triplet }}>\left(\delta r_{12}\right)_{a b}^{\text {singlet }}$, and for triplet systems, the value of $\left(\delta r_{12}\right)_{a b}$ is quite dependent on basis set. The behaviour of $\left(\delta r_{12}\right)_{a b}$ suggests that some relationship should also exist between $\left(\delta r_{12}\right)_{a b}$ and the corresponding Coulomb integral, $J_{a b}$. However, the relationship is found to be surprisingly good.

\subsection{Relationship with the two-electron Coulomb integral, $J_{a b}$}

The two-electron Coulomb integral, defined by equation (5.12), contains the electronelectron potential operator, $\frac{1}{r_{12}}$, and if thought of in the classical sense, involving two point charges, a relationship with the distance between two electrons is expected to some extent. As illustrated in Figure 3.3, the somewhat complex two-electron Coulomb integral has a direct linear relationship with the rather simple $\left(\delta r_{12}\right)_{a b}$ value, which is constructed of one-electron, dipole and second moment operators. The relationship is given by the equation $J_{a b}=\frac{\alpha}{\left(\delta r_{12}\right)_{a b}}$, which has the expected limits, $\left(\delta r_{12}\right)_{a b} \rightarrow \infty \Rightarrow J_{a b} \rightarrow 0$ and $\left(\delta r_{12}\right)_{a b} \rightarrow 0 \Rightarrow J_{a b} \rightarrow \infty$. For the two-electron singlet systems studied, $\mathrm{He}$ to $\mathrm{Ne}^{8+}, \alpha=1.53 \pm 0.01$ bohr hartrees with both the 6-31G 


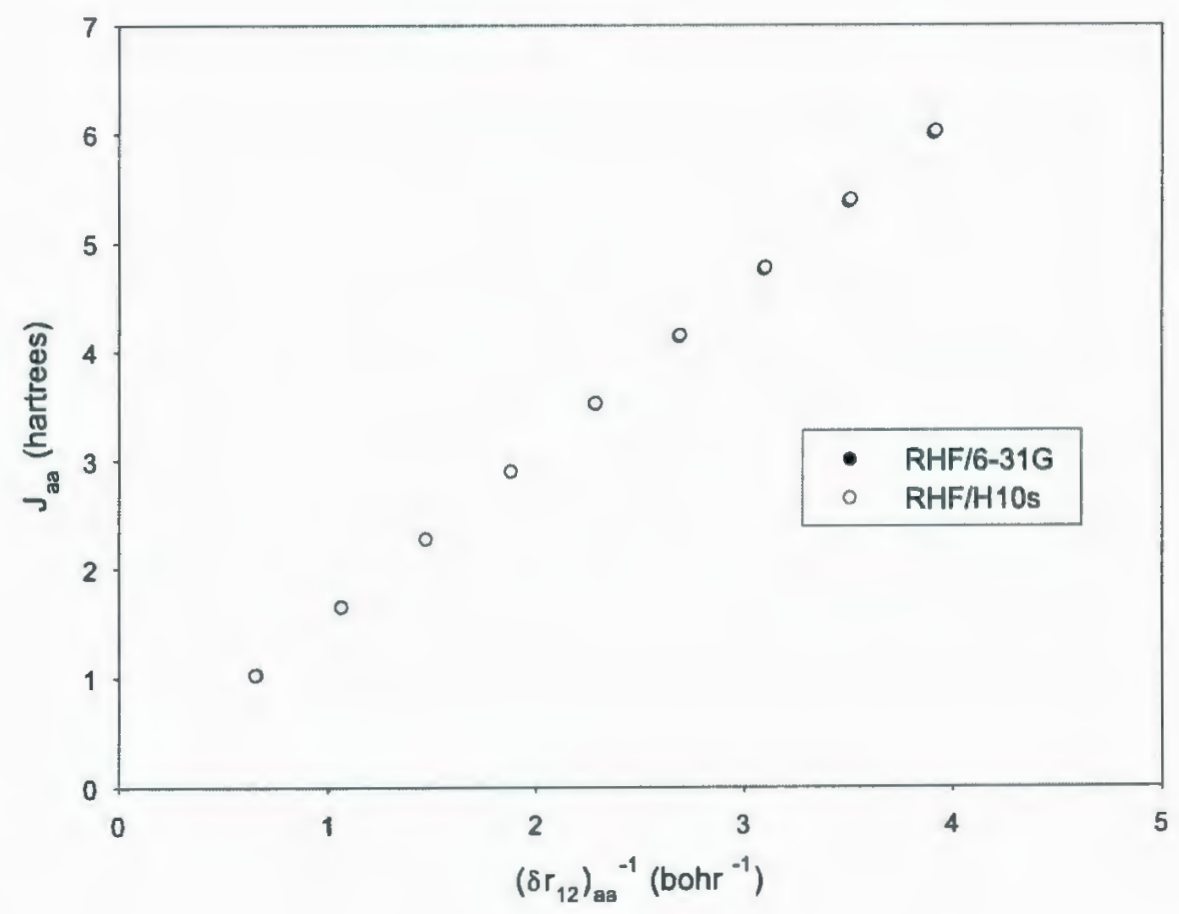

Figure 3.3. Relationship between $\left(\delta r_{12}\right)_{a a}$ and Coulomb integral, $J_{a a}$, for the singlet state of He to $\mathrm{Ne}^{8+}$. 
basis set and the H10s basis set, with $R^{2}$ values of 0.9997 and 0.9996 respectively. For the more basis set sensitive triplet systems, $\alpha=4.1 \pm 0.2$ bohr hartrees with the 6-31G basis set and $\alpha=4.12 \pm 0.07$ bohr hartrees with the H10s basis set. The corresponding $R^{2}$ values are 0.9907 and 0.9981 . The only major deviations from the relationship, for the two-electron systems, are the values corresponding to triplet $\mathrm{He}$ (Figure 3.4), which is due to the inadequacy of the 6-31G basis set, and the H10s basis set which is specifically optimized for He. Although the $\alpha$ values for the singlet and triplet states are quite different, the relationship is followed quite closely, as indicated by the $R^{2}$ values. This linear relationship extends beyond two-electron atoms and ions, and pairs of electrons in the same MO.

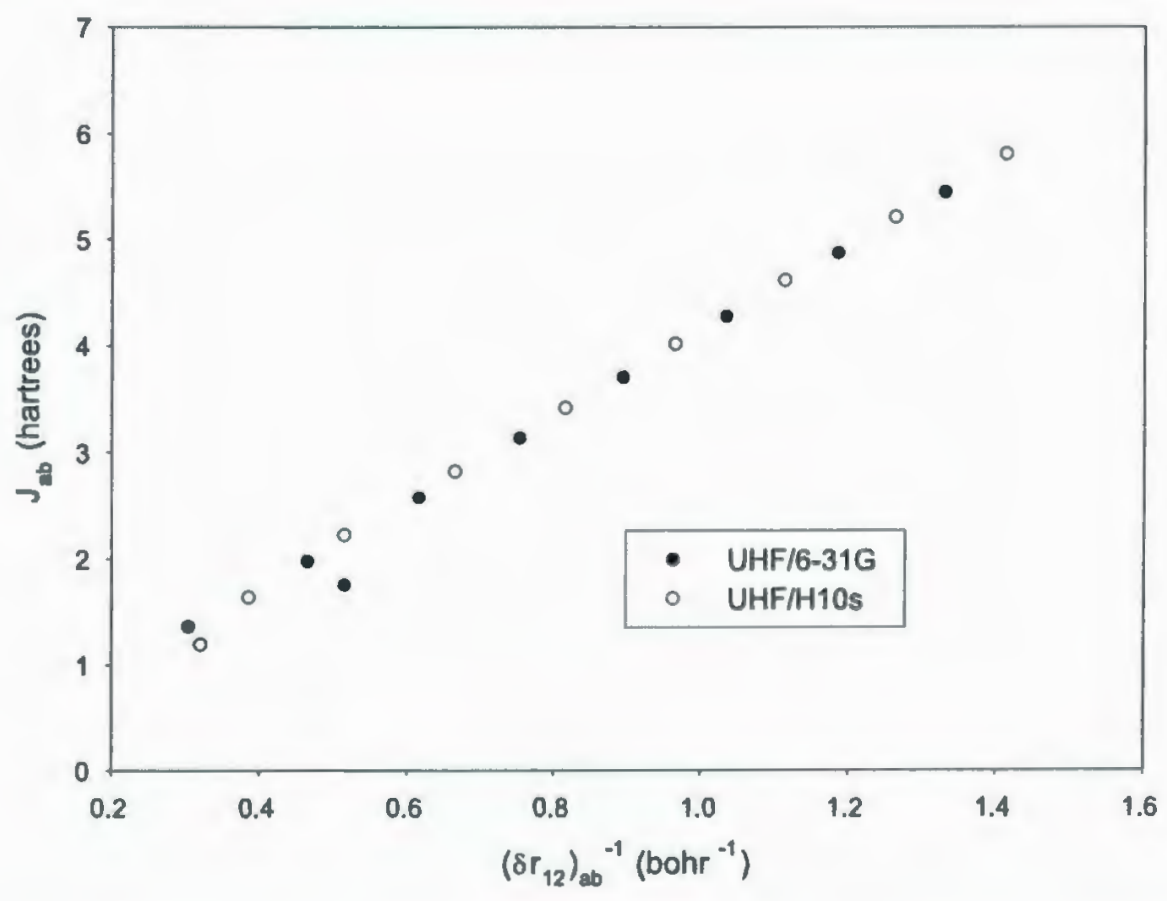

Figure 3.4. Relationship between $\left(\delta r_{12}\right)_{a b}$ and Coulomb integral, $J_{a b}$, for the triplet state of He to $\mathrm{Ne}^{8+}$. 
Systems of up to 58 electrons (uracil) were studied, and the same relationship was found between the distance between two electrons in given MOs, $\left(\delta r_{12}\right)_{a b}$, and the corresponding Coulomb integral, $J_{a b}$, regardless of whether the electrons occupy the same MO or not. The relationship in four-electron systems $(\mathrm{Be}, \mathrm{LiH})$ and tenelectron systems $\left(\mathrm{Ne}, \mathrm{HF}, \mathrm{H}_{2} \mathrm{O}, \mathrm{NH}_{3}\right.$, and $\left.\mathrm{CH}_{4}\right)$ is illustrated in Figure 3.5. The values of $\left(\delta r_{12}\right)_{a b}$ and $J_{a b}$ calculated using LMOs are also included in this plot, which illustrates the existence of the relationship $J_{a b}=\frac{\alpha}{\left(\delta r_{12}\right)_{a b}}$ for both canonical and localized molecular orbitals. For CMOs the value of $\alpha$ is $1.56 \pm 0.01$ bohr hartrees, with

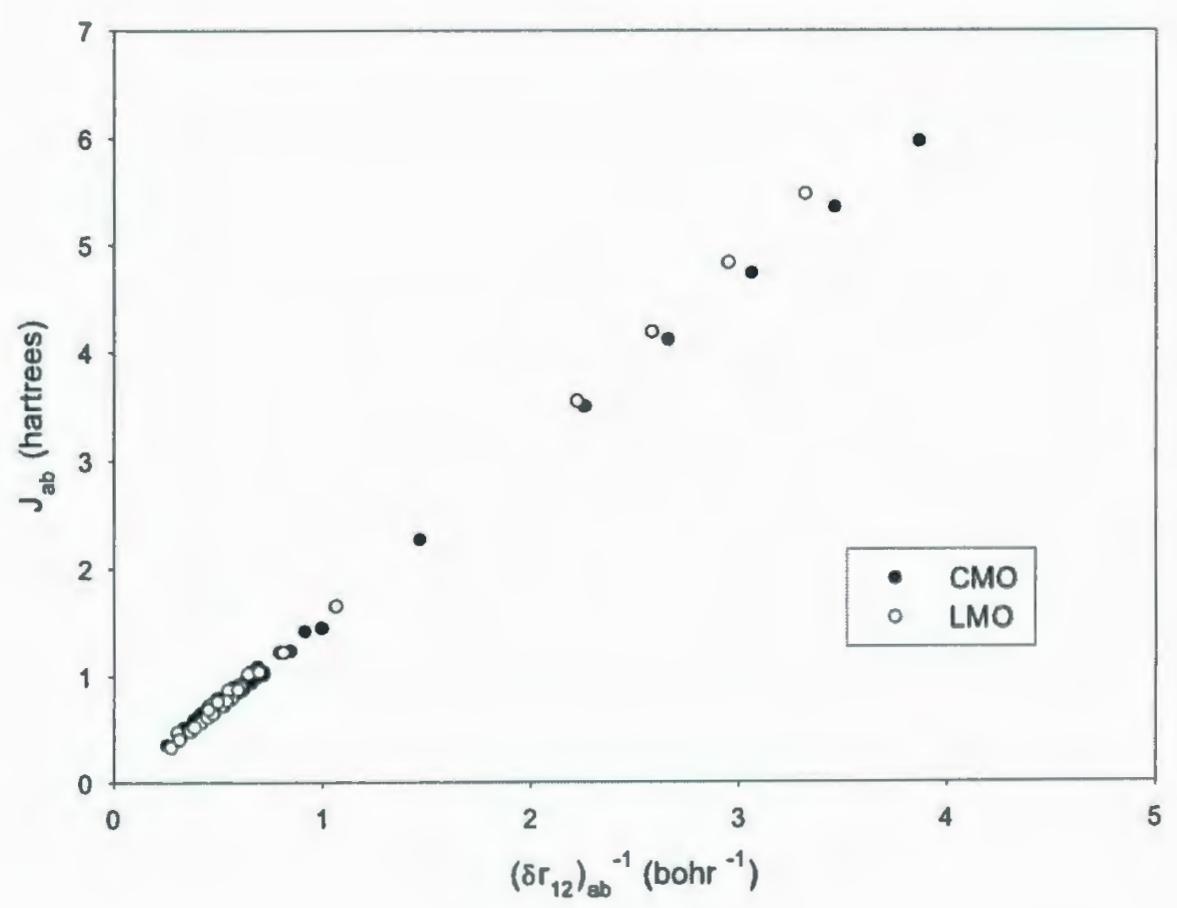

Figure 3.5. Relationship between $\left(\delta r_{12}\right)_{a b}$ and corresponding Coulomb integral, $J_{a b}$, for systems of four and ten electrons, calculated at HF/6-31G(d) using CMOs and LMOs.

$R^{2}=0.9988$. When LMO values are used there is a slight difference in the value of 
$\alpha, \alpha=1.669 \pm 0.024$ bohr hartrees, with similar correlation, $R^{2}=0.9869$. It is seen that there is little deviation from the relationship for these atoms and single heavy atom hydrides (Figure 3.5). However for larger molecules, such as carbon dioxide, propane and uracil, there is more deviation. The relationship between $\left(\delta r_{12}\right)_{a b}$ and $J_{a b}$, for all the systems (except the triplet systems), is shown in Figure 3.6. The linear

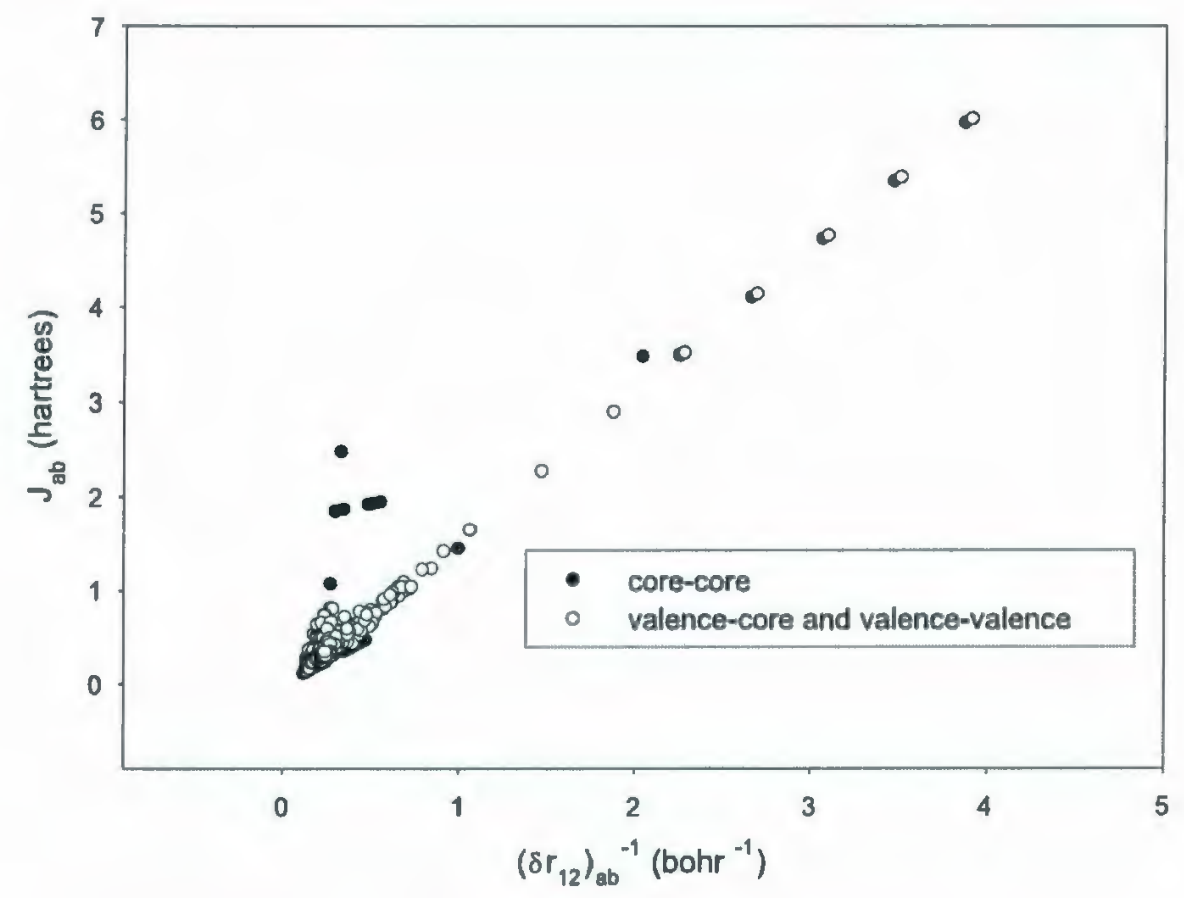

Figure 3.6. Relationship between $\left(\delta r_{12}\right)_{a b}$ and corresponding Coulomb integral, $J_{a b}$, for all systems, calculated at $\mathrm{HF} / 6-31 \mathrm{G}(\mathrm{d})$.

relationship was determined to be $J_{a b}=\frac{\alpha}{\left(\delta r_{12}\right)_{a b}}$, with $\alpha=1.56 \pm 0.01$ bohr hartrees, and $R^{2}=0.9080$. The vast majority of deviations observed are positive deviations in which the $\left(\delta r_{12}\right)_{a b}$ value is large in comparison to the corresponding $J_{a b}$ value. These deviations involve core electrons which are delocalized over two or more centres, such 
as the core carbon electrons of propane. In these cases, it appears that the delocalization causes the core electrons to experience more Coulombic repulsion from each other while maintaining a relatively large distance between them, as compared to the valence electrons. If the delocalized core-core electron pairs are removed from the plot, the value of $\alpha$ becomes $1.548 \pm 0.004$ bohr hartrees, with an $R^{2}$ value of 0.9882 . The delocalized core electrons account for the majority of the large deviations, while some smaller deviations are seen with the valence-core electron pairs. However, localized core and valence electron pairs of molecules, and all electron pairs of atoms and hydrides, follow the relationship very closely.

\section{Conclusions}

The relationship between the Coulomb integral, $J_{a b}$, and our recently formulated distance between two electrons, $\left(\delta r_{12}\right)_{a b}$, is followed extremely well by all electron pairs except core electrons delocalized over several nuclei. The relationship is also found to hold under a similarity transformation of the molecular orbitals, such as localization. The fact that a two-electron property, such as the Coulomb integral, can be closely approximated by a function of one-electron properties is intriguing and encourages the investigation of one-electron operators for other purposes, such as accounting for electron correlation. Also, in the case of the core electrons, it may be possible to incorporate a delocalization term to account for the deviations which are observed. If so, the relationship may prove to be more universal and useful. 


\section{References}

(1) E. A. Hylleraas. Z. Physik A, 54:347-366, 1929.

(2) A. A. Frost, J. Braunstein, and W. Schwemer. J. Am. Chem. Soc., 70:3292-3295, 1948.

(3) W. Klopper and W. Kutzelnigg. J. Phys. Chem., 94:5625-5630, 1990.

(4) R. Daudel, P. G. Mezey, J. D. Goddard, and I. G. Csizmadia. Can. J. Chem., 53:3739-3746, 1975.

(5) R. Daudel, J. D. Goddard, I. G. Csizmadia, E. Kapuy, and C. Kozmutza. Chem. Phys. Lett., 44:197-203, 1976.

(6) R. Daudel, J. D. Goddard, and I. G. Csizmadia. Int. J. Quant. Chem., 11:137147, 1977.

(7) M. H. Ang, K. Yates, R. Daudel, and I. G. Csizmadia. Int. J. Quant. Chem., $20: 793-806,1980$.

(8) M. R. Peterson, R. A. Poirier, R. Daudel, and I. G. Csizmadia. Int. J. Quant. Chem., 19:25-31, 1981.

(9) M. A. Robb, W. J. Haines, and I. G. Csizmadia. J. Am. Chem. Soc., 95:42-48, 1973.

(10) R. A. Poirier and J. W. Hollett. MUNgauss (Fortran 90 version). Chemistry Department, Memorial University of Newfoundland, St. John's, NL, A1B 3X7. With contributions from S. D. Bungay, A. El-Sherbiny, T. Gosse, D. Keefe, A. 
Kelly, C. C. Pye, D. Reid, K. Saputantri, M. Shaw, M.S. Staveley, Y. Wang and J. Xidos.

(11) R. Poirier, R. Kari, and I.G. Csizmadia. Handbook of Gaussian Basis Sets. Elsevier Science Publishers B.V., 1985.

(12) S.F. Boys. Quantum Theory of Atoms, Molecules and the Solid State. Academic Press, 1966. 
The following Chapter, Chapter 4, is published as an article in the Journal of Molecular Modeling.

J. W. Hollett and R. A. Poirier, J. Mol. Model., 15:739-745, 2009.

J. W. Hollett is the principal author and performed all of the research under the supervision of R. A. Poirier. 


\section{Chapter 4}

\section{Properties and applications of the average interparticle distance}

\section{Introduction}

It is generally useful to have simple physical interpretations of the theoretical electronic structure of a molecule. Specifically, the classification of molecular orbitals (MOs) as bonds, cores, and lone pairs, provides a picture of the electronic structure which is easily understood. Such a picture is obtained through localization of molecular orbitals. ${ }^{1,2}$ The molecular orbitals obtained through solving the Hartree-Fock equations are known as canonical molecular orbitals (CMOs) and, while they collectively exhibit the symmetry of the molecule (belong to irreducible representations), they are delocalized over several atoms. However, molecular orbitals may be localized by a unitary transformation using methods such as Boys ${ }^{1}$ or Ruedenberg-Edmiston ${ }^{2}$ localization. The localized molecular orbitals (LMOs) may then be used to investigate properties of a molecule which are not as conspicuous in CMOs.

Following the introduction and development of molecular orbital localization meth- 
ods, interesting properties of these orbitals were investigated and used to describe chemical phenomena. In 1973, Robb, Haines, and Csizmadia used LMOs to define the "size" of an electron pair, ${ }^{3}\left\langle r^{2}\right\rangle_{R_{a}}$.

$$
\left\langle r^{2}\right\rangle_{R_{a}}=\left|\left\langle\psi_{a}\left|r^{2}\right| \psi_{a}\right\rangle_{0}-R_{a}^{2}\right|
$$

where $r^{2}$ is the second moment operator, $\psi_{a}$ is a doubly occupied LMO, and $R_{a}$ is the centroid of charge of the LMO,

$$
\left|R_{a}\right|=\sqrt{\left\langle\psi_{a}|x| \psi_{a}\right\rangle_{0}^{2}+\left\langle\psi_{a}|y| \psi_{a}\right\rangle_{0}^{2}+\left\langle\psi_{a}|z| \psi_{a}\right\rangle_{0}^{2}}
$$

calculated at some point $R_{0}=\left(x_{0}, y_{0}, z_{0}\right)$. Csizmadia also provided a definition of the shape of an LMO. ${ }^{4}$ The second moment tensor of an LMO, $\Omega$, with respect to its own centroid of charge, is defined as

$$
\Omega=\left(\begin{array}{ccc}
{\left[\left\langle x^{2}\right\rangle_{0}-\langle x\rangle_{0}^{2}\right]} & {\left[\langle x y\rangle_{0}-\langle x\rangle_{0}\langle y\rangle_{0}\right]} & {\left[\langle x z\rangle_{0}-\langle x\rangle_{0}\langle z\rangle_{0}\right]} \\
& {\left[\left\langle y^{2}\right\rangle_{0}-\langle y\rangle_{0}^{2}\right]} & {\left[\langle y z\rangle_{0}-\langle y\rangle_{0}\langle z\rangle_{0}\right]} \\
& {\left[\left\langle z^{2}\right\rangle_{0}-\langle z\rangle_{0}^{2}\right]}
\end{array}\right) .
$$

Diagonalizing the tensor,

$$
U^{+} \Omega U=\left(\begin{array}{ccc}
\left\langle x^{2}\right\rangle & 0 & 0 \\
& \left\langle y^{2}\right\rangle & 0 \\
& & \left\langle z^{2}\right\rangle
\end{array}\right)
$$

results in three eigenvalues, $\left\langle x^{2}\right\rangle,\left\langle y^{2}\right\rangle$, and $\left\langle z^{2}\right\rangle$ which can be associated with the axes of an ellipsoid that describes the shape of the LMO. Although the shape of an LMO may have chemical implications, it is the size of an LMO which has been related to various other properties of molecules. 
It has been found that the size of a localized molecular orbital can be related to different molecular energy components. In 1975, the size of an atomic orbital, $\left\langle r^{2}\right\rangle_{i}$, was related to the orbital energy, $\epsilon_{i}$, by an equation of the form

$$
\left(\frac{1}{\epsilon_{i}}\right)=m\left\langle r^{2}\right\rangle_{i}+b
$$

where $m$ and $b$ are fitting parameters. ${ }^{5}$ The same equation was later extended to molecules, relating LMO energies to their size. ${ }^{6}$ Correlation energy was also found to be related to the size of an atom or molecule. A relationship between correlation energy, $E_{\text {corr }}$, and the total size of an atom, $\left\langle r^{2}\right\rangle$, is given by

$$
\left(\left|E_{\text {corr }}\right|\left\langle r^{2}\right\rangle\right)^{\gamma}=k(Z-\sigma),
$$

where $Z$ is the nuclear charge and $\gamma, k$, and $\sigma$ are constants optimized for each isoelectronic series of ions. ${ }^{7}$ In contrast, the total correlation energy of a molecule is related to LMO size, $\left\langle r^{2}\right\rangle_{i}$, by the equation(in a.u.)

$$
E_{\text {corr }}=-0.06593 \sum_{i=1}^{M}\left\langle r^{2}\right\rangle_{i}^{-0.1958},
$$

where $M$ is the total number of MOs. ${ }^{8}$ The relationships between LMO size and orbital energy and correlation energy may not appear obvious; however, a relationship between LMO size and steric effects should be expected.

When Robb et al. defined the size of an electron pair, ${ }^{3}$ they also investigated the energetics of rotation about the $\mathrm{CO}$ bond in $\mathrm{FCH}_{2} \mathrm{OH}$ as related to the size of the LMOs. Later, the size of an LMO was used to investigate stereochemistry in Diels-Alder reactions. Poirier et al. were able to predict facial selectivity in DielsAlder reactions of 5-substituted 1,3-cyclopentadienes using a steric factor, $S_{\mathrm{CX}} / R_{\mathrm{CX}},{ }^{9}$ 
where $X$ is the substituent, $S_{\mathrm{CX}}$ is the size of the C5-X bond, and $R_{\mathrm{C} X}$ is the distance between the centroid of the bond to C5.

The size of an $\mathrm{LMO}$, which is calculated using first and second moment operators, can model properties which require much more extensive calculation, such as orbital energy and correlation energy. Also, this easily calculated property enables the theoretical prediction of complex stereochemistry which would be much more difficult by any other method, if not impossible. Such an idea can be extended to molecules and functional groups. Using a definition similar to the size and shape of an LMO, the size and shape of a molecule or functional group can be defined, which also provides a means of calculating molecular volume. ${ }^{10}$ On the other hand, the definiton of the size of an LMO can also be generalized to include MO pairs, which may be regarded as an interelectronic distance. ${ }^{11}$ Furthermore, the interelectronic distance may be modified to represent electron-nuclear distance. In either case, the first and second moment operators prove quite useful for modeling molecular properties.

\section{Theoretical definition of the shape and size of a molecule or functional group}

Similar to the second moment tensor of an LMO (Equation 4.3), the second moment tensor of a molecule, $\mathcal{S}$, calculated at some point $r_{0}=\left(x_{0}, y_{0}, z_{0}\right)$, is given as

$$
\mathcal{S}=\left(\begin{array}{ccc}
\left\langle x^{2}\right\rangle_{r_{0}} & \langle x y\rangle_{r_{0}} & \langle x z\rangle_{r_{0}} \\
& \left\langle y^{2}\right\rangle_{r_{0}} & \langle y z\rangle_{r_{0}} \\
& & \left\langle z^{2}\right\rangle_{r_{0}}
\end{array}\right)
$$


where each element of the tensor is an expectation value of the electronic wave function. It is seen that the tensor is dependent on the selected origin, $r_{0}$, and consequently the orientation of the molecule. The origin-dependence is removed by defining an origin invariant second moment tensor, $\widetilde{\mathcal{S}}^{10}$

$$
\widetilde{\mathcal{S}}=\left(\begin{array}{ccc}
\left\langle\widetilde{x^{2}}\right\rangle & \langle\widetilde{x y}\rangle & \langle\widetilde{x z}\rangle \\
& \left\langle\widetilde{y^{2}}\right\rangle & \langle\widetilde{y z}\rangle \\
& & \left\langle\widetilde{z^{2}}\right\rangle
\end{array}\right),
$$

where

$$
\langle\tilde{i j}\rangle=\langle i j\rangle_{r_{a}}-\frac{\langle i\rangle_{r_{a}}\langle j\rangle_{r_{a}}}{N} \quad i=x, y \text {, or } z \text { and } j=x, y \text { or } z,
$$

$N$ is the number of electrons and $r_{a}$ is an arbitrary origin. The form of $\langle\tilde{i j}\rangle$ is similar to that seen in a connected moments expansion (CMX) of the energy or some other property of a wave function. ${ }^{12}$ Also, like the second moment tensor of an LMO (Equation 4.4), $\widetilde{\mathcal{S}}$ can be diagonalized,

$$
\widetilde{\mathcal{S}}^{\prime}=\left(\begin{array}{ccc}
\left.Q^{+} \widetilde{\mathcal{S}} Q=\widetilde{x^{\prime 2}}\right\rangle & 0 & 0 \\
& \left.\widetilde{y^{\prime 2}}\right\rangle & 0 \\
& & \left.\widetilde{z^{\prime 2}}\right\rangle
\end{array}\right) .
$$

The eigenvalues, $\left\langle\widetilde{x^{2}}\right\rangle,\left\langle\widetilde{y^{12}}\right\rangle$ and $\left\langle\widetilde{z^{12}}\right\rangle$, correspond to the principal axes of the electronic second moment of the molecule, $Q$, and can be associated with the major and minor axes of an ellipsoid that describes the shape of the molecule. A quantitative measure of size may be calculated from the eigenvalues, such as the geometric average of the axes,

$$
\widetilde{R}=\left(\left\langle\widetilde{x^{\prime 2}}\right\rangle\left\langle\widetilde{y^{/ 2}}\right\rangle\left\langle\widetilde{z^{2}}\right\rangle\right)^{1 / 6}
$$


and the molecular volume,

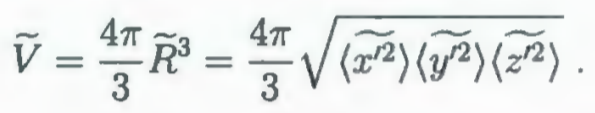

The size or volume of a functional group, or substituent, $X$ may be defined as the size or volume of the molecule $\mathrm{H} X$, where the volume of $\mathrm{H}$ is taken to be constant.

An illustration of molecular sizes and shapes, calculated at $\mathrm{HF} / 6-31 \mathrm{G}(\mathrm{d})$, is given in Figure 4.1. As expected, the $\mathrm{H}_{2}$ molecule is larger in the z-direction, $2.151 \mathrm{bohr}^{2}$, and symmetric in the xy-plane, $1.507 \mathrm{bohr}^{2}$. It is seen that water is largest in the y-direction, 7.113 bohr $^{2}$, which corresponds to the direction of a line passing through the two hydrogen atoms. For methanol and formic acid, it is seen that both molecules are significantly larger along the y-axis, $50.256 \mathrm{bohr}^{2}$ and $93.234 \mathrm{bohr}^{2}$ respectively, which is the general direction of their CO bonds. As expected the volume of the molecules increase in the order $\mathrm{H}_{2}\left(\widetilde{V}=0.83 \mathrm{~cm}^{3} \mathrm{~mol}^{-1}\right), \mathrm{H}_{2} \mathrm{O}\left(\widetilde{V}=5.78 \mathrm{~cm}^{3} \mathrm{~mol}^{-1}\right)$, $\mathrm{CH}_{3} \mathrm{OH}\left(\widetilde{V}=42.94 \mathrm{~cm}^{3} \mathrm{~mol}^{-1}\right)$, to $\mathrm{CHOOH}\left(\widetilde{V}=64.43 \mathrm{~cm}^{3} \mathrm{~mol}^{-1}\right)$.

This origin invariant formulation for the size of a molecule is also found to correlate quite well with another theoretical measure of molecular volume. A significantly more complex method of calculating molecular volume is through use of isodensity contours. During the development of the theory of atoms and molecules, Bader et al. investigated the volume of molecules using isodensity contours. ${ }^{13}$ Upon comparing the origin invariant volume, $\widetilde{V}$, to the Bader volume, $V_{\rho}$, it is found that $\widetilde{V}$ is smaller than $V_{\rho}$ for small molecules and significantly larger than $V_{\rho}$ for larger molecules. This disproportional growth of $\tilde{V}$ relative to $V_{\rho}$ is due to the behaviour of the second moment operator, $r^{2}{ }^{10}$ A linear relationship was found to exist between $V_{\rho}$ and the geometric average of the principle axes of the origin invariant electronic second 

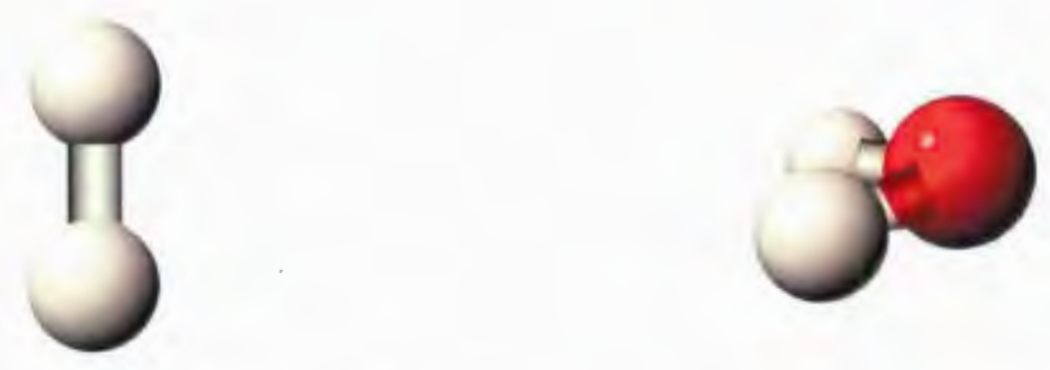

$$
\begin{aligned}
& \widetilde{\left\langle x^{2}\right\rangle}=1.507 \mathrm{bohr}^{2} \\
& \frac{\left\langle y^{2}\right\rangle}{\widetilde{\left\langle z^{2}\right\rangle}}=1.507 \mathrm{bohr}^{2} \\
& \widetilde{R}=1.151 \mathrm{bohr}^{2} \\
& \widetilde{V}=0.83 \mathrm{~cm}^{3} \mathrm{~mol}^{-1}
\end{aligned}
$$

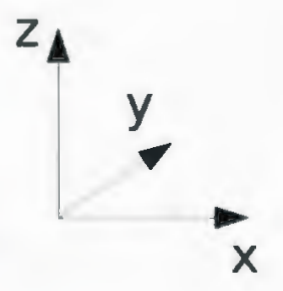

$$
\begin{aligned}
\widetilde{\left\langle x^{2}\right\rangle} & =6.265 \mathrm{bohr}^{2} \\
\frac{\left\langle y^{2}\right\rangle}{\left\langle z^{2}\right\rangle} & =7.113 \mathrm{bohr}^{2} \\
\widetilde{R} & =2.357 \mathrm{bohr}^{2} \\
\widetilde{V} & =5.491 \mathrm{bohr} \\
& =5.78 \mathrm{~cm}^{3} \mathrm{~mol}^{-1}
\end{aligned}
$$
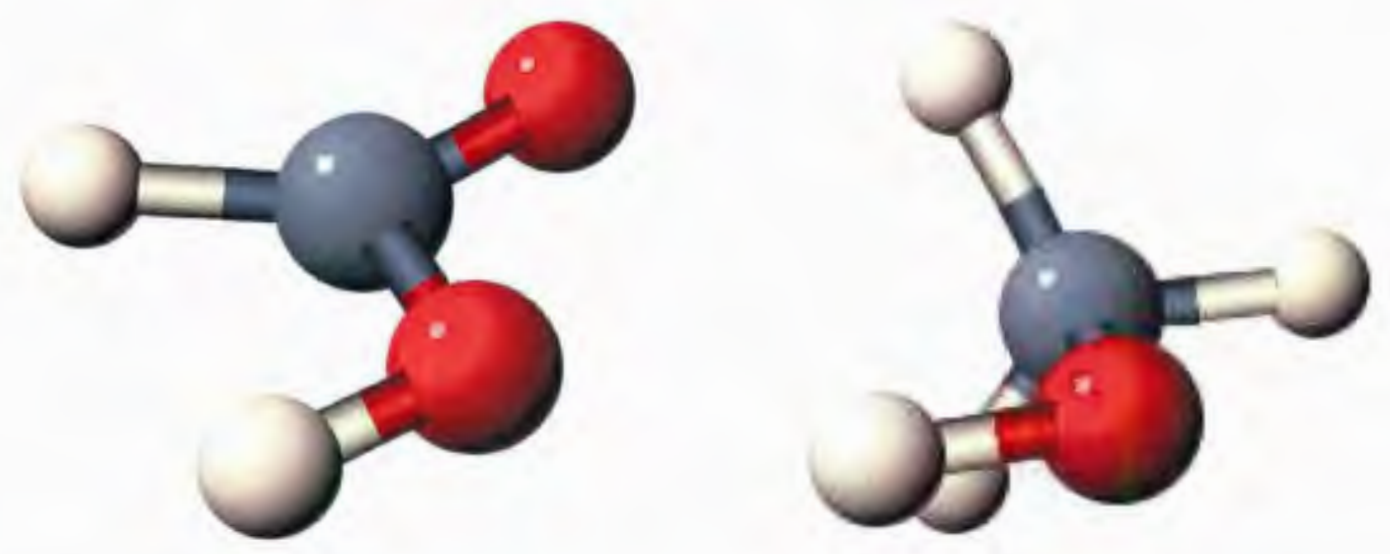

$$
\begin{aligned}
\widetilde{\left\langle x^{2}\right\rangle} & =25.834 \mathrm{bohr}^{2} \\
\frac{\left\langle y^{2}\right\rangle}{\left\langle z^{2}\right\rangle} & =93.234 \mathrm{bohr}^{2} \\
\widetilde{R} & =12.333 \mathrm{bohr}^{2} \\
\widetilde{V} & =64.565 \mathrm{bohr}^{3}
\end{aligned}
$$

$$
\begin{aligned}
\widetilde{\left\langle x^{2}\right\rangle} & =16.714 \mathrm{bohr}^{2} \\
\frac{\left\langle y^{2}\right\rangle}{\widetilde{\left\langle z^{2}\right\rangle}}= & =15.256 \mathrm{bohr}^{2} \\
\widetilde{R} & =4.8612 \mathrm{bohr}^{2} \\
\widetilde{V} & =42.94 \mathrm{~cm}^{3} \mathrm{~mol}^{-1}
\end{aligned}
$$

Figure 4.1. The shape and size of some small molecules $\left(\mathrm{H}_{2}, \mathrm{H}_{2} \mathrm{O}, \mathrm{CH}_{3} \mathrm{OH}\right.$, and $\left.\mathrm{CHOOH}\right)$ calculated at $\mathrm{HF} / 6-31 \mathrm{G}(\mathrm{d})$. Molecules are slightly rotated off the indicated principal axes to show all atoms. 
moment, $\widetilde{R}$.

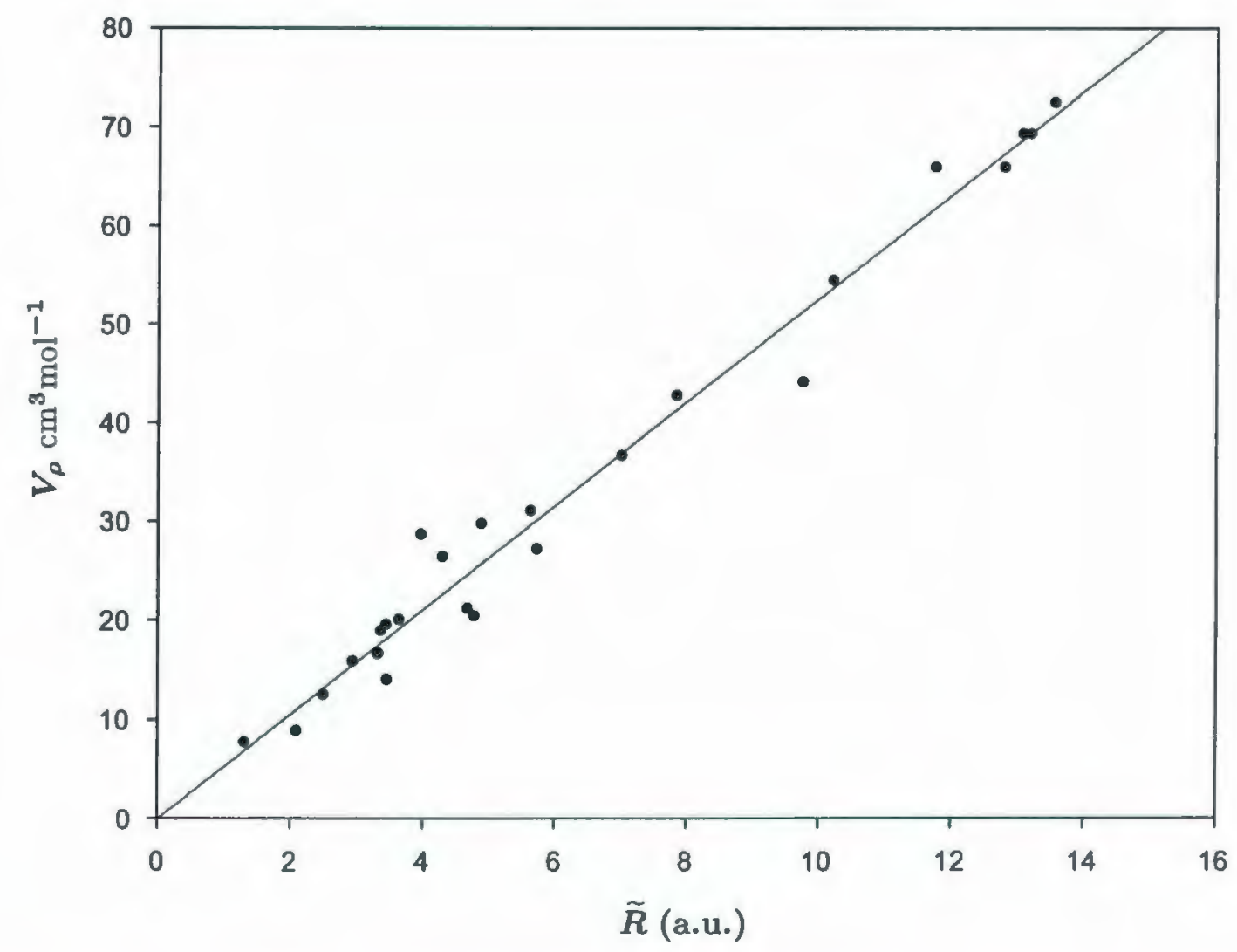

Figure 4.2. Comparison of electronic second moment average radius, $\widetilde{R}(\mathrm{HF} / 6-31 \mathrm{G}(\mathrm{d}))$, to Bader isodensity contour molecular volume, ${ }^{13} V_{\rho}(\rho=0.002$ a.u. $)$. From reference 10.

The linear relationship,

$$
V_{\rho}=\alpha \widetilde{R}
$$

for a set of 26 molecules and $V_{\rho}$ values for an isodensity contour of 0.002 a.u. is shown in Figure 4.2. The value of $\alpha$ is $5.45 \pm 0.15 \mathrm{~cm}^{3} \mathrm{~mol}^{-1} \mathrm{bohr}^{-1}$ and $R^{2}=0.982 .{ }^{10}$ The measure of molecular size using the electronic second moment, $\widetilde{R}$, is comparable to 
the molecular volume from isodensity contours, $V_{\rho}$, yet it is significantly easier to calculate the second moment of a molecule than trace an isodensity contour and calculate its volume.

The theoretical measure of the size of a functional group, $\widetilde{R}$, also correlates quite well with experimental measures of steric hindrance. It was found that $\widetilde{R}$ correctly predicts the trend of $P$-values of the halogens and hydrogen, ${ }^{10}$ where $P$-values are steric factors measured from the rotational barriers of substituted ethanes. ${ }^{14}$ Another measure of steric hindrance which is determined from a conformational process is $n$ value. The free energy of topomerization of a phane system with an intra-annular substituent $X$, is used to determine the $n$-value for $X .^{15}$ When the available $n$-values for a set of substituents is compared to their theoretical size, $\widetilde{R}$, the same trend is predicted, with the exception of $\mathrm{NO}_{2} \cdot{ }^{10}$ It is expected that the deviation from the observed trend is due to effects other than sterics, such as electronic interaction of the oxygens of $\mathrm{NO}_{2}$ with the aliphatic chain of the phane system.

The theoretical measures of size, $\widetilde{R}$ and $\widetilde{V}$, are easily calculated from the first and second moments of the electronic wave function. Another theoretical measure of size, which is substantially more complex, $V_{\rho}$, correlates quite well with $\widetilde{R}$. The geometric average of the principal axes, $\widetilde{R}$, is also capable of predicting experimentally measured steric effects. These models of the simple concepts "size" and "shape" are obtained through use of the operators $r$ and $r^{2}$, which, at the molecular orbital level, can be used to model interparticle distances. 


\section{The average interparticle distance}

\subsection{The average interelectronic distance}

The average distance between an electron in $\mathrm{MO} a$ and an electron in MO $b$, is given by ${ }^{11}$

$$
\left(\delta r_{12}\right)_{a b}=\sqrt{\begin{array}{l}
\left\langle x^{2}\right\rangle_{a}+\left\langle x^{2}\right\rangle_{b}-2\langle x\rangle_{a}\langle x\rangle_{b} \\
+\left\langle y^{2}\right\rangle_{a}+\left\langle y^{2}\right\rangle_{b}-2\langle y\rangle_{a}\langle y\rangle_{b} \\
+\left\langle z^{2}\right\rangle_{a}+\left\langle z^{2}\right\rangle_{b}-2\langle z\rangle_{a}\langle z\rangle_{b}
\end{array}}=\sqrt{\left\langle a b\left|r_{12}^{2}\right| a b\right\rangle},
$$

where

$$
\langle\mu\rangle_{a}=\langle a|\mu| a\rangle, \quad \mu=x, y, \text { or } z .
$$

Although this measure is not strictly the expectation value of $r_{12}$ (hence the italicized average), it does give a measure of the charge distribution of a single $\mathrm{MO}$, or the interaction between separate MOs. It is important to note that for LMOs, if the electrons occupy the same $\mathrm{MO}, a=b,\left(\delta r_{12}\right)_{a a}$ reduces to the definition of the size of an electron pair (Equation 4.1), multiplied by $\sqrt{2}$.

$$
\left(\delta r_{12}\right\rangle_{a a}=\sqrt{\begin{array}{c}
2\left\langle x^{2}\right\rangle_{a}-2\langle x\rangle_{a}^{2} \\
+2\left\langle y^{2}\right\rangle_{a}-2\langle y\rangle_{a}^{2} \\
+2\left\langle z^{2}\right\rangle_{a}-2\langle z\rangle_{a}^{2}
\end{array}}=\sqrt{2}\left\|\delta r_{a}\right\|
$$

This value is also the root mean square deviation in the position of an electron in MO $a$, multiplied by $\sqrt{2}$. Unlike the original size of an electron pair (Equation 4.1), $\left(\delta r_{12}\right)_{a b}$ is generalized to pairs of MOs and is applicable to CMOs, as well as LMOs. To illustrate the behaviour of such a quantity, the value of $\left(\delta r_{12}\right)_{a a}$ is calculated for the singlet and triplet states of a series of two-electron systems. 


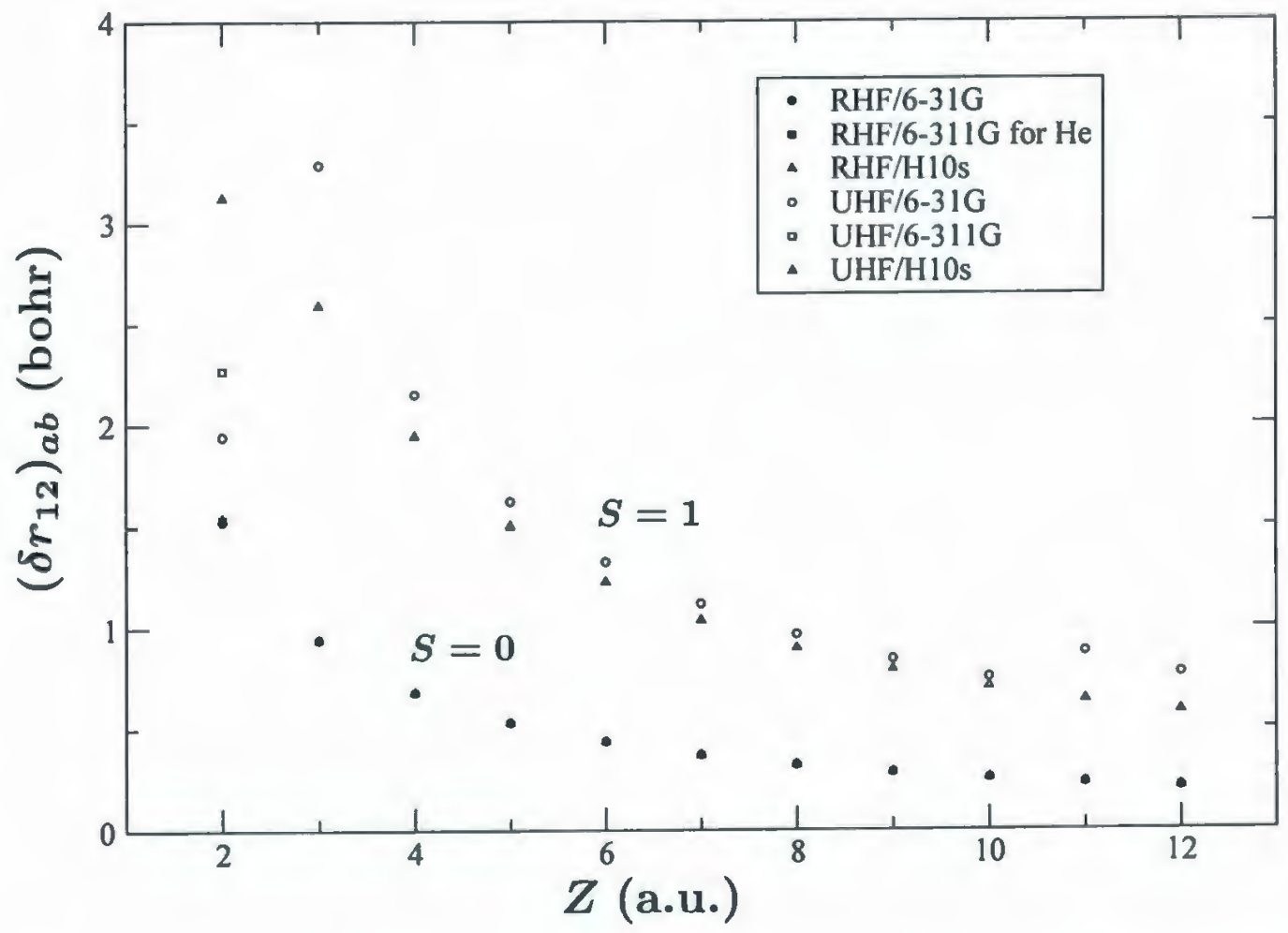

Figure 4.3. $\left(\delta r_{12}\right)_{a b}$ of two-electron singlet and triplet systems with varying nuclear charge (He to $\mathrm{Mg}^{10+}$ ). Note that some points overlap. 
It is seen that for both the singlet and triplet states the distance between the electrons decreases smoothly with increasing nuclear charge, with the exception of the UHF/6-31G triplet systems(Figure 4.3). The discrepancy seen is due to the inconsistency of the 6-31G basis set, which for He has only two s-type functions compared to three s-type functions for the first-row elements. When an extra basis function is added to $\mathrm{He}$, by using the $6-311 \mathrm{G}$ basis set, the $\left(\delta r_{12}\right)_{a b}$ value is increased which agrees more with the trend. A similar effect is seen upon going from $\mathrm{Ne}^{8+}$ to $\mathrm{Na}^{9+}$, which is due to the difference in the 6-31G basis set for second and first-row

elements. If a Huzinaga basis set with ten s-type functions, ${ }^{16} \mathrm{H} 10 \mathrm{~s}$, is used for all atoms, a much better trend is observed. In the case of the singlet states, it is seen that basis set has little effect on the value of $\left(\delta r_{12}\right)_{a a}$ of the two-electron systems(Figure 4.3). It should also be noted that as expected, $\left(\delta r_{12}\right)_{a b}^{\text {singlet }}<\left(\delta r_{12}\right)_{a b}^{\text {triplet }}$.

\section{2 $\left(\delta r_{12}\right)_{a b}$ and the corresponding Coulomb and exchange integrals}

Similar to the definition of the size of an LMO, the average distance between electrons is related to a component of the energy, namely the Coulomb energy. One might expect the repulsion between two electrons, $J_{a b}$, to be inversely proportional to the distance between them, $\left(\delta r_{12}\right)_{a b}$, which is indeed the case. The Coulomb integral, $J_{a b}$, is a two-electron integral,

$$
J_{a b}=\langle a b \mid a b\rangle
$$

which is related to $\left(\delta r_{12}\right)_{a b}$, a property calculated from one-electron expectation values, by the equation

$$
J_{a b} \approx \frac{\alpha}{\left(\delta r_{12}\right)_{a b}}
$$

The relationship between $\left(\delta r_{12}\right)_{a b}$ and $J_{a b}$ holds for CMOs and LMOs. However, 


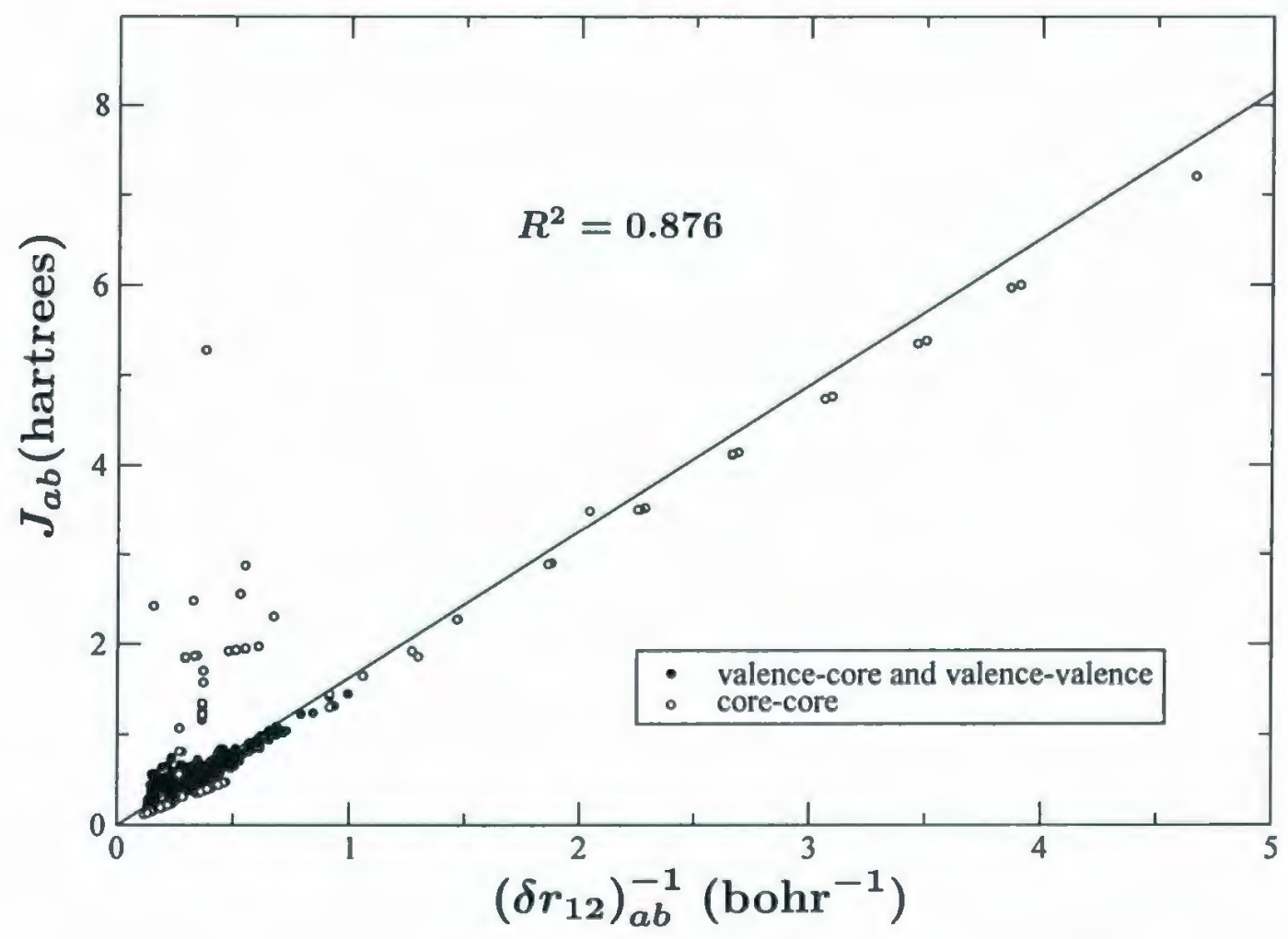

Figure 4.4. Relationship between Coulomb energy, $J_{a b}$, and the average distance between two electrons, $\left(\delta r_{12}\right)_{a b}$, for two-electron ions to molecules with up to 58 electrons (HF/6-31G(d), CMO). 


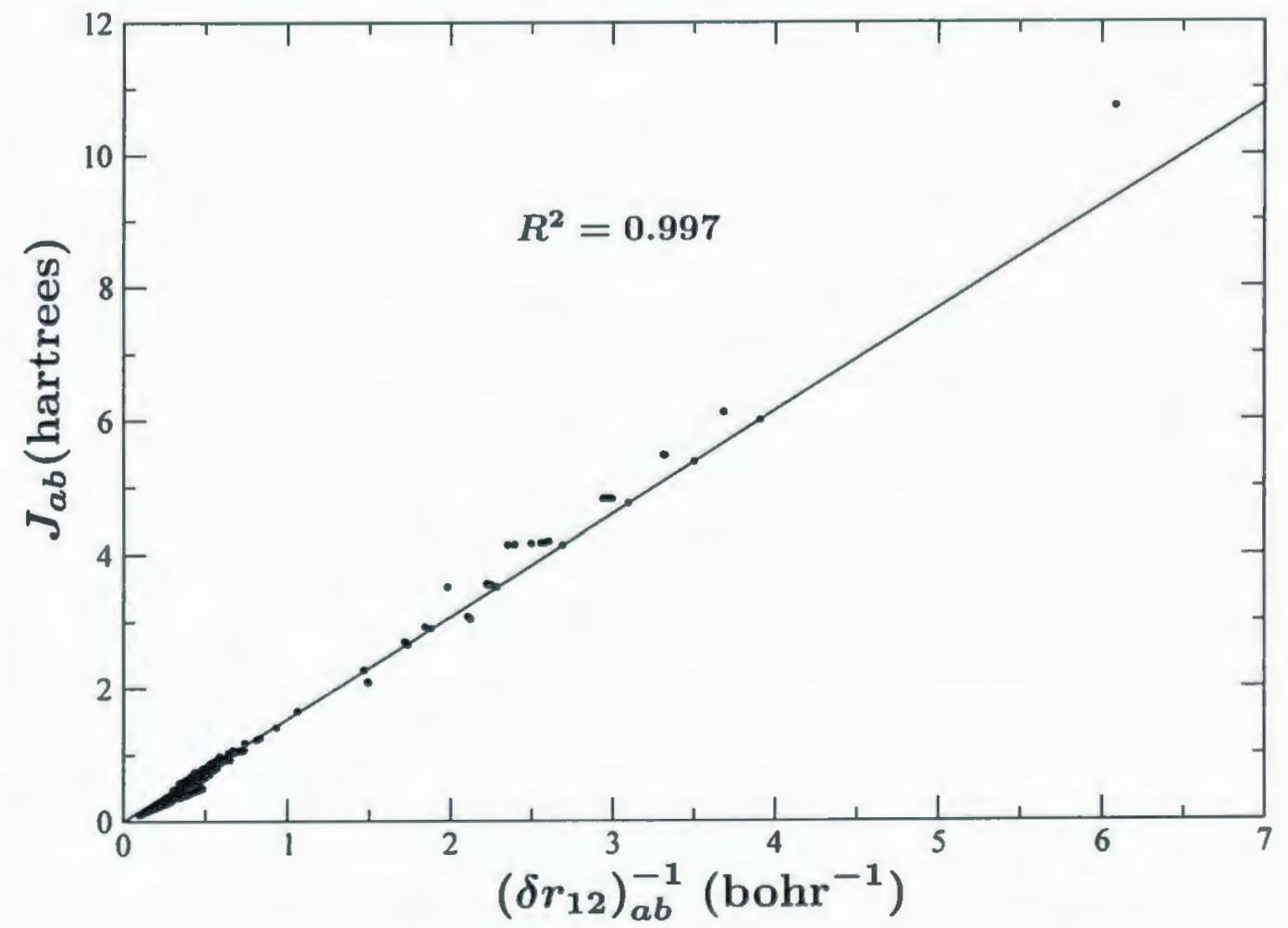

Figure 4.5. Relationship between Coulomb energy, $J_{a b}$, and the average distance between two electrons, $\left(\delta r_{12}\right)_{a b}$, for two-electron ions to molecules with up to 58 electrons (HF/6-31G(d), LMO). 
there are significant deviations in the relationship when dealing with delocalized core CMOs (Figure 4.4). For example, the $\left(\delta r_{12}\right)_{a b}$ value for the two lowest energy CMOs of ethane, which involve the cores of the carbons, is too large and predicts a $J_{a b}$ value that is far lower than the actual HF value. When the molecular orbitals are localized, there are no instances of delocalized cores and the relationship is followed much more closely. The relationship using LMOs for the various systems studied (at $\mathrm{HF} / 6-31 \mathrm{G}(\mathrm{d})$ ), which includes two-electron ions to 58 electron molecules, has an $\alpha$ (Equation 4.20) value of $1.537 \pm 0.002 \mathrm{bohr}$ hartrees, and $R^{2}=0.997$ (Figure 4.5). Among these molecules are carbon dioxide, propane, acetic acid, hydrogen cyanide, benzene, and uracil. The largest deviation seen in Figure 4.4 is actually three points due to the two lowest energy core MOs, 1 and 2 , of $\mathrm{Cl}_{2}$. The value of $\mathrm{J}_{11}, \mathrm{~J}_{21}$ and $\mathrm{J}_{22}$ are equivalent as are the corresponding $\left(\delta r_{12}\right)_{a b}$ values. The underestimation of $\mathrm{J}_{a b}$ is due to the large $\left(\delta r_{12}\right)_{a b}$ value, which is a result of the delocalization of MOs 1 and 2 onto both chlorine atoms. The internuclear distance, and the lack of MO density between the atoms, leads to an $\left(\delta r_{12}\right)_{a b}$ value that does not predict the corresponding $\mathrm{J}_{a b}$ value. An even larger deviation would be seen for the core MOs of $\mathrm{Br}_{2}$. The $\mathrm{J}_{a b}$ values involving delocalized core MOs could be more accurately modeled using a $\left(\delta r_{12}\right)_{a b}$ value which is a sum of atomic contributions. For core MOs localized on one atom, or valence MOs over multiple atoms this type of deviation does not occur.

It has also been found, that for simple systems, the exchange integral, $K_{a b}$, can also be modeled using $\left(\delta r_{12}\right)_{a b}$.

$$
K_{a b}=\langle a b \mid b a\rangle,
$$

The relationship between $K_{a b}$ and $\left(\delta r_{12}\right)_{a b}$ for three and four-electron atoms and 


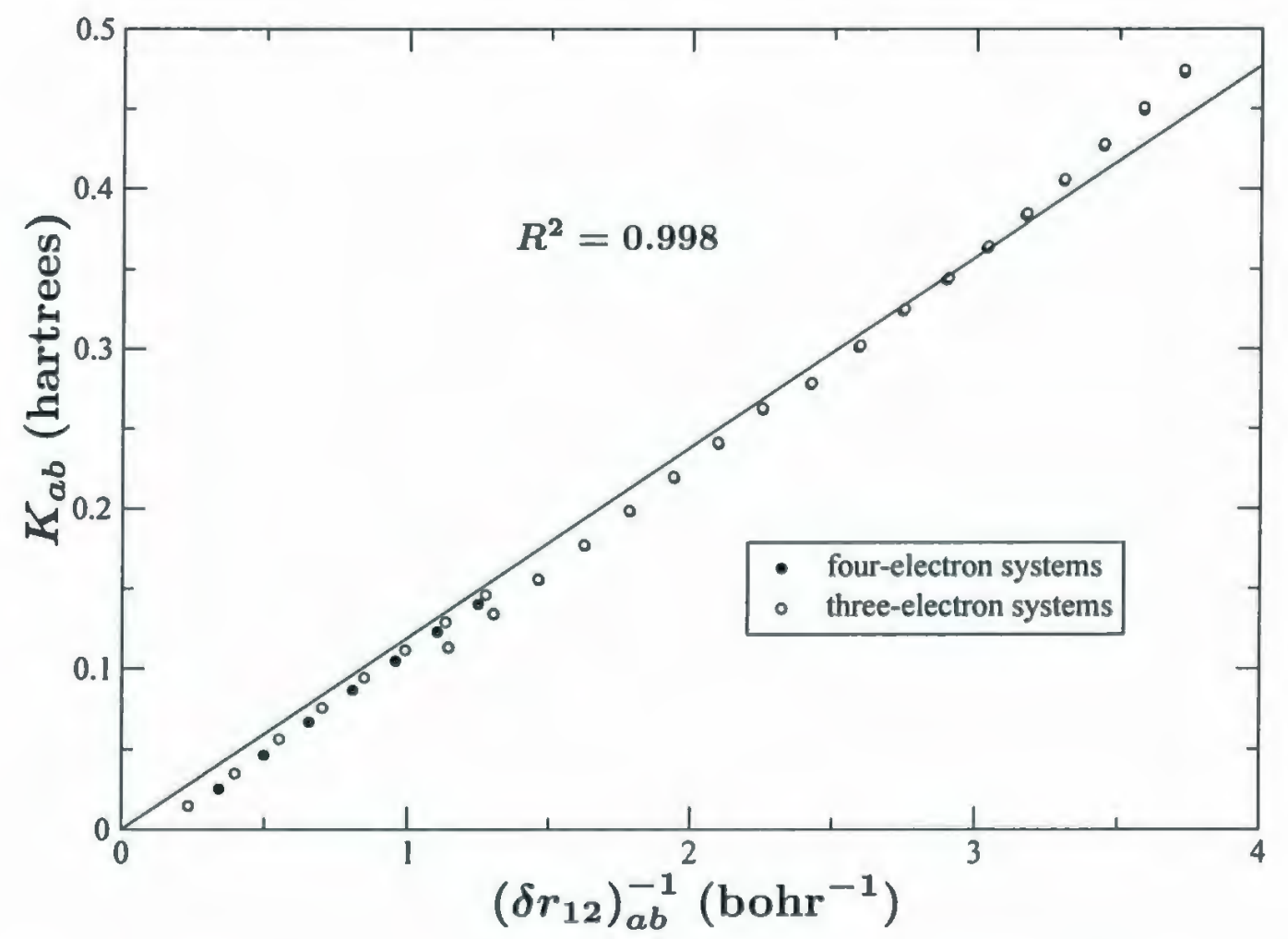

Figure 4.6. Relationship between exchange energy, $K_{a b}$, and the average distance between two electrons, $\left(\delta r_{12}\right)_{a b}$, for three and four-electron systems with varying nuclear charge (ROHF/6-31G(d)). 
ions has the same form as the relationship with $J_{a b}$.

$$
K_{a b} \approx \frac{\beta}{\left(\delta r_{12}\right)_{a b}}
$$

There is a slight overlap of the data points in the series of three and four-electron systems (Figure 4.6) which is due to the difference in the 6-31G(d) basis set for the first and second-row elements. Despite the uneven basis set, $\left(\delta r_{12}\right)_{a b}$ models $K_{a b}$ quite well in these systems, with $\beta=0.1193 \pm 0.0011$ bohr hartrees and $R^{2}=0.998$. The ability to model a two-electron property such as the Coulomb or exchange energy with a quantity which is simply calculated from one-electron expectation values is an important result. Calculation of the two-electron integrals which are required for the Coulomb and exchange energies, $J_{a b}$ and $K_{a b}$, is generally the most time consuming part of an ab initio calculation.

\subsection{The average electron-nucleus distance and the electron-nucleus potential energy}

If the expression for the distance between two electrons, $\left(\delta r_{12}\right)_{a b}$, is modified by replacing the expectation values of $\mathrm{MO} b$ with coordinates of nucleus $A$, an expression for the average distance between an electron in $\mathrm{MO} a$ and nucleus $A$ is obtained, $\delta r_{a A}$.

$$
\delta r_{a A}=\sqrt{\sum_{\mu=x, y, z}\left\langle a\left|\mu^{2}\right| a\right\rangle+\mu_{A}^{2}-2\langle a|\mu| a\rangle \mu_{A}}=\sqrt{\left\langle a\left|r_{1 A}^{2}\right| a\right\rangle}
$$

where $\mu_{A}$ are the coordinates of nucleus $A$. As expected there is a relationship between $\delta r_{a A}$ and the electron-nucleus attraction potential energy associated with an electron in $\mathrm{MO} a, V_{a a}$.

$$
V_{a a}=\left\langle a\left|\sum_{A=1}^{M} \frac{-Z_{A}}{r_{1 A}}\right| a\right\rangle
$$


Similar to $\left(\delta r_{12}\right)_{a b}$ and $J_{a b}$, the relationship holds for CMOs and LMOs, with the exception of the delocalized core CMOs.

$$
V_{a a}=\gamma \sum_{A=1}^{M} \frac{-Z_{A}}{\delta r_{a A}}
$$

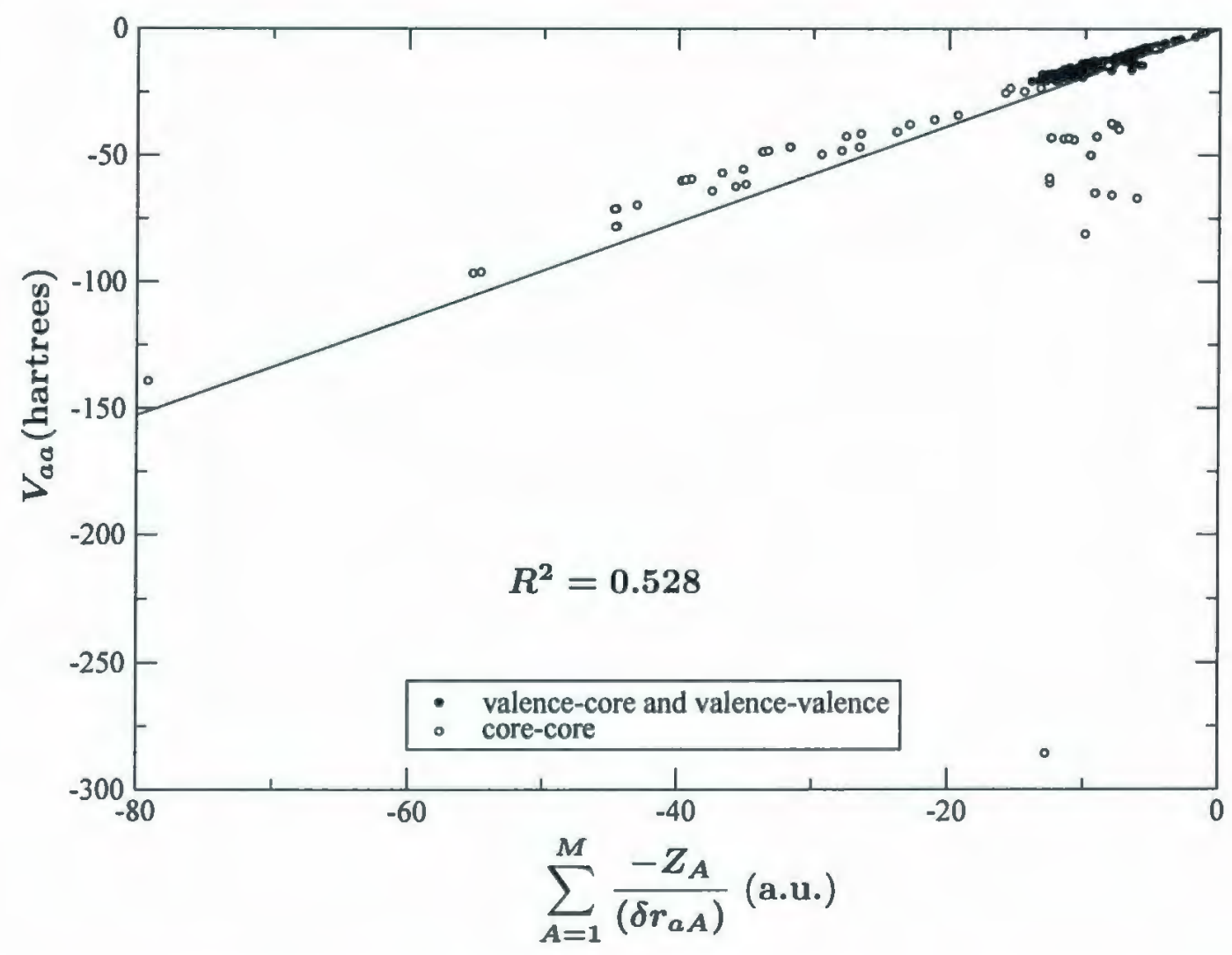

Figure 4.7. Relationship between electron-nucleus potential energy, $V_{a a}$, and the average distance between an electron and a nucleus, $\delta r_{a A}$, for two-electron ions to molecules with up to 58 electrons (HF/6-31G(d), CMO).

The largest deviation seen in Figure 4.7 is due the two lowest energy core MOs of $\mathrm{Cl}_{2}$, as seen with the relationship between $J_{a b}$ with $\left(\delta r_{12}\right)_{a b}$ (Figure 4.4). The electron-nucleus potential energy is calculated from one-electron integrals, therefore modeling $V_{a a}$ with $\delta r_{a A}$ does not have the same implications as modeling $J_{a b}$ with 


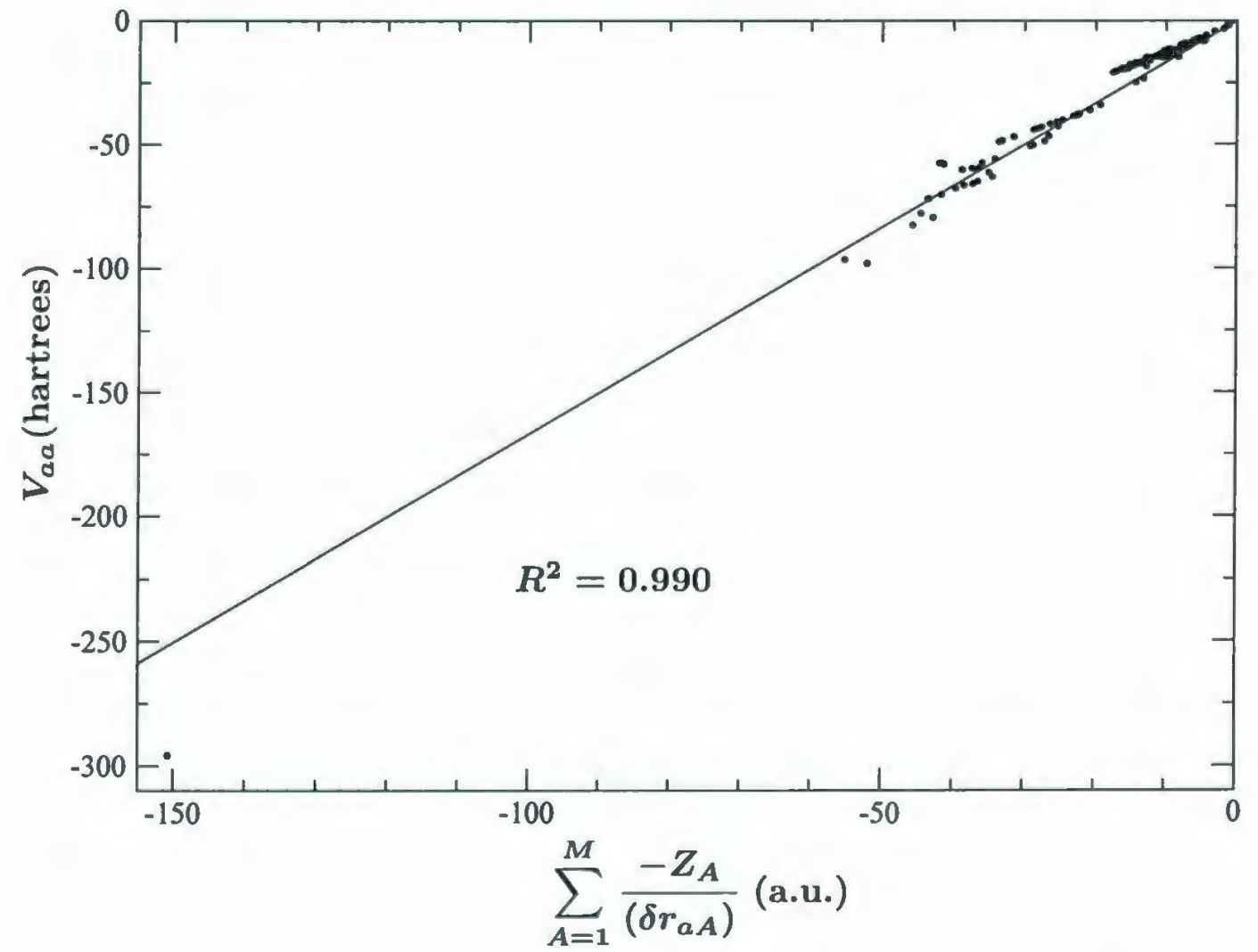

Figure 4.8. Relationship between electron-nucleus potential energy, $V_{a a}$, and the average distance between an electron and a nucleus, $\delta r_{a A}$, for two-electron ions to molecules with up to 58 electrons (HF/6-31G(d), LMO). 
$\left(\delta r_{12}\right)_{a b}$. However, the model does demonstrate how, once again, energy components may be modeled using first and second moment operators. These findings, sections 3.2 and 3.3, have important consequences. It has been shown that these interparticle distances share an approximate linear relationship with interatomic distance. This result combined with the relationships presented here form a basis for a novel approach to molecular modeling, Simulated Electronic Structure Theory (SEST). ${ }^{17}$ In SEST the energy components associated with individual electrons are expressed as functions of the nuclear coordinates. An empirical model which explicitly includes the electrons has several advantages over conventional molecular mechanics.

\section{Conclusions}

Since the theoretical definition of the size of an electron pair was introduced several relationships involving LMO size, energy and stereochemistry have been discovered. However, the idea behind the definition, the use of first and second moment expectation values as a measure of the size of electronic structure, can be further modified

for various other purposes. As seen in this study application of this idea of size to an entire molecule resulted in a theoretical definition of the size of a molecule or functional group. Such a measure, which is origin invariant, is useful for describing chemical structures as well as predicting steric effects in molecules and reactions. The size of an LMO may be generalized to pairs of MOs, as an expression for the distance between two electrons, $\left(\delta r_{12}\right)_{a b}$, which also applies to CMOs. Such a property enables the modeling of a complex two-electron property, $J_{a b}$, using simple one-electron expectation values. The model performs significantly better for LMOs than CMOs. Finally, the formalism of the distance between two electrons can be applied to an 
electron and a nucleus to give, $\delta r_{a A}$. This property is also related to an energy component, the electron-nucleus attraction energy, $V_{a a}$. Relationships such as these could prove useful in molecular modeling on a larger scale. ${ }^{17}$ Furthermore, the success in using first and second moment operators to model the Coulomb and electron-nucleus potential energy, and exchange energy, of a molecule certainly merits investigation into the modeling of other properties, as well as the investigation into higher order moments and their behaviour.

\section{Acknowledgements}

Work supported by Natural Sciences and Engineering Research Council of Canada. Computational facilities are provided by Atlantic Computational Excellence Network. 


\section{References}

(1) S.F. Boys. Quantum Theory of Atoms, Molecules and the Solid State. Academic Press, 1966.

(2) C. Edmiston and I. Ruedenberg. Rev. Mod. Phys., 35:457-465, 1963.

(3) M. A. Robb, W. J. Haines, and I. G. Csizmadia. J. Am. Chem. Soc., 95:42-48, 1973.

(4) I. G. Csizmadia. Localization and Delocalization in Quantum Chemistry, 1:349$353,1975$.

(5) R. Daudel, P. G. Mezey, J. D. Goddard, and I. G. Csizmadia. Can. J. Chem., 53:3739-3746, 1975.

(6) R. Daudel, J. D. Goddard, and I. G. Csizmadia. Int. J. Quant. Chem., 11:137$147,1977$.

(7) M. H. Ang, K. Yates, R. Daudel, and I. G. Csizmadia. Int. J. Quant. Chem., 20:793-806, 1980.

(8) M. R. Peterson, R. A. Poirier, R. Daudel, and I. G. Csizmadia. Int. J. Quant. Chem., 19:25-31, 1981.

(9) J. D. Xidos, R. A. Poirier, C. C. Pye, and J. Burnell. J. Org. Chem., 63:105-112, 1998.

(10) J. W. Hollett, A. Kelly, and R. A. Poirier. J. Phys. Chem. A, 110:13884-13888, 2006. 
(11) J. W. Hollett and R. A. Poirier. J. Theoretical Computational Chem., 6:13-22, 2007.

(12) J. Cioslowski. Phys. Rev. Lett., 58:83-85, 1987.

(13) R. F. W. Bader, M. T. Carrol, J. R. Cheeseman, and C. Chang. J. Am. Chem. Soc., 109:7968-7979, 1987.

(14) J. E. Anderson and H. Pearson. J. Chem. Soc. Chem. Comm., pages 871-872, 1971.

(15) H. Förster and F. Vögtle. Angew. Chem. Int. Ed. Engl., 16:429-441, 1977.

(16) R. Poirier, R. Kari, and I.G. Csizmadia. Handbook of Gaussian Basis Sets. Elsevier Science Publishers B.V., 1985.

(17) J. W. Hollett and R. A. Poirier. J. Chem. Theory Comput., 5:126-135, 2009. 
The following Chapter, Chapter 5, is published as an article in the Journal of Chemical Theory and Computation.

J. W. Hollett and R. A. Poirier, J. Chem. Theory Comput., 5:126-135, 2009.

J. W. Hollett is the principal author and performed all of the research under the supervision of R. A. Poirier. 


\section{Chapter 5}

\section{SEST: Simulated Electronic}

\section{Structure Theory}

Molecular mechanics methods are widely used to study problems in a variety of research fields, from nanotechnology ${ }^{1}$ to molecular biology. ${ }^{2}$ In conventional molecular mechanics, the total energy of a molecule is divided into different components; bond stretching, $E_{\mathrm{s}}$, angle bending, $E_{\mathrm{b}}$, torsional interactions, $E_{\text {tor }}$, van der Waals interactions, $E_{\mathrm{vdW}}$, and electrostatic interactions, $E_{\text {elec }}{ }^{3}$

$$
E_{\text {tot }}(\mathbf{R})=E_{\mathrm{s}}+E_{\mathrm{b}}+E_{\text {tor }}+E_{\mathrm{vdW}}+E_{\text {elec }}
$$

Some formulations may use other energy terms to describe phenomena, such as hydrogen bonding, that are not described well by the existing terms. The functional form of these energy terms and the empirical parameters that occur in the functions are known as a force field. Given the functional form of a molecular mechanics force field, the empirical parameters are defined through fitting of experimental or $a b$ initio data. 
The major advantage of molecular mechanics methods over $a b$ initio, density functional, or semi-empirical methods is their computational efficiency. Molecular mechanics allows for the study of large systems and dynamics where it is not feasible or strictly impossible to use other approaches. The flexibility of force fields which may be tuned to various classes of compounds allows for quite accurate calculations. However, on the other hand, the specificity of the force fields makes studies on unknown compounds inaccurate and unpredictable. This lack of transferability of specific atom-atom interactions is due to contamination by the molecular environment, which is, indirectly, a result of neglecting the electronic structure. The absence of electrons and orbitals also poses problems for chemical calculations. It is not possible to study reaction mechanisms with molecular mechanics, or many other properties related to the electronic structure, such as; magnetic properties, excitations, electron transport and electrical conductivity. A partial solution to such a problem is through a combined quantum mechanics and molecular mechanics (QM/MM) approach. ${ }^{4}$

A hybrid QM/MM approach is common in the study of reactions involving large systems, such as enzyme catalysis or organic reactions in solution. The general scheme behind a $\mathrm{QM} / \mathrm{MM}$ calculation is to treat the region of interest, or importance, with a quantum mechanical calculation and the rest of the system is treated with molecular mechanics. In some applications, the solvent may be treated with molecular mechanics while the reacting molecules are treated quantum mechanically, or for large molecules, like enzymes, the active site may be QM while the rest of the molecule is MM. The Hamiltonians and energy of these hybrid systems are divided into QM, $\mathrm{MM}$, and $\mathrm{QM} / \mathrm{MM}$ parts. How the two different regions interact, QM/MM, is defined in several ways. ${ }^{5}$ 
Since the initial appearance of molecular mechanics almost a century ago, ${ }^{6}$ most of the development of the theory has been focused on parametrization (i.e. developing force fields for different classes of compounds) while the general formulation of molec-

ular mechanics has remained relatively the same. This has led to accurate modelling of a wide variety of large systems in many research fields. However, the problems associated with the absence of electronic structure are still present. While methods such as $\mathrm{QM} / \mathrm{MM}$ provide a partial solution to such a problem, there may be other approaches. This study explores such an idea. Rather than the molecular mechanics approach of molecular modelling, modelling of electronic structure is performed. The theory presented provides a different approach to modelling potential energy surfaces, where the energy components associated with electron pairs and individual electrons are functions of the nuclear coordinates.

\section{Theory}

\subsection{Simulated Electronic Structure Theory (SEST)}

A theory which includes electrons explicitly will have an energy expression that differs significantly from conventional molecular mechanics (Equation 5.1) and more closely resemble the electronic Hamiltonian (Equation 5.2, in atomic units).

$$
\hat{H}_{\mathrm{e}}=-\sum_{i=1}^{N} \frac{1}{2} \nabla_{i}^{2}-\sum_{i=1}^{N} \sum_{A=1}^{M} \frac{Z_{A}}{r_{i A}}+\sum_{i=1}^{N} \sum_{j>i}^{N} \frac{1}{r_{i j}}
$$

where $N$ is the number of electrons and $M$ is the number of nuclei. The SEST energy expression can be expressed as a sum of atomic contributions, $V_{\text {atomic }}$, and

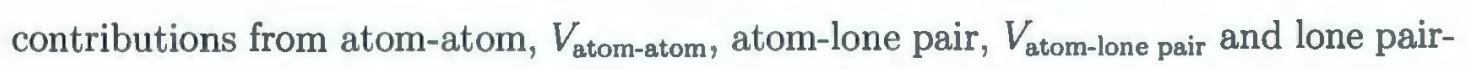




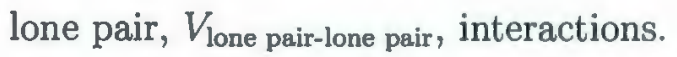

$$
V(\mathbf{R})=V_{\text {atomic }}+V_{\text {atom-atom }}+V_{\text {atom-lone pair }}+V_{\text {lone pair-lone pair }}
$$

The electronic kinetic energy is included through the virial theorem (see discussion at end of this section). Each energy term is further subdivided into electron-electron, electron-nuclear, and nuclear-nuclear potential energy, $V=V^{e e}+V^{N e}+V^{N N}$.

The atomic energy, which does not depend on the nuclear coordinates, accounts for the energy associated with atoms and their own electrons. Each atom has its own electrons corresponding to the neutral atom. The electronic configuration of each atom follows a Lewis dot structure approach. For example, an $\mathrm{sp}^{3}$ carbon atom has four unpaired valence electrons, and a pair of core electrons. Also, an $\mathrm{sp}^{3}$ nitrogen atom has three unpaired valence electrons, a lone pair, and a pair of core electrons. The atomic energy term is calculated as a sum over the contributions of individual nuclei, $V_{\text {atomic }}=\sum_{A=1}^{M} V_{A}$, where $M$ is the total number of nuclei.

$$
\begin{aligned}
V_{A}=\sum_{a \in A} 2 V_{A a}^{N e}+ & \sum_{i \in A} V_{A i}^{N e}+\sum_{a \in A}\left\{\sum_{\substack{b \in A \\
b \leq a}}\left(2-\delta_{a b}\right) V_{a b}^{e e}+\sum_{i \in A} V_{a i}^{e e}\right\} \\
& +\sum_{i \in A} \sum_{\substack{j \in A \\
j<i}} \frac{1}{2} V_{i j}^{e e}+\sum_{\mu \in A} V_{\mu \mu}^{e e},
\end{aligned}
$$

where $a$ and $b$ are pairs of core electrons, $i$ and $j$ are valence electrons, and $\mu$ is a lone pair. Each atom has nuclear-electron potential energy associated with its nucleus, $A$, and its electrons, $V_{A a}^{N e}$ and $V_{A i}^{N e}$. Each atom also has electron-electron potential energy due to its electrons, $V_{a b}^{e e}, V_{a i}^{e e}$, and $V_{i j}^{e e}$, as well as the potential energy of the electrons in each of its lone pairs, $V_{\mu \mu}^{e e}$. The potential energy, $V_{\mu \mu}^{e e}$, due to each lone pair is included in the atomic energy because, like the atomic energy, it is distance independent. 
The distance dependent energy terms include $V_{\text {atom-atom }}, V_{\text {atom-lone pair }}$, and $V_{\text {lone pair-lone pair }}$. The atom-atom interaction energy is the sum of the interactions between each atom

$$
\begin{gathered}
\text { pair, } V_{\text {atom-atom }}=\sum_{A=1}^{M} \sum_{B<A} V_{A B}\left(R_{A B}\right) . \\
V_{A B}\left(R_{A B}\right)=\frac{Z_{A} Z_{B}}{R_{A B}}+\sum_{a \in A} 2 V_{B a}^{N e}\left(R_{A B}\right)+\sum_{a \in B} 2 V_{A a}^{N e}\left(R_{A B}\right) \\
+\sum_{i \in A} V_{B i}^{N e}\left(R_{A B}\right)+\sum_{i \in B} V_{A i}^{N e}\left(R_{A B}\right)+\sum_{a \in A}\left\{\sum_{b \in B} 2 V_{a b}^{e e}\left(R_{A B}\right)+\sum_{i \in B} V_{a i}^{e e}\left(R_{A B}\right)\right\} \\
+\sum_{b \in B} \sum_{i \in A} V_{b i}^{e e}\left(R_{A B}\right)+\sum_{\substack{i \in A \\
(i \in c)}}\left\{\sum_{\substack{j \in B \\
(j \notin c)}} \frac{1}{2} V_{i j}^{e e}\left(R_{A B}\right)+\sum_{\substack{j \in B \\
(j \in c)}} V_{i j}^{e e}\left(R_{A B}\right)\right\},
\end{gathered}
$$

where $c$ denotes a bond between atoms $A$ and $B$. This interatomic energy includes core electron pairs and valence electrons of one atom attracted to another, $V_{B a}^{N e}$, $V_{A a}^{N e}, V_{B i}^{N e}$ and $V_{A i}^{N e}$, and potential energy between electrons of different atoms, $V_{a b}^{e e}$, $V_{a i}^{e e}, V_{b i}^{e e}$ and $V_{i j}^{e e}$. The atom-lone pair interaction energy is given by, $V_{\text {atom-lone pair }}=$ $\sum_{A=1}^{M} \sum_{\mu=1}^{L} V_{A \mu}\left(R_{A \mu}\right)$, where $L$ is the total number of lone pairs, and

$$
V_{A \mu}\left(R_{A \mu}\right)=2 V_{A \mu}^{N e}\left(R_{A \mu}\right)+\sum_{a \in A} 2 V_{a \mu}^{e e}\left(R_{A \mu}\right)+\sum_{i \in A} V_{i \mu}^{e e}\left(R_{A \mu}\right)
$$

There is nuclear attraction potential energy between atom $A$ and lone pair $\mu, V_{A \mu}^{N e}$, and electron-electron potential energy, $V_{a \mu}^{e e}$ and $V_{i \mu}^{e e}$. The lone pair-lone pair interaction energy consists of only electron-electron potential energy, where $V_{\text {lone pair-lone pair }}=$ $\sum_{\mu=1}^{L} \sum_{\nu<\mu} V_{\mu \nu}$

$$
V_{\mu \nu}=2 V_{\mu \nu}^{e e}\left(R_{\mu \nu}\right)
$$

This formulation of simulated electronic structure theory contains no electronic kinetic energy terms. It was found that kinetic energy terms were not required to accurately model the systems studied thus far. However, kinetic energy, like electronnucleus potential energy (Section 1.2), correlates quite well with the average distance 
between an electron and a nucleus. Therefore, it is possible to include terms to model kinetic energy in future development of the theory if desired. In order to calculate the energy of a molecular system, $E(\mathbf{R})$, without the kinetic energy of the electrons, it is assumed that the virial coefficient, which is the negative ratio of the potential and kinetic energy, $c_{v}=-V / T$, is constant over the entire potential energy surface.

$$
E(\mathbf{R})=\frac{\left(c_{v}-1\right)}{c_{v}} V(\mathbf{R})
$$

In this study, the equilibrium geometries and energies of the molecules were fit to RHF/6-31G(d) values, therefore the virial coefficient is set to the value obtained from the equilibrium geometry at $\mathrm{RHF} / 6-31 \mathrm{G}(\mathrm{d})$.

\subsection{The average interparticle distance and modelling molecular orbital en- ergy components}

Simulated electronic structure theory models the PESs of molecular systems in which the energy contribution of electron pairs and individual electrons are functions of the nuclear coordinates. It is useful to obtain parameters for these models, as well as insight on how the energy contributions per electron pair and individual electron depend on molecular geometry, from $a b$ initio models of electronic structure. Such properties are easily examined at the Hartree-Fock level of theory. The closed-shell Hartree-Fock (RHF) energy is given as

$$
E_{\mathrm{HF}}=\sum_{a=1}^{N / 2} 2 h_{a}+\sum_{a=1}^{N / 2} \sum_{b=1}^{N / 2} 2 J_{a b}-K_{a b}
$$

where $N$ is the number of electrons, $h_{a}$ is the one-electron energy, $J_{a b}$ is the Coulomb energy, and $K_{a b}$ is the exchange energy, associated with molecular orbitals (MOs) $a$ 
and $b$. The one-electron energy, $h_{a}=T_{a}+V_{a}$, consists of kinetic energy,

$$
T_{a}=\left\langle a\left|-\frac{1}{2} \nabla_{1}^{2}\right| a\right\rangle
$$

and nuclear-electron attraction potential energy,

$$
V_{a}=\left\langle a\left|-\sum_{A=1}^{M} \frac{Z_{A}}{r_{1 A}}\right| a\right\rangle .
$$

The Coulomb and exchange energy are calculated from two-electron integrals.

$$
\begin{gathered}
J_{a b}=\langle a b \mid a b\rangle \\
K_{a b}=\langle a b \mid b a\rangle
\end{gathered}
$$

In recent work, ${ }^{7}$ it was found that the Coulomb energy could be modelled with a one-electron property of MOs, the average interelectronic distance, $\delta r_{12}$. The Coulomb energy, $J_{a b}$, associated with any two MOs is inversely proportional to the average distance between an electron in MO $a$ and an electron in MO b, $\left(\delta r_{12}\right)_{a b}$, which is given by

$$
\left(\delta r_{12}\right)_{a b}=\sqrt{\left\langle a b\left|r_{12}^{2}\right| a b\right\rangle}
$$

The relationship,

$$
J_{a b} \approx \frac{\alpha}{\left(\delta r_{12}\right)_{a b}}
$$

has severe deviations for the canonical MOs (CMOs) of HF theory, due to delocalized core MO pairs. However, if localized MOs (LMOs) are used the relationship is followed quite closely, Figure 5.1.

A similar relationship has been found between the nuclear-electron attraction potential energy, $V_{a}$ (Equation 5.11), and the average distance between an electron 


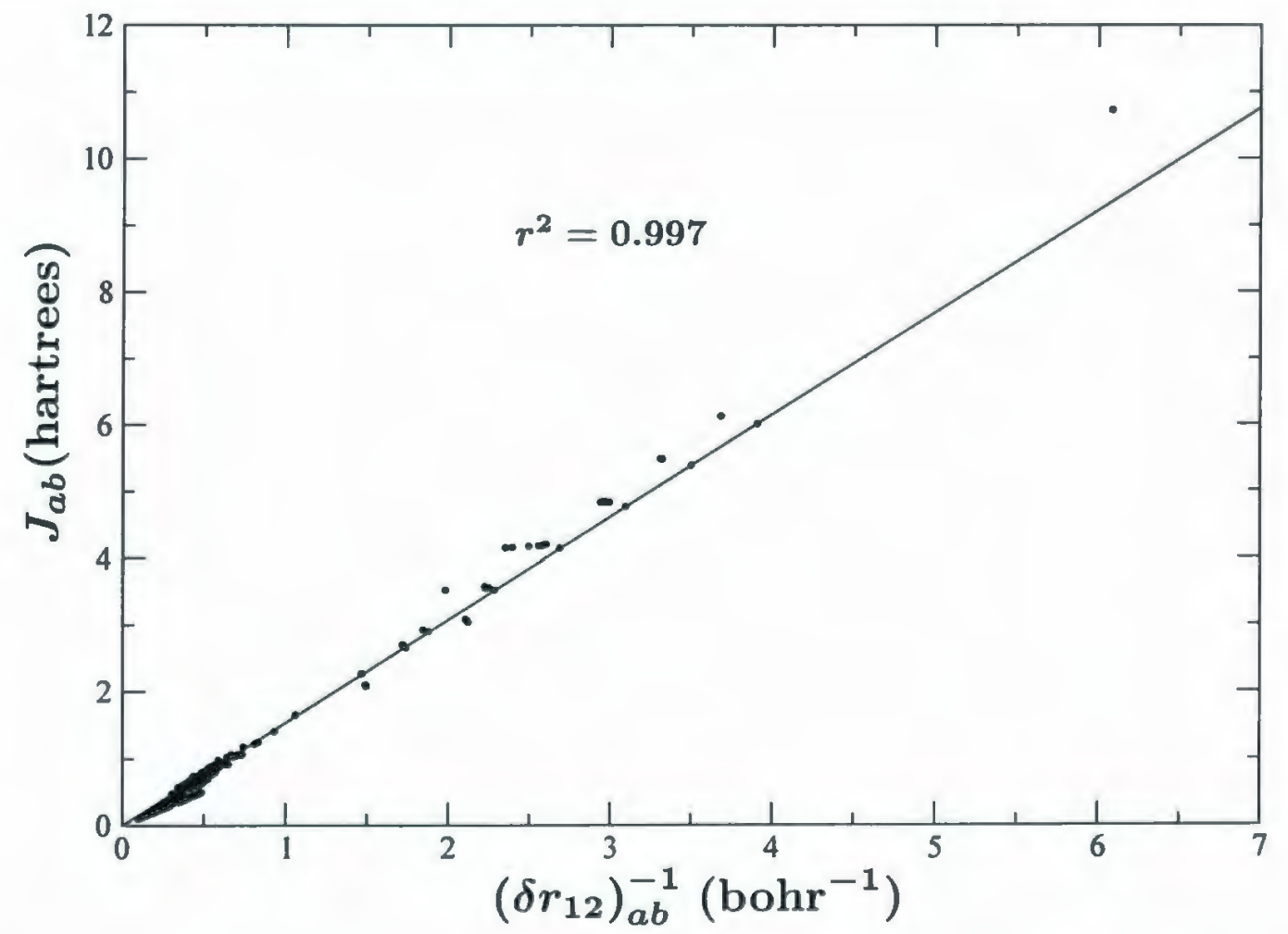

Figure 5.1. Relationship between Coulomb energy, $J_{a b}$, and the average distance between two electrons, $\left(\delta r_{12}\right)_{a b}$, for two electron ions to molecules with up to 58 electrons (HF/6-31G(d), LMO). From reference 7 . 
and a nucleus, $\delta r_{a A}$, which is calculated analogously to $\left(\delta r_{12}\right)_{a b \cdot}{ }^{8}$

$$
\delta r_{a A}=\sqrt{\left\langle a\left|r_{1 A}^{2}\right| a\right\rangle}
$$

The nuclear-electron potential energy, $V_{a}$, may be separated into contributions from individual nuclei, $V_{a A}$, where $\sum_{A=1}^{M} V_{a A}=V_{a}$. The nuclear-electron potential energy between MO $a$ and nucleus $A$, is related to $\delta r_{a A}$ through the equation

$$
V_{a A} \approx-\gamma \frac{Z_{A}}{\delta r_{a A}}
$$

The relationship has the same deviations for CMOs as seen with $J_{a b}$ and $\left(\delta r_{12}\right)_{a b}$, and performs quite well for LMOs, Figure 5.2. It should be noted that while the overall correlation in Figures 5.1 and 5.2 is good, $r^{2}=0.997$ and 0.995 respectively, individual deviations approach 1 hartree for the relationship between $J_{a b}$ and $\left(\delta r_{12}\right)_{a b}$ and 25 hartrees for $V_{a A}$ and $\delta r_{a A}$. These errors are quite large on the scale of reaction energetics. However, these deviations involve core MOs, which correspond to the core electron pairs of SEST, for which the energy terms are constant parameters and therefore do not depend upon the above relationships. Furthermore, it is the qualitative result which is most relevant to an empirically parametrized SEST. The Coulomb and electron-nuclear potential energy are quantities calculated from integrating over the electronic distribution. Although, as a result of the relationships just mentioned, to some approximation, electrons have average positions with respect to each other and with respect to the nuclei. Furthermore, these average positions and hence distances may be used to approximate components of the HF energy per MO. An immediate question following such deductions is, how do these average interparticle distances change with changing molecular structure? 


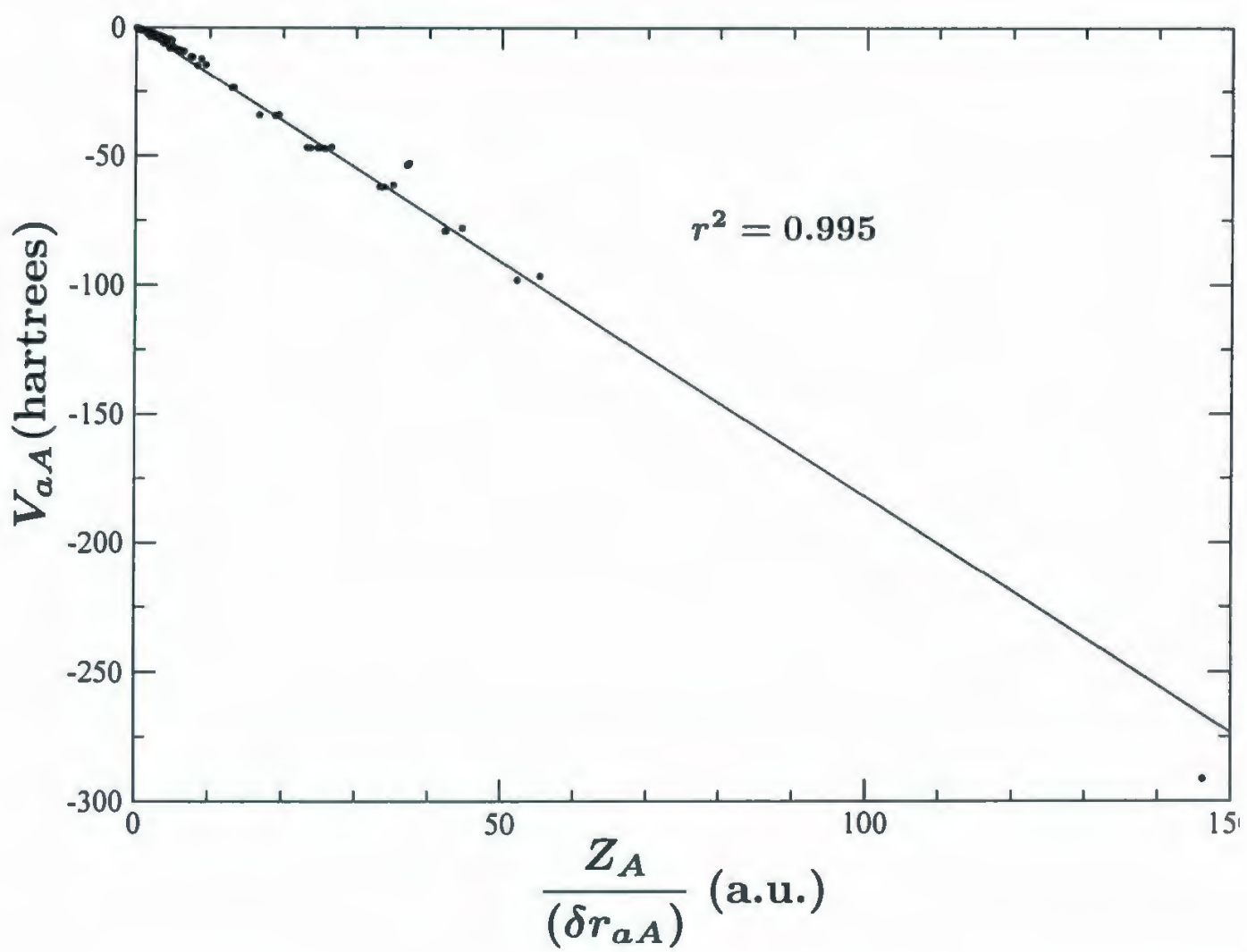

Figure 5.2. Relationship between electron-nuclear attraction energy, $V_{a A}$, and the average distance between an electron and a nucleus, $\delta r_{a A}$, for two electron ions to molecules with up to 58 electrons (HF/6-31G(d), LMO). 
In general, the average interparticle distances, $\left(\delta r_{12}\right)_{a b}$ and $\delta r_{a A}$, are linearly related to interatomic distance. This relationship is illustrated in Figures 5.3 and 5.4, with $F_{2}$. It is expected that such a relationship would be most critical for atoms

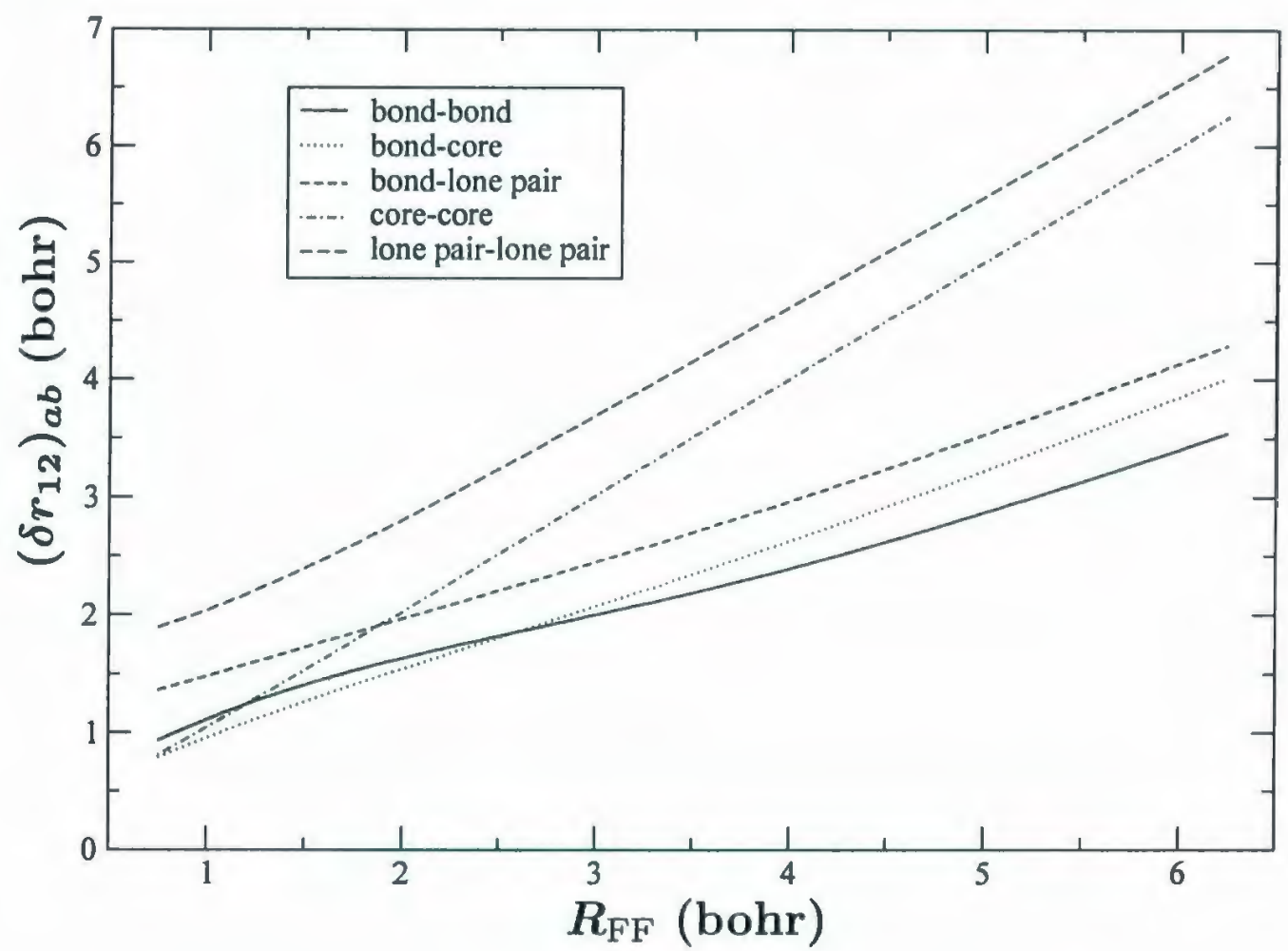

Figure 5.3. Relationship between the average distance between two electrons, $\left(\delta r_{12}\right)_{a b}$, and the fluorine-fluorine distance, $R_{\mathrm{FF}}$, in $\mathrm{F}_{2}$ (HF/6-31G(d), LMO). Includes $a=b=$ bond, $a=$ bond $b=$ core, $a=$ core $b=$ core, and $a=$ lone pair $b=$ lone pair(from separate nuclei).

which are bonded to each other. Changing bond length significantly affects the electronic structure of a molecule, especially around the two bonded atoms, and even more for a diatomic. While this may be the case, it is seen in Figures 5.3 and 5.4 that the interparticle distances very closely follow a linear relationship with the fluorinefluorine distance. However, for shorter bond lengths ( $<1.5$ bohr), there is a slight 


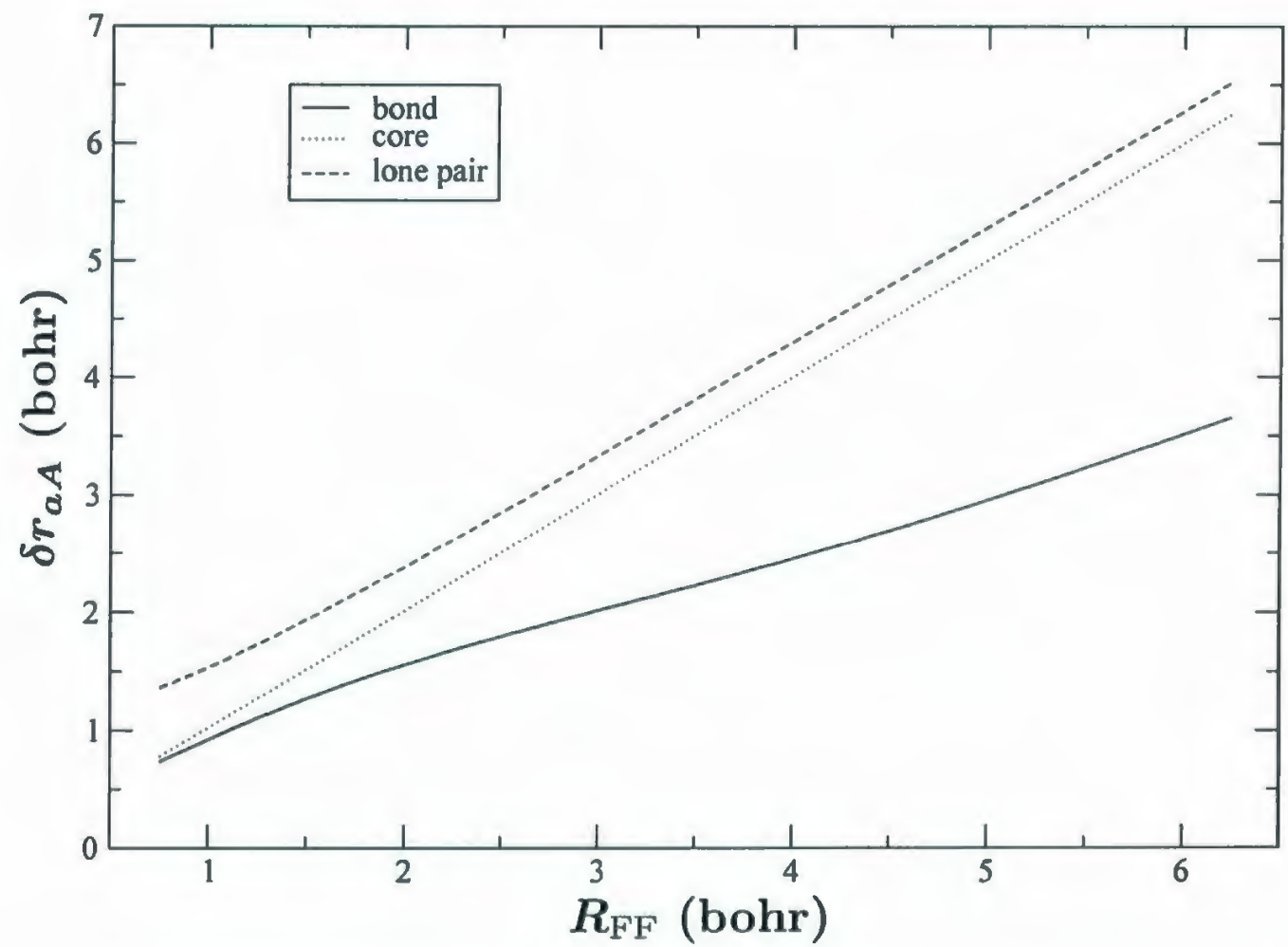

Figure 5.4. Relationship between the average distance an electron and a nucleus, $\delta r_{a A}$, and the fluorine-fluorine distance, $R_{\mathrm{FF}}$, in $\mathrm{F}_{2}$ (HF/6-31G(d), LMO). Includes $a=$ bond, core, and lone pair. 
curvature in the lone pair-lone pair $\left(\delta r_{12}\right)_{a b}$ and lone pair $\delta r_{a A}$ curves which is due to the lone pairs on different fluorine atoms bending away from each other as the bond length decreases. It is also observed in both figures, that distances involving electrons in the bond show slight curvature. In both the bond-bond $\left(\delta r_{12}\right)_{a b}$ and bond $\delta r_{a A}$ curves, the slope is less around the equilibrium bond length $\left(\left(R_{\mathrm{FF}}\right)_{e}=2.54 \mathrm{bohr}\right)$ than that at the shorter and longer $R_{F F}$ distances. Nonetheless, this relationship, even as an approximation, is a powerful tool.

The ability to relate the average interparticle distances to atomic distances has important consequences. For a given molecular structure, not only will these relationships provide an estimate of MO energy components, they will provide a means of predicting an average picture of the electronic structure.

\subsection{Functional Form}

It was seen in section 1.1 that the SEST energy expression, Equation 5.3, contains terms which depend on atom-atom, atom-lone pair and lone pair-lone pair distances. The functional form of these distance dependent terms determines the success of applying SEST. Following the relationships observed in Figures 5.3 and 5.4, the interparticle distances, $\left(\delta r_{12}\right)_{a b}$ and $\delta r_{b A}$, are taken to be linear functions of the interatomic distance, $R_{A B}$.

$$
\left(\delta r_{12}\right)_{a b}=\lambda R_{A B}+\sigma
$$

and

$$
\delta r_{b A}=\omega R_{A B}+\kappa
$$

where $\mathrm{MO} a$ is localized on atom $A, \mathrm{MO} b$ is localized on atom $B$, or one of them ( $a$ or $b$ ) is the bond between $A$ and $B$, and $\lambda, \sigma, \omega$, and $\kappa$ are constants for each unique pair 
of MOs. As observed in Figures 5.3 and 5.4, the relationships involving bonding MOs is approximate. However, an exact model of $a b$ initio electronic structure through interatomic distances is not the goal, neither is it possible.

The LMO potential energy components are related to the interatomic distance through the linear relationships above and the relationships seen earlier (Figures 5.1 and 5.2). Substitution of Equation 5.18 into Equation 5.15 yields

$$
J_{a b} \approx \frac{\alpha}{\lambda R_{A B}+\sigma}
$$

while substitution of Equation 5.19 into Equation 5.17 gives

$$
V_{b A} \approx \frac{-\gamma Z_{A}}{\omega R_{A B}+\kappa}
$$

The above equations are used to derive the general form of the SEST distance dependent functions.

Given electron pairs $a$ and $b$, where $a$ is localized on atom $A$ and $b$ is localized on atom $B$, the electron-electron potential energy between the two pairs of electrons is given by

$$
V_{a b}^{e e}\left(R_{A B}\right)=\frac{\left(V_{a b}^{e e}\right)_{e}\left(\left(R_{A B}\right)_{e}-r_{a b}^{e e}\right)}{R_{A B}-r_{a b}^{e e}}
$$

where $\left(R_{A B}\right)_{e}$ is an equilibrium interatomic distance, $r_{a b}^{e e}$ is a constant parameter, and $\left(V_{a b}^{e e}\right)_{e}$ is the potential energy at the equilibrium distance. Also, as the interatomic distance, $R_{A B}$, approaches infinity, the potential energy term vanishes. The SEST electron-electron potential energy function is chosen to model the total HF electronelectron potential energy (Equation 5.9),

$$
V_{a b}^{e e}\left(R_{A B}\right) \approx 2 J_{a b}-K_{a b}
$$


although it is only the Coulomb energy, $J_{a b}$, which is inversely proportional to the average interelectronic distance, $\left(\delta r_{12}\right)_{a b}$ (Equation 5.15). The functional form is derived using localized molecular orbitals (LMOs), ${ }^{9}$ in which case the exchange energy, $K_{a b}$, is minimal. Therefore the approximation in Equation 5.23 is considered valid. The electron-nucleus potential energy is calculated in a similar fashion.

$$
V_{B a}^{N e}\left(R_{A B}\right)=\frac{\left(V_{B a}^{N e}\right)_{e}\left(\left(R_{A B}\right)_{e}-r_{B a}^{N e}\right)}{R_{A B}-r_{B a}^{N e}}
$$

While the functions given in Equations 5.22 and 5.24 are for pairs of electrons, $a$ and $b$, functions for individual electrons, $i$ and $j$, and any combination of pairs and individual electrons (eg. $V_{a i}^{e e}\left(R_{A B}\right)$ ) take the same form, albeit with different parameter values. The parameters of these functions are derived from the LMOs of the ab initio electronic structure of a molecule.

It is recognized that positive values of the parameters $r_{a b}^{e e}, r_{B a}^{N e}$, etc., result in singularities in the potential energy surface for positive values of $R_{A B}$. While fitting a potential energy surface, parameters with values larger than $\frac{1}{2}\left(R_{A B}\right)_{e}$ are avoided, and values smaller than this threshold are dealt with using a piecewise function.

$$
\begin{aligned}
& V_{A B}\left(R_{A B}\right)=\left\{\begin{aligned}
V^{N N}\left(R_{A B}\right)+V^{N e}\left(R_{A B}\right)+V^{e e}\left(R_{A B}\right) & , \text { if } R_{A B}>r_{A B}^{\max }+\delta \\
V^{N N}\left(R_{A B}\right)+V^{N e}\left(r_{A B}^{\max }+\delta\right)+V^{e e}\left(r_{A B}^{\max }+\delta\right) & , \text { if } R_{A B} \leq r_{A B}^{\max }+\delta
\end{aligned}\right. \\
& V_{A \mu}\left(R_{A \mu}\right)=\left\{\begin{aligned}
V^{N e}\left(R_{A \mu}\right)+V^{e e}\left(R_{A \mu}\right) & \text {, if } R_{A \mu}>r_{A \mu}^{\max }+\delta \\
V^{N e}\left(r_{A \mu}^{\max }+\delta\right)+V^{e e}\left(r_{A \mu}^{\max }+\delta\right) & \text {, if } R_{A \mu} \leq r_{A \mu}^{\max }+\delta \\
+V^{e e}\left(R_{A \mu}+r_{i}\right)-V^{e e}\left(r_{A \mu}^{\max }+\delta+r_{i}\right) &
\end{aligned}\right.
\end{aligned}
$$

The largest positive value for a given atom-atom interaction or atom-lone pair interaction is $r^{\max }$ (eg. $\left.r_{A B}^{\max }=\max \left\{r_{A i}^{N e}, r_{B a}^{N e}, r_{i j}^{e e}, \ldots\right\}\right)$ ). The argument $R_{A \mu}+r_{i}$ 
(Equation 5.26) denotes that for each given function of the form in Equation 5.22 and 5.24 , the value of the specific $r_{i}$ parameter is added to $R_{A \mu}$, thus removing $r_{i}$ from the denominator.

\subsection{Determining the Parameters}

In this study, the parameters of the distance dependent functions (Equations 5.22 and 5.24) along with the constant energy terms (Equation 5.4) are derived from $a b$ initio calculations. The LMO energy components, $V_{a A}, J_{a b}$, and $K_{a b}$ are used to define the constant energy terms, $V_{A}$, and the equilibrium energy terms, $\left(V_{A B}\right)_{e},\left(V_{A \mu}\right)_{e}$, and $\left(V_{\mu \nu}\right)_{e}$. For electron pairs belonging to the same atom (core electrons) or a lone pair, the energy is taken to be equal to the corresponding LMO energy component. For example;

$$
\left(V_{a b}^{e e}\right)_{e}=2 J_{a b}^{\mathrm{LMO}}-K_{a b}^{\mathrm{LMO}}
$$

and

$$
V_{A a}^{N e}=V_{a A}^{\mathrm{LMO}}
$$

Energy terms for individual electrons, the bonding electrons, requires the partitioning of LMO energy components. The energy expression for ammonia may be used to illustrate an energy partitioning scheme.

$$
V_{\mathrm{NH}_{3}}=V_{\mathrm{N}}+3 V_{\mathrm{H}}+3 V_{\mathrm{NH}}+3 V_{\mathrm{HH}}+V_{\mathrm{N} \mu}+3 V_{\mathrm{H} \mu}
$$

The lone pair of nitrogen is denoted $\mu$. The terms $V_{N}$ and $V_{H}$ are the atomic energies of nitrogen and hydrogen, respectively.

$$
V_{\mathrm{N}}=2 V_{\mathrm{N} \alpha}^{N e}+3 V_{\mathrm{N} i}^{N e}+V_{a a}^{e e}+3 V_{a i}^{e e}+3\left(\frac{1}{2} V_{i j}^{e e}\right)+V_{\mu \mu}^{e e}
$$


where $a$ is the pair of core electrons of nitrogen and, $i$ and $j$ denote valence electrons of nitrogen.

$$
V_{\mathrm{H}}=V_{\mathrm{H} k}^{N e},
$$

where $k$ is the hydrogen electron. The atom-atom interaction energy terms are $V_{\mathrm{NH}}$ and $V_{\mathrm{HH}}$.

$$
\begin{aligned}
V_{\mathrm{NH}}=\frac{Z_{\mathrm{N}} Z_{\mathrm{H}}}{R_{\mathrm{NH}}} & +2 V_{\mathrm{H} a}^{N e}\left(R_{\mathrm{NH}}\right)+2 V_{\mathrm{H} j}^{N e}\left(R_{\mathrm{NH}}\right)+V_{\mathrm{H} i}^{N e}\left(R_{\mathrm{NH}}\right)+V_{\mathrm{N} k}^{N e}\left(R_{\mathrm{NH}}\right) \\
& +V_{a k}^{e e}\left(R_{\mathrm{NH}}\right)+2\left(\frac{1}{2} V_{j k}^{e e}\left(R_{\mathrm{NH}}\right)\right)+V_{i k}^{e e}\left(R_{\mathrm{NH}}\right)
\end{aligned}
$$

where $a$ is the pair of core electrons of nitrogen, $i$ is the valence electron of nitrogen in this NH bond, $j$ is a valence electron of nitrogen not in this bond, and $k$ is the hydrogen electron. The hydrogen-hydrogen non-bonded interaction energy is described as

$$
V_{\mathrm{HH}}=\frac{Z_{\mathrm{H}} Z_{\mathrm{H}}}{R_{\mathrm{HH}}}+2 V_{\mathrm{Hi}}^{N e}\left(R_{\mathrm{HH}}\right)+\frac{1}{2} V_{i j}^{e e}\left(R_{\mathrm{HH}}\right) .
$$

Finally, $V_{\mathrm{N} \mu}$ and $V_{\mathrm{H} \mu}$ are the atom-lone pair interaction terms.

$$
\begin{gathered}
V_{\mathrm{N} \mu}=2 V_{\mathrm{N} \mu}^{N e}\left(R_{\mathrm{N} \mu}\right)+2 V_{a \mu}^{e e}\left(R_{\mathrm{N} \mu}\right)+3 V_{i \mu}^{e e}\left(R_{\mathrm{N} \mu}\right) \\
V_{\mathrm{H} \mu}=2 V_{\mathrm{H} \mu}^{N e}\left(R_{\mathrm{H} \mu}\right)+V_{k \mu}^{e e}\left(R_{\mathrm{H} \mu}\right)
\end{gathered}
$$

The derivation of the parameters for the electron-nucleus and electron-electron potential energy terms from LMO energy values is given in Tables 5.1 and 5.2, respectively.

Similar schemes are used to partition the energies of the bonding electrons of other molecules. In some cases, there may be a point in which the partition is arbitrary and is defined intuitively (see $V_{a i}^{e e}$ and $\left(V_{i \mu}^{e e}\right)_{e}$ in Table 5.2). Also, the partitioning of the energy for $V_{\mathrm{Ni}}^{\mathrm{Ne}}$ (Table 5.1) and $V_{d i}^{e e}$ (Table 5.2) ensures that dissociation of a single 
TABLE 5.1: Derivation of SEST electron-nucleus potential energy parameters for ammonia from RHF/6-31G(d) LMO energy components. ${ }^{a}$

\begin{tabular}{|c|c|c|}
\hline Parameter & Equation & Derived from ${ }^{b}$ \\
\hline$V_{\mathrm{Na}}^{\mathrm{Ne}}$ & 5.30 & $V_{a N}^{\text {LMO }}$ \\
\hline$\left(V_{\mathrm{Ha}}^{\mathrm{Ne}}\right)_{e}$ & 5.32 & $V_{a H}^{\text {LMO }}$ \\
\hline$\left(V_{\mathbf{N} \mu}^{N e}\right)_{e}$ & 5.34 & $V_{\mu \mathrm{N}}^{\mathrm{LMO}}$ \\
\hline$\left(V_{\mathbf{H} \mu}^{N e}\right)_{e}$ & 5.35 & $V_{\mu \mathrm{H}}^{\mathrm{LMO}}$ \\
\hline$\left(V_{\mathrm{H}_{j}}^{N e}\right)_{e}$ & 5.32 & $2 V_{\mathrm{dH}}^{\mathrm{LMO}}-V_{\mathrm{Hi}}^{\mathrm{Ne}}\left(R_{\mathrm{HH}}\right)^{c}$ \\
\hline$V_{\mathrm{Ni}}^{\mathrm{Ne}}$ & 5.30 & $V_{\mathrm{NH}_{2}}^{\mathrm{Ne}}-2\left(V_{\mathrm{Na}}^{\mathrm{Ne}}+2\left(V_{\mathrm{Ha}}^{\mathrm{Ne}}\right) e+2 V_{\mathrm{cN}}^{L M O}+2 V_{c \mathrm{H}}^{L M O}\right.$ \\
\hline & & $\left.+2 V_{d \mathbf{H}}^{L M O}+\left(V_{\mathrm{N} \mu}^{N e}\right)_{e}+2\left(V_{\mathrm{H} \mu}^{N e}\right)_{e}\right)-2\left(V_{\mathrm{H} j}^{N e}\right)_{e}{ }^{d}$ \\
\hline$\left(V_{\mathrm{N} k}^{N e}\right)_{e}$ & 5.32 & $2 V_{\mathrm{cN}}^{\mathrm{LMO}}-V_{\mathrm{Ni}}^{\mathrm{Ne}}$ \\
\hline$\left(V_{\mathrm{Hi}}^{N e}\right)_{e}$ & 5.32 & $2 V_{c \mathrm{H}}^{\mathrm{LMO}}-V_{\mathrm{Hk}}^{\mathrm{Ne}}$ \\
\hline
\end{tabular}

${ }^{a} \mu$ denotes the lone pair of nitrogen, $a$ denotes the pair of core electrons of nitrogen

$c$ denotes a bond involving the specified atom

$d$ denotes a bond not involving specified atom (i.e. another bond)

$i$ and $j$ denote valence electrons of nitrogen, $k$ denotes electron of hydrogen.

${ }^{b}$ Terms of the form $V^{\text {LMO }}$ denote energy values taken from LMOs of an $a b$ initio calculation.

${ }^{c} V_{\mathrm{Hi}}^{N e}\left(R_{\mathrm{HH}}\right)$ denotes electron-nucleus potential energy of previously defined HH non-bonded interaction, calculated at the equilibrium ammonia $\mathrm{HH}$ distance, $R_{\mathrm{HH}}$.

${ }^{d} V_{\mathrm{NH}_{2}}^{\mathrm{Ne}_{2}}$ denotes total electron-nucleus potential energy of the radical amine calculated at UHF/6-31G(d). 
TABLE 5.2: Derivation of SEST electron-electron potential energy parameters for ammonia from UHF/6-31G(d) LMO energy components. ${ }^{a}$

\begin{tabular}{|c|c|c|}
\hline Parameter & Equation & Derived from ${ }^{b}$ \\
\hline$V_{a a}^{e e}$ & 5.30 & $V_{a a}^{\text {LMO }}$ \\
\hline$V_{\mu \mu}^{e e}$ & 5.30 & $V_{\mu \mu}^{\mathrm{LMO}}$ \\
\hline$\left(V_{a \mu}^{e e}\right)_{e}$ & 5.34 & $V_{a \mu}^{\mathrm{LMO}}$ \\
\hline$V_{d i}^{e e}$ & intermediate term ${ }^{c}$ & $\left\{V_{\mathrm{NH}_{2}}^{e e}-V_{a a}^{e e}-2 V_{c c}^{L M O}-2 V_{c d}^{L M O}-4 V_{c a}^{L M O}-V_{\mu \mu}^{e e}\right.$ \\
\hline & & $\left.-4 V_{c \mu}^{L M O}-2\left(V_{a \mu}^{e e}\right) e\right\}\left(2+\left(\frac{V_{c q}^{L M O}}{V_{c d}^{L H O O}}\right)+\left(\frac{V_{c \mu}^{L M O}}{V_{c d}^{L M O}}\right)\right)^{-1} d$ \\
\hline$V_{a i}^{e e}$ & 5.30 & $V_{d i}^{e e}\left(\frac{V_{q d}^{L M O}}{V_{c d}^{L M O}}\right)$ \\
\hline$\left(V_{i \mu}^{e e}\right)_{e}$ & 5.34 & $V_{d i}^{e e}\left(\frac{V_{c k}^{L M O}}{V_{c d}^{L A N O}}\right)$ \\
\hline$\left(V_{k \mu}^{e e}\right)_{e}$ & 5.35 & $2 V_{c \mu}^{\mathrm{LMO}}-\left(V_{i \mu}^{e e}\right)_{e}$ \\
\hline$\left(V_{a k}^{e e}\right)_{e}$ & 5.32 & $2 V_{c a}^{\text {LMO }}-V_{a i}^{e e}$ \\
\hline$V_{d k}^{e e}$ & intermediate term ${ }^{c}$ & $2 V_{c d}^{\mathrm{LMO}}-V_{d i}^{e e}$ \\
\hline$\left(V_{j k}^{e e}\right)_{e}$ & 5.32 & $2 V_{d k e}^{e e}-V_{i j}^{e e}\left(R_{\mathrm{HH}}\right)^{e}$ \\
\hline$V_{i j}^{e e}$ & 5.30 & $2 V_{d i}^{e e}-\left(V_{j k}^{e e}\right)$ \\
\hline$\left(V_{i k}^{e e}\right)_{e}$ & 5.32 & $V_{c c}^{\text {LMO }}$ \\
\hline
\end{tabular}

${ }^{a} \mu$ denotes the lone pair of nitrogen, $a$ denotes the pair of core electrons of nitrogen

$c$ denotes a bond involving the specified electron

$d$ denotes a bond not involving the specified electron (i.e. another bond)

$i$ and $j$ denote valence electrons of nitrogen, $k$ denotes electron of hydrogen.

${ }^{b}$ Terms of the form $V^{\text {LMO }}$ denote energy values taken from LMOs of an $a b$ initio calculation.

(where $V_{a b}^{\text {LMO }}=2 J_{a b}^{\text {LMO }}-K_{a b}^{\text {LMO }}$ )

'Intermediate terms used to simplify expressions for some energy parameters.

${ }^{d} V_{\mathrm{NH}_{2}}^{e e}$ denotes total electron-electron potential energy of the radical amine calculated at UHF/6-31G(d).

${ }^{e} V_{i j}^{e e}\left(R_{\mathrm{HH}}\right)$ denotes electron-electron potential energy of previously defined HH non-bonded interaction, calculated at the equilibrium ammonia $\mathrm{HH}$ distance, $R_{\mathrm{HH}}$. 
bond leads to the UHF/6-31G(d) potential energy of the dissociated products. It is important to note that, for the equilibrium structure, the $\mathrm{MO}$ energy components, $V_{a A}^{L M O}$ and $V_{a b}^{L M O}$, can be recovered exactly. For example;

$$
V_{c d}^{L M O}=\frac{1}{2} V_{i j}^{e e}+2\left(\frac{1}{2} V_{i k}^{e e}\left(R_{\mathrm{NH}}\right)\right)+V_{k l}^{e e}\left(R_{\mathrm{HH}}\right),
$$

where $i$ and $j$ denote bonding electrons of nitrogen, and $k$ and $l$ are hydrogen electrons. Furthermore, for a distorted geometry, an approximation to the MO energy components may be calculated.

It is not surprising that a theory which parametrizes the energy of individual electrons and electron pairs derives the parameters from ab initio LMO calculations. SEST, an alternative approach to modelling PESs, shares the same premise of molecular mechanics, which is that certain functions and parameters are transferable from one molecule to another. The transferability of the properties of LMOs has been known for decades ${ }^{10,11}$ and work is currently being done to apply this transferability to linear scaling, divide and conquer methods. ${ }^{12-14}$

The other parameters of the distance dependent functions, $\left(R_{A B}\right)_{e}, r_{A B a}^{N e}, r_{A i B j}^{e e}$, etc., are defined through fitting of the equilibrium geometry, $\frac{\partial E\left(\mathbf{R}_{e}\right)}{\partial \mathrm{R}}=0$, and the vibrational frequencies.

\section{Method}

Simulated Electronic Structure Theory (SEST) was implemented within the MUNgauss program. ${ }^{15}$ The geometries of the molecules studied were optimized at RHF/6-31G(d) and the energies and MO properties were also calculated at RHF/6-31G(d). The energies of the products of bond dissociation were calculated at UHF/6-31G(d). Localized molecular orbitals were obtained through Boys localization. ${ }^{9}$ 


\section{Results and Discussion}

\subsection{Hydrogen molecule and the first-row hydrides}

An initial demonstration of SEST is given through modelling $a b$ initio calculations on small molecules, such as the hydrogen molecule and the first-row hydrides. The SEST energy expression for the hydrogen molecule is:

$$
V_{\mathrm{H}_{2}}\left(R_{\mathrm{HH}}\right)=2 V_{\mathrm{Hi}}^{N e}+\frac{Z_{\mathrm{H}} Z_{\mathrm{H}}}{R_{\mathrm{HH}}}+2 V_{\mathrm{HH} i}^{N e}\left(R_{\mathrm{HH}}\right)+V_{\mathrm{H} i \mathrm{H} j}^{e e}\left(R_{\mathrm{HH}}\right)
$$

The SEST model of $\mathrm{H}_{2}$, fits the RHF/6-31G(d) energy at the equilibrium bond

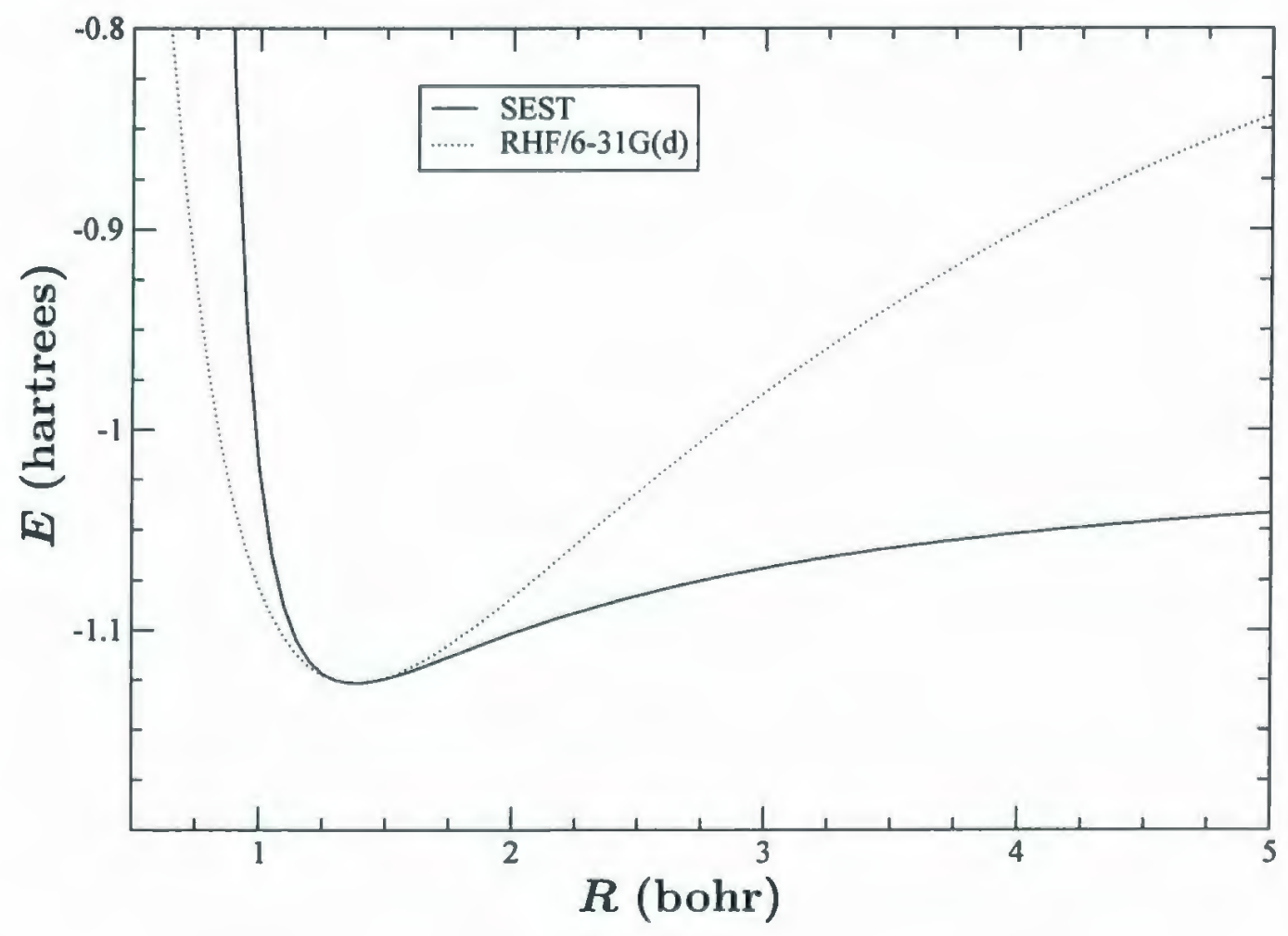

Figure 5.5. Dissociation curve for $\mathrm{H}_{2}$ (SEST and RHF/6-31G(d)).

length, $\left(R_{\mathrm{HH}}\right)_{e}=1.380$ bohr. At the dissociation limit, $R_{\mathrm{HH}} \rightarrow \infty$, the energy 
approaches the UHF/6-31G(d) potential energy of the dissociated products, $2 V_{\mathrm{H}}$, multiplied by the factor $\frac{c_{v}-1}{c_{v}}$, where $c_{v}$ is the virial coefficient of the equilibrium structure (Equation 5.8). The result is a dissociation energy which is 8 millihartrees smaller than the actual UHF/6-31G(d) dissociation energy. This could be corrected by first scaling the potential energy of the dissociated products $(2 \mathrm{H})$, before defining the energy parameters of the SEST model of $\mathrm{H}_{2}$. However, the purpose of the study is to illustrate the ability of the SEST model to qualitatively dissociate correctly (unlike RHF), the actual dissociation energy is adjustable. The SEST vibrational frequency of $\mathrm{H}_{2}$ is set to that of the RHF $/ 6-31 \mathrm{G}(\mathrm{d})$ calculation, $4646 \mathrm{~cm}^{-1}$. The hydrogen molecule energy expression (Equation 5.37) has only two parameters, $r_{\mathrm{HH} i}^{N e}$ and $r_{\mathrm{HiHj}}^{e e}$, which, in this case, are used to fit the first and second derivatives of the RHF /6-31G(d) wave function at the equilibrium bond length. The fit to equilibrium geometry and frequency is achieved by setting the parameters, $r_{\mathrm{HH} i}^{\mathrm{Ne}}$ and $r_{\mathrm{H} i \mathrm{H} j}^{e e}$, to $0.27291230 \mathrm{bohr}$ and 0.68583812 bohr, respectively. Similarly, for the first-row hydrides, SEST can model the HF equilibrium geometries, some frequencies, and allow for qualitatively correct dissociation of bonds.

Bond dissociation in the SEST models of the first-row hydrides resemble the potential energy curve of $\mathrm{H}_{2}$. In all cases, the equilibrium geometry and energy of the SEST model is identical to the RHF/6-31G(d) values and therefore these values are not reported. Also, for all systems, the dissociation of a single bond leads to the UHF/6-31G(d) potential energy of the dissociated products, multiplied by $\frac{c_{v}-1}{c_{v}}$. As previously mentioned, SEST provides an approximation to the energy components $2 J_{a b}-K_{a b}$ and $V_{a}$, for all MOs, for any point along the potential energy surface (dissociation curve). In this study, it is only the equilibrium values which have been fit 


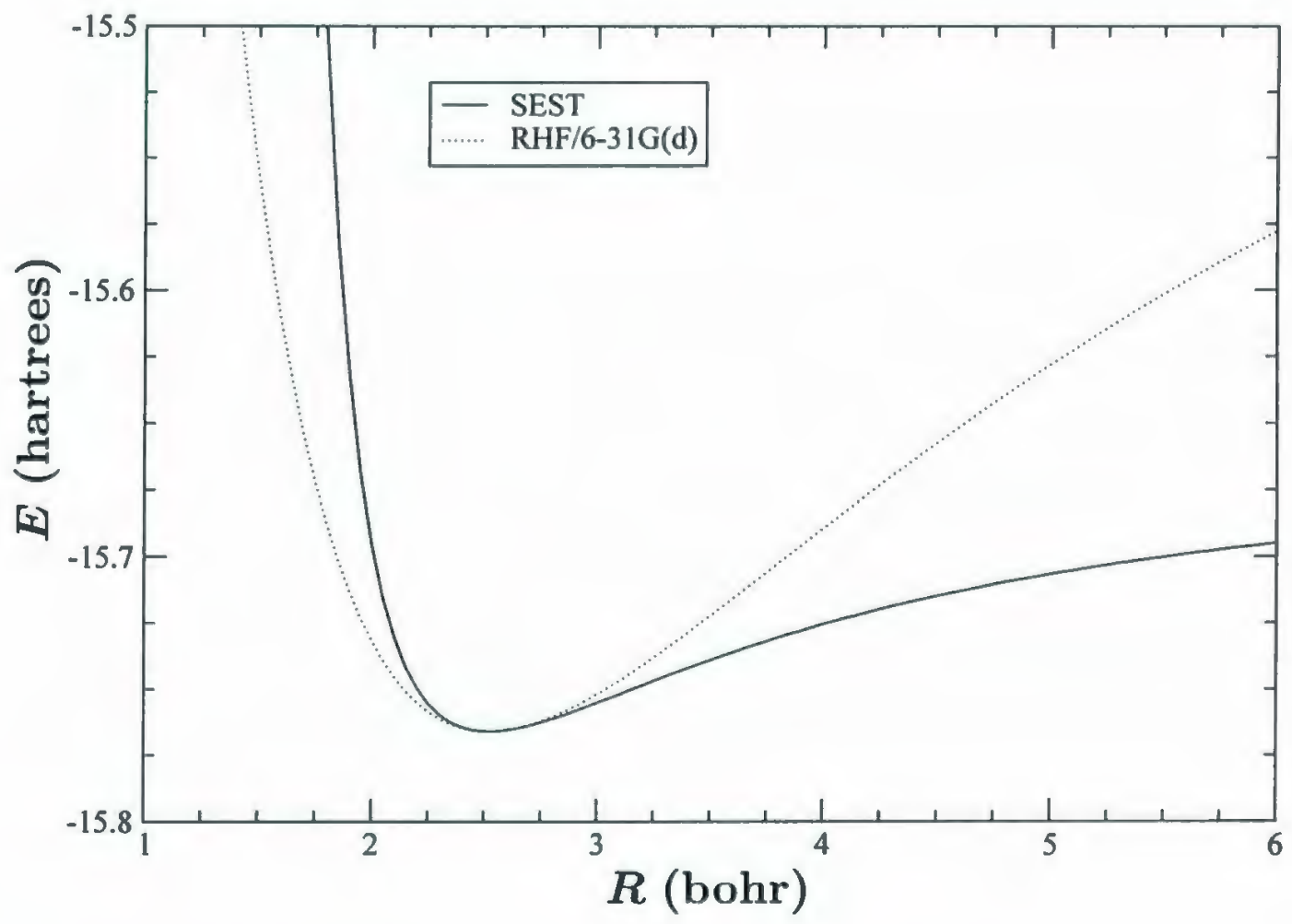

Figure 5.6. Dissociation curve for $\mathrm{BeH}$ bond of $\mathrm{BeH}_{2}$ (SEST and $\mathrm{RHF} / 6-31 \mathrm{G}$ (d)). 


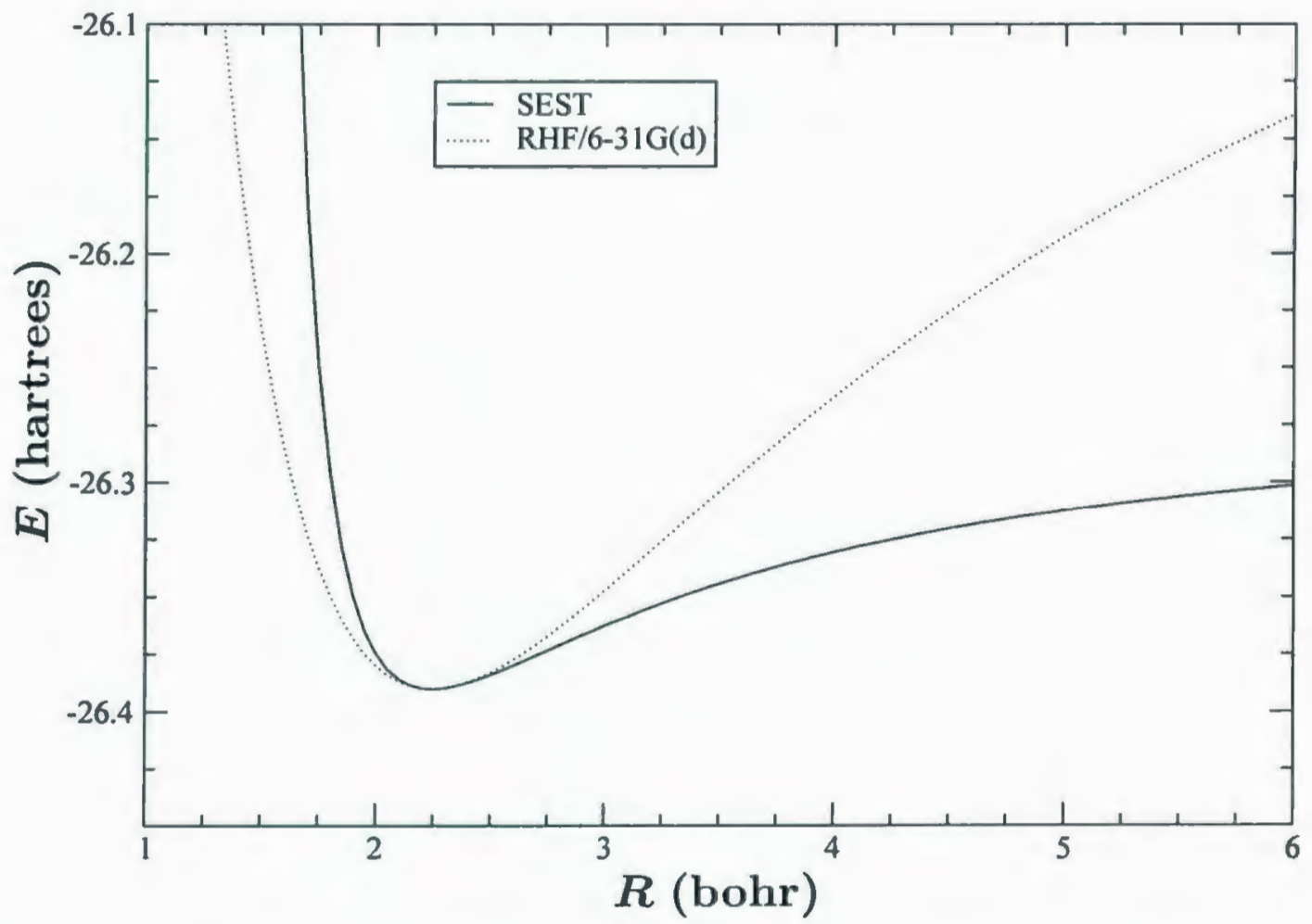

Figure 5.7. Dissociation curve for $\mathrm{BH}$ bond of $\mathrm{BH}_{3}$ (SEST and $\mathrm{RHF} / 6-31 \mathrm{G}(\mathrm{d})$ ). 


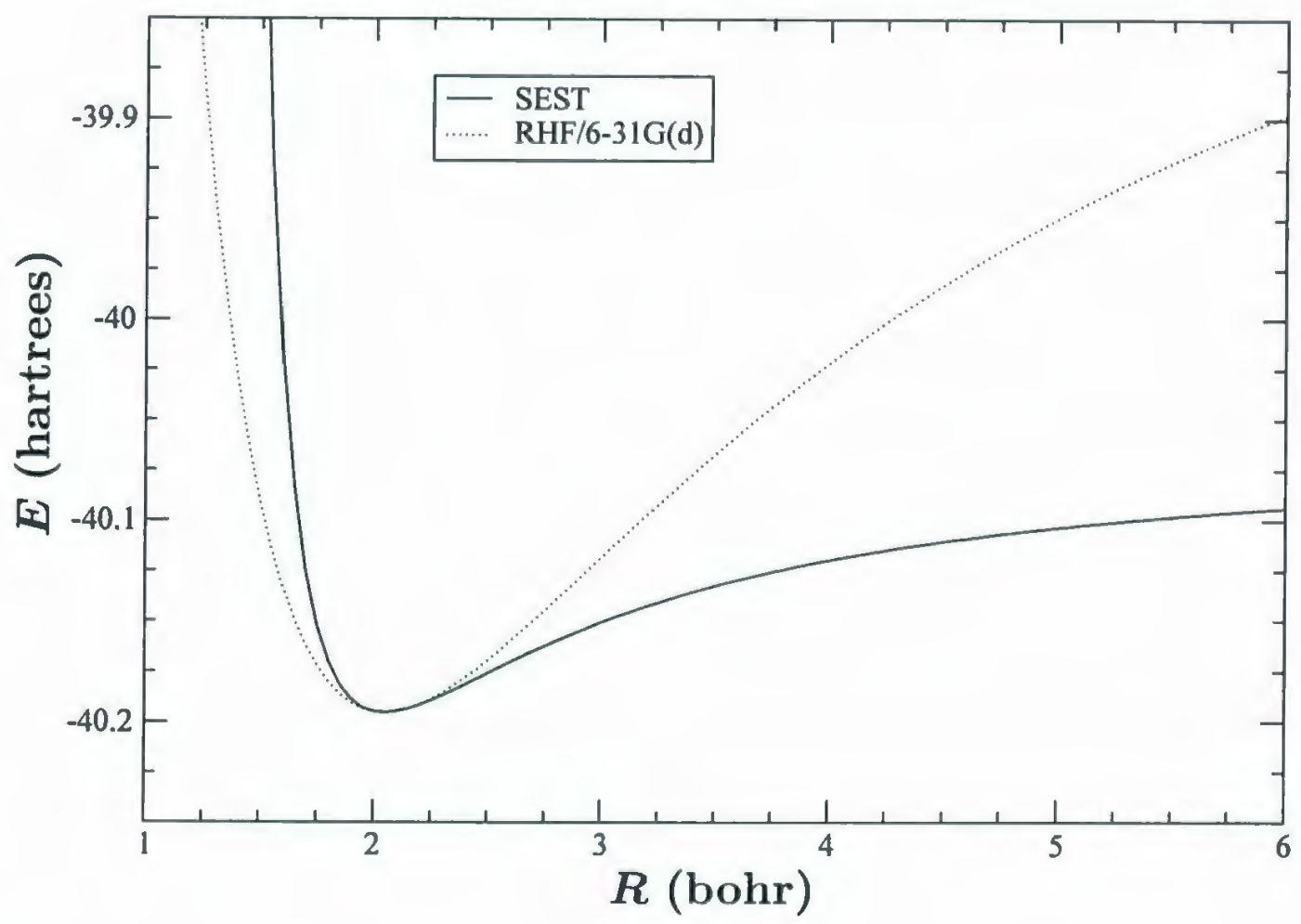

Figure 5.8. Dissociation curve for $\mathrm{CH}$ bond of $\mathrm{CH}_{4}$ (SEST and RHF/6-31G(d)). 


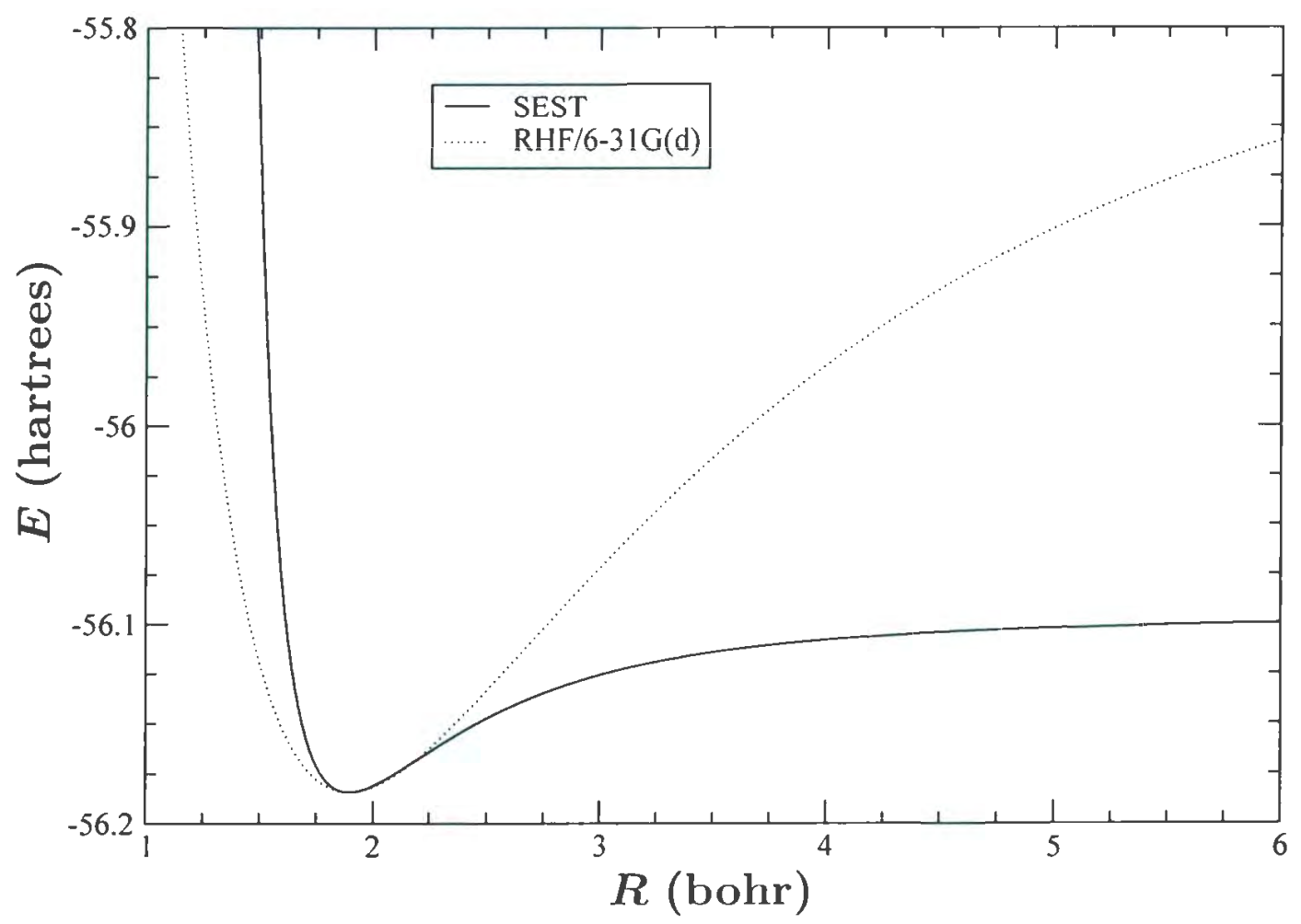

Figure 5.9. Dissociation curve for $\mathrm{NH}$ bond of $\mathrm{NH}_{3}$ (SEST and $\mathrm{RHF} / 6-31 \mathrm{G}(\mathrm{d})$ ). 


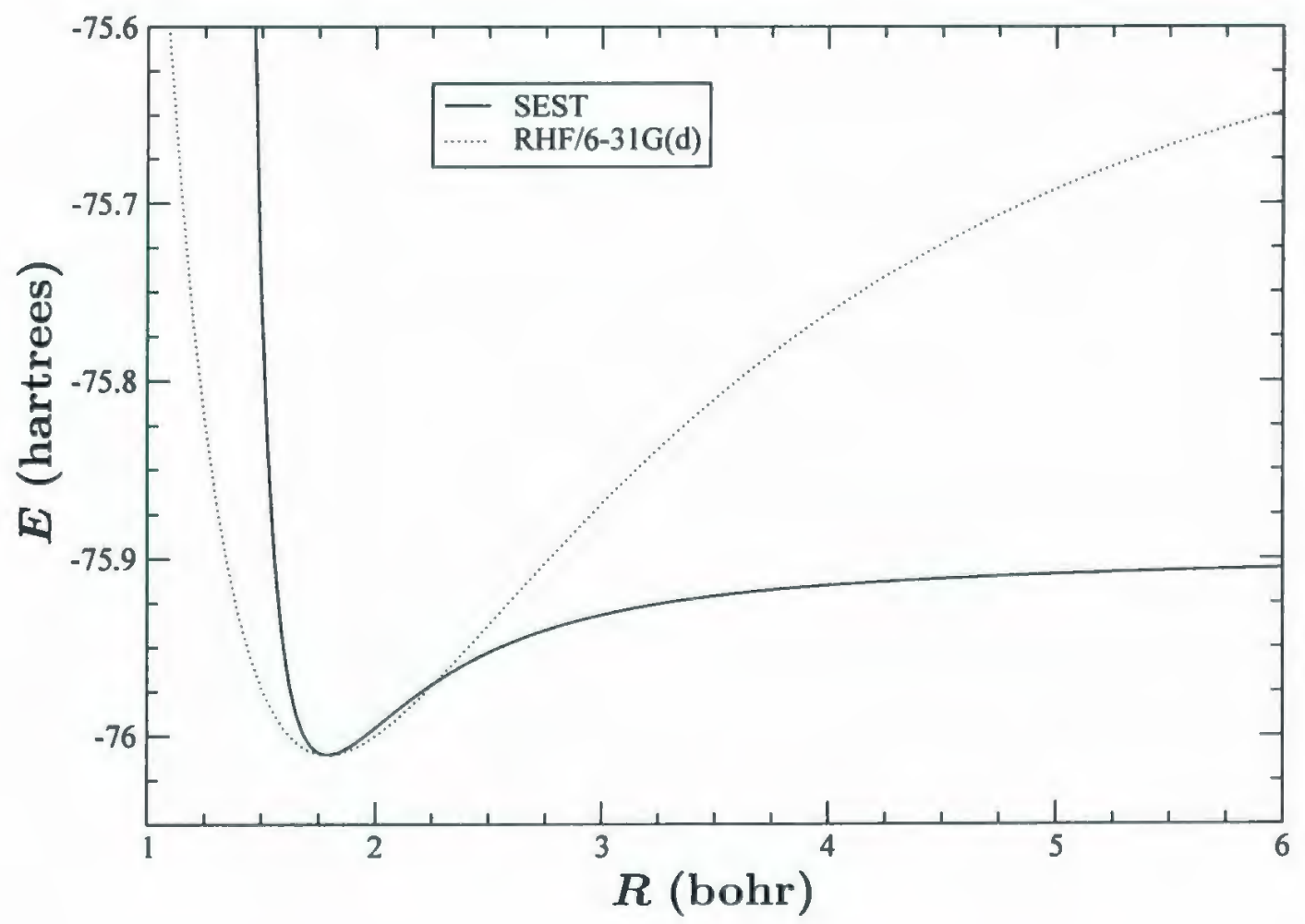

Figure 5.10. Dissociation curve for $\mathrm{OH}$ bond of $\mathrm{H}_{2} \mathrm{O}$ (SEST and RHF/6-31G(d)). 


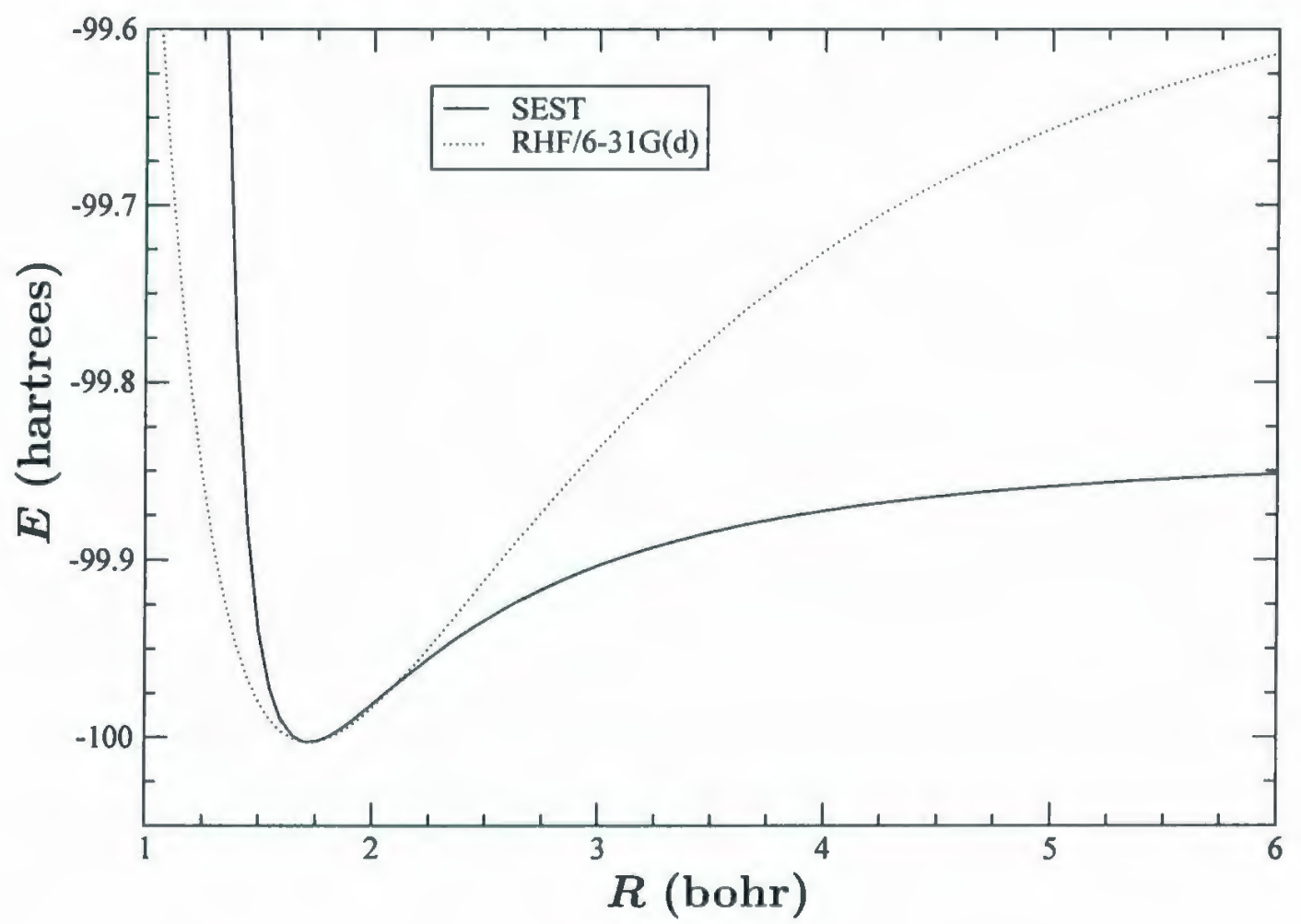

Figure 5.11. Dissociation curve for HF (SEST and RHF/6-31G(d)). 
exactly. Exact dissociation energies can be obtained with appropriate scaling. The ultimate goal of such a theory would be to accurately model several points along the bond dissociation curve as well as various points over the entire potential energy surface (i.e. conformational changes). An improved SEST model would use an $a b$ initio method such as GVB to obtain dissociation energy curve parameters, and first and second energy derivatives. However, the source for defining parameters is not limited to $a b$ initio calculations. A SEST approach could involve empirical parameters from experiment or a combination of $a b$ initio and empirical parameters.

Using the SEST formulation of this study, it is possible to fit some vibrational frequencies of the SEST models of the first-row hydrides to RHF/6-31G(d) values (Table 5.3).

For each hydride model, there is a hydrogen-hydrogen $(\mathrm{HH})$ non-bonding function. In an effort to explore the transferability of these functions, the same HH non-bonding function is used for all molecules. It was found that if the same $\mathrm{HH}$ non-bonding function is used, it is only possible to fit some of the vibrational frequencies.

For this study, the parameters of the $\mathrm{AH}$ bonding functions, where $\mathrm{A}$ is $\mathrm{Be}, \mathrm{B}$, $\mathrm{C}, \mathrm{N}, \mathrm{O}$, or $\mathrm{F}$, as well as the atom-lone pair, lone pair-lone pair interaction functions, were adjusted to fit the asymmetric stretch vibrational modes of the first-row hydrides. For $\mathrm{BeH}_{2}, \nu_{4}$ is the asymmetric stretch (Table 5.3). The error in the lower frequencies ranges from 133 to $223 \mathrm{~cm}^{-1}$. For the rest of the hydrides it is seen that the asymmetric stretches are fit exactly, however the symmetric stretch is always overestimated. The $\mathrm{BH}_{3}$ model overestimates the symmetric stretch, $\nu_{4}$, by $339 \mathrm{~cm}^{-1}$ and the error in the lower frequencies is less than $140 \mathrm{~cm}^{-1}$. For $\mathrm{CH}_{4}$ the symmetric stretch is overestimated by $695 \mathrm{~cm}^{-1}$, while the error in the other frequencies ranges 
TABLE 5.3: SEST and RHF/6-31G(d) vibrational frequencies for the first-row hydrides

\begin{tabular}{|c|c|c|c|}
\hline \multirow[b]{2}{*}{ System } & \multirow[b]{2}{*}{$\nu$} & \multicolumn{2}{|c|}{ Frequency $\left(\mathrm{cm}^{-1}\right)$} \\
\hline & & RHF /6-31G(d) & SEST \\
\hline \multirow[t]{4}{*}{$\mathrm{BeH}_{2}$} & 1 & 761 & 540 \\
\hline & 2 & 763 & 540 \\
\hline & 3 & 2107 & 2240 \\
\hline & 4 & 2324 & 2324 \\
\hline \multirow[t]{6}{*}{$\mathrm{BH}_{3}$} & 1 & 1225 & 1085 \\
\hline & 2 & 1305 & 1218 \\
\hline & 3 & 1306 & 1218 \\
\hline & 4 & 2694 & 3033 \\
\hline & 5 & 2816 & 2817 \\
\hline & 6 & 2818 & 2817 \\
\hline \multirow[t]{9}{*}{$\mathrm{CH}_{4}$} & 1 & 1487 & 1862 \\
\hline & 2 & 1488 & 1862 \\
\hline & 3 & 1488 & 1862 \\
\hline & 4 & 1702 & 1485 \\
\hline & 5 & 1702 & 1485 \\
\hline & 6 & 3196 & 3891 \\
\hline & 7 & 3303 & 3305 \\
\hline & 8 & 3305 & 3305 \\
\hline & 9 & 3307 & 3305 \\
\hline \multirow[t]{6}{*}{$\mathrm{NH}_{3}{ }^{a}$} & 1 & 1214 & 2294 \\
\hline & 2 & 1849 & 1980 \\
\hline & 3 & 1851 & 1980 \\
\hline & 4 & 3690 & 5001 \\
\hline & 5 & 3825 & 3825 \\
\hline & 6 & 3826 & 3825 \\
\hline \multirow[t]{3}{*}{$\mathrm{H}_{2} \mathrm{O}^{a}$} & 1 & 1829 & 2237 \\
\hline & 2 & 4070 & 5658 \\
\hline & 3 & 4191 & 4191 \\
\hline $\mathrm{HF}^{\mathrm{a}}$ & 1 & 4362 & 4362 \\
\hline
\end{tabular}

${ }^{a}$ Molecules with lone pairs have lone pair vibrational modes. These modes are not shown, they are of the order $10^{4}-10^{8} \mathrm{~cm}^{-1}$ and easily distinguished from the real modes. 
from $217 \mathrm{~cm}^{-1}$ to $375 \mathrm{~cm}^{-1}$. The overestimation of the symmetric stretch is greater for the molecules with lone pairs. The $\mathrm{NH}_{3}$ model has a symmetric stretch which is 1311 $\mathrm{cm}^{-1}$ larger than the RHF/6-31G(d) value. Also, the frequency of the out-of-plane bending mode, $\nu_{1}$, is overestimated by $1080 \mathrm{~cm}^{-1}$, while the error in the other two modes is only $130 \mathrm{~cm}^{-1}$. For $\mathrm{H}_{2} \mathrm{O}$, the symmetric stretch, $\nu_{2}$, and the bending mode, $\nu_{1}$, are also overestimated, by $1588 \mathrm{~cm}^{-1}$ and $408 \mathrm{~cm}^{-1}$ respectively. These overestimations are due to the $\mathrm{HH}$ non-bonding interaction. For the symmetric stretches, the out-of-plane bend of $\mathrm{NH}_{3}$, and the angle bend of $\mathrm{H}_{2} \mathrm{O}$, it is the $\mathrm{HH}$ distances which are changing the most. It appears that the $\mathrm{HH}$ non-bonding interaction is too strong for these vibrational modes. However, upon investigation of the SEST model of $\mathrm{CH}_{4}$, it was found that having a $\mathrm{HH}$ non-bonding function for a specific molecule does not necessarily correct the problem. By defining parameters specifically for the $\mathrm{HH}$ non-bonding interaction of $\mathrm{CH}_{4}$, vibrational modes $1,2,3$ and 6 to 9 (Table 5.3) were fit to the $\mathrm{RHF} / 6-31 \mathrm{G}$ (d) values to within $5 \mathrm{~cm}^{-1}$. However, vibrational modes 4 and 5 were underestimated by $942 \mathrm{~cm}^{-1}$. This suggests, that in order to fit all the vibrational frequencies of a molecule, modification of the functional form of at least the $\mathrm{HH}$ non-bonding interaction is required.

In the SEST model, lone pairs have their own coordinates, and therefore they also have vibrational frequencies. A mass of 0.0001 amu is assigned to the lone pairs in frequency calculations, which keeps the lone pair modes distinguishable and uncoupled from the real modes. With the exception of an out-of-plane bend, lone pair vibrational mode of $\mathrm{HF}, \nu_{l p}=5.7 \times 10^{4} \mathrm{~cm}^{-1}$, the lone pair vibrational modes are four to five orders of magnitude larger than the real modes. 


\subsection{Ethane}

It is essential that any modelling approach, which is intended for large systems, accurately describes carbon-carbon (CC) bonds. SEST can model the RHF/6-31G(d) equilibrium structure of ethane and the dissociation of the $\mathrm{CC}$ bond to $2 \mathrm{CH}_{3}, \mathrm{UHF} / 6$ 31G(d) (see Figure 5.12). The SEST model of ethane is constructed using newly

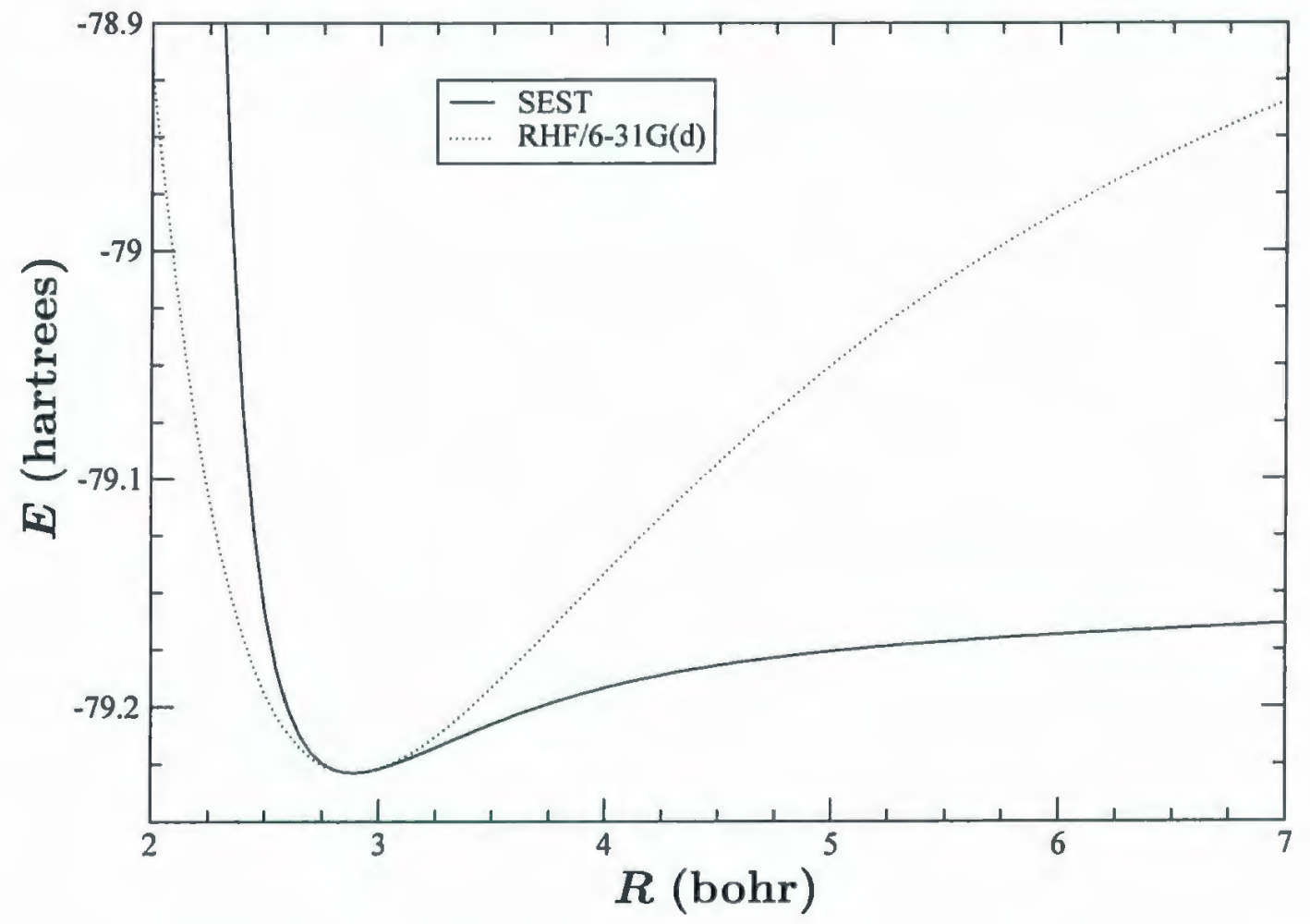

Figure 5.12. Dissociation curve for CC bond of ethane (SEST and RHF/6-31G(d).

defined $\mathrm{CC}$ bond and $\mathrm{CH}$ non-bonding functions along with the previously defined $\mathrm{HH}$ non-bonding function and a modified $\mathrm{CH}$ bond function from methane. The $\mathrm{CH}$ bond function of the SEST model of methane contains two non-zero parameters, $r_{\mathrm{C} i \mathrm{H} j}^{e e}=1.29131654 \mathrm{bohr}$ and $r_{\mathrm{HC} i}^{N e}=0.86020816 \mathrm{bohr}$. In order to fit the equilibrium 
geometry of ethane, the $\mathrm{CH}$ bond function is slightly modified; the value of a third parameter, $r_{\mathrm{CH} i}^{N e}$, is adjusted from 0 to $-0.04518330 \mathrm{bohr}$. Such an approach could be applied to the construction of a SEST force field. The parameters defined through modelling the first-row hydrides could be considered starting points for models of $\mathrm{CH}$ bonds, $\mathrm{OH}$ bonds, etc.. Especially in the case of hydrocarbons, parameters can be defined for small aliphatic systems and, as they are extended, the parameters are adjusted to some limiting, ideal value.

From the CC bond dissociation curve (Figure 5.12), it is seen that it is similar to that of the hydrides. Besides fitting the equilibrium geometry and qualitatively dissociating the CC bond correctly, the SEST model fits the rigid rotation about the $\mathrm{CC}$ bond in ethane. The same $\mathrm{HH}$ non-bonding function (same parameters) which is used for the first-row hydrides is used in the ethane model, which reproduces the potential energy curve for the rigid rotation about the CC bond at RHF/6-31G(d) exactly (Figure 5.13).

As a consequence, the vibrational mode which involves rotation about the CC bond, that has a value of $335 \mathrm{~cm}^{-1}$ at RHF/6-31G(d), has a value of $326 \mathrm{~cm}^{-1}$ for the SEST model. Also, through adjustment of CC bond parameters, the CC bond stretch vibrational mode, $1063 \mathrm{~cm}^{-1}$ at $\mathrm{RHF} / 6-31 \mathrm{G}(\mathrm{d})$, is fit exactly. The differences between SEST and RHF for the other vibrational frequencies range from $10 \mathrm{~cm}^{-1}$ to $1000 \mathrm{~cm}^{-1}$. The fitting of all such vibrational frequencies requires more investigation and experimentation with parameters and possibly functional forms. 


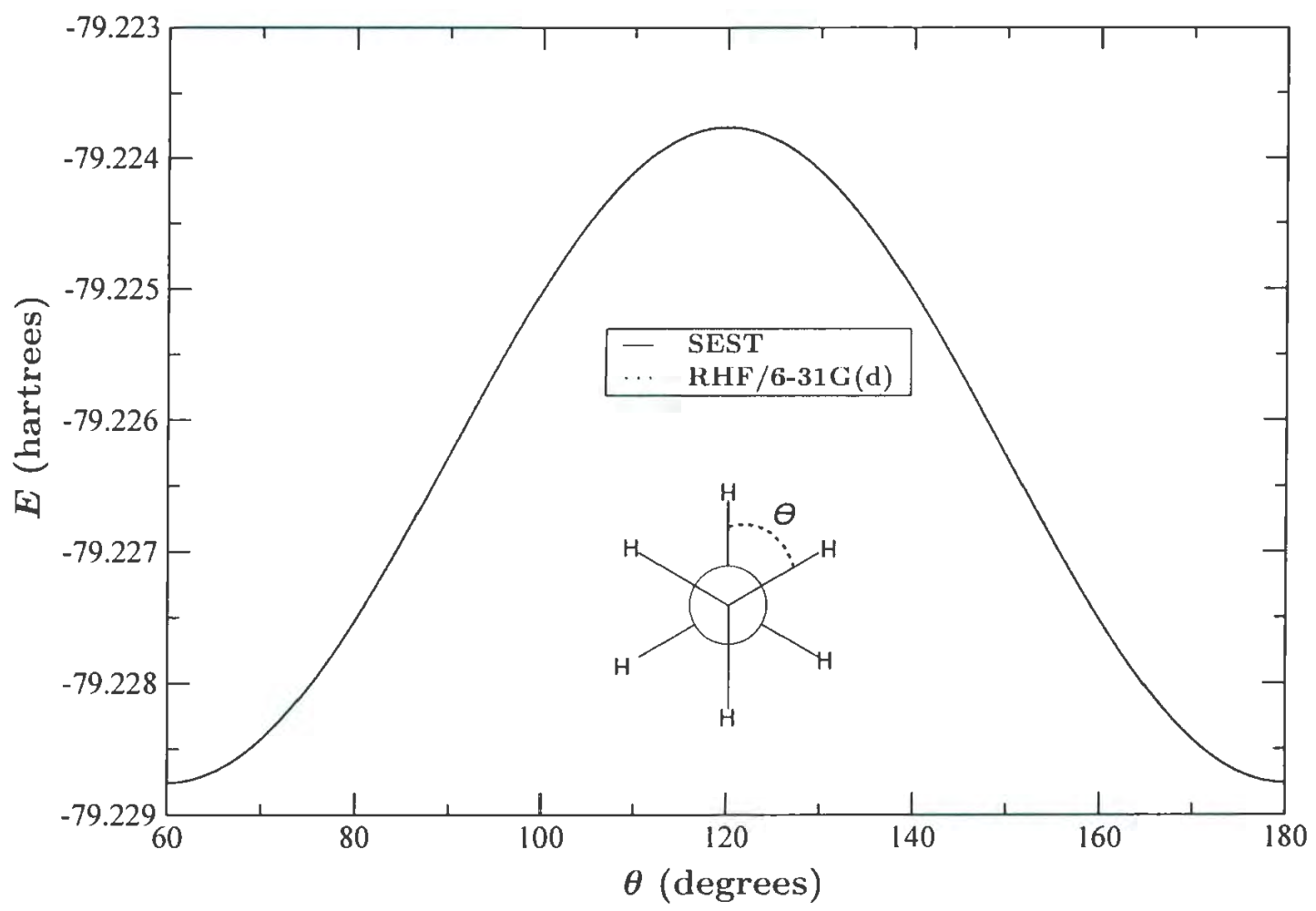

Figure 5.13. Rigid rotation about $\mathrm{CC}$ bond of ethane (SEST and RHF/6-31G(d)). 


\section{Conclusions}

It is indeed possible to implement a model of the electronic structure of a molecule that depends solely on atomic distances. It is shown that Simulated Electronic Structure Theory can accurately model MO energy components of equilibrium structures as well as the dissociation of bonds while providing an approximation of the MO energy components along the potential energy surface. In this study, the kinetic energy of the electrons is neglected to simplify the energy expression. However, the inclusion of the kinetic energy is quite feasible and would likely lead to similar results. Also, the model in this study fit the RHF/6-31G(d) equilibrium energies and geometries exactly and the UHF/6-31G(d) energy of the products of bond dissociation, multiplied by the factor $\frac{c_{v}-1}{c_{v}}$. The motivation for defining parameters from these levels of theory is due to availability and how quickly results can be obtained. More accurate SEST models could be obtained through use of higher levels of theory, GVB, Configuration Interaction, etc. It is also not necessary to define parameters from $a b$ initio calculations. Parameters may be empirically defined to fit experimental data. How parameters are defined will likely be determined by the intended use of the model.

It was seen that while this formulation of SEST fits the equilibrium structures, energies, and dissociation energies, it only fits some of the HF vibrational frequencies. The design of a SEST version that accurately predicts all of the vibrational frequencies of molecules may be possible. However, the SEST version presented in this study, the parameters and the functional form, will not predict all the vibrational frequencies of most molecules. It is suggested that the use of a modified functional form may solve this problem, especially in the case of non-bonding functions. An in-depth 
investigation of the dependence of the vibrational frequencies on functional form is required.

Further work is also required on the generation of a SEST force field. The advantage of a theory for which parameters are derived from $a b$ initio calculations is the ability to automate the generation of a force field. The wave functions of a test set of molecules could be used to determine the parameters of bonding and non-bonding functions according to a standard energy partitioning scheme and through fitting of the equilibrium structures and vibrational frequencies.

Finally, the most important aspect of the SEST approach is the explicit inclusion of the electrons. A molecular modelling theory which includes electronic structure has significant advantages over existing molecular mechanics methods. While this study showed that the relative positions of electrons and nuclei are related to atomic distances and consequently, the MO energy components, there are other properties related to the average interparticle distances. Similar to how the energy is related to the molecular structure, electronic properties such as dipole moment could also be modelled. Of course, as seen in this study, the inclusion of electrons allows for the breaking and forming of bonds and hence a theory that may be used to study kinetics. A simulated electronic structure theory creates many possibilities and much future work is required to explore them all.

\section{Acknowledgements}

The authors would like to thank the National Sciences and Engineering Research Council of Canada (NSERC) for funding and the Atlantic Computational Excellence Network (ACENet) for computational resources. 


\section{References}

(1) V. R. Coluci, S. O. Dantas, A. Jorio, and D. S. Galvão. Phys. Rev. B, 75:075417, 2007.

(2) F. E. Boas and P. B. Harbury. J. Mol. Biol., 380:415-424, 2008.

(3) J. P. Bowen and N. L. Allinger. Molecular mechanics: The art and science of parameterization. Reviews in Computational Chemistry, 2:81-97, 1991.

(4) A. Warshel and M. Levitt. J. Mol. Biol., 103:227-249, 1976.

(5) P. Amara and M. J. Field. Combined quantum mechanical and molecular mechanical potentials. In Encyclopedia of Computational Chemistry, pages 431436. Wiley, second edition, 1998.

(6) D. H. Andrews. Phys. Rev., 36:544-554, 1930.

(7) J. W. Hollett and R. A. Poirier. J. Theoretical Computational Chem., 6:13-22, 2007.

(8) J. W. Hollett and R. A. Poirier. J. Mol. Model., 15:739-745, 2009.

(9) S.F. Boys. Quantum Theory of Atoms, Molecules and the Solid State. Academic Press, 1966.

(10) I. R. Epstein. J. Chem. Phys., 53:4425-4436, 1970.

(11) D. R. Garmer and W. J. Stevens. J. Phys. Chem., 93:8263-8270, 1989.

(12) S. Liu, J. M. Pérez-Jordá, and W. Yang. J. Chem. Phys., 112:1634-1644, 2000. 
(13) W. Li and S. Li. J. Chem. Phys., 122:194109, 2005.

(14) A. Genoni and M. Sironi. Theo. Chem. Acc., 112:254-262, 2004.

(15) R. A. Poirier and J. W. Hollett. MUNgauss (Fortran 90 version). Chemistry Department, Memorial University of Newfoundland, St. John's, NL, A1B 3X7. With contributions from S. D. Bungay, A. El-Sherbiny, T. Gosse, D. Keefe, A. Kelly, C. C. Pye, D. Reid, K. Saputantri, M. Shaw, M.S. Staveley, Y. Wang and J. Xidos. 
The following Chapter, Chapter 6, is currently unpublished. Research therein was performed by J. W. Hollett under the supervision of R. A. Poirier. Segments of the literature review and research will be used for future proposals and included with subsequent research for publication. 


\section{Chapter 6}

\section{Development of a new electron correlation theory}

\section{Introduction}

\subsection{Electron correlation}

The electron correlation problem is one that persists throughout computational chemistry, and while it has several solutions, none are generally applicable to chemically interesting problems. The correlation energy, $E_{\text {corr }}$, is defined as the difference between the exact non-relativistic energy, $\mathcal{E}_{0}$ and the Hartree-Fock limit energy, E

$$
E_{\mathrm{corr}}=\mathcal{E}_{0}-E_{\mathrm{HFlim}}
$$

The Hartree-Fock limit refers to Hartree-Fock in the limit of an infinite, or complete, basis set. While the Hartree-Fock approximation generally provides approximately $99 \%$ of the total energy of atomic and molecular systems, the remaining $1 \%$ is found to be crucial in determining properties such as bond energies, equilibrium geometries, 
dipole moments, and many other properties of the molecular wave function. ${ }^{1}$ Consequently, properties that depend on molecular energies, such as reaction kinetics, also cannot be determined accurately without inclusion of the correlation between electrons.

The correlation problem is not unique to quantum chemistry. Correlation of the motion of more than two bodies is the many-body problem which is also found in classical mechanics, such as planets in the solar system. ${ }^{2}$ In quantum mechanics, the need to correlate the motion of the electrons arises from the Coulomb operator of the electronic Hamiltonian, $\widehat{H}$.

$$
\widehat{H}=-\sum_{i=1}^{N} \frac{1}{2} \nabla_{i}^{2}-\sum_{i=1}^{N} \sum_{A=1}^{M} \frac{Z_{A}}{r_{i A}}+\sum_{i=1}^{N} \sum_{j>i}^{N} \frac{1}{r_{i j}}
$$

where $N$ is the number of electrons and $M$ is the number of nuclei. The last term of the Hamiltonian (Equation 6.2) is the Coulomb operator which is the reciprocal of the interelectronic distance, $r_{i j}=\left|\mathbf{r}_{i}-\mathbf{r}_{j}\right|$. The electron correlation problem arises from the fact that the Hartree-Fock wave function, a Slater determinant, is constructed from one-electron functions. Solving the Hartree-Fock equations involves finding the optimum molecular orbitals in which each electron experiences the average effect of all the other electrons, known as a mean-field approximation. By this approximation, there is no accounting for the instantaneous interactions of the electrons, which is the missing electron correlation.

As a result of satisfying the Pauli antisymmetry principle, the Hartree-Fock wave function does account for Fermi correlation. Fermi correlation is due to the Fermionic character of the electrons and not the interaction of their charges. ${ }^{2}$ In the HartreeFock wave function, the motion of electrons with parallel spin is correlated through 
Fermi correlation and hence the probability of finding two electrons with parallel spin at the same point in space is zero. The Fermi correlation contribution to the HartreeFock energy is the exchange energy. The correlation that is missing from Hartree-Fock theory is the Coulomb correlation, which is the correlation of the motions of electrons due to the interaction of their charges. The mean-field approximation of HartreeFock does not describe these instantaneous interactions sufficiently and this missing correlation is often divided in terms of dynamical and non-dynamical correlation.

It is often useful to make the distinction between dynamical and non-dynamical electron correlation. Dynamical correlation involves the interactions between the electrons as they come close to one another, whereas non-dynamical correlation is a result of the inability of the single reference Hartree-Fock wave function to accurately describe the electronic configuration of a system due to near degeneracies. ${ }^{3}$ In such cases, the non-dynamical correlation energy is obtained by including the relevant reference configurations in a linear combination, which in general is referred to as Complete Active Space SCF (CASSCF). The non-dynamical correlation energy, $E_{\mathrm{ND}}$, can then be defined as,

$$
E_{\mathrm{ND}}=E_{\mathrm{CASSCFlim}}-E_{\mathrm{HFlim}}
$$

where both the CASSCF and HF energies are at the limit of a complete basis set. Dynamical and non-dynamical electron correlation are often discussed in terms of short range and long range effects. Non-dynamical correlation corresponds to the tendency of the electrons to be associated with certain nuclei, or regions of space, such as opposite atoms during bond dissociation and hence it is a long range effect. Whereas, dynamical correlation involves the inability of Hartree-Fock to describe the 
interaction of electrons as they come close together.

Other subdivisions and terminology for the electron correlation exist which may or may not prove useful in developing methods to deal with the correlation problem. Non-dynamical correlation is also referred to as left-right correlation $^{1}$ which implies the tendency of electrons to move to different nuclei as opposed to being on each nucleus an equal amount of time. Dynamical correlation can be subdivided into radial and angular correlation. Radial, or in-out, correlation corresponds to the requirement of additional electronic configurations that include atomic orbitals of the same angular quantum number but higher principal quantum number to more accurately describe the correlation between electrons, for example, using the $2 \mathrm{~s}$ and $3 \mathrm{~s}$ orbitals to describe $\mathrm{He}\left(1 \mathrm{~s}^{2}\right)$. Angular correlation is comprised of the correlation energy obtained through introducing configurations with higher angular quantum numbers. Such partitioning of the correlation energy is useful for analysis of electron correlation in different systems $^{3}$ which provides insight into the development of new approaches. A partitioning scheme designed for such a purpose has been given by Clementi, ${ }^{4}$ in which the correlation energy is divided into atomic, covalent, ionic, and van der Waals contributions.

\subsection{Explicitly correlated wave functions and exact energies of atomic sys- tems}

For small systems, it is possible to obtain correlated wave functions that are very near exact. This was first realized by Hylleraas, ${ }^{5}$ when he determined the helium wave function, assuming the form,

$$
\psi\left(\mathbf{r}_{1}, \mathbf{r}_{2}, \mathbf{r}_{12}\right)=e^{-\zeta\left(\mathbf{r}_{1}+\mathbf{r}_{2}\right)} e^{\gamma \mathbf{r}_{12}}
$$


which includes the interelectronic distance, $\mathbf{r}_{12}$, along with the positions of the two electrons with respect to the nucleus, $\mathbf{r}_{1}$ and $\mathbf{r}_{2}$. Hylleraas' calculations predicted the first ionization potential of helium to be $24.58 \mathrm{eV}$, which differs from the experimental value $(24.59 \mathrm{eV})$ by $0.01 \mathrm{eV} .^{2}$ Although highly accurate, Hylleraas' approach becomes significantly more complicated for systems with even three electrons and is therefore quite impractical. Despite this complexity, investigations still continue into explicitly correlated wave functions. ${ }^{6,7}$ Hylleraas is also responsible for expressing the exact energy, actually $E / Z^{2}$, of the helium-like ion as a Laurent series of the nuclear charge, $Z$, using perturbation theory. ${ }^{8}$ The expansion of the exact non-relativistic, $E(N, Z)$, Hartree-Fock, $E_{\mathrm{HF}}(N, Z)$, and correlation energies, $E_{\mathrm{corr}}(N, Z)$, were estimated by Davidson et al. for isoelectronic series of 2 to 18 electrons, $N$, using experimental and $a b$ initio values. ${ }^{9}, 10$

$$
\begin{gathered}
E(N, Z)=B_{0}(N) Z^{2}+B_{1} Z+B_{2}(N)+B_{3}(N) Z^{-1}+\ldots \\
E_{\mathrm{HF}}(N, Z)=B_{0}^{\mathrm{HF}}(N) Z^{2}+B_{1}^{\mathrm{HF}}(N) Z+B_{2}^{\mathrm{HF}}(N)+B_{3}^{\mathrm{HF}}(N) Z^{-1}+\ldots \\
E_{\text {corr }}(N, Z)=\Delta B_{0}(N) Z^{2}+\Delta B_{1}(N) Z+\Delta B_{2}(N)+\Delta B_{3}(N) Z^{-1}+\ldots
\end{gathered}
$$

Each $N$ dependent coefficient in the correlation energy expansion is given by

$$
\Delta B_{i}(N)=B_{i}(N)-B_{i}^{\mathrm{HF}}(N)
$$

The correlation energy as a function of $Z$, for $N=2-12$, is given in Figure 6.1.

With the exception of $N=4-7$ and 12, the first two coefficients of the exact and Hartree-Fock energy expansions are equivalent, ${ }^{10}$ and hence they do not appear in the correlation energy expansion. In these cases, there are small changes in $E_{\text {corr }}(N, Z)$ for the first low $Z$ values and then $E_{\text {corr }}(N, Z)$ approaches its limiting 


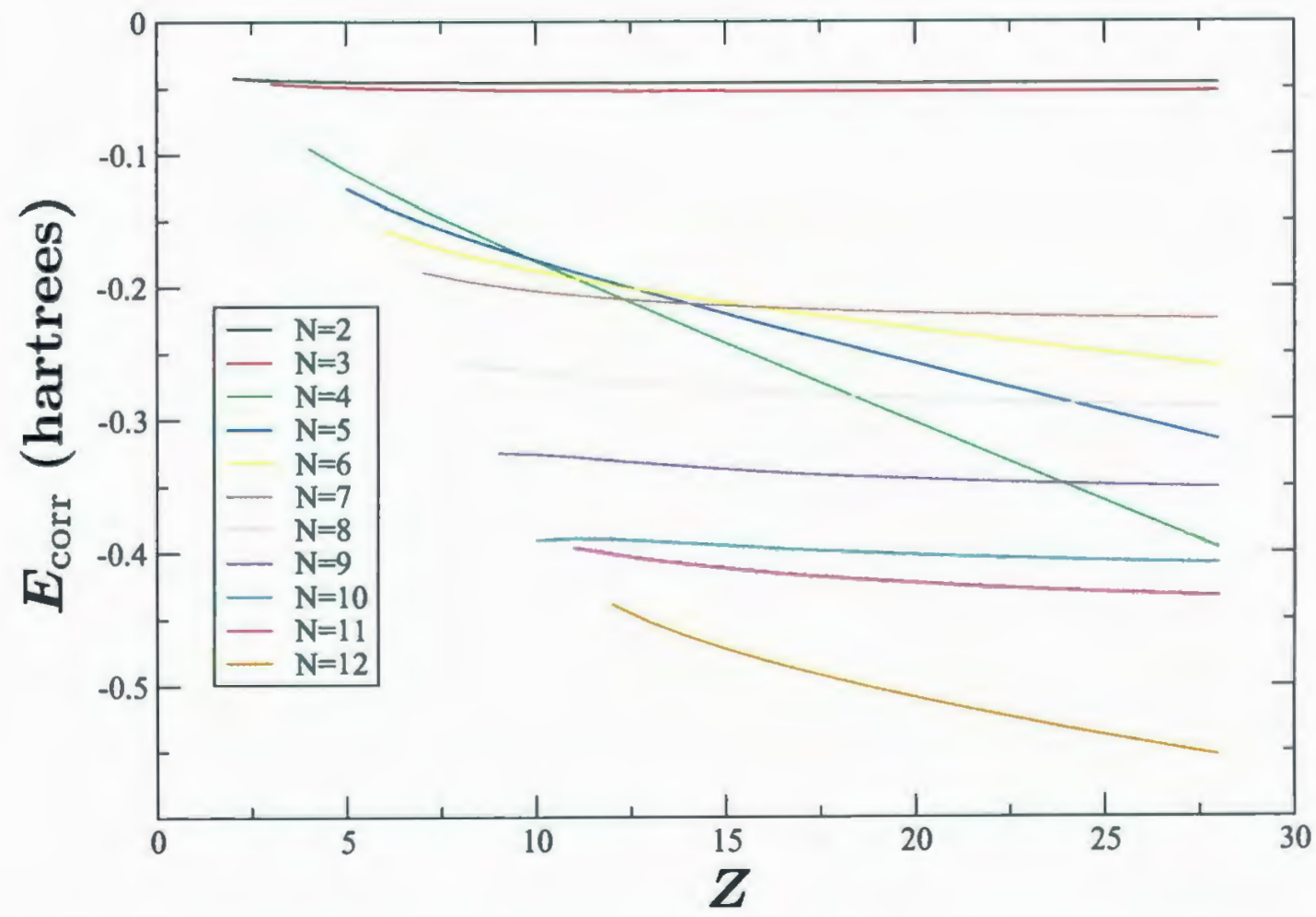

Figure 6.1. Correlation energy as a function of nuclear charge, $Z$, for isoelectronic series of 2 to 18 electrons $(N=2-12)$. 
value, $\Delta B_{2}(N)$. For the systems with four to seven and twelve electrons, the second coefficient of the expansion does not cancel. This is due to the inaccurate description of the system by a single reference Hartree-Fock wave function, or the aforementioned non-dynamical correlation energy. Unoccupied hydrogen-like $2 \mathrm{~s}$ and $2 \mathrm{p}$ orbitals are degenerate. In the case of four-electron isoelectronic series, the occupation of the orbitals lifts the degeneracy due to electron-electron repulsion, however the $1 s^{2} 2 s^{2}$ and $1 \mathrm{~s}^{2} 2 \mathrm{p}^{2}$ configurations remain nearly degenerate, and approach degeneracy as $Z \rightarrow \infty$.

The non-dynamical correlation present in these systems leads to a correlation energy expansion, $E_{\text {corr }}(N, Z)$, that is linear in $Z$.

Near exact correlated wave functions can only be determined for the simplest systems. Expansions of the correlation energy of atoms and ions in terms of nuclear charge provide only the energy and cannot be extended to molecules in a straightforward manner. However, both of these developments supply important data for approaching the correlation problem. Similar work has also been done concerning a hypothetical atom, referred to as the Hookium atom. ${ }^{11}$ The Hookium atom has the same Hamiltonian as helium-like ions except the nuclear attraction potential is replaced by a harmonic (Hooke's law) potential. The Hookium system provides an alternative system for which properties can be determined extremely accurately. For either system, the correlated wave functions provide a tool for measuring the effects of electron correlation, whereas the correlation energies provided by the Laurent expansions can be used to determine the quality of approximate, "correlated" methods. 


\subsection{Traditional wave function methods}

The correlation problem can be dealt with in a variety of ways, by several methods that are formally exact but have their own approximations and subsequent faults. Methods that involve expanding the wave function in terms of excited configurations of a reference wave function, which is normally the Hartree-Fock wave function, include configuration interaction (CI) and coupled-cluster theory (CC). The CI wave function is a linear expansion,

$$
\left|\Psi_{\mathrm{CI}}\right\rangle=\sum_{I} c_{I}\left|\Psi_{I}\right\rangle
$$

which, when substituted into the Schrödinger equation, leads to the matrix equation

$$
\mathrm{Hc}=\mathbf{E c}
$$

where $\mathbf{H}=\left\langle\Psi_{I}|\widehat{H}| \Psi_{J}\right\rangle$ is the CI matrix, $\mathbf{c}$ is the CI vector of coefficients, and $\mathbf{E}$ is a diagonal matrix of configuration energies. The above equation is solved by diagonalizing the $\mathrm{CI}$ matrix, $\mathrm{H}$. The dimension of the full CI problem grows factorially with the number of electrons and basis functions and therefore a full CI solution is not possible for most chemical systems. ${ }^{12}$ Therefore, the $\mathrm{CI}$ expansion is normally truncated at some $n$-tuple excitation, such as CI with single and double excitations (CISD). The size of the CI vector, $c$, is often so enormous that it cannot be stored in memory for many calculations. This led to the development of direct $\mathrm{CI}$ methods which use Slater determinants as opposed to spin eigenfunctions (i.e. configuration state functions (CSFs)) to efficiently evaluate the required coupling coefficients. ${ }^{13}, 14$ Algorithms solving the CI problem are also accelerated by exploiting the significant sparsity of the $\mathrm{CI}$ matrix, $\mathrm{H}$, as a large number of electronic configurations do not 
contribute at all to the CI wave function. ${ }^{15}$ Full CI methods are quite useful in providing a benchmark for other correlated methods, as they are extremely reliable due to their variational nature. Unfortunately, the advances in computational power and algorithm development have not made full CI methods applicable to general chemical problems and will not in the near future. Therefore, it is necessary to truncate the CI expansion which has a well known drawback.

Truncated CI methods are not size consistent, meaning the energy of infinitely separated systems are not additive. This problem was circumvented by the development of quadratic CI (QCI) methods, ${ }^{16}$ which in the spirit of coupled-cluster (CC) theory, include products of excitations, such as disconnected triples, $\hat{T}_{1} \hat{T}_{2}$, where $\hat{T}_{n}$ is the $n$-tuple excitation operator. The minimal number of terms from the CC expansion, necessary for size consistency, are included. ${ }^{17}$ The cluster expansion of the reference wave function, $\left|\Psi_{0}\right\rangle$, is given by

$$
\left|\Psi_{\mathrm{CC}}\right\rangle=e^{\hat{T}}\left|\Psi_{0}\right\rangle
$$

where $\hat{T}=\sum_{n=1}^{N} \hat{T}_{n}$ and $N$ is the number of electrons. In CC theory, the expansion is normally truncated at the double excitation operator. However, higher order excitations, such as triple and quadruple excitations, are approximated by their disconnected analogues. The CC approximation is size consistent at any level of truncation which is why it has become a standard for the calculation of highly accurate wave functions, especially coupled-cluster singles and doubles including a non-iterative approximation of the triple excitations, $\operatorname{CCSD}(\mathrm{T}){ }^{1}$

As an alternative to CI and CC methods, Møller-Plesset perturbation theory provides a size consistent approach to the correlation problem. A correlated wave 
function is not obtained, but corrections to the energy are obtained by applying a perturbation to the Hartree-Fock Hamiltonian. ${ }^{18}$ Contributions to the correlation energy are given by the second order and higher energy corrections, which correspond to the MP $n$ levels of theory. Similar to CI, the energy contributions can be divided in terms of contributions from single, double, etc., excitations. ${ }^{1}$ Kutzelnigg and Klopper $^{19}$ were able to improve convergence of MP2 calculations with respect to basis set by introducing $r_{12}$ dependent terms in a partial wave expansion, MP2-R12. The same approach has since been applied to $\mathrm{CI}^{20}$ and $\mathrm{CC}^{21}$ theories.

Given a single reference wave function, the truncated versions of $\mathrm{CI}$ and $\mathrm{CC}$ theory, as well as $\mathrm{MP} n$ theory, will recover a large percentage of the dynamical correlation energy. If the single reference wave function is a poor description of the system, such as a partially dissociated bond, only Full CI will account for the non-dynamical correlation. In such cases, multi-reference (MR), or multi-configurational (MC), wave functions are used, ${ }^{22}$ which are linear combinations of determinantal wave functions deemed necessary to describe the reference state.

$$
\left|\Psi_{\mathrm{MCSCF}}\right\rangle=\sum_{I} c_{I}\left|\Psi_{I}\right\rangle
$$

The MC wave function is determined by an SCF procedure (MCSCF), in which the energy is minimized with respect to both the expansion coefficients and the orbitals.

$$
E_{\mathrm{MCSCF}}=\min _{\left\{c_{r}, \phi_{i}\right\}}\left\langle\Psi_{\mathrm{MCSCF}}|\widehat{H}| \Psi_{\mathrm{MCSCF}}\right\rangle
$$

Different minimization schemes can be applied. In many cases, a Newton-Raphson or modified Newton-Raphson method is applied. ${ }^{23}$ The arbitrary selection of important electronic configurations for a $\mathrm{MC}$ wave function introduces a bias which can make 
MCSCF methods unreliable. However, specifying an active space of orbitals and using all possible configurations involving those orbitals, referred to as the complete active space (CASSCF), is a more general and reliable approach. The MCSCF wave function will account for the non-dynamical correlation energy, and hence provide a qualitatively correct description of the chemical system. ${ }^{1}$ For a quantitatively correct description, it is necessary to include the dynamical correlation energy by applying CI (MRCI), CC (MRCC), or MP2 (CASPT2) theories to the MR, or MC, wave function.

\subsection{Density Functional Theory}

The electron correlation problem can also be approached without a wave function at all, at least in theory. Density Functional Theory (DFT) defines the energy of an electronic system as a functional of the electron density.

$$
E[\rho(\mathbf{r})]=V_{\text {ext }}[\rho(\mathbf{r})]+F[\rho(\mathbf{r})]
$$

where $V_{\text {ext }}$ is the external potential and $F[\rho(\mathbf{r})]$ is the universal functional. For molecules in the absence of an external field, the external potential is equivalent to the nuclear-electron potential, $V_{\mathrm{ext}}[\rho(\mathbf{r})]=V_{\mathrm{Ne}}[\rho(\mathbf{r})]$. The universal functional is independent of the system and includes the kinetic energy of the electrons, $T[\rho(\mathbf{r})]$, and electron-electron potential energy, $V_{\text {ee }}[\rho(\mathbf{r})]$.

$$
F[\rho(\mathbf{r})]=T[\rho(\mathbf{r})]+V_{\mathrm{ee}}[\rho(\mathbf{r})]
$$

The electron-electron potential energy functional can be divided into the Coulomb repulsion and exchange-correlation, $E_{x c}[\rho(\mathbf{r})]$, functionals.

$$
V_{\mathrm{ee}}[\rho(\mathbf{r})]=\frac{1}{2} \iint \frac{\rho\left(\mathbf{r}_{1}\right) \rho\left(\mathbf{r}_{2}\right)}{r_{12}} d \mathbf{r}_{1} d \mathbf{r}_{2}+E_{x c}[\rho(\mathbf{r})]
$$


The Coulomb repulsion energy is the only component of the universal functional for which the expression in terms of density is known. However, the Coulomb repulsion functional does contain self-interaction error (SIE), which is evident in the fact that for a one-electron system the Coulomb repulsion energy is not zero. In Hartree-Fock theory, there is no SIE due to cancellation with the corresponding exchange integral.

The Kohn-Sham method was developed to deal with the inability to express the kinetic energy in terms of density. The Kohn-Sham approach involves defining a fictitious system of non-interacting electrons, which have the same density as the exact ground state density of the system, $\rho^{\mathrm{KS}}(\mathbf{r})=\rho_{0}(\mathbf{r})$. The kinetic energy of the non-interacting system, $T^{\mathrm{KS}}$, can then be expressed in terms of the Kohn-Sham orbitals, $\left\{\chi_{i}^{\mathrm{KS}}\right\}$.

$$
T^{\mathrm{KS}}=-\frac{1}{2} \sum_{i=1}^{N}\left\langle\chi_{i}^{\mathrm{KS}}(1)\left|\nabla_{1}^{2}\right| \chi_{i}^{\mathrm{KS}}(1)\right\rangle
$$

The Kohn-Sham exchange-correlation functional then also accounts for the correction to the kinetic energy of the system of non-interacting electrons, $\Delta T[\rho(\mathbf{r})]$. Minimizing the Kohn-Sham energy expression in terms of the Kohn-Sham orbitals, under the constraint that the orbitals remain orthonormal, leads to the Kohn-Sham equations.

$$
\left[-\frac{1}{2} \nabla_{1}^{2}-\sum_{A=1}^{M} \frac{Z_{A}}{r_{1 A}}+\int \frac{\rho\left(\mathbf{r}_{2}\right)}{r_{12}} d \mathbf{r}_{2}+\hat{v}_{x c}\left(\mathbf{r}_{1}\right)\right] \chi_{i}^{\mathrm{KS}}\left(\mathrm{r}_{1}\right)=\epsilon_{i} \chi_{i}^{\mathrm{KS}}\left(\mathrm{r}_{1}\right)
$$

The exchange-correlation operator, $\hat{v}_{x c}(\mathbf{r})$, is defined as the functional derivative of the exchange-correlation energy with respect to the density.

$$
\hat{v}_{x c}(\mathbf{r})=\frac{\delta E_{x c}[\rho(\mathbf{r})]}{\delta \rho(\mathbf{r})}
$$

The exact form of the exchange-correlation operator remains unknown, however several approximations exist. Generally, the exchange-correlation operator is ex- 
pressed as a sum of exchange, $\hat{v}_{x}(\mathbf{r})$, and correlation, $\hat{v}_{c}(\mathbf{r})$, parts.

$$
\hat{v}_{x c}(\mathbf{r})=\hat{v}_{x}(\mathbf{r})+\hat{v}_{c}(\mathbf{r})
$$

Such an expression is reasonable because one would expect that the density functional form of the exchange, or Fermi correlation, energy is separable from the energy due to kinetic energy correction, SIE correction, and electron correlation. One of the earliest forms of the exchange operator was derived by Bloch and Dirac, which is commonly referred to as the Slater exchange. ${ }^{24}$

$$
\hat{v}_{x}^{\text {Slater }}(\mathbf{r})=-\frac{3}{4}\left(\frac{3 \rho(\mathbf{r})}{\pi}\right)^{1 / 3}
$$

Exchange and correlation operators that are only dependent on the density to some power are classified as local density approximations (LDA), $\hat{v}_{x c}^{\mathrm{LDA}}[\rho(\mathbf{r})]$. More flexibility in the functional form is found if it is applied to the unrestricted system. The exchange-correlation operator then incorporates the spin-density, $\rho_{\alpha}(\mathbf{r})$ and $\rho_{\beta}(\mathbf{r})$, rather than just the total density, $\rho(\mathbf{r})=\rho_{\alpha}(\mathbf{r})+\rho_{\beta}(\mathbf{r})$, which is referred to as the local spin-density approximation (LSDA) (see Appendix A). However, the derivation of such formulations from the homogeneous electron gas leads to expected inaccuracy due to the inhomogeneous electron density of molecules. To address such a problem, the generalized gradient approximation (GGA) was introduced, in which the exchange and correlation operators incorporate the gradients of the spin densities, $\hat{v}_{x c}^{\mathrm{GGA}}\left[\rho_{\alpha}(\mathbf{r}), \rho_{\beta}(\mathbf{r}), \nabla \rho_{\alpha}(\mathbf{r}), \nabla \rho_{\beta}(\mathbf{r})\right]$. The exchange functional introduced by Becke ${ }^{25}$ referred to as $\mathrm{B} 88$ (or simply B) consists of a LSDA term and a complex integral over spin density and density gradients and hence is a GGA functional (Appendix A). The meta-GGA functionals incorporate the Laplacian, or kinetic energy density, into the 
exchange and correlation functions. The adaptation of the Colle-Salvetti correlation energy function to a density functional by Lee, Yang and Parr, ${ }^{26}$ the LYP functional (Appendix A), is a meta-GGA functional. It is the combination of these different levels of exchange and correlation operators that lead to the hybrid functionals that have been popular for the past decade. The Becke three-parameter exchange-correlation functional, B3LYP, contains a sum of the LSDA and B88 exchange functionals and the Hartree-Fock exchange, along with the VWN (LSDA) and LYP correlation energy functionals ${ }^{24}$ (Appendix A). The contribution of each functional is weighted by three empirical parameters fit to a data set. For over a decade, B3LYP has been a mainstay in DFT studies of chemical systems due to the computational speed of the Kohn-Sham method and the accuracy of the B3LYP functional. Becke has recently developed an updated exchange-correlation functional, DF07, ${ }^{27}$ which consists of exact Hartree-Fock exchange, dynamical and non-dynamical correlation functionals, and a functional for dispersion (Appendix A). Also recently, Truhlar and coworkers ${ }^{28}$ have introduced the M06 family of hybrid meta-exchange-correlation functionals which are best suited to organometallic, inorganic, and noncovalent interactions. New functionals which have been parametrized or fitted to larger data sets focusing on both covalent and noncovlaent interactions should certainly achieve higher accuracy in the determination of thermochemistry and kinetics. It is evident that the search for better approximations to the exact exchange-correlation functional continues.

Although DFT and the associated approximate exchange-correlation functionals have become the predominant method for general chemical calculations, the functionals are not without their flaws. There are fundamental problems associated with many density functionals such as behaviour at the high density limit, SIE, and size consis- 
tency. ${ }^{29}$ The failure of the exchange-correlation functionals at the high density limit, as it pertains to atoms and molecules, concerns the approximation of exchange and correlation energy as the nuclear charge becomes very large (i.e. $Z \rightarrow \infty$ ). This failure is illustrated by calculations on the simple isoelectronic series of helium-like ions (Figure 6.2). Given that the correlation energy accounted for by post Hartree-Fock methods is not equivalent to that of DFT, and the fact that the exchange contributions are not equivalent either, the total errors from the exact non-relativistic energy are compared here, $E_{\text {error }}=E_{\text {calc }}-E_{\text {exact }}$.

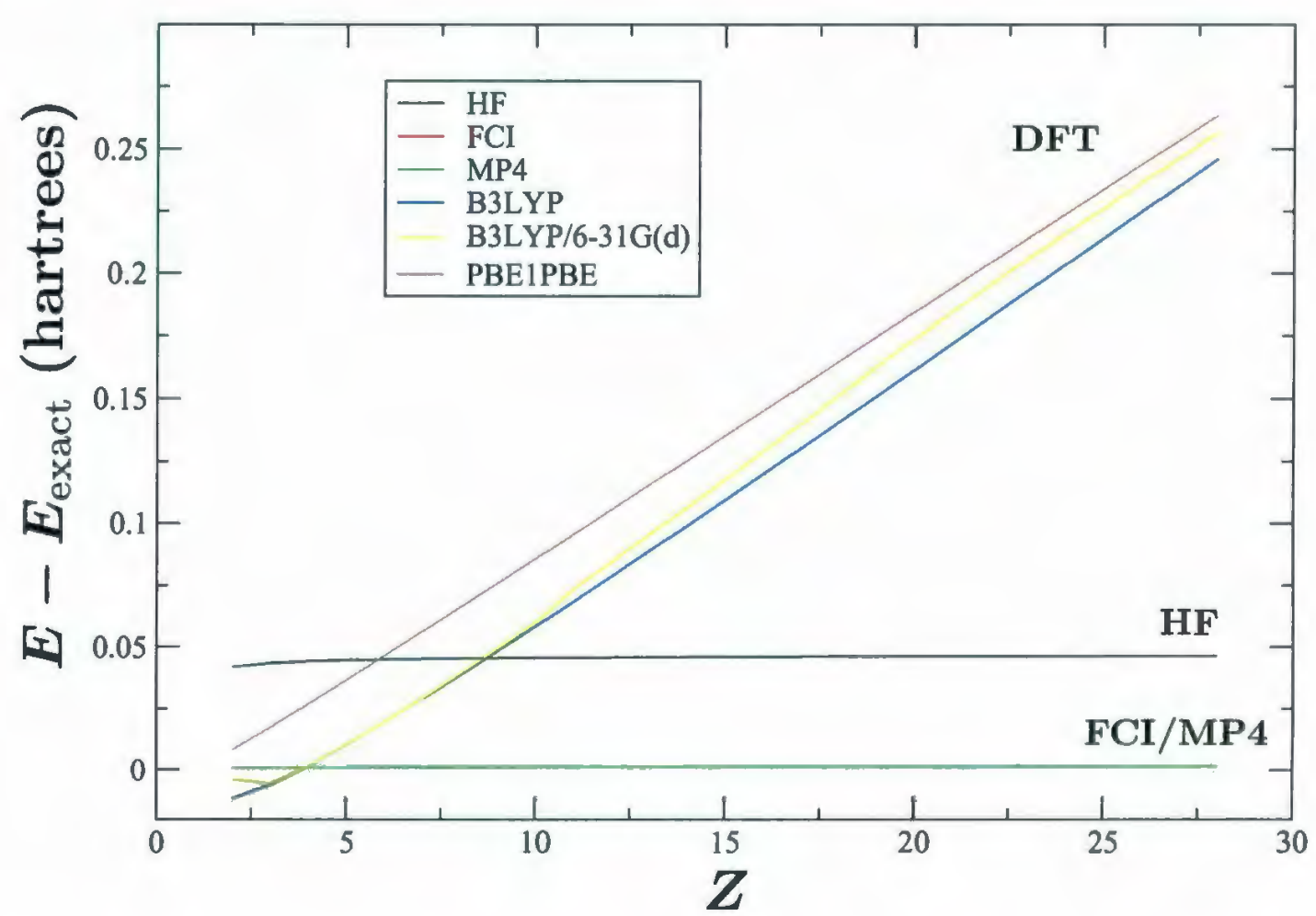

Figure 6.2. Error in energies of two electron helium-like ions, $Z=2-28$. (Other than B3LYP/6$31 \mathrm{G}$ (d) data, all calculations performed with a scaled aug-cc-pVQZ helium basis set.)

For two-electron systems, it is seen that exchange-correlation functionals PBE1PBE 
and the popular B3LYP deviate drastically from the exact energy and do not follow the behaviour of the post Hartree-Fock methods (MP2 and full CI). The post HartreeFock methods consistently account for approximately $98 \%$ of the correlation energy. As $Z$ increases, the DFT functionals are seen to rise above the exact energy and then above the Hartree-Fock energy. This failure is partially due to the fact that the functionals include an exchange contribution, which should be zero (no Fermi correlation) for the closed-shell, two-electron case. However, it is noted by Davidson et al. in a much more in depth study of this phenomenon ${ }^{30}$ that most correlation functionals, with the exception of LYP, diverge at large $Z$. In their study, they also note that no correlation functional effectively models the constant and linear behaviour of the correlation energy as a function of $Z$, which is observed for two-electron and four-electron systems, respectively (Figure 6.1). They found the best performance for a non-empirical meta-GGA correlation functional by Tao, Perdew, Staroverov, and Scuseria (TPSS).

\subsection{New approaches}

The behaviour of the exchange-correlation functionals, B3LYP and PBE1PBE (Figure 6.2), suggests that a more accurate approach to such fundamental problems is achieved by replacing the exchange functional by Hartree-Fock exchange, an exact analytical expression. It then remains to develop a functional to account for the correlation energy. This exact-exchange DFT approach has led to the development of several new correlation functionals, including Becke's DF07. Thakkar and coworkers have recently revived the Wigner correlation energy functional in a reparametrization where the parameters depend upon expectation values of the Hartree-Fock wave 
function. ${ }^{31}$ It appears as though a new generation of correlation functionals may emerge that can no longer benefit from a cancellation of error with the exchange functional. In the exact exchange formalism, SIE is corrected by the inclusion of Hartree-Fock exchange. However, some research has been devoted to treating this fundamental problem of exchange-correlation functionals. One particular approach minimizes SIE using Coulomb-attenuated functionals, which involves multiplying the Coulomb operator by the error function. ${ }^{32}$

Although DFT development has shifted significantly from exchange-correlation functionals to the exact-exchange and correlation functional formalism, many still believe density functional approaches are not the answer to the correlation problem. Gill, ${ }^{33}$ Giorgi, ${ }^{34-36}$ and others ${ }^{37}$ have begun the development of intracule functional theory (IFT). The basic premise of IFT is that the correlation energy can be expressed in terms of intracule functionals where the intracules are functions of, but not limited to, the relative positions, $r_{12}=\left|\mathbf{r}_{1}-\mathbf{r}_{2}\right|$ and momentum $p_{12}=\left|\mathbf{p}_{1}-\mathbf{p}_{2}\right|$ of two electrons. An analogous approach was suggested by Rassolov when he derived an expression for a Hartree-Fock electron correlation operator from the correlation energy of two-electron ions in the high density limit and the dense homogeneous electron gas. ${ }^{38}$ The extended Overhauser version of IFT developed by Giorgi and coworkers has been successful in describing the correlation energy of two-electrons in the high density limit. ${ }^{35}$ The approach was also extended to describing bond dissociation through separation of long and short range correlation effects, ${ }^{36}$ a scheme which has been previously applied to conventional density functionals. Gill has suggested several forms of intracule functionals, ${ }^{33}$ among them is the Wigner intracule which was subsequently tested on a set of light atom diatomics. ${ }^{37}$ Investigations continue 
into various intracule forms and their applicability. ${ }^{39}$

An approach that resembles IFT and DFT but differs in the basic construction of the correlation functional, is orbital functional theory. The idea was proposed by Nesbet in an article that proves a local kinetic or exchange energy operator does not exist for any more than two electrons. ${ }^{40}$ It is therefore likely that a local correlation operator also does not exist. An orbital functional theory involving fractionally occupied orbitals, as suggested by Nesbet, can account for kinetic and exchange energy exactly and allows for the construction of a non-local correlation operator. Nesbet also derives a formally exact but implicit expression for the correlation energy of orbital functional theory. He later suggests how unrestricted Hartree-Fock (UHF) could be extended to an orbital functional theory. ${ }^{41}$ The orbital functional theory Nesbet suggests is equivalent to natural orbital functional theory (NOFT) introduced by Goedecker and Umrigar. ${ }^{42}$ Goedecker and Umrigar argue, that by expressing the energy as a functional of natural orbitals and occupation numbers, the only unknown functional is the correlation energy. However, investigations by Scuseria and Staroverov $^{43}$ and Cohen and Baerends ${ }^{44}$ reveal shortcomings in the early natural orbital functionals, referred to as the corrected Hartree $(\mathrm{CH})$ and corrected HartreeFock (CHF) functionals. The investigation by Scuseria and Staroverov also showed promise for the Goedecker and Umrigar functional despite some fundamental flaws regarding $N$-representability. Scuseria and Staroverov also refer to natural orbital functional theory as density matrix functional theory (DMFT) ${ }^{43}$ or, to be more specific, first-order reduced density matrix functional theory (1-RDMFT) as the natural orbitals and their occupation numbers are the eigenfunctions and eigenvalues of the 
1-RDM. The 1-RDM, $\gamma\left(\mathbf{x}_{1}, \mathbf{x}_{1}^{\prime}\right)$, is defined by the integral

$$
\gamma\left(\mathbf{x}_{1}, \mathbf{x}_{1}^{\prime}\right)=N \int \ldots \int \Psi\left(\mathbf{x}_{1}, \mathbf{x}_{2}, \ldots, \mathbf{x}_{N}\right) \Psi\left(\mathbf{x}_{1}^{\prime}, \mathbf{x}_{2}, \ldots, \mathbf{x}_{N}\right) d \mathbf{x}_{2} \ldots d \mathbf{x}_{N}
$$

where $\Psi$ is the $N$ electron wave function and $\left\{\mathbf{x}_{i}\right\}$ are the spatial and spin coordinates of the electrons. Following the early attempts to develop NOFs, or DMFs, Pernal derived the general explicit form of the effective non-local potential for NOFT and analyzed its properties. ${ }^{45}$ Such a potential allows for the formulation of oneelectron equations similar to Hartree-Fock or DFT. Also, long before the advent of NOFT, Ludena had given a solid foundation for such an approach by proving the variational principle for the $n^{\text {th }}$-order reduced density matrix. ${ }^{46}$ In an attempt to develop functionals of better quality and reliability than DFT, Cioslowski introduced new constraints on the NOFs and examined the effect. ${ }^{47}$ Piris has recently developed a new, more complex NOF which has experienced more success than its earlier counterparts ${ }^{48-50}$ along with an iterative diagonalization method for determination of the natural orbitals. ${ }^{51}$ Other groups have also joined in the search for effective NOFs. ${ }^{52,53}$

Further removed from traditional methods, and even orbital functional methods, is the second-order reduced density matrix (2-RDM) approach. The 2-RDM solves the Schrödinger equation and can be used to calculate molecular properties without the need for a wave function. Mazziotti has developed two methods for solving the 2RDM problem, semidefinite programming and by solving the contracted Schrödinger equation. ${ }^{54}$ The methods have been shown to account for both dynamical and nondynamical correlation. However, while these methods do not approximate unknown correlation functionals, they do incorporate their own set of approximations. Currently, the solutions to the 2-RDM problem do not compare in efficiency to the meth- 
ods discussed previously. However, it is only the early stages of development.

The new concepts that have been introduced in recent years to deal with the correlation problem show promise. However, none have yet become sufficiently reliable and efficient to rival traditional methods. This implies a need for further development of current techniques and investigations into new strategies. The following sections present a possible approach to the correlation problem, its development and future path.

\section{A molecular orbital correlation functional}

The source of electron correlation is the approximation of the Hartree-Fock wave function, $\left|\Psi_{\mathrm{HF}}\right\rangle$, using a Slater determinant of spin orbitals, $\left\{\chi_{i}\right\}$. The corresponding expectation value of the electronic Hamiltonian, $\widehat{H}$ (Equation 6.2), or the HartreeFock energy, can be expressed over spin orbitals (Equation 6.23).

$$
E_{\mathrm{HF}}=\left\langle\Psi_{\mathrm{HF}}|\widehat{H}| \Psi_{\mathrm{HF}}\right\rangle=\sum_{i=1}^{N} h_{i i}+\frac{1}{2} \sum_{i=1}^{N} \sum_{j=1}^{N}\langle i j \| i j\rangle
$$

where $N$ is the number of electrons. The one-electron energy, $h_{i i}$, is given by the integral

$$
h_{i i}=\left\langle\chi_{i}|\hat{h}| \chi_{i}\right\rangle
$$

where $\hat{h}$ contains the kinetic energy and nuclear attraction potential energy operators.

$$
\hat{h}=-\frac{1}{2} \nabla_{1}^{2}-\sum_{A=1}^{M} \frac{Z_{A}}{r_{1 A}}
$$

where $M$ is the total number of nuclei. The second term of the Hartree-Fock energy expression is the two-electron energy, the Coulomb and exchange energy, given by the antisymmetrized two-electron integral, $\langle i j|| i j\rangle$.

$$
\langle i j|| i j\rangle=\langle i j \mid i j\rangle-\langle i j \mid j i\rangle
$$


The Coulomb, $J_{i j}$, and exchange, $K_{i j}$, integrals contain the electron-electron potential energy operator, $r_{12}^{-1}$.

$$
\begin{aligned}
& J_{i j}=\langle i j \mid i j\rangle=\iint \chi_{i}^{*}\left(\mathbf{x}_{1}\right) \chi_{j}^{*}\left(\mathbf{x}_{2}\right) \frac{1}{r_{12}} \chi_{i}\left(\mathbf{x}_{1}\right) \chi_{j}\left(\mathbf{x}_{2}\right) d \mathbf{x}_{1} d \mathbf{x}_{2} \\
& K_{i j}=\langle i j \mid j i\rangle=\iint \chi_{i}^{*}\left(\mathbf{x}_{1}\right) \chi_{j}^{*}\left(\mathbf{x}_{2}\right) \frac{1}{r_{12}} \chi_{j}\left(\mathbf{x}_{1}\right) \chi_{i}\left(\mathbf{x}_{2}\right) d \mathbf{x}_{1} d \mathbf{x}_{2}
\end{aligned}
$$

where $\mathbf{x}_{i}$ is the spatial and spin coordinates of the $i^{\text {th }}$ electron, $\mathbf{x}_{i}=\left(\mathbf{r}_{i}, \omega_{i}\right)$. The Hartree-Fock equations (Equation 6.29) are derived by minimization of the energy with respect to the spin orbitals under the constraint that they remain orthonormal, $\left\langle\chi_{i} \mid \chi_{j}\right\rangle=\delta_{i j}$, also known as the orbital Euler-Lagrange (OEL) method. ${ }^{55}$

$$
\hat{f} \chi_{i}\left(\mathbf{x}_{1}\right)=\epsilon_{i} \chi_{i}\left(\mathbf{x}_{1}\right)
$$

The Fock operator, $\hat{f}$, is given by

$$
\hat{f}=\hat{h}+\sum_{j=1}^{N} \hat{J}_{j}-\hat{K}_{j}
$$

where $\hat{J}_{j}$ and $\hat{K}_{j}$ are the Coulomb and exchange operators, respectively. The HartreeFock equations are solved by Roothaan's method (Equation 6.31), which involves expressing the Hartree-Fock equations over spatial, or molecular, orbitals, $\left\{\phi_{a}\right\}$. This is followed by expansion of the molecular orbitals (MOs) over basis functions, $\left\{\psi_{\mu}\right\}$, with MO coefficients, $\left\{C_{\mu a}\right\}$, and construction of a Fock matrix, F, which is diagonalized.

$$
\mathrm{FC}=\mathrm{SC} \epsilon
$$

where $\mathbf{S}$ is the overlap matrix, $\epsilon$ is the diagonal orbital energies matrix, and $\mathbf{C}$ is the matrix of MO coefficients. The Fock matrix depends on the MOs, and diagonalizing the Fock matrix yields MO coefficients. Therefore, the solution of the Hartree-Fock 
equations is iterative. The Fock operator acts independently on each electron, exposing it to the average field of the other electrons, referred to as a self-consistent field (SCF). The consequence of such an approach is the lack of electron correlation.

Could electron correlation be introduced if more flexibility was given to the HartreeFock energy expression? Such flexibility is introduced by allowing the occupancies of the spin orbitals, $n_{i}$, to vary between 0 and 1 . Electrons then not only occupy the first $N$ spin orbitals, but also have the freedom to occupy the $2 K-N$ virtual spin orbitals, where $K$ is the number of basis functions. In order to recover the density

$$
\rho(\mathbf{r})=\sum_{i=1}^{2 K} \chi_{i}^{\prime \prime}(\mathbf{x}) \chi_{i}^{\prime}(\mathbf{x})
$$

and agree with the Hartree-Fock energy expression when the first $N$ spin orbitals have occupancies of 1 , the spin orbitals take the form $\left\{\chi_{i}^{\prime}=\sqrt{n_{i}} \chi_{i}\right\}$, under the constraints, $0 \leq n_{i} \leq 1$ and $\sum_{i=1}^{2 K} n_{i}=N$. The resulting energy expression is a function of both the spin orbitals and the occupancies (Equation 6.33).

$$
E_{\mathrm{VOHF}}\left[n_{i}, \chi_{i}\right]=\sum_{i=1}^{2 K} n_{i} h_{i i}+\frac{1}{2} \sum_{i=1}^{2 K} \sum_{j=1}^{2 K} n_{i} n_{j}\left(J_{i j}-K_{i j}\right)
$$

The corresponding OEL equations, or variational occupancy Hartree-Fock (VOHF) equations, are

$$
n_{i} \hat{f}\left(n_{j}\right) \chi_{i}\left(\mathbf{x}_{1}\right)=\sum_{j=1}^{2 K} \epsilon_{j i} \chi_{j}\left(\mathbf{x}_{1}\right)
$$

where the Fock operator, $\hat{f}\left(n_{j}\right)$, depends on the occupancy.

$$
\hat{f}\left(n_{j}\right)=\hat{h}+\sum_{j=1}^{2 K} n_{j}\left(\hat{J}_{j}-\hat{K}_{j}\right)
$$

Unlike the Hartree-Fock case, minimization of the spin orbitals does not lead to a set of eigenvalue equations. The occupancy dependent Fock operator (Equation 6.34) is 
not invariant to a unitary transformation that diagonalizes the matrix of Lagrange multipliers, $\epsilon_{i j}$. Therefore, the resulting equations contain a sum of Lagrange multipliers (Equation 6.35). The energy is also a function of the occupancies, and hence a second set of equations are obtained by minimizing the energy with respect to the occupancies, under the constraint, $\sum_{i=1}^{2 K} n_{i}=N$.

$$
h_{i i}+\sum_{j=1}^{2 K} n_{j}\left(J_{i j}-K_{i j}\right)=\lambda
$$

When the above equation is compared to Equation 6.34 it is seen that $\lambda=\frac{\epsilon_{i i}}{n_{i}}$. In general, extremum may also be found at the endpoints of the interval containing the variational parameter, therefore Equation 6.36 need only be satisfied for $n_{i}$ such that $0<n_{i}<1$. Similar to Hartree-Fock theory the energy expression (Equation 6.33) and the orbital equations (Equation 6.34) can be expressed over spatial orbitals, $\phi_{a}$, by integration over the spin coordinate, $\omega$.

$$
\begin{gathered}
E_{\mathrm{VOHF}}\left[m_{a}, \phi_{a}\right]=\sum_{a=1}^{K} m_{a} h_{a a}+\frac{1}{2} \sum_{a=1}^{K} \sum_{b=1}^{K} m_{a} m_{b}\left(J_{a b}-\frac{1}{2} K_{a b}\right) \\
\frac{m_{a}}{2} \hat{f}\left(m_{b}\right) \phi_{a}\left(\mathbf{r}_{1}\right)=\sum_{b=1}^{K} \epsilon_{b a} \phi_{b}\left(\mathbf{r}_{1}\right)
\end{gathered}
$$

where the Fock operator over spatial orbitals is given by

$$
\hat{f}\left(m_{b}\right)=\hat{h}+\sum_{b=1}^{K} m_{b}\left(\hat{J}_{b}-\frac{1}{2} \hat{K}_{b}\right)
$$

For the closed-shell case, it is assumed that the alpha and beta occupancies of $\phi_{a}, n_{a}$ and $n_{\bar{a}}$, are equal and their sum is $m_{a}$. The allowed interval for the spatial orbital occupancy, $m_{a}$, is then $0 \leq m_{a} \leq 2$.

$$
n_{a}=n_{\bar{a}}
$$




$$
m_{a}=n_{a}+n_{\bar{a}}
$$

The OEL equations resulting from minimization of the energy with respect to occupancy become

$$
h_{a a}+\sum_{b=1}^{K} m_{b}\left(J_{a b}-\frac{1}{2} K_{a b}\right)=\lambda
$$

In order to determine the orbitals and their corresponding occupancies, the two sets of equations for spin orbitals (Equations 6.34 and 6.36) or spatial orbitals (Equations 6.38 and 6.42), must be solved simultaneously. However, first consider altering the Hartree-Fock occupancy in the closed-shell case (Equation 6.37) by transferring $\delta$ electrons from the occupied orbital $\phi_{a}$ to the unoccupied virtual orbital $\phi_{T}$. (i.e. $m_{a}: 2 \rightarrow 2-\delta$ and $\left.m_{r}: 0 \rightarrow \delta\right)$. The VOHF energy can be expressed in terms of $\delta$, $E_{\mathrm{VOHF}}[\delta]$.

$$
E_{\mathrm{VOHF}}[\delta]=E_{\mathrm{HF}}+\delta\left(\epsilon_{r}-\epsilon_{a}\right)+\frac{\delta^{2}}{4}\left(J_{a a}+J_{\tau r}\right)-\delta^{2}\left(J_{a r}-K_{a r}\right)
$$

The first term following the Hartree-Fock energy is a difference of orbital energies, which is always positive for Hartree-Fock orbitals, and the second is a sum of Coulomb energies, which are also positive. Therefore, it is difficult to imagine a situation in which,

$$
\left(\epsilon_{\tau}-\epsilon_{a}\right)+\frac{\delta}{4}\left(J_{a a}+J_{r \tau}\right)<\delta\left(J_{a r}-K_{a r}\right)
$$

While a rigorous proof of the opposite of the above inequality may be possible, it is not necessary. Lieb proved that an energy functional of the 1-RDM, with the form of the VOHF energy expression, is bounded from below by the Hartree-Fock energy. ${ }^{56}$

$$
E_{\mathrm{VOHF}} \geq E_{\mathrm{HF}}
$$


It can be concluded that the VOHF energy expression must take a different form if such an approach is to account for electron correlation. Actually, the VOHF energy expression, $E_{\mathrm{VOHF}}\left[n_{i}, \chi_{i}\right]$, closely resembles the NOFT, or 1-RDMFT, energy expressions. ${ }^{43}$ However, unlike NOFT, $E_{\mathrm{VOHF}}\left[n_{i}, \chi_{i}\right]$ does not include a correlation energy functional, $E_{\mathrm{corr}}\left[n_{i}, \chi_{i}\right]$. The derivation of a correlation functional is the next step in the development of such a theory.

Consider the closed-shell Hartree-Fock energy expression.

$$
E_{\mathrm{HF}}=2 \sum_{a=1}^{N / 2} h_{a a}+\sum_{a=1}^{N / 2} \sum_{b=1}^{N / 2} 2 J_{a b}-K_{a b}
$$

The energy expression can be modified, $E_{\mathrm{MHF}}$, by the addition of a correlation energy term.

$$
E_{\mathrm{MHF}}=2 \sum_{a=1}^{N / 2} h_{a a}+\sum_{a=1}^{N / 2} \sum_{b=1}^{N / 2} 2 J_{a b}-K_{a b}-\sum_{a=1}^{N / 2} \sum_{b=1}^{N / 2} Q_{a b}
$$

Derivation of the corresponding OEL equations results in a modified, correlated Fock operator.

$$
\hat{f}=\hat{h}+\sum_{b=1}^{N / 2} 2 \hat{J}_{b}-\hat{K}_{b}-\sum_{b=1}^{N / 2} \hat{q}_{b}
$$

The form of the correlation operator, $\hat{q}_{b}$, has important implications. If

$$
\left\langle a\left|\hat{q}_{b}\right| a\right\rangle=\langle a b|\hat{p}| a b\rangle
$$

where $\hat{p}$ does not depend on $\phi_{b}$, then

$$
\sum_{b=1}^{N / 2}\left\langle a\left|\hat{q}_{b}\right| a\right\rangle=\left\langle a\left|\frac{1}{2} \hat{q}(\rho)\right| a\right\rangle
$$

The correlation operator in this case, like the Coulomb operator, $\hat{J}_{b}$, may be written in terms of density, $\hat{q}[\rho(\mathbf{r})]$.

$$
\left\langle a\left|\hat{J}_{b}\right| a\right\rangle=\left\langle a b\left|r_{12}^{-1}\right| a b\right\rangle
$$




$$
\begin{aligned}
E_{\text {Coul }} & =\sum_{a=1}^{N / 2} \sum_{b=1}^{N / 2} 2 J_{a b} \\
& =2 \sum_{a=1}^{N / 2}\left\langle a\left|\sum_{b=1}^{N / 2} \hat{J}_{b}\right| a\right\rangle \\
& =2 \sum_{a=1}^{N / 2}\left\langle a\left|\frac{1}{2} \int \frac{\rho\left(r_{2}\right)}{r_{12}} d r_{2}\right| a\right\rangle \\
& =\frac{1}{2} \iint \frac{\rho\left(\mathbf{r}_{1}\right) \rho\left(\mathbf{r}_{2}\right)}{r_{12}} d \mathbf{r}_{1} d \mathbf{r}_{2}
\end{aligned}
$$

This implies the modified Hartree-Fock equations, using an operator of the form in Equation 6.49, are equivalent to the exact-exchange formulation of DFT. However, it is possible to define a correlation operator $\hat{p}_{b}$ that depends on $\phi_{b}$ then, unlike DFT, the operator and hence the functional operates at the MO level. One can also define a non-local correlation operator analogous to the exchange operator.

$$
\left\langle a\left|\hat{q}_{b}\right| a\right\rangle=\left\langle a b\left|\hat{p}_{b}\right| b a\right\rangle
$$

A correlation operator of this form differs from any DFT operator regardless of whether $\hat{p}$ depends on $\phi_{b}$ or not. The resulting modified Fock operator could then be used to solve for the modified, or correlated, Hartree-Fock orbitals using Roothaan's equation (Equation 6.31). This approach resembles exact-exchange DFT, however the operator now acts at the MO level and may be local or non-local. However, if a MHF method cannot accurately account for electron correlation, variational occupancy may be introduced to the correlation operator, $\hat{q}_{b}\left(m_{b}\right)$, along with the entire energy expression, as in VOHF (Equation 6.37). The resulting energy expression is then analogous to that of NOFT, or 1-RDMFT.

$$
E_{\mathrm{NOFT}}\left[\phi_{a}, m_{a}\right]=E_{\mathrm{VOHF}}\left[\phi_{a}, m_{a}\right]-Q\left[\phi_{a}, m_{a}\right]
$$


As previously mentioned, NOFT has experienced some success in dealing with the correlation problem but is currently far from becoming a mainstream computational chemistry method. There is a need for the development of accurate and universal correlation functionals. It is important that the new correlation functionals treat simple systems, such as two and four electron systems correctly, as fundamental flaws may lead to unreliability.

For a two-electron singlet system there is Coulomb correlation, but no Fermi correlation, hence no exchange. For a two-electron triplet system, there is Fermi correlation, or exchange, and, as a result, minimal Coulomb correlation. Curiosity then leads to a comparison of the Coulomb correlation of the singlet to the Fermi correlation of the triplet (Figure 6.3). The exchange energy of the triplet systems was calculated at UHF using a scaled aug-cc-pVQZ basis set for helium.

The comparison of the correlation energy of the two-electron singlet system to the exchange energy of the triplet as function of nuclear charge, $Z$, reveals no obvious relationship. Although, it is noted that the correlation and exchange energies for helium are quite close, -0.042044 hartrees and -0.039312 hartrees, respectively.

The different behaviours of the correlation energy as a function of $Z$ for twoelectron and four-electron systems (Figure 6.1), is a fundamental property that should be modelled by a correlation energy functional and corresponding correlation operator. The linear behaviour of the four-electron systems is due to the near degeneracy of the $2 \mathrm{~s}$ and $2 \mathrm{p}$ atomic orbitals. This suggests a connection between the correlation energy and the HOMO-LUMO gap. The HOMO-LUMO gap, calculated at HF/6$31 \mathrm{G}(\mathrm{d})$, for two and four-electron systems as a function of $Z$ is given in Figure 6.4.

The HOMO-LUMO gap for two-electron systems is found to be a quadratic in 


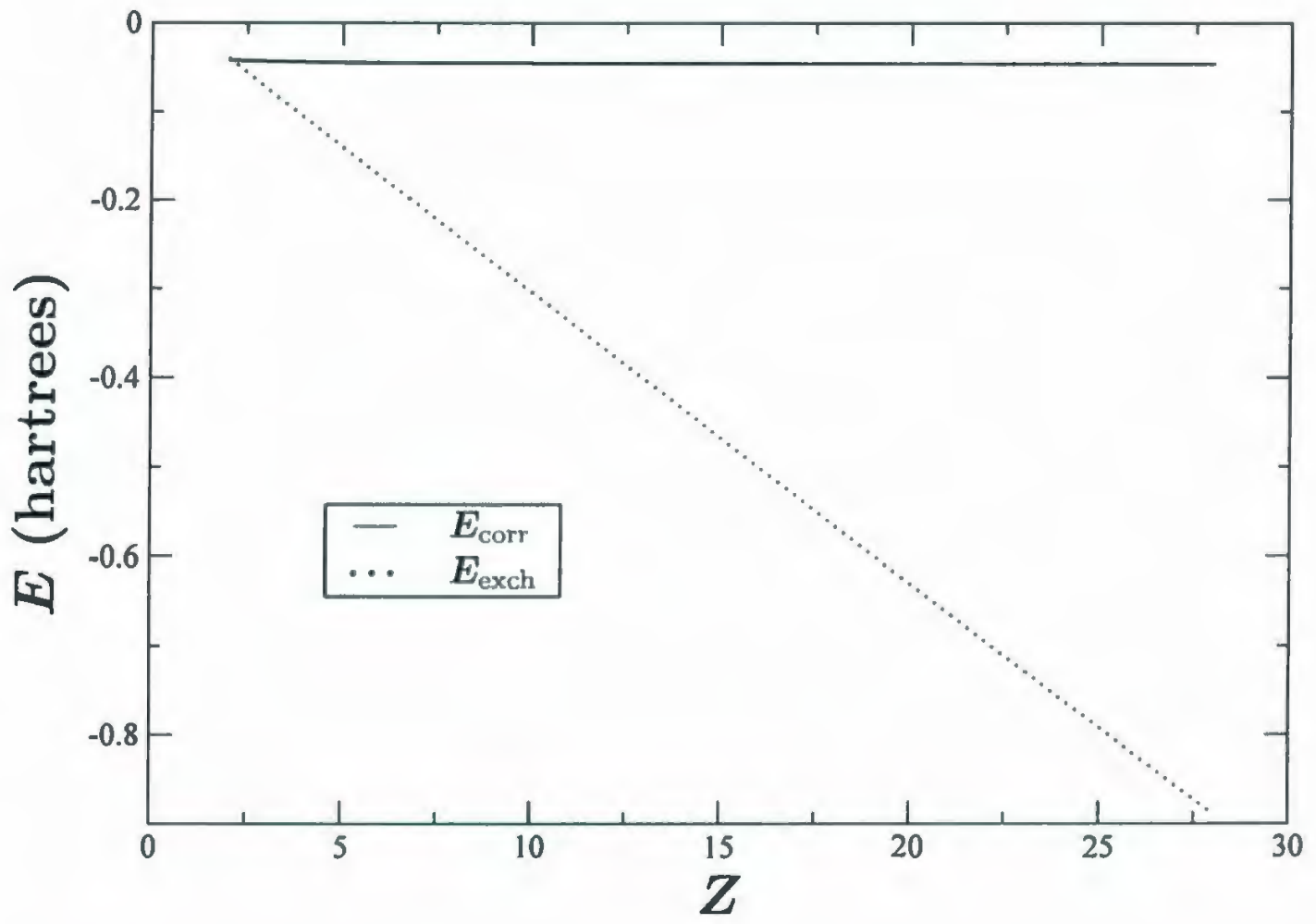

Figure 6.3. Exact correlation energy of two-electron singlet systems compared to exchange energy of two-electron triplet systems, over a range of nuclear charge, $Z=2-28$. Exchange energy calculated at UHF using a scaled aug-cc-pVQZ helium basis set. 


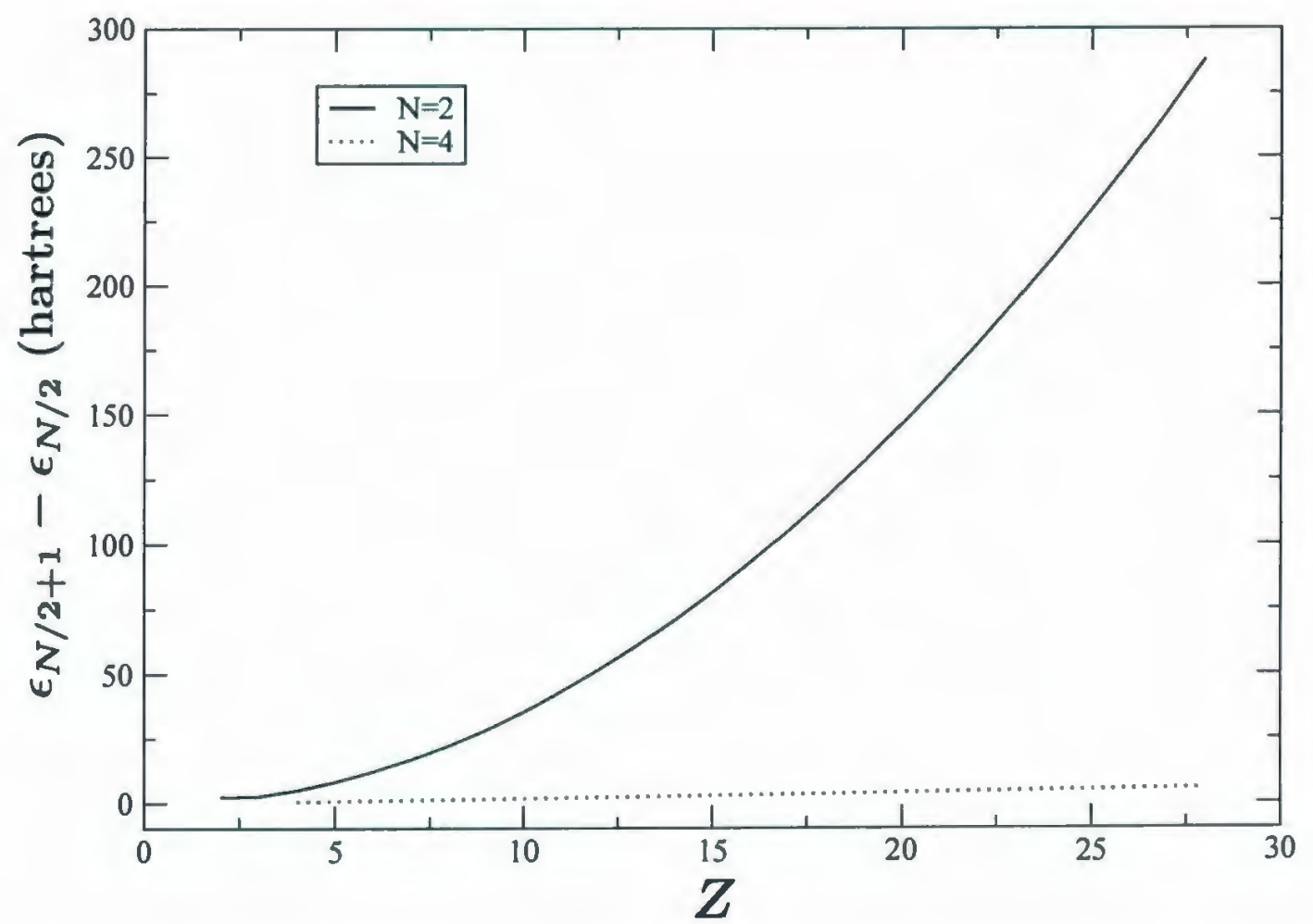

Figure 6.4. HOMO-LUMO gap of two and four-electron systems as a function of nuclear charge, Z.(HF/6-31G(d)) 
$Z$, whereas for four electrons, it is linear in $Z$. This power of $Z$ difference is the inverse of that seen for the correlation energy. Therefore, a functional which consists of the inverse of the HOMO-LUMO gap multiplied by a functional which is linear in $Z$ for both systems, $\Gamma\left[\phi_{N / 2}, \phi_{N / 2+1}, \ldots\right]$, will at least qualitatively model the different behaviours of the correlation energy for two and four-electron systems.

$$
Q\left[\phi_{a}\right]=\frac{\Gamma\left[\phi_{N / 2}, \phi_{N / 2+1}, \ldots\right]}{\epsilon_{N / 2+1}-\epsilon_{N / 2}}
$$

Upon investigation of some MO properties, it was found that the HOMO energy is an approximately linear function of $Z$. A plot of the $Q\left[\phi_{a}\right]$ as a function of $Z$ for two and four-electron systems, where $\Gamma\left[\phi_{N / 2}, \phi_{N / 2+1}, \ldots\right]=\epsilon_{N / 2}$, is given in Figure 6.5.

It is noticed that $Q\left[\phi_{a}\right]$ is about two orders of magnitude larger than that of the correlation energy, however at this point we only illustrate the ability to qualitatively model the behaviours of correlation energy for two and four-electron systems in the high density limit. Howard and March have actually suggested that an exchangecorrelation functional could be expressed solely in terms of HOMO and LUMO properties. ${ }^{57}$ It is not necessarily suggested here that a correlation functional should be derived from HOMO and LUMO energies. However, it is suggested that a functional should be constructed from MO properties. There has been previous success in relating the size of localized molecular orbitals (LMOs) to the correlation energy of small molecules. ${ }^{58}$ The recently derived average distance between two electrons ${ }^{59}$ is a generalization of LMO size and could possibly be used to construct a correlation energy functional. Other useful properties may include kinetic and potential energies, and first, second and even higher order moments. However, an alternative method for discovering an accurate correlation energy functional is the investigation of correlated 


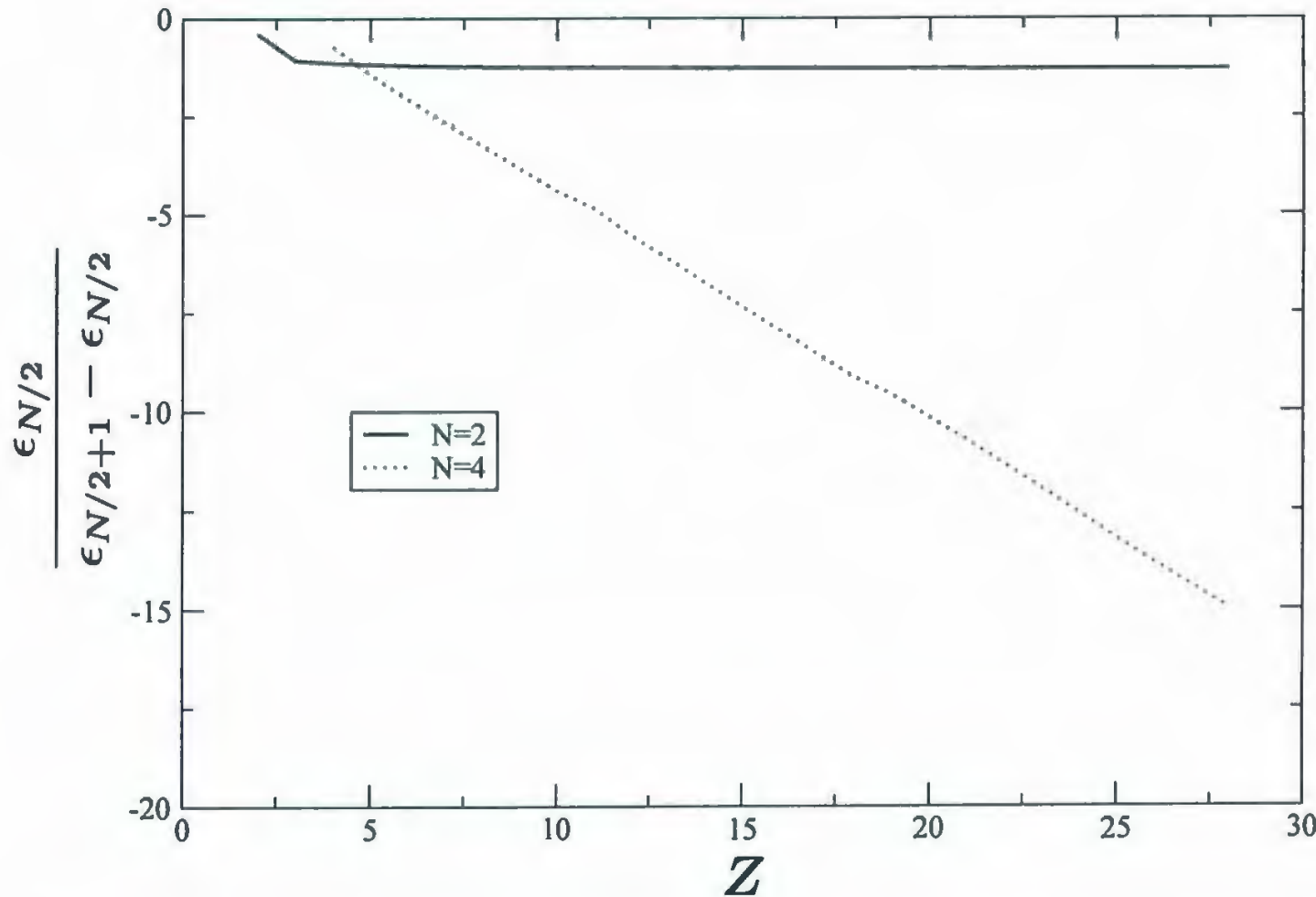

Figure 6.5. Function of HOMO-LUMO energies for two and four-electron systems as a function of nuclear charge, $Z$. (HF/6-31G(d)) 
post Hartree-Fock energy expressions.

\section{The explicit CISD energy expression}

Insight into the required elements of a $\mathrm{MO}$ correlation energy functional can be obtained through examination of the CI energy expressions. Consider, the very simple, double CI (DCI) wave function for He with two basis functions (i.e one virtual).

$$
\left|\Psi_{\mathrm{DCI}}(\mathrm{He})\right\rangle=c_{0}\left|\Psi_{0}\right\rangle+c_{1 \overline{1}}^{2 \overline{2}}\left|\Psi_{1 \overline{1}}^{2 \overline{2}}\right\rangle
$$

The wave function is a sum of the Hartree-Fock wave function, $\left|\Psi_{0}\right\rangle$, and the deter-

minant describing the double excitation, $\left|\Psi_{1 \overline{1}}^{2 \overline{2}}\right\rangle$. The DCI energy expression is derived from the expectation value of the Hamiltonian with the DCI wave function.

$$
E_{\mathrm{DCI}}(\mathrm{He})=c_{0}^{2} E_{0}+\left(c_{1 \overline{1}}^{2 \overline{2}}\right)^{2} E_{1 \overline{\mathrm{I}}}^{2 \overline{2}}+2 c_{0} c_{1 \overline{1}}^{2 \overline{2}} K_{12}
$$

The first two terms are contributions from the energies of each configuration,

$$
\begin{gathered}
E_{0}=2 h_{11}+J_{11} \\
E_{1 \overline{1}}^{2 \overline{2}}=2 h_{22}+J_{22}
\end{gathered}
$$

Mixing of the Hartree-Fock wave function with the double excitation gives an exchange contribution, $c_{0} c_{1 \overline{1}}^{2 \overline{2}} K_{12}$. While the helium DCI wave function with two basis functions only recovers a tiny fraction of the total correlation energy, the mechanism for lowering the energy is of interest. An increase of the contribution from the double excitation energy will only raise the energy. However, this is counteracted by the increase in contribution from the exchange integral, $K_{12}$, which lowers the energy. Actually, the above DCI equation is analogous to the natural expansion energy 
expression for two electron systems, $E_{\mathrm{NE}}{ }^{60}$

$$
E_{\mathrm{NE}}=\sum_{i=1}^{K} \gamma_{i}^{2} h_{i i}+\sum_{i=1}^{K} \sum_{j=1}^{K} \gamma_{i} \gamma_{j} K_{i j}
$$

For two basis function DCI(Equation 6.57) or equivalently two natural orbitals in the natural expansion (Equation 6.60), the expressions become identical for $\gamma_{1}=c_{0}$ and $\gamma_{2}=c_{1 \overline{1}}^{2 \overline{2}}$. If more basis functions, or natural orbitals, are added, the expressions remain equivalent if the DCI wave function includes only excitations of the form $\left|\Psi_{1 \overline{1}}^{r \bar{T}}\right\rangle$ (i.e. to the same spatial orbital). However, in the case of the DCI equations, it is only the coefficients, $\left\{c_{i}\right\}$, that are variational. Whereas, in the natural expansion, both the orbitals and the coefficients, $\left\{\gamma_{i}\right\}$, are variational. If a MCSCF approach was applied to the modified DCI wave function, then the natural orbitals would be the solution. In the case of the natural expansion, two sets of OEL equations can be derived, corresponding to minimization of the energy with respect to the natural orbitals and the coefficients. ${ }^{60}$ The OEL equations are similar to those derived previously for VOHF (Equations 6.34 and 6.36). However, no attempt has been made to solve the equations for the natural orbitals. ${ }^{60}$ Kutzelnigg did solve approximate versions of the equations, yet they only apply to two-electron systems. Others have determined the natural orbitals for two-electron systems using other methods in an effort to explore the effects of electron correlation. ${ }^{60}$ While insight may be gained from the various wave functions and energy expressions for two-electron systems, a similar investigation could be extended to many-electron systems.

The general CISD wave function, in terms of alpha and beta spin orbitals, is given by

$$
\left|\Psi_{\mathrm{CISD}}\right\rangle=c_{0}\left|\Psi_{0}\right\rangle+\sum_{a r} c_{a}^{r}\left|\Psi_{a}^{r}\right\rangle+\sum_{a r} c_{\bar{a}}^{\bar{r}}\left|\Psi_{\bar{a}}^{\bar{r}}\right\rangle
$$




$$
+\frac{1}{4} \sum_{a b r s} c_{a b}^{r s}\left|\Psi_{a b}^{r s}\right\rangle+\frac{1}{4} \sum_{a b r s} c_{\bar{a} \bar{b}}^{\bar{r} \bar{s}}\left|\Psi_{\bar{a} \bar{b}}^{\bar{r} \bar{s}}\right\rangle+\sum_{a b r s} c_{a}^{r \bar{s}}\left|\Psi_{a \bar{b}}^{r \bar{s}}\right\rangle
$$

The wave function can be expressed as the sum of the Hartree-Fock wave function, $\left|\Psi_{0}\right\rangle$, and determinants describing single alpha excitations, $\left|\Psi_{a}^{r}\right\rangle$, single beta excitations, $\left|\Psi_{\bar{a}}^{\bar{r}}\right\rangle$, double alpha excitations, $\left|\Psi_{a b}^{r s}\right\rangle$, double beta excitations, $\left|\Psi_{\bar{a} \bar{b}}^{\overline{r s}}\right\rangle$, and alpha-beta excitations, $\left|\Psi_{a \bar{b}}^{r \bar{s}}\right\rangle$. The energy expression is calculated as the expectation value of the Hamiltonian.

$$
E_{\mathrm{CISD}}=\left\langle\Psi_{\mathrm{CISD}}|\widehat{H}| \Psi_{\mathrm{CISD}}\right\rangle
$$

The energy expression over spatial orbitals can be derived using the Slater-Condon rules for matrix element evaluation. ${ }^{18}$ Also, each possible combination of alpha and beta spin orbitals must be accounted for in the derivation. For example, the matrix element $\left\langle\Psi_{a}^{r}|\widehat{H}| \Psi_{b}^{s}\right\rangle$, has four possible cases; $a \neq b$ and $r \neq s, a=b$ and $r \neq s, a \neq b$ and $r=s$, and $a=b$ and $r=s$. To include each case in the general expanded energy expression, each term is multiplied by the corresponding conditional function. The matrix element for the $a \neq b$ and $r \neq s$ case is multiplied by $\left(1-\delta_{a b}\right)\left(1-\delta_{r s}\right)$, an and statement. The cases, $a=b$ and $r \neq s$, and $a \neq b$ and $r=s$, are multiplied by $\delta_{a b}\left(1-\delta_{r s}\right)$ and $\delta_{r s}\left(1-\delta_{a b}\right)$, respectively. The conditional function for the case $a=b$ and $r=s$, is of course $\delta_{a b} \delta_{r s}$. Similar to the and statement, an or statement could be constructed if necessary, for the case $a \neq b$ or $r \neq s$. The conditional function would be $\left(1-\delta_{a b} \delta_{r s}\right)$. Simplification of the energy expression is achieved by expressing matrix elements of configurations that differ by one spin orbital, in terms of off-diagonal Fock matrix elements, $F_{i j}, i \neq j$. For example, the matrix element 
$\left\langle\Psi_{a}^{r}|\widehat{H}| \Psi_{a}^{s}\right\rangle$ expanded using the Slater-Condon rules gives

$$
\left\langle\Psi_{a}^{r}|\widehat{H}| \Psi_{a}^{s}\right\rangle=h_{r s}+\sum_{p}\langle r p \| s p\rangle
$$

where the sum over $p$ includes all the spin orbitals of either $\left|\Psi_{a}^{r}\right\rangle$, or $\left|\Psi_{a}^{s}\right\rangle$. It is noticed that the matrix element closely resembles an off-diagonal Fock matrix element, $F_{r s}$.

$$
h_{r s}+\sum_{p}\langle r p \| s p\rangle=F_{r s}-\langle r a \| s a\rangle
$$

Substitution using the above equality leads to significant simplification of the general CISD energy expression and the presence of Fock matrix elements leads to easier evaluation of such an energy expression. However, in the derivation presented here, the MOs are considered to be fixed Hartree-Fock MOs and therefore $F_{i j}=0, i \neq$ j. The general CISD closed-shell energy expression is derived using the conditional functions, the Slater-Condon rules, the orthogonality of the spin functions, and the off-diagonal Fock matrix element substitution.

$$
\begin{gathered}
E_{\mathrm{CISD}}=E_{0}+2 \sum_{a r}\left(c_{a}^{r}\right)^{2}\left(\epsilon_{r}-\epsilon_{a}\right)+\sum_{a b r s}\left\{\frac{1}{2}\left(c_{a b}^{r s}\right)^{2}+\left(c_{a \bar{b}}^{r \bar{s}}\right)^{2}\right\}\left(\epsilon_{r}+\epsilon_{s}-\epsilon_{a}-\epsilon_{b}\right) \\
+\sum_{a b r s}\left(D_{a b r s}\langle a b \mid r s\rangle-D_{a r b s}\langle a r \mid b s\rangle\right)+\sum_{a r s t} D_{a r s t}\langle a r \mid s t\rangle+\sum_{a b c r} D_{a b c r}\langle a b \mid c r\rangle \\
+\sum_{r s t u} D_{r s t u}\langle r s \mid t u\rangle+\sum_{a b c d} D_{a b c d}\langle a b \mid c d\rangle \\
+\sum_{a r}\left\{\sum_{b s}\left(c_{b \bar{a}}^{s \bar{r}}\right)^{2}+\left(c_{a \bar{b}}^{s \bar{r}}\right)^{2}+\left(c_{b \bar{a}}^{r \bar{s}}\right)^{2}-3\left(c_{a \bar{b}}^{r \bar{s}}\right)^{2}\right\} J_{a r} \\
+\sum_{a r}\left\{\sum_{b s}\left(c_{a \bar{b}}^{r \bar{s}}\right)^{2}-\left(c_{b \bar{a}}^{s \bar{T}}\right)^{2}\right\} K_{a r}
\end{gathered}
$$

where

$$
D_{a b r s}=2 c_{0}\left\{c_{a b}^{r s}+c_{a \bar{b}}^{r \bar{s}}\right\}+4 c_{a}^{r} c_{b}^{s}+\sum_{c t}\left\{2 c_{c a}^{t r} c_{c b}^{t s}+2 c_{c a}^{t r} c_{c \bar{b}}^{t \bar{s}}+2 c_{c a}^{t r} c_{b \bar{c}}^{s \bar{t}}+c_{c \bar{a}}^{t \bar{r}} c_{c \bar{b}}^{t \bar{s}}+c_{a \bar{c}}^{r \bar{t}} c_{b \bar{c}}^{s \bar{t}}\right\}
$$




$$
\begin{gathered}
D_{a r b s}=2 c_{a}^{r} c_{b}^{s}+\sum_{c t}\left\{2 c_{a c}^{t r} c_{c b}^{t s}+c_{c \bar{a}}^{t \bar{r}} c_{c \bar{b}}^{t \bar{s}}+c_{a \bar{c}}^{r \bar{t}} c_{b \bar{c}}^{s \overline{\bar{c}}}+c_{c \bar{a}}^{r \overline{\bar{t}}} c_{c \bar{b}}^{s \overline{\bar{b}}}+c_{a \bar{c}}^{t \bar{r}} c_{b \bar{c}}^{t \bar{s}}\right\} \\
D_{a r s t}=\sum_{b} c_{b}^{r}\left\{4 c_{a b}^{s t}+2 c_{b \bar{a}}^{t \bar{s}}+2 c_{a \bar{b}}^{s \overline{\bar{t}}}\right\} \\
D_{a b c r}=\sum_{s} c_{c}^{s}\left\{4 c_{a b}^{r s}-2 c_{a \bar{b}}^{s \overline{\bar{T}}}-2 c_{b \bar{a}}^{r \overline{\bar{s}}}\right\} \\
D_{r s t u}=\sum_{a b}\left\{\frac{1}{2} c_{a b}^{r s} c_{a b}^{t u}+c_{a b}^{r \bar{s}} c_{a \bar{b}}^{t \bar{u}}\right\} \\
D_{a b c d}=\sum_{r s}\left\{\frac{1}{2} c_{a b}^{r s} c_{c d}^{r s}+c_{a \bar{b}}^{r \bar{s}} c_{c \bar{d}}^{r \bar{s}}\right\}
\end{gathered}
$$

Also important for simplification, is the antisymmetry of the coefficients, $c_{a b}^{r s}=-c_{a b}^{s r}=$ $-c_{b a}^{r s}=c_{b a}^{s r}$. In the closed-shell case, there are also the relations, $c_{a}^{r}=c_{\bar{a}}^{\bar{r}}, c_{a b}^{r s}=c_{\bar{a} \bar{b}}^{\overline{r s}}$, and $c_{a \bar{b}}^{r \bar{s}}=c_{b \bar{a}}^{s \bar{r}}$. Therefore, the general closed-shell CISD energy is given in terms of single excitations, $c_{a}^{r}$, double excitations of the same spin, $c_{a b}^{r s}$, and double excitations of opposite spin, $c_{a \bar{b}}^{r \bar{s}}$. In principle, the CISD wave function could be determined first, by determination of the Hartree-Fock MOs and then, by minimization of the CISD energy expression (Equation 6.65) using some optimization technique such as Newton-Raphson. Solving the CISD equation using an optimization technique avoids the usual matrix diagonalization associated with solving the CI equations, which is normally done iteratively by Davidson's method. ${ }^{61}$ While there may not necessarily be any speed advantages by solving the CISD problem using Equation 6.65 , it does allow for the observation of which terms are important for electron correlation. These observations could lead to approximate equations by truncation as well as correlation functionals which model the effects of the important correlation energy terms. Alternatively, OEL equations could be derived with respect to the orbitals and possibly the coefficients, which leads to another method of solving the CISD problem. Such an approach would be similar to the correlated one-particle (COP) method of Bartlett et 
al. in which a one-particle operator is derived from the coupled-cluster equations. ${ }^{62}$

\section{Conclusions}

The correlation problem continues to plague computational chemists while providing a very interesting challenge to theoreticians. The methods of dealing with the problem are continually evolving, with the appearance of exact-exchange density functional theory, natural orbital, or first-order reduced density matrix, functional theory, and second-order reduced density matrix methods which require no wave function or density. It is not obvious which approach may lead to the most effective method for dealing with electron correlation. However, it is believed that better results will be achieved by considering the quantum nature of the electrons, in other words, their orbitals, rather than a classical density distribution.

Orbital functional theory is in the early stages of development and moderate success has been achieved for some natural orbital functionals. The number of different NOFs is quite small. Investigations into the relationships between correlation energy and MO properties is an important tool for the development of an effective MO correlation functional. The correlation energy obtained by post Hartree-Fock methods can be expressed in terms of integrals over MOs which, of course, are also MO properties. The form of such properties and their behaviour also provides insight into how to derive a $\mathrm{MO}$ correlation energy functional.

\section{Acknowledgements}

The authors would like to thank the National Sciences and Engineering Research Council of Canada (NSERC) for funding and the Atlantic Computational Excellence 
Network (ACENet) for computational resources. 


\section{References}

(1) K. Raghavachari and J. B. Anderson. J. Phys. Chem., 100:12960-12973, 1996.

(2) D. P. Tew, W. Klopper, and T. Helgaker. J. Comp. Chem., 28:1307-1320, 2007.

(3) D. K. W. Mok, R. Neumann, and N. C. Handy. J. Phys. Chem., 100:6225-6230, 1996.

(4) E. Clementi and G. Corongiu. J. Mol. Struct., 543:39-52, 2001.

(5) E. A. Hylleraas. Z. Physik A, 54:347-366, 1929.

(6) C. Amovilli, N. H. March, I. A. Howard, and A. Nagy. Phys. Lett. A, 372:4053$4056,2008$.

(7) E. Buendia, F.J. Gálvez, and A. Sarsa. Chem. Phys. Lett., 428:241-244, 2006.

(8) E. A. Hylleraas. Z. Physik A, 65:209-225, 1930.

(9) E. R. Davidson, S. A. Hagstrom, S. Chakravorty, V. Meiser Umar, and Charlotte Froese Fischer. Phys. Rev. A, 44:7071-7083, 1991.

(10) S. Chakravorty, S. R. Gwaltney, E. R. Davidson, F. A. Parpia, and C. Froese Fischer. Phys. Rev. A, 47:3649-3670, 1993.

(11) P. M. W. Gill and D. P. O'Neill. J. Chem. Phys., 122:094110, 2005.

(12) C. D. Sherrill and H. F. Schaefer III. Adv. Quant. Chem., 34:143-269, 1999.

(13) B. Roos. Chem. Phys. Lett., 15:153-159, 1972.

(14) P. J. Knowles and N. C. Handy. Chem. Phys. Lett., 111:315-321, 1984. 
(15) Z. Rolik, A. Szabados, and P. R. Surján. J. Chem. Phys., 128:144101, 2008.

(16) J. A. Pople, M. Head-Gordon, and K. Raghavachari. J. Chem. Phys., 87:5968$5975,1987$.

(17) T. J. Lee, A. P. Rendell, and P. R. Taylor. J. Phys. Chem., 94:5463-5468, 1990.

(18) A. Szabo and N. S. Ostlund. Modern Quantum Chemisty: Introduction to Advanced Electronic Structure Theory. Dover Publications, Inc., first edition, 1996.

(19) W. Klopper and W. Kutzelnigg. J. Phys. Chem., 94:5625-5630, 1990.

(20) W. Klopper and W. Kutzelnigg. J. Chem. Phys., 94:1985-2001, 1991.

(21) J. Noga and W. Kutzelnigg. J. Chem. Phys., 101:7738-7762, 1994.

(22) M. W. Schmidt and M. S. Gordon. Annu. Rev. Phys. Chem., 49:233-266, 1998.

(23) H.-J. Werner and W. Meyer. J. Chem. Phys., 73:2342-2356, 1980.

(24) W. Koch and M. C. Holthausen. A Chemist's Guide to Density Functional Theory. Wiley-VCH, second edition, 2001.

(25) A. D. Becke. Phys. Rev. A, 38:3098-3100, 1988.

(26) C. Lee, W. Yang, and R. G. Parr. Phys. Rev. B, 37:785-789, 1988.

(27) A. D. Becke and E. R. Johnson. J. Chem. Phys., 127:124108, 2007.

(28) Y. Zhao and D. G. Truhlar. Theo. Chem. Acc., 120:215-241, 2008.

(29) A. Savin. Chem. Phys., 356:91-97, 2009. 
(30) V. N. Staroverov, G. E. Scuseria, J. P. Perdew, and J. Tao. Phys. Rev. A, 70:012502, 2004 .

(31) J. Katriel, M. Bauer, M. Springborg, S. P. McCarthy, and A. J. Thakkar. J. Chem. Phys., 127:024101, 2007.

(32) A. J. Cohen, P. Mori-Sánchez, and W. Yang. J. Chem. Phys., 126:191109, 2007.

(33) P. M. W. Gill, D. L. Crittenden, D. O'Neill, and N. A. Besley. Phys. Chem. Chem. Phys., 8:15-25, 2006.

(34) P. Gori-Giorgi and A. Savin. Phys. Rev. A, 71:032513, 2005.

(35) P. Gori-Giorgi and A. Savin. J. Chem. Theory Comput., 3:796-802, 2007.

(36) P. Gori-Giorgi and A. Savin. Int. J. Quant. Chem., 109:1950-1961, 2009.

(37) R. Fondermann, M. Hanrath, and M. Dolg. Theo. Chem. Acc., 118:777-783, 2007.

(38) V. A. Rassolov. J. Chem. Phys., 110:3672-3677, 1999.

(39) J. K. Pearson, D. L. Crittenden, and P. M. W. Gill. J. Chem. Phys., 130:164110, 2009.

(40) R. K. Nesbet. Int. J. Quant. Chem., 85:405-410, 2001.

(41) R. K. Nesbet. Int. J. Quant. Chem., 102:602-605, 2005.

(42) S. Goedecker and C. J. Umrigar. Phys. Rev. Lett., 81:866-869, 1998.

(43) V. N. Staroverov and G. E. Scuseria. J. Chem. Phys., 117:2489-2495, 2002. 
(44) A. J. Cohen and E. J. Baerends. Chem. Phys. Lett., 364:409-419, 2002.

(45) K. Pernal. Phys. Rev. Lett., 94:233002, 2005.

(46) E. V. Ludena. J. Mol. Struct., 123:371-382, 1985.

(47) J. Cioslowski. J. Chem. Phys., 123:164106, 2005.

(48) P. Leiva and M. Piris. J. Mol. Struct., 719:63-67, 2005.

(49) P. Leiva and M. Piris. J. Mol. Struct., 770:45-49, 2006.

(50) P. Leiva and M. Piris. Int. J. Quant. Chem., 107:1-11, 2007.

(51) M. Piris and J. M. Ugalde. J. Comp. Chem., early view, 2009.

(52) N. N. Lathiotakis, N. Helbig, A. Zacarias, and E. K. U. Gross. J. Chem. Phys., 130:064109, 2009.

(53) D. R. Rohr, K. Pernal, O. V. Gritsenko, and E. J. Baerends. J. Chem. Phys., 129:164105, 2008.

(54) D. A. Mazziotti. Acc. Chem. Res., 39:207-215, 2006.

(55) R. K. Nesbet. Phys. Rev. A, 62:040701, 2000.

(56) E. H. Lieb. Phys. Rev. Lett., 46:457-459, 1981.

(57) I. A. Howard and N. H. March. Chem. Phys. Lett., 402:1-3, 2005.

(58) M. R. Peterson, R. A. Poirier, R. Daudel, and I. G. Csizmadia. Int. J. Quant. Chem., 19:25-31, 1981. 
(59) J. W. Hollett and R. A. Poirier. J. Theoretical Computational Chem., 6:13-22, 2007.

(60) E. R. Davidson. Reduced Density Matrices in Quantum Chemistry. Academic Press, Inc., first edition, 1976.

(61) E. R. Davidson. J. Comput. Phys., 17:87-94, 1975.

(62) A. Beste and R. J. Bartlett. J. Chem. Phys., 123:154103, 2005. 


\section{Chapter 7}

\section{Summary}

In general, theoretical chemistry is aimed towards making computational methods faster and more accurate. However, with such a range of computational methods, from full CI to molecular mechanics, there are many ways to increase efficiency. Simplification of information included in molecular wave functions, that allows for easier interpretation, provides a means to determine steric interactions without the calculation of reaction energetics (Chapter 2). If the focus is narrowed to electronelectron and electron-nucleus interactions, a similar sort of simplification not only models the interactions (Chapters 3 and 4 ) but leads to a theory that bridges the gap between electronic structure and molecular mechanics (Chapter 5). It is also possible that similar modelling approaches can lead to faster, more accurate, wave function methods (Chapter 6).

The theoretical definition of the size and shape of a molecule or substituent (Chapter 2) correlates well with more sophisticated theoretical methods of determining molecular volume and can predict steric effects. However, no use has been made of the shape components provided by the origin invariant second moment tensor. It is 
expected that for sterically hindered reactions in which orientation plays a role, the shape of the substituent may provide insight into experimental results. The shape and size of a substituent could also be incorporated into conformational search algorithms such as those used for proteins.

The relationships between the average interelectronic distance (Chapters 3 and 4) and average electron-nucleus distance (Chapter 4) and the corresponding molecular orbital energy components have already led to the development of SEST (Chapter 5). However, the relationships observed are not exact, and the investigation of the deviations, such as the delocalized core molecular orbitals, will lead to a better understanding of molecular orbital properties and possibly more accurate models. Furthermore, the development of SEST is not the only application of these relationships. The approximation of a two-electron property, the Coulomb integral, by a one-electron property, the average interelectronic distance, is an important result that could be used to speed up large SCF calculations or geometry optimizations. Also, as previously suggested, the average interelectronic distance may also be useful in the development of a molecular orbital correlation functional.

Simulated Electronic Structure Theory (SEST) is in the early stages of its development (Chapter 5). There are many fundamental aspects of the theory that need to be addressed, such as the transferability of the atomic interactions and the universality of the functional form. Investigations into such issues will likely require the construction of a small force field for a collection of small molecules for which such properties as vibrational frequencies and bond energetics could be calculated. In order to construct a force field, an algorithm for determining the parameters of a set of molecules will also be needed. There is still a significant amount of work to be 
done before SEST is applicable to large chemical systems like molecular mechanics. However, the advantages of including the electronic structure should provide some incentive.

In observing the recent trend in new approaches to the correlation problem (Chapter 6), the development of orbital functional theory appears inevitable. One arrives at a similar conclusion by starting at Hartree-Fock theory and searching for ways to include the electron correlation. The challenge is then to derive an efficient electron correlation functional in terms of molecular orbitals. There may be several ways to achieve this goal including investigation of explicit energy expressions of correlated wave functions and the relationship between the correlation energy and properties of molecular orbitals.

The use of first and second moment operators to derive properties of molecular orbitals and molecular wave functions has led to significant developments across different areas of theoretical chemistry. The simple models that have been derived lead to very useful approximations while providing a mechanism for interpretation. Two important avenues of future research include two somewhat different theories. Both SEST and molecular orbital functional theory could have a significant impact on the future of computational chemistry. 


\section{Appendix A}

\section{Density Functionals}

Local Spin Density Approximation(LSDA) exchange energy functional ${ }^{1}$

$$
E_{x}^{\mathrm{LSDA}}=-\frac{3}{4}\left(\frac{6}{\pi}\right)^{1 / 3} \int\left[\rho_{\alpha}(\mathbf{r})^{4 / 3}+\rho_{\beta}(\mathbf{r})^{4 / 3}\right] d \mathbf{r}
$$

where $\rho_{\alpha}(\mathbf{r})$ and $\rho_{\beta}(\mathbf{r})$ are the $\alpha$ and $\beta$ spin densities.

Becke's 1988 exchange energy functional (B88 or B $)^{2}$

$$
E_{x}^{\mathrm{B} 88}=E_{x}^{\mathrm{LSDA}}-b \sum_{\sigma=\alpha, \beta} \int \frac{\rho_{\sigma}(\mathbf{r})^{4 / 3} \chi_{\sigma}^{2}}{1+6 b \chi_{\sigma} \sinh ^{-1} \chi_{\sigma}} d \mathbf{r}
$$

where $b=0.0042$ a.u. and $\chi_{\sigma}$ is the reduced density gradient for spin $\sigma$.

$$
\chi_{\sigma}=\frac{\left|\nabla \rho_{\sigma}(\mathbf{r})\right|}{\rho_{\sigma}(\mathbf{r})^{4 / 3}}
$$




\section{Lee-Yang-Parr correlation energy functional (LYP $)^{3}$}

$$
\begin{aligned}
E_{c}^{\mathrm{LYP}} & =-a \int \frac{\gamma(\mathbf{r})}{1+d \rho(\mathbf{r})^{-1 / 3}}\left\{\rho(\mathbf{r})+2 b \rho(\mathbf{r})^{-5 / 3}\left[2^{2 / 3} C_{F} \rho_{\alpha}(\mathbf{r})^{8 / 3}\right.\right. \\
& +2^{2 / 3} C_{F} \rho_{\beta}(\mathbf{r})^{8 / 3}-\rho(\mathbf{r}) t_{w}+\frac{1}{9}\left(\rho_{\alpha}(\mathbf{r}) t_{w}^{\alpha}+\rho_{\beta}(\mathbf{r}) t_{w}^{\beta}\right) \\
& \left.\left.+\frac{1}{18}\left(\rho_{\alpha}(\mathbf{r}) \nabla^{2} \rho_{\alpha}(\mathbf{r})+\rho_{\beta}(\mathbf{r}) \nabla^{2} \rho_{\beta}(\mathbf{r})\right)\right] e^{-c \rho(\mathbf{r})^{-1 / 3}}\right\} d \mathbf{r}
\end{aligned}
$$

where $a=0.04918$ a.u., $b=0.132$ a.u., $c=0.2533$ a.u., $d=0.349$ a.u.,

$$
\begin{gathered}
C_{F}=\frac{3}{10}\left(3 \pi^{2}\right)^{2 / 3} \\
\gamma(\mathbf{r})=2\left[1-\frac{\rho_{\alpha}(\mathbf{r})^{2}+\rho_{\beta}(\mathbf{r})^{2}}{\rho(\mathbf{r})^{2}}\right]
\end{gathered}
$$

and $t_{w}$ is the Weizsacker kinetic energy density.

$$
t_{w}=\frac{1}{8} \frac{|\nabla \rho(\mathbf{r})|^{2}}{\rho(\mathbf{r})}-\frac{1}{8} \nabla^{2} \rho(\mathbf{r})
$$

Becke three-parameter hybrid exchange-correlation functional(B3LYP $)^{4}$

$$
E_{x c}^{\mathrm{B} 3 \mathrm{LYP}}=\left(1-a_{0}-a_{x}\right) E_{x}^{\mathrm{LSDA}}+a_{0} E_{x}^{\text {exact }}+a_{x} E_{x}^{\mathrm{B} 88}+\left(1-a_{c}\right) E_{c}^{\mathrm{VWN}}+a_{c} E_{c}^{\mathrm{LYP}}
$$

where $E_{x}^{\text {exact }}$ is calculated the same way as Hartree-Fock exchange energy, and $E_{c}^{\mathrm{VWN}}$ is an LSDA expression for the correlation energy. ${ }^{5}$ 


\section{Unified density functional(DF07 $)^{6}$}

$$
\begin{gathered}
E_{x c}^{\mathrm{DF} 07}=E_{x}^{\mathrm{exact}}+a_{\mathrm{NDC}}^{\mathrm{opp}} U_{\mathrm{NDC}}^{\mathrm{opp}}+a_{\mathrm{NDC}}^{\mathrm{par}} U_{\mathrm{NDC}}^{\mathrm{par}} \\
+a_{\mathrm{DC}}^{\mathrm{opp}} E_{\mathrm{DC}}^{\mathrm{opp}}+a_{\mathrm{DC}}^{\mathrm{par}} E_{\mathrm{DC}}^{\mathrm{par}}+E_{\text {disp }}+a_{\mathrm{RC}}\left(E_{x}^{B 86}-E_{x}^{B 88}\right)
\end{gathered}
$$

The above energy functional consists of opposite and parallel-spin dynamical correlation energy, $E_{\mathrm{DC}}^{\mathrm{opp}}$ and $E_{\mathrm{DC}}^{\mathrm{par}}$, and non-dynamical correlation potential energy, $U_{\mathrm{NDC}}^{\mathrm{opp}}$ and $U_{\mathrm{NDC}}^{\mathrm{par}}$, components. The other contributions include dispersion interaction energy, $E_{\text {disp, }}$ and a repulsion "fine-tuning" correction, $a_{\mathrm{RC}}\left(E_{x}^{B 86}-E_{x}^{B 88}\right)$. The non-dynamical correlation potential energy for electrons of opposite spin is given by,

$$
U_{\mathrm{NDC}}^{\mathrm{opp}}=\frac{1}{2} \int f \rho_{\alpha}(\mathbf{r}) U_{X \beta}^{\text {exact }} d \mathbf{r}+\frac{1}{2} \int f \rho_{\beta}(\mathbf{r}) U_{X \alpha}^{\text {exact }} d \mathbf{r}
$$

where $f$ is the spin fraction of the effective exchange-correlation hole.

$$
f=\min \left(\frac{1-N_{X \alpha}^{\mathrm{eff}}}{N_{X \beta}^{\mathrm{eff}}}, \frac{1-N_{X \beta}^{\mathrm{eff}}}{N_{X \alpha}^{\mathrm{eff}}}, 1\right)
$$

The exact exchange potential $U_{X \sigma}^{\text {exact }}$ for spin $\sigma$, is given by

$$
U_{X \sigma}^{\text {exact }}(\mathbf{r})=-\frac{1}{\rho_{\sigma}(\mathbf{r})} \sum_{k l} \psi_{k \sigma}(\mathbf{r}) \psi_{l \sigma}(\mathbf{r}) \int \frac{\psi_{k \sigma}\left(\mathbf{r}^{\prime}\right) \psi_{l \sigma}\left(\mathbf{r}^{\prime}\right)}{\left|\mathbf{r}-\mathbf{r}^{\prime}\right|} d \mathbf{r}^{\prime}
$$

The effective local normalization $N_{X \sigma}^{\mathrm{eff}}$ is given by

$$
N_{X \sigma}^{\mathrm{eff}}=\frac{8 \pi A}{a^{3}}
$$

where

$$
\begin{gathered}
a^{2}=\frac{6 Q_{\sigma}}{\rho_{\sigma}(\mathbf{r})} \frac{x}{x-2} \\
A=\rho_{\sigma}(\mathbf{r}) e^{x}
\end{gathered}
$$




$$
\begin{gathered}
\frac{x-2}{x^{2}}\left(e^{x}-1-\frac{x}{2}\right)=-\frac{3}{4 \pi} \frac{Q_{\sigma}}{\left(\rho_{\sigma}(\mathbf{r})\right)^{2}} U_{X \sigma}^{\text {exact }}(\mathbf{r}) \\
Q_{\sigma}=\frac{1}{6}\left(\nabla^{2} \rho_{\sigma}(\mathbf{r})-2 D_{\sigma}\right) \\
D_{\sigma}=\tau_{\sigma}-\frac{\left(\nabla \rho_{\sigma}(\mathbf{r})\right)^{2}}{4 \rho_{\sigma}(\mathbf{r})}
\end{gathered}
$$

and $\tau_{\sigma}$ is the kinetic energy density.

$$
\tau_{\sigma}=\sum_{i}\left(\nabla \psi_{i \sigma}\right)^{2}
$$

The non-dynamical correlation potential energy for electrons of parallel spin is given by,

$$
U_{\mathrm{NDC}}^{\mathrm{par}}=\frac{1}{2} \int \rho_{\alpha}(\mathbf{r}) U_{\mathrm{NDC} \alpha \alpha} d \mathbf{r}+\frac{1}{2} \int \rho_{\beta}(\mathbf{r}) U_{\mathrm{NDC} \beta \beta} d \mathbf{r}
$$

where

$$
U_{\mathrm{NDC} \sigma \sigma}=-A_{\sigma \sigma} M_{\sigma}^{(1)}
$$

The $n^{\text {th }}$ moment of the effective Becke-Roussel(BR) hole is given by,

$$
M_{\sigma}^{(n)}=4 \pi \int_{0}^{\infty} s^{n+2}\left|h_{X \sigma}^{\mathrm{eff}}\right| d s
$$

and

$$
A_{\sigma \sigma}=\min \left(\frac{1-N_{X \sigma}^{\mathrm{eff}}-f N_{X \sigma}^{\mathrm{eff}}}{M_{\sigma}^{(2)}}, \frac{D_{\sigma}}{3 \rho_{\sigma}(\mathrm{r})}\right)
$$

The effective hole is given by,

$$
h_{X \sigma}^{\mathrm{eff}}=-A e^{-a s}
$$

The dynamical correlation energy for electrons of opposite spin and parallel spin are given as,

$$
E_{\mathrm{DC}}^{\mathrm{opp}}=-0.8 \int \rho_{\alpha}(\mathbf{r}) \rho_{\beta}(\mathbf{r}) z_{\alpha \beta}^{2}\left[1-\frac{\ln \left(1+z_{\alpha \beta}\right)}{z_{\alpha \beta}}\right] d \mathbf{r}
$$


and

$$
\begin{gathered}
E_{\mathrm{DC}}^{\mathrm{par}}=\sum_{\sigma} E_{\mathrm{DC}}^{\sigma \sigma} \\
E_{\mathrm{DC}}^{\sigma \sigma}=-0.01 \int \rho_{\sigma}(\mathbf{r}) D_{\sigma} z_{\sigma \sigma}^{4}\left[1-\frac{2}{z_{\sigma \sigma}} \ln \left(1+\frac{z_{\sigma \sigma}}{2}\right)\right] d \mathbf{r}
\end{gathered}
$$

where $z_{\alpha \beta}$ and $z_{\sigma \sigma}$ are correlation lengths.

$$
z_{\sigma \sigma^{\prime}}=c_{\sigma \sigma^{\prime}}\left(\left|U_{X \sigma}^{\mathrm{BR}}\right|^{-1}+\left|U_{X \sigma^{\prime}}^{\mathrm{BR}}\right|^{-1}\right)
$$

where $c_{\alpha \beta}=0.63, c_{\sigma \sigma}=0.88$, and $U_{X \sigma}^{\mathrm{BR}}$ is the Becke-Roussel exchange hole potential. The dispersion energy, $E_{\text {disp }}$ is given as a complex function of atomic multipoles. There are two forms of the DF07 functional, DF07-XX and DF07-BR, depending on which form of the dipole moment of the exchange hole, $d_{X \sigma}(\mathbf{r})$, is used for the dispersion functional. DF07-XX uses a position-dependent dipole moment whereas DF07-BR uses the Becke-Roussel exchange model to approximate $d_{X \sigma}(\mathbf{r})$. The fitted constants of the overall correlation energy expression(Equation A.9) for DF07-XX are $a_{\mathrm{NDC}}^{\mathrm{opp}}=0.511, a_{\mathrm{NDC}}^{\mathrm{par}}=0.615, a_{\mathrm{DC}}^{\mathrm{opp}}=1.073, a_{\mathrm{DC}}^{\mathrm{par}}=0.886$, and $a_{\mathrm{RC}}=0.25$. For DF07-BR the constants are $a_{\mathrm{NDC}}^{\mathrm{opp}}=0.515, a_{\mathrm{NDC}}^{\mathrm{par}}=0.637, a_{\mathrm{DC}}^{\mathrm{opp}}=1.070, a_{\mathrm{DC}}^{\mathrm{par}}=0.985$, and $a_{\mathrm{RC}}=0.27$. 


\section{References}

(1) J. C. Slater. Phys. Rev., 81:385-390, 1951.

(2) A. D. Becke. Phys. Rev. A, 38:3098-3100, 1988.

(3) C. Lee, W. Yang, and R. G. Parr. Phys. Rev. B, 37:785-789, 1988.

(4) A. Becke. J. Chem. Phys., 98:5648-5652, 1993.

(5) S. Vosko, L. Wilk, and M. Nusair. Can. J. Phys., 58:1200-1211, 1980.

(6) A. D. Becke and E. R. Johnson. J. Chem. Phys., 127:124108, 2007. 




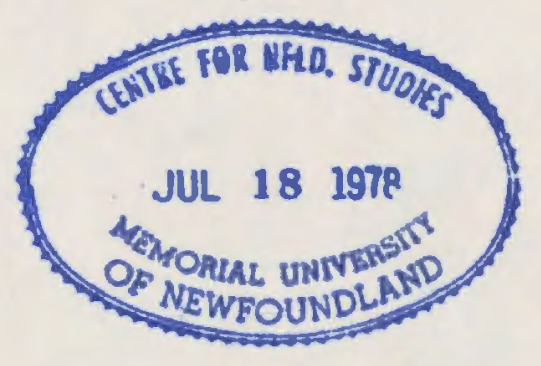


